\title{
Quarterly Report on the Use of the Purge Water Management System at Savannah River Site
}

by

E.. Palmer

Westinghouse Savannah River Company

Savannah River Site

Aiken, South Carolina 29808

C. Beardsley

RECEIVED

FFR 232000

OSTI

DOE Contract No. DE-AC09-96SR18500

This paper was prepared in connection with work done under the above contract number with the U.S.

Department of Energy. By acceptance of this paper, the publisher and/or recipient acknowledges the U.S.

Government's right to retain a nonexclusive, royalty-free license in and to any copyright covering this paper, along, with the right to reproduce and to authorize others to reproduce all or part of the copyrighted paper. 


\section{DISCLAIMER}

This report was prepared as an account of work sponsored by an agency of the United States Government. Neither the United States Government nor any agency thereof, nor any of their employees, makes any warranty, express or implied, or assumes any legal liability or responsibility for the accuracy, completeness, or usefulness of any information, apparatus, product or process disclosed, or represents that its use would not infringe privately owned rights. Reference herein to any specific commercial product, process or service by trade name, trademark, manufacturer, or otherwise does not necessarily constitute or imply its endorsement, recommendation, or favoring by the United States Government or any agency thereof. The views and opinions of authors expressed herein do not necessarily state or reflect those of the United States Government or any agency thereof.

This report has been reproduced directly from the best available copy.

Available for sale to the public, in paper, from: U.S. Department of Commerce, National Technical Information Service, 5285 Port Royal Road, Springfield, VA 22161, phone: (800) 553-6847, fax: (703) 605-6900 email: orders@ntis.fedworld.gov online ordering: http://www.ntis.gov/ordering.htm

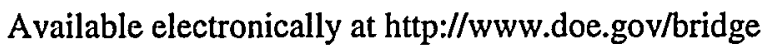

Available for a processing fee to U.S. Department of Energy and its contractors, in paper, from: U.S. Department of Energy, Office of Scientific and Technical Information, P.O. Box 62, Oak Ridge, TN 37831-0062, phone: (865)576-8401, fax: (865)576-5728 email: reportseadonis.osti.gov 


\section{DISCLAIMER}

Portions of this document may be illegible in electronic image products. Images are produced from the best available original document. . 
United States Department of Energy

Savannah River Site

\section{Quarterly Report on the Use of the Purge Water Management System (PWMS) at Savannah River Site (U)}

WSRC-RP-99-4189

Revision 0

November 1999

Westinghouse Savannah River Company LLC

Savannah River Site

Aiken, South Carolina 29808

Prepared for the U.S. Department of Energy under Contract No. DE-AC09-96SR18500

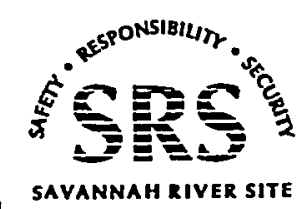




\section{TABLE OF CONTENTS}

LIST OF FIGURES ___ ii

LIST OF TABLES ___ ii

LIST OF ACRONYMS AND ABBREVIATIONS __ iii

EXECUTIVE SUMMARY _

INTRODUCTION__ _ _

METHODOLOGY _ _ 4

RESULTS _ 10

CONCLUSIONS _ _ 24

REFERENCES _ 26

APPÉNDIX A

APPENDIX B 


\section{LIST OF FIGURES}

Figure 1. Schematic drawing of the PWMS 3

Figure 2. Location of wells ABP-1A, ABP-4, and ARP-3 at the Savannah River Site 5

Figure 3. Location of well BGO-33C at the Savannah River Site

Figure 4. Plots of concentrations and activities of analytes collected at well ABP-1A by the PWMS compared to the standard collection program

Figure 5. Plots of concentrations and activities of analytes collected at well ABP -4 by the PWMS compared to the standard collection program

Figure 6. Plots of concentrations and activities of analytes collected at well ARP-3 by the PWMS compared to the standard collection program

Figure 7. Plots of concentrations and activities of analytes collected at well BGO-33C by the PWMS compared to the standard collection program

Figure 8. Plot of tritium activities collected at well BGO-33C by the PWMS compared to the standard collection program

\section{LIST OF TABLES}

Table 1. Schedule of sampling events at PWMS wells

Table 2. Data codes used in the well sample analysis results

- Table 3. PWMS sample results for ABP-1A

Table 4. PWMS sample results for ABP-4

Table 5. PWMS sample results for ARP-3

Table 6. PWMS sample results for BGO-33C

Table 7. Concentrations of copper, lead, and zinc in selected SRS wells 


\section{LIST OF ACRONYMS AND ABBREVIATIONS}

$\begin{array}{ll}{ }^{\circ} \mathrm{C} & \text { degrees Centigrade } \\ \mathrm{gal} & \text { gallons } \\ \mathrm{gpm} & \text { gallons per minute } \\ \mathrm{GSA} & \text { General Separations Area } \\ \mathrm{mg} / \mathrm{L} & \text { milligrams per liter } \\ \mu \mathrm{g} / \mathrm{L} & \text { micrograms per liter } \\ \mathrm{NTU} & \text { nephelometric turbidity units } \\ \mathrm{pCi} / \mathrm{L} & \text { picoCuries per liter } \\ \mathrm{pCi} / \mathrm{mL} & \text { picoCuries per milliliter } \\ \mathrm{PWMS} & \text { Purge Water Management System } \\ \mathrm{SRS} & \text { Savannah River Site }\end{array}$




\section{EXECUTIVE SUMMARY}

The PWMS is a "closed-loop," non-contact system used to collect and return purge water to the originating aquifer after a sampling event without significantly altering the water quality. One of the primary concerns as to its applicability at SRS, and elsewhere, is whether the PWMS might resample groundwater that is returned to the aquifer during the previous sampling event.

The purpose of this quarterly report is to compare groundwater chemical analysis data collected at the four test wells using the PWMS against historical data collected using the standard monitoring well sampling methodology and to determine if the PWMS provides representative monitoring samples.

As part of the demonstration testing of the Purge Water Management System (PWMS) technology at the Savannah River Site (SRS), four wells were equipped with PWMS units in 1997 and a series of sampling events were conducted at each from 1997 to 1998 . Three of the wells (ABP-1A, ABP-4, and ARP-3) are located in A/M Area while the fourth (BGO-33C) is located at the Old Radioactive Waste Burial Ground in the General Separations Area.

Groundwater samples collected by the PWMS in August 1999 continue to be representative of the groundwater quality at monitoring wells $\mathrm{ABP}-1 \mathrm{~A}, \mathrm{ABP}-4, \mathrm{ARP}-3$ and $\mathrm{BGO}-33 \mathrm{C}$. There is a continuing high degree of similarity between analyte concentrations derived from the PWMS samples and those obtained from historical protocol sampling. The latest sample results from sampling with the PWMS extend all previously existing concentration trends to the present time.

Special sampling events conducted in August and October 1999 determined that the source of metals concentrations in PWMS effluent samples do not originate from the PWMS units but, instead, come from (1) elevated turbidity levels resulting from natural aquifer materials entrained in initial purge water from the agitation of the well water during the sampling activity, and (2) specifically for copper, lead, and zinc, the dissolution of the metal components of well appliances and/or preferential partitioning of the metals on colloidal particles that remain suspended in the water standing in the well between samples. 


\section{INTRODUCTION}

Standard procedure for obtaining protocol groundwater monitoring samples at the Savannah River Site (SRS) calls for extracting or "purging" sufficient quantities of groundwater to allow removal of stagnant water from the well and to allow certain key indicator parameters to stạbilize prior to collection of samples. The water extracted from a well prior to sample collection is termed "purge water" and must be managed in an approved fashion if it contains hazardous and/or radiological constituents that exceed specified health-based limits described in the Investigation Derived Waste Management Plan (WSRC 1994). Typical management practices include containerization, transportation, treatment, and disposal at Clean Water Act-permitted facilities.

A technology for handling purge water that eliminates the need to containerize and transport this water to a disposal facility has been developed. This technology, termed the Purge Water Management System (PWMS), is currently in the initial stages of deployment at SRS. The PWMS is a "closed-loop," noncontact system used to collect and return purge water to the originating aquifer after a sampling event without significantly altering the water quality. A schematic drawing of the PWMS is in Figure 1.

For any method used to sample groundwater, sample representativeness is a primary concern. Suitability of the PWMS to collect representative monitoring well samples, while allowing for the extraction and return of large volumes of purge water back to the aquifer, is the chief issue to address for the successful demonstration of the technology.

The purpose of this quarterly report is to present the results of groundwater sampling conducted during August 1999 using PWMS units deployed at four typical SRS wells monitoring groundwater contamination.

This report has been prepared in response to the request from the South Carolina Department of Health and Environmental Control (SCDHEC) in a letter dated August 11, 1999 (Appendix A), and these reports will continue throughout the pilot-testing stage of PWMS deployment. 


\section{Standard PWMS Unit}

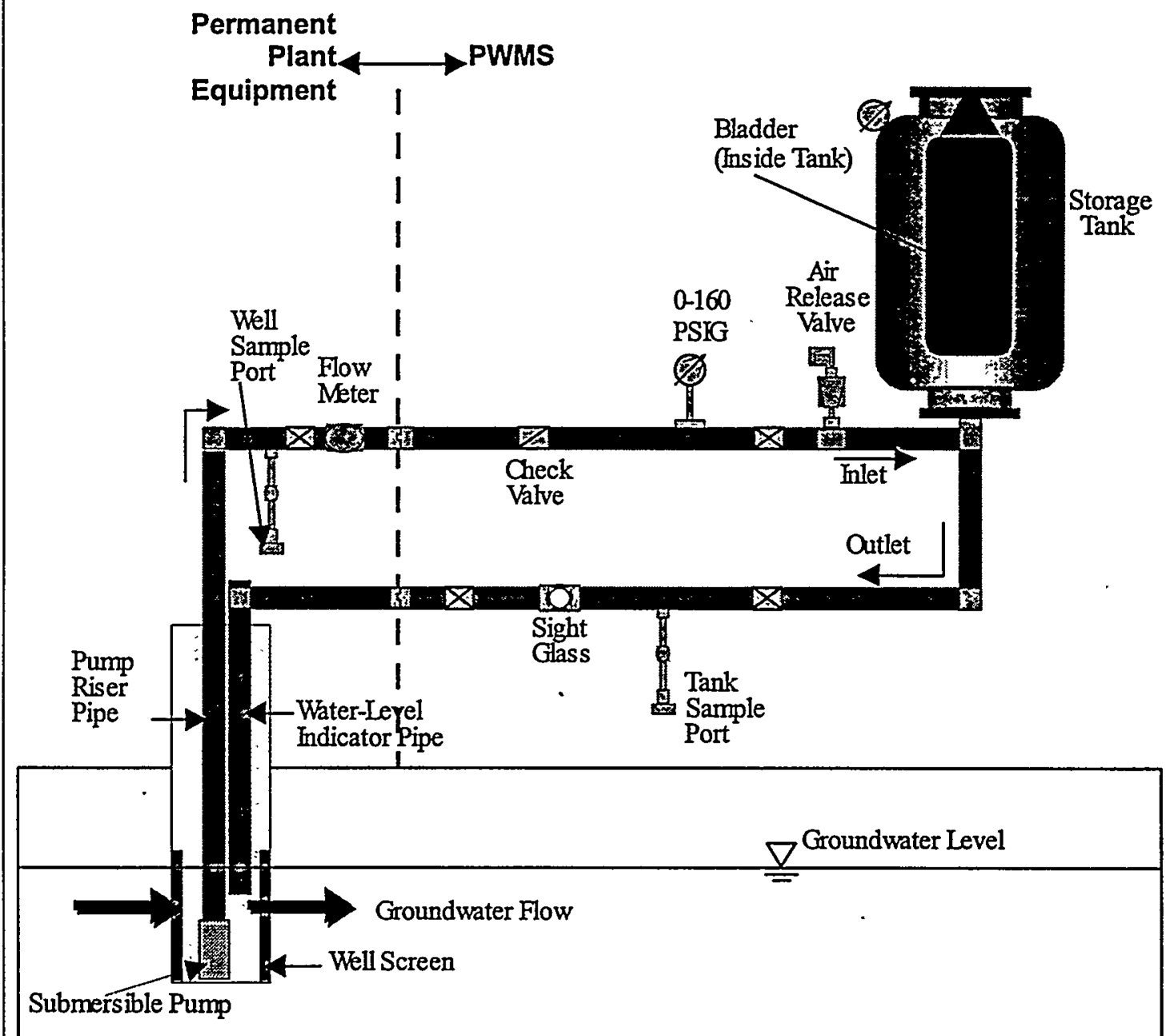

Figure 1. Schematic drawing of the purge water management system 


\section{METHODOLOGY}

\section{PWMS Pilot Well Sampling}

Three wells in the A/M Area and one well in the General Separations Area (GSA) are used to demonstrate effectiveness of the PWMS (Figures 2 and 3):

ABP-1A Located at the A-Area Metals Burning Pit, A/M Area

ABP-4 Located at the A-Area Metals Burning Pit, A/M Area

ARP-3 Located at the A-Area Burning/Rubble Pits, A/M Area

BGO-33C Located at the Old Radioactive Waste Burial Ground, GSA

The sampling schedule followed for evaluation of the use of the PWMS at the four wells is presented in Table 1.

Tạble 1. Schedule of sampling events at PWMS wells

\begin{tabular}{|c|c|}
\hline Well & Sampling Event No.1 \\
\hline ABP-1A & August 17,1999 \\
\hline ABP-4 & August 16,1999 \\
\hline ARP-3 & August 13, 1999 \\
\hline BGO-33C & August 13, 1999 \\
\hline
\end{tabular}

The chemical analysis data from these sample collections were compared with analyte concentration data from protocol samples collected previously at the same wells. The historical data were obtained as part of the routine SRS groundwater-monitoring program. Data for 30 analytes are routinely collected for the comparison of groundwater monitoring results obtained with the PWMS vs. the historical sampling methodology at the four wells (Appendix B). Of these 30 analytes, 15 have been found to effectively represent the trends in groundwater quality at these wells. These 15 analytes include naturally occurring analytes as well as chemical and radiological contaminants known to exist in the vicinity of the wells; the data for these 15 analytes are graphically depicted in this report to compare the PWMS sampling with the historical collections. The 15 analytes, which were also evaluated in detail during previous studies of the PẈMS (Hiergesell et al. 1996; WSRC 1999), include the following:

$\mathrm{pH}$

Specific conductance

Calcium, total recoverable

Potassium, total recoverable

Sodium, total recoverable
Chromium, total recoverable

Iron, total recoverable

Manganese, total recoverable

Aluminum, total recoverable

Lead, total recoverable
Chloride

Carbon tetrachloride

Tetrachloroethylene

Trichloroethylene

Gross alpha 


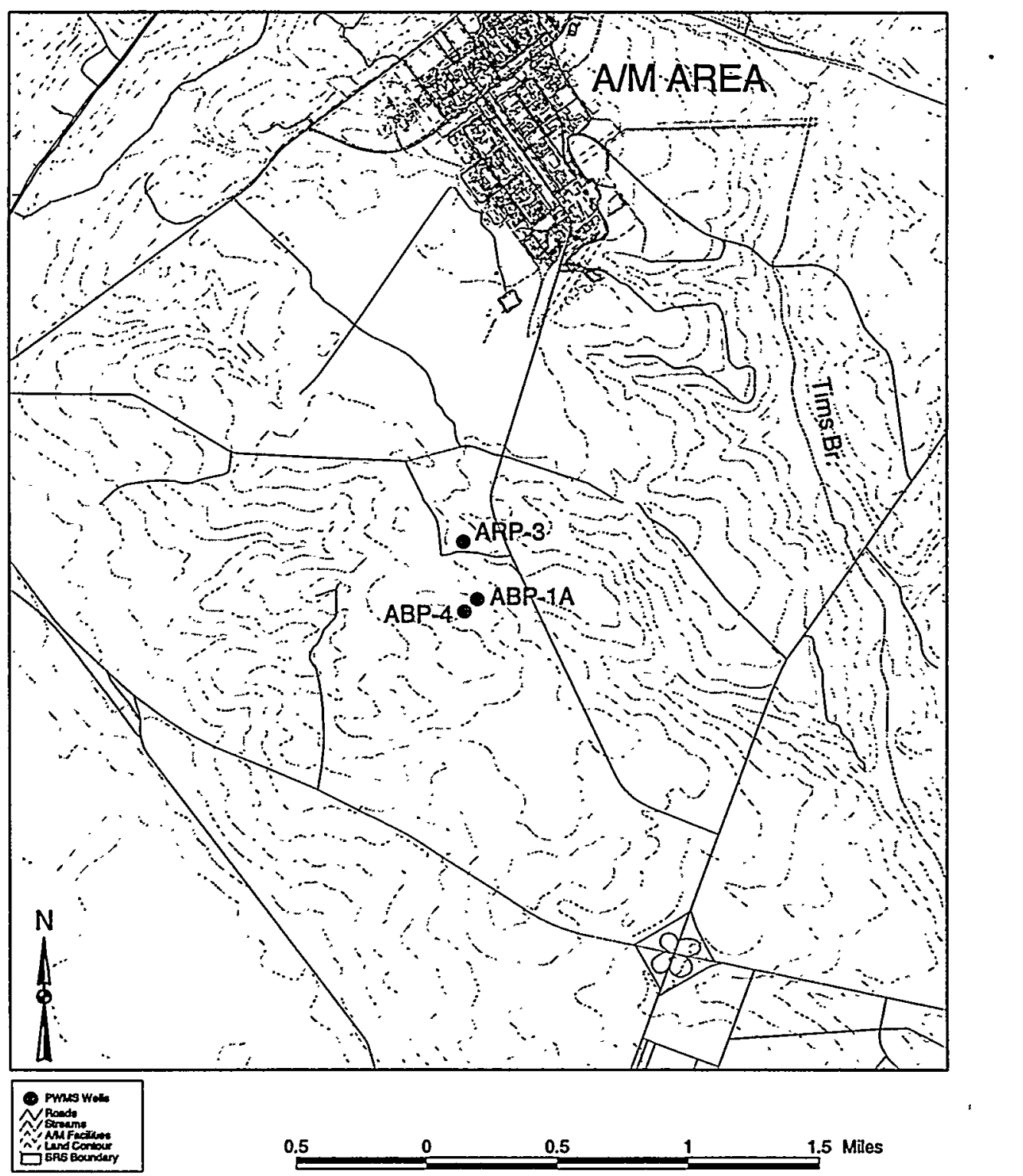

Figure 2. Location of wells ABP-1A, ABP-4, and ARP-3 at the Savannah River Site 


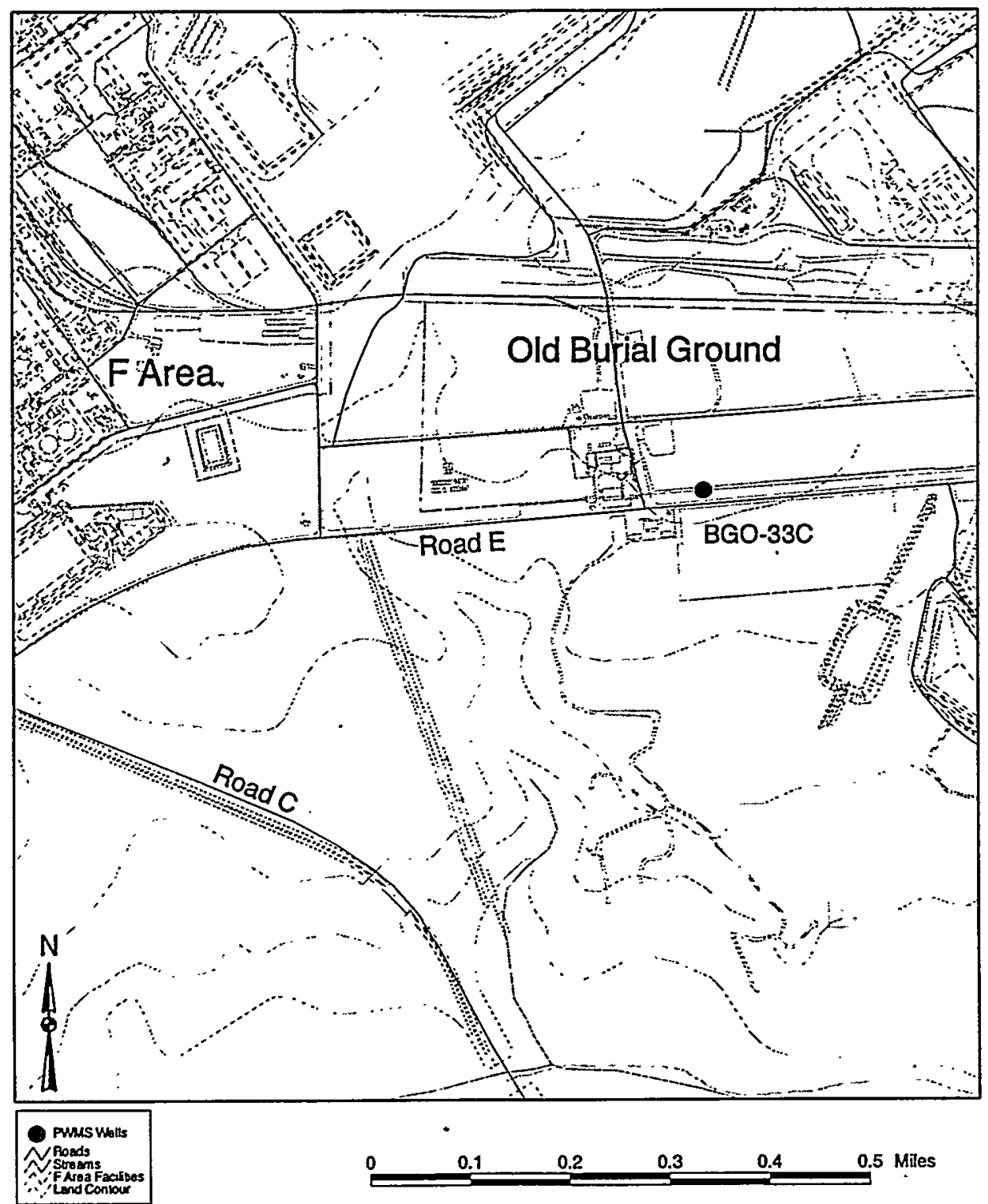

Figure 3. Location of well BGO-33C at the Savannah River Site 
At well BGO-33C, tritium has also been monitored and activities of this radionuclide in the PWMScollected samples and in the historical samples are also compared. Because of a lower hydraulic conductivity at BGO-33C, a tracer study has been previously conducted at that well (WSRC 1999); this study was repeated in August 1999. Potassium bromide (KBr) was injected into the aquifer on August 13, 1999, at BGO-33C. Then, 180 days after the potassium bromide injection, the aquifer will be resampled using the PWMS unit to determine if any of the tracer remains.

Sampling at each of the four wells installed with a PWMS unit was identical and followed SRS standard groundwater sampling procedures (WSRC 1992). Accordingly, groundwater was pumped at a flow rate of approximately one gallon per minute (gpm) into the PWMS unit bladder tank (Figure 1). After a minimum of two well volumes of purge water was pumped into the tank, the field indicator parameters (pH, water temperature, specific conductance, and turbidity) were monitored at the well sampling port to determine the point at which their levels stabilized (i.e., when the measurements for each parameter varied by no more than ten percent of each other). Once stabilization had occurred, a groundwater sample was collected using the PWMS at a location ahead of or "influent" to the PWMS tank while tank filling continued. This sample is, thus, considered to be a "snapshot" of the groundwater unaffected by stagnant well conditions that might have occurred between sampling events.

Samples were shipped to an off-site analytical laboratory under contract to SRS for the performance.of analytical services related to the ongoing groundwater monitoring program. A rigorous laboratory quality assurance process is a major component of this program. Once chemical analysis results were returned from the laboratory, the field and analytical data were validated.

To facilitate the comparison of historical and PWMS sample analyses, the relevant data for each well were downloaded from the SRS Geochemical Information Management System (GIMS) database. Scatter diagrams were constructed for each of the analytes selected to make the comparison for each of the wells. The data from each well were then examined to determine 1) if PWMS samples fall within historical ranges, and 2) if any discernible historical trends could be identified in the historical record and whether PWMS samples reflect a continuation of concentration trends. 


\section{Special Metals Study at PWMS Pilot Wells ABP-1A, ABP-4, ARP-3, and BGO-33C}

A special sampling event was conducted August 13-17, 1999, at PWMS pilot study wells ARP-3, ABP$1 \mathrm{~A}, \mathrm{ABP}-4$, and $\mathrm{BGO}-33 \mathrm{C}$. The event was conducted to collect additional information identified in a meeting between SRS and SCDHEC representatives on August 2, 1999. The purposes of the sampling event were:

- to re-introduce a tracer into the aquifer at BGO $33 \mathrm{C}$ and

- to document that the source of the elevated metals concentrations observed in previous PWMS tank effluent samples did not originate from the PWMS units themselves, but rather from the silt entrained in the turbid purge water prior to sampling.

The special sampling event for each of the wells entailed obtaining the standard influent sample, as acquired in previous PWMS sampling events at these wells. Two additional influent samples, which were obtained prior to the protocol influent sample, augmented the standard samples. These samples were collected when the turbidity levels were high in order to evaluate whether the turbidity in the purge water was the source of the metals in the effluent samples from the tank. In addition, an effluent sample was collected as the tank contents were emptied into the well.

The sample designations used for the special sampling event are as follows:
A First sample collected while turbidity was elevated
B Second sample collected while turbidity was elevated
I Protocol influent sample, collected after the turbidity and other purge parameters had stabilized
E Effluent sample

Special Sampling Event at MSB-11D for Evaluating the Source of Metals in Effluent Samples from the PWMS Pilot Sampling

Two samples were collected on October 11, 1999, from well MSB-11D at SRS. This sampling event was conducted with the approval of SCDHEC after SRS presented the results of the August 13-17 special sampling event during a meeting on September 21, 1999, at the SCDHEC offices in Columbia. 
After reviewing the pilot testing results from the four PWMS wells (BGO-33C, ABP-1A, ABP-4, and ARP-3), SCDHEC expressed concern about the elevated concentrations of copper, lead, and zinc in the effluent being recharged to the aquifer during the standard PWMS sampling procedure. Their concern was that the PWMS units were introducing these metals to the purge water. SRS presented an alternative hypothesis: that the elevated metals concentrations were being introduced by other sources, including the brass valves and flow meters installed at each SRS monitoring well. The SCDHEC staff approved a special sampling event in which a non-PWMS well would be used for sampling. That well would have the following characteristics:

- of similar construction to the four PWMS wells,

- in a similar aquifer, with similar $\mathrm{pH}$ conditions,

- having a brass flow meter assembly,

- having historical concentrations of copper, lead, and zinc for comparisons, and that

- had not been sampled for at least 6 to 12 months.

Well MSB-11D was selected as an appropriate well for this sampling event because it met all of the required characteristics, including the fact that the well had not been sampled since 1995 . The sampling procedure was as follows:

- The well submersible pump was connected to a portable electric generator; the camlock fitting on the end of the discharge was removed; the valve leading to the normal sample port was closed; the valve in front of the flow meter was cracked open; and the sample bottles were prepared with the addition of acid preservative.

- The pump was energized to initiate flow to the surface.

- The first.two gallons of water passing through the flow meter (and exiting through the camlock , fitting) were collected in two one-gallon containers, and the valve was closed.

- The valve leading to the sample port was opened and allowed to flush for about five seconds prior to collecting two additional one-gallon samples.

- The pump was turned off and the water in the valve and flow meter assembly was allowed to drain by gravity.

- After the gallon containers had been shaken to homogenize their contents, two $500 \mathrm{~mL}$ composite samples were created (one for the meter sample and one for the port sample) by pouring $250 \mathrm{~mL}$ from each of the related one-gallon containers into the required $500 \mathrm{~mL}$ sample bottle.

- The samples were shipped to an SC-certified laboratory for analysis. 


\section{RESULTS}

\section{PWMS Pilot Well Sampling}

The results of the scatter diagram comparison of the PWMS sample analyses with the record of historical analyses are presented by well in Figures 4 to 8 . The data used in the comparison for each well are provided in Appendix B.

Data codes used in the figures and in the data presented in Appendix B are identified in Table 2.

Table 2. Data codes used in the well sample analysis results

\begin{tabular}{|c|c|}
\hline \multicolumn{2}{|c|}{ Analyte Codes } \\
\hline Code & Analyte \\
\hline FPH & Field pH \\
\hline COND & Specific conductance \\
\hline CATOT & Calcium, total recoverable \\
\hline KTOT & Potassium, total recoverable \\
\hline NATOT & Sodium, total recoverable \\
\hline CRTOT & Chromium, total recoverable \\
\hline FETOT & Iron, total recoverable \\
\hline MNTOT & Manganese, total recoverable \\
\hline ALTOT & Aluminum, total recoverable \\
\hline PBTOT & Lead, total recoverable \\
\hline CL & Chloride \\
\hline CCL4 & Carbon tetrachloride \\
\hline TCLEE & Tetrachloroethylene \\
\hline TRCLE & Trichloroethylene \\
\hline ALPHAG & Gross alpha \\
\hline TRTIU & Tritium \\
\hline Analytical Result Qualifier Codes \\
\hline Code & $\cdot$ \\
\hline ResQual & Qualification \\
\hline \# & Result qualifier \\
\hline J & Result has no qualifier - interpret as reported \\
\hline U, UI & Result is estimated quantity \\
\hline & Result is less than sample quantitation limit \\
\hline
\end{tabular}



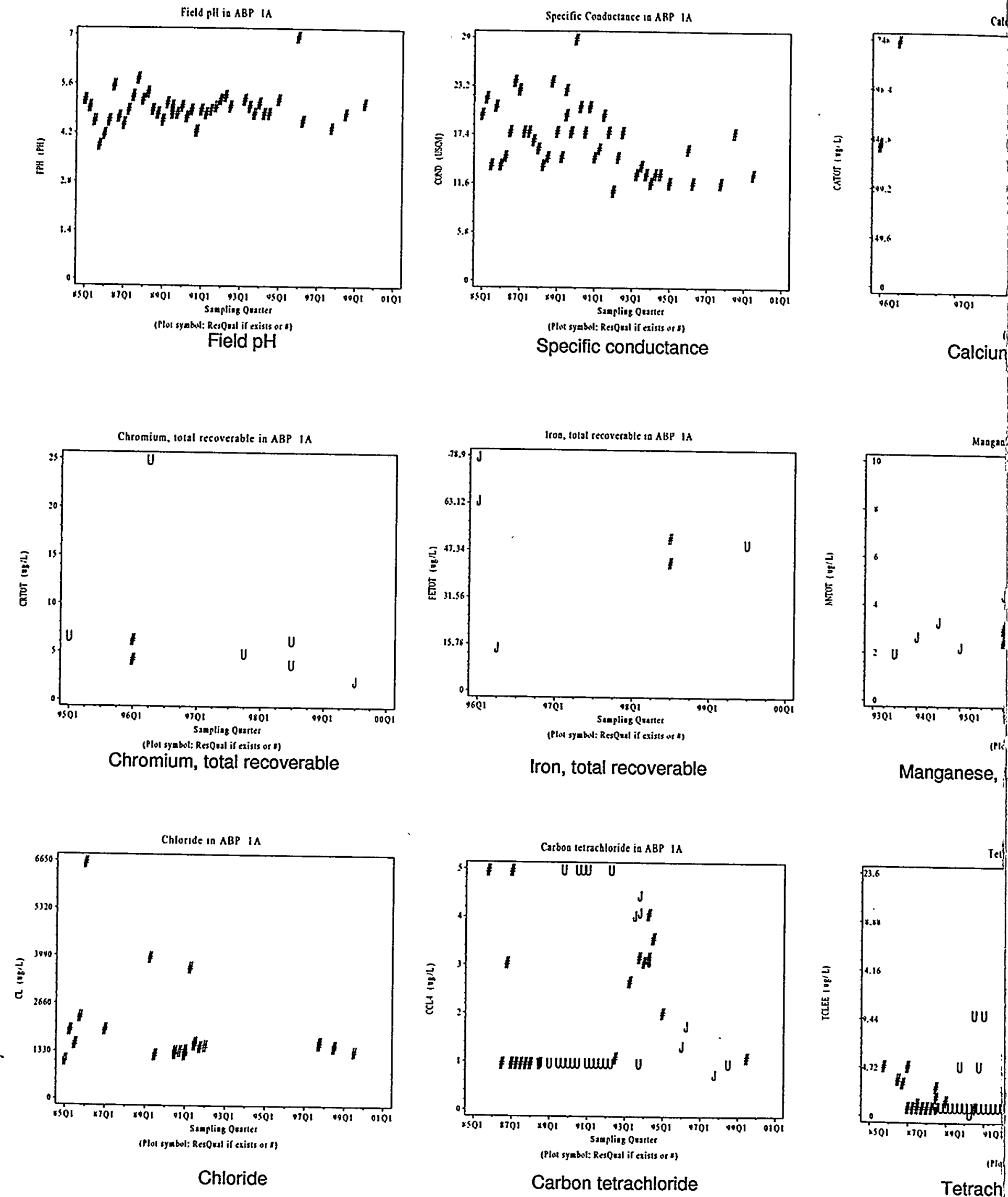

Figure 4. Plots of concentrations and activities of analytes collected at well ABP-1A by the PWMS (in red 

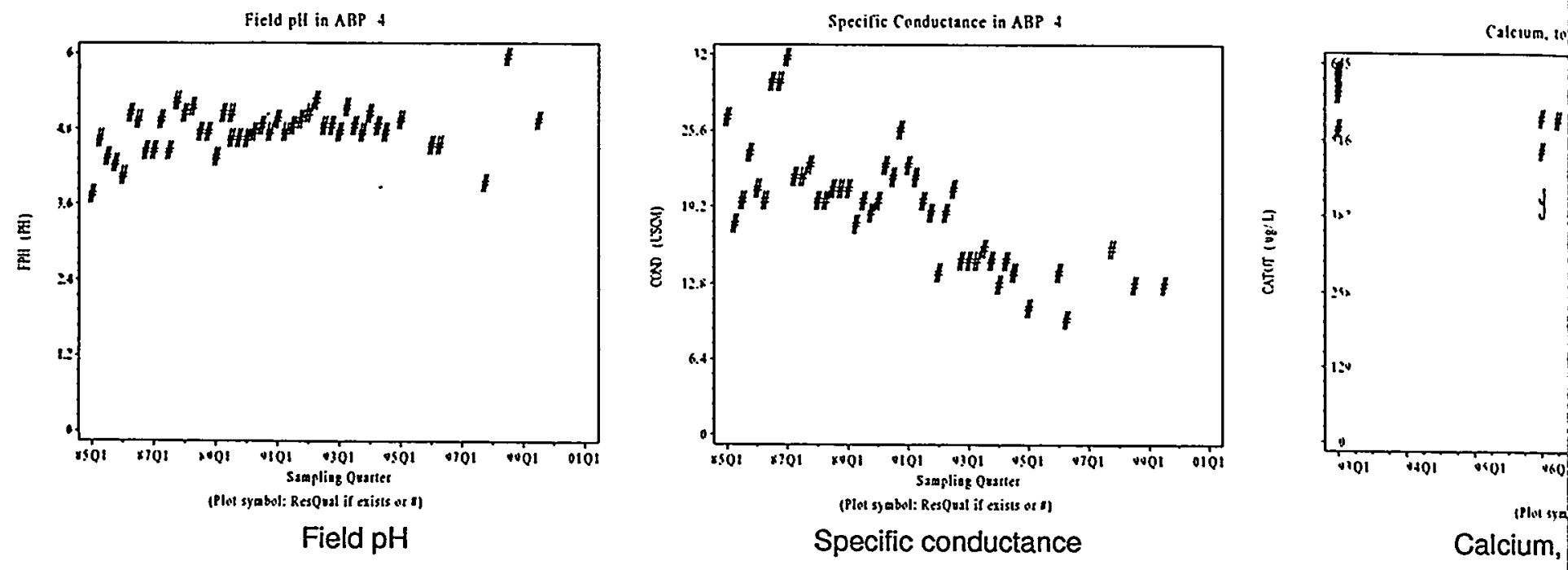

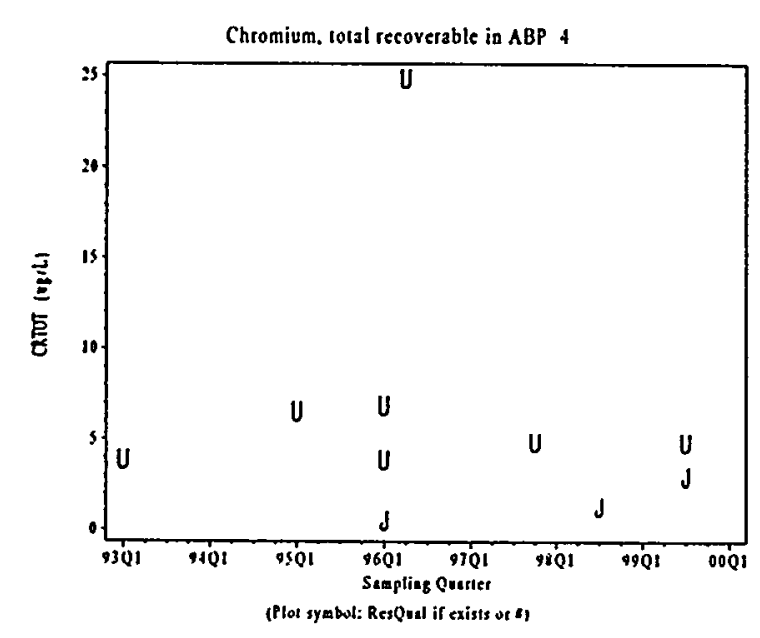

Chromium, total recoverable

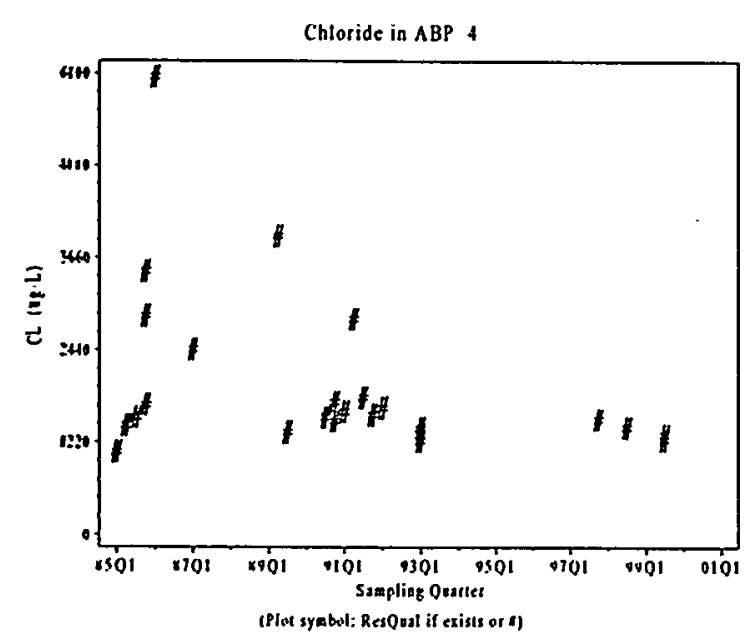

Chloride

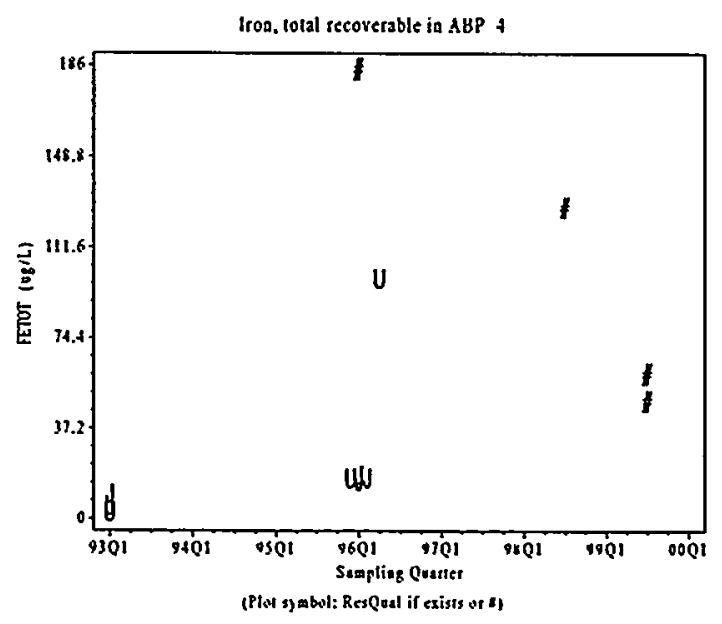

Iron, total recoverable

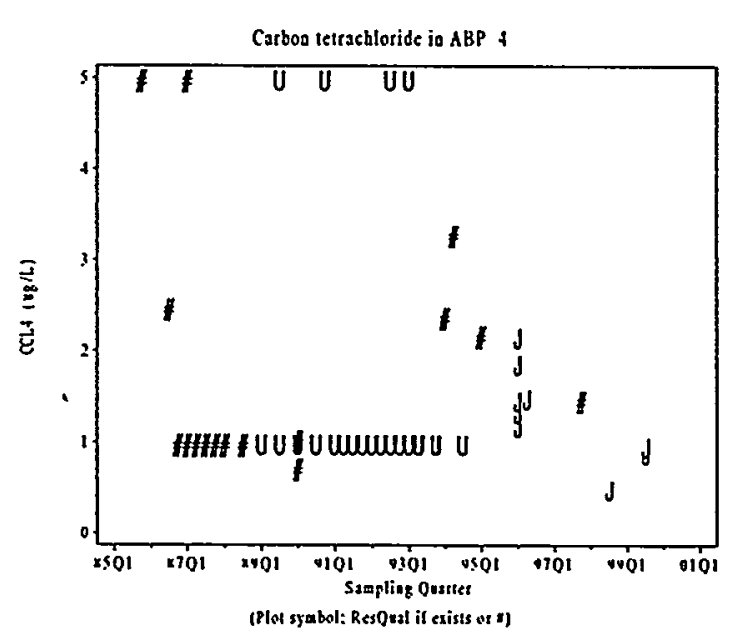

Carbon tetrachloride
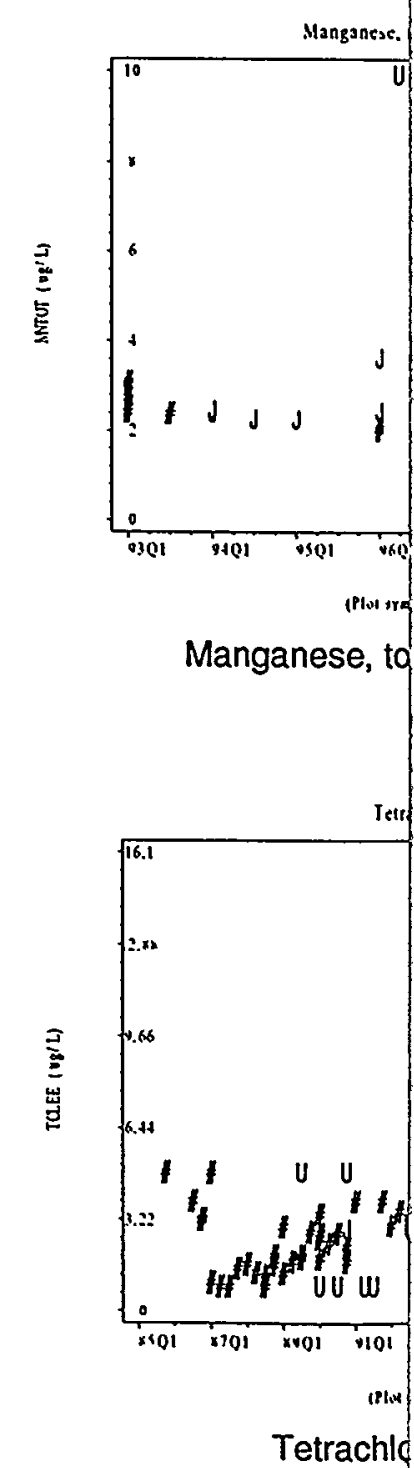

Figure 5. Plots of concentrations and activities of analytes collected at well ABP-4 by the PWMS (in red) 


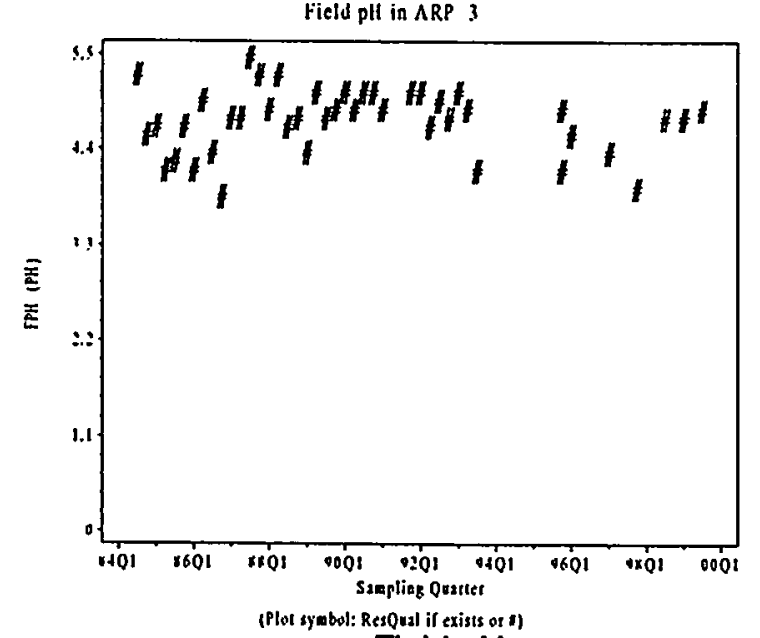

Field pH

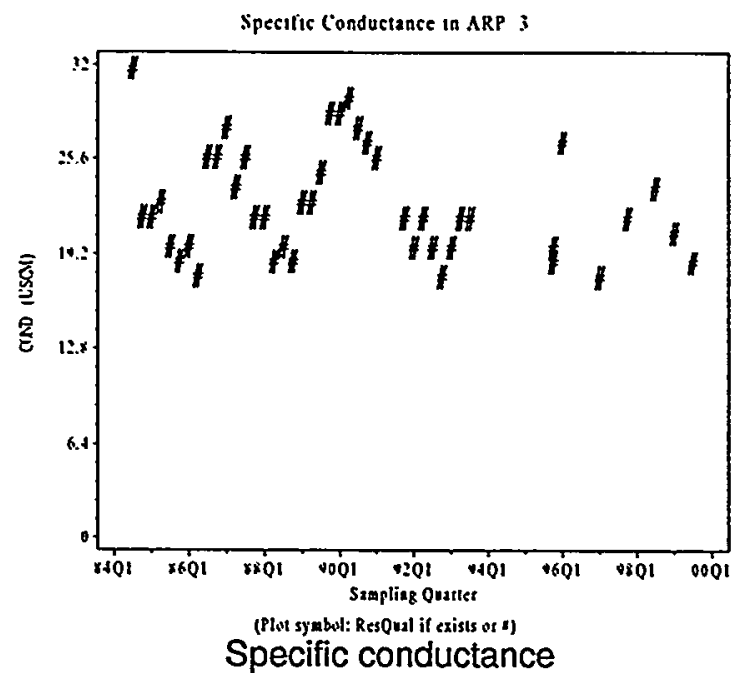

Chromium, lotal recoverable in ARP ;

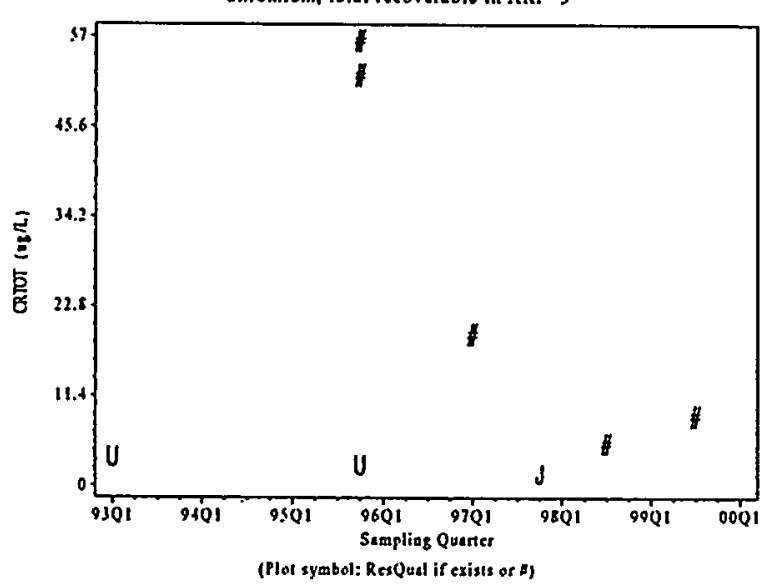

Chromium, total recoverable

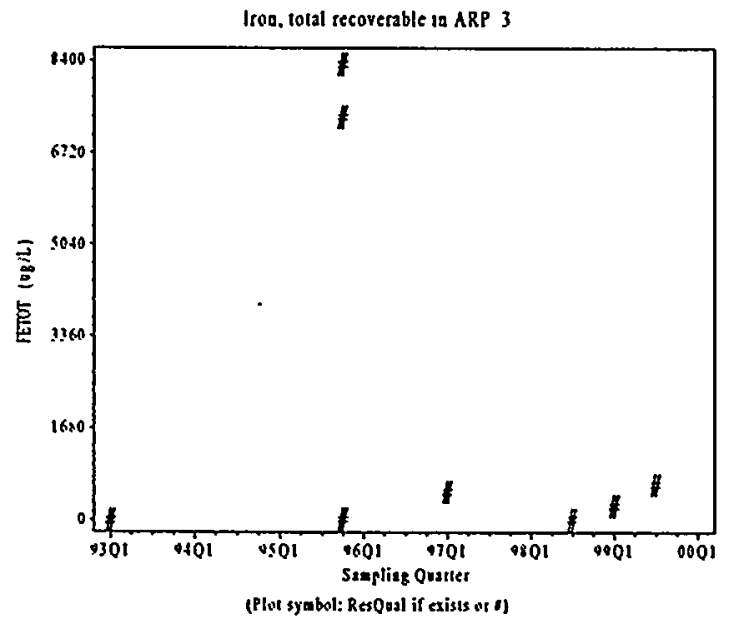

-Iron, total recoverable

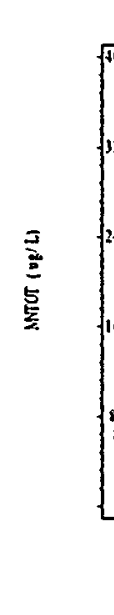

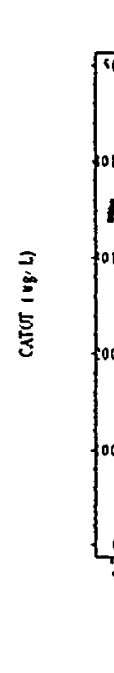

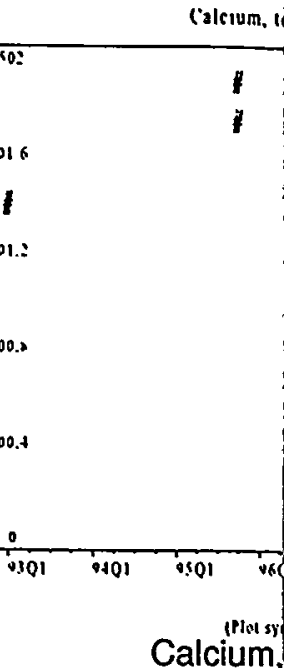

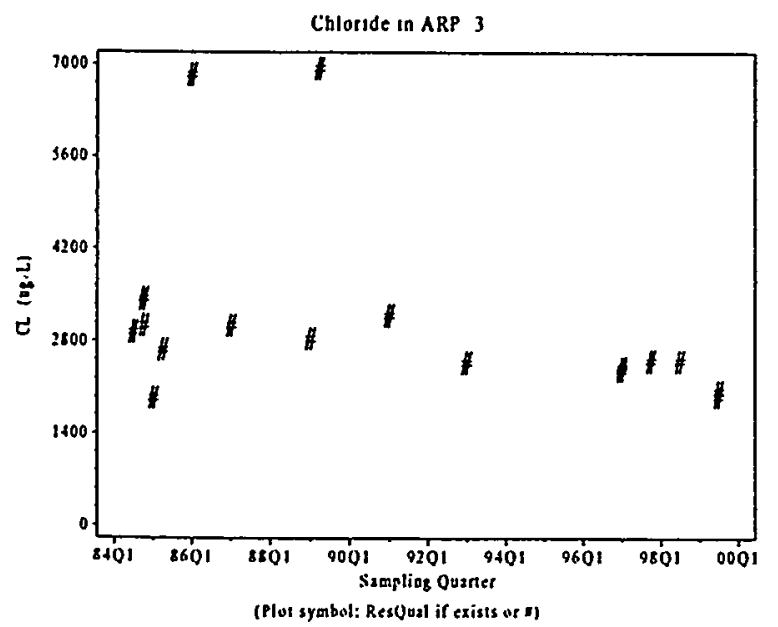

Chloride

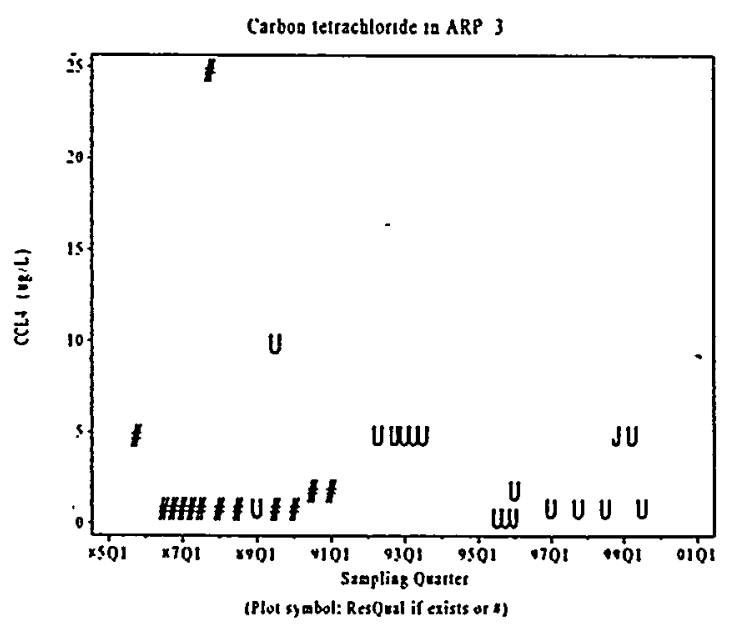

Carbon tetrachloride

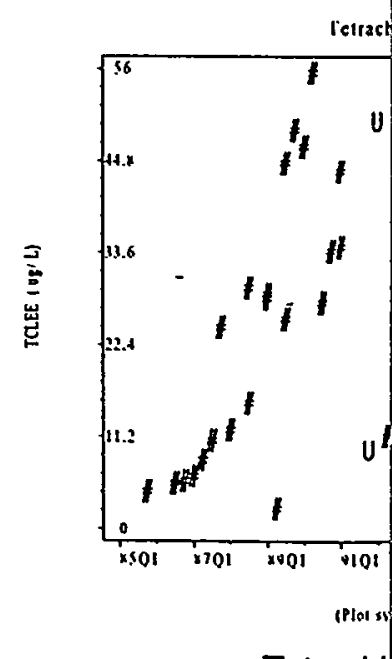

Tetrachl

Figure 6. Plots of concentrations and activities of analytes collected at well ARP-3 by the PWMS (in red) 


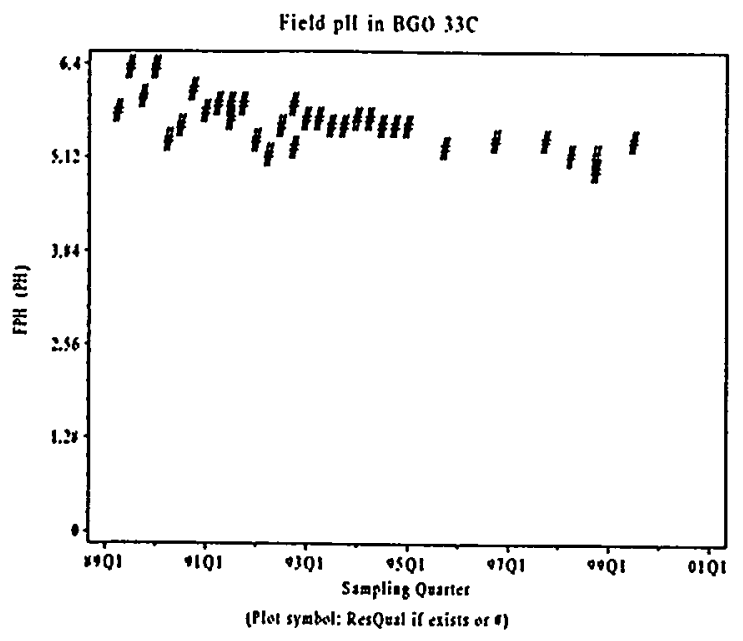

Field $\mathrm{pH}$

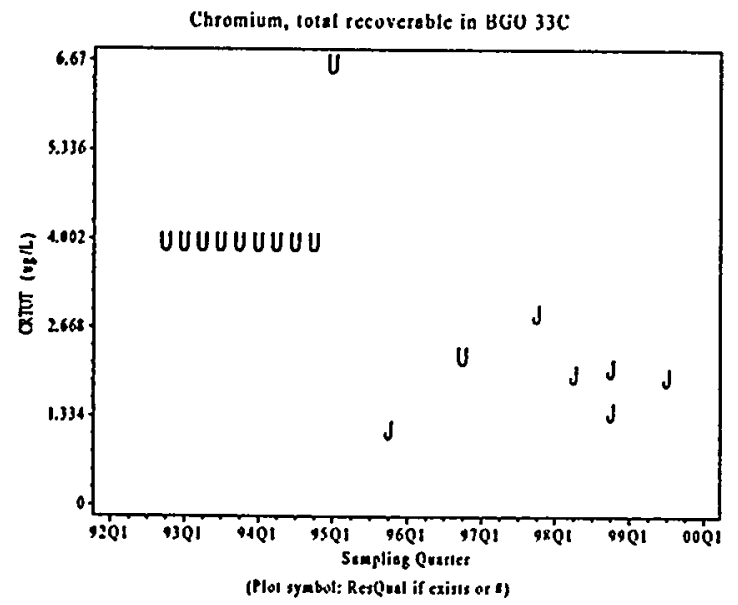

Chromium, total recoverable

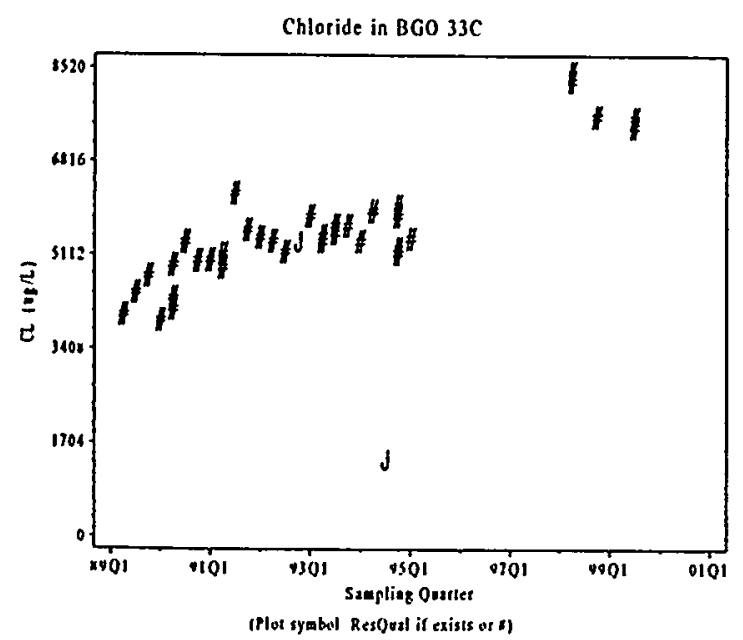

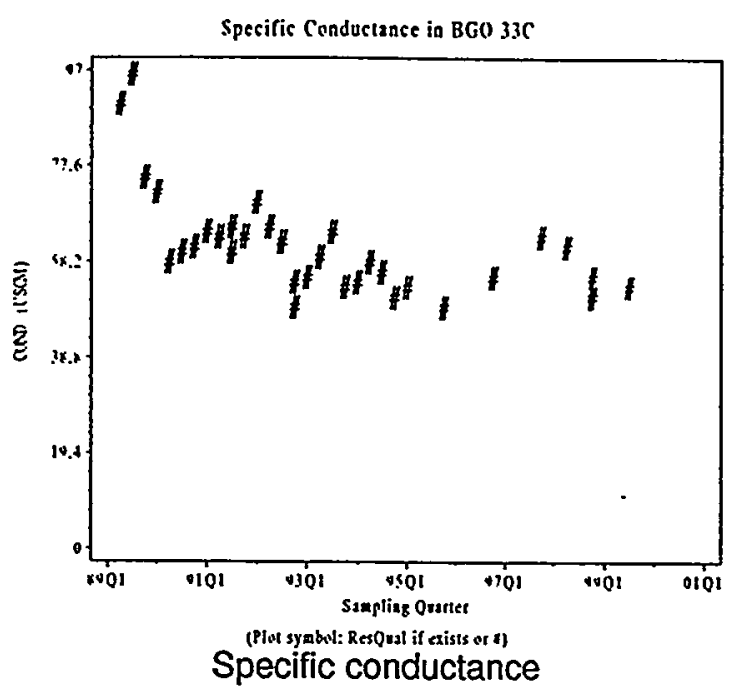

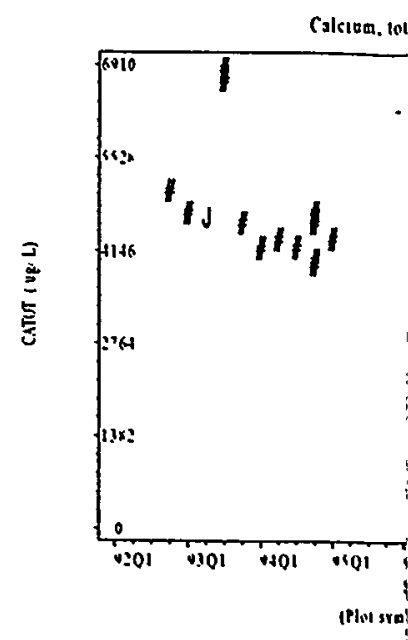

Calcium, $t$
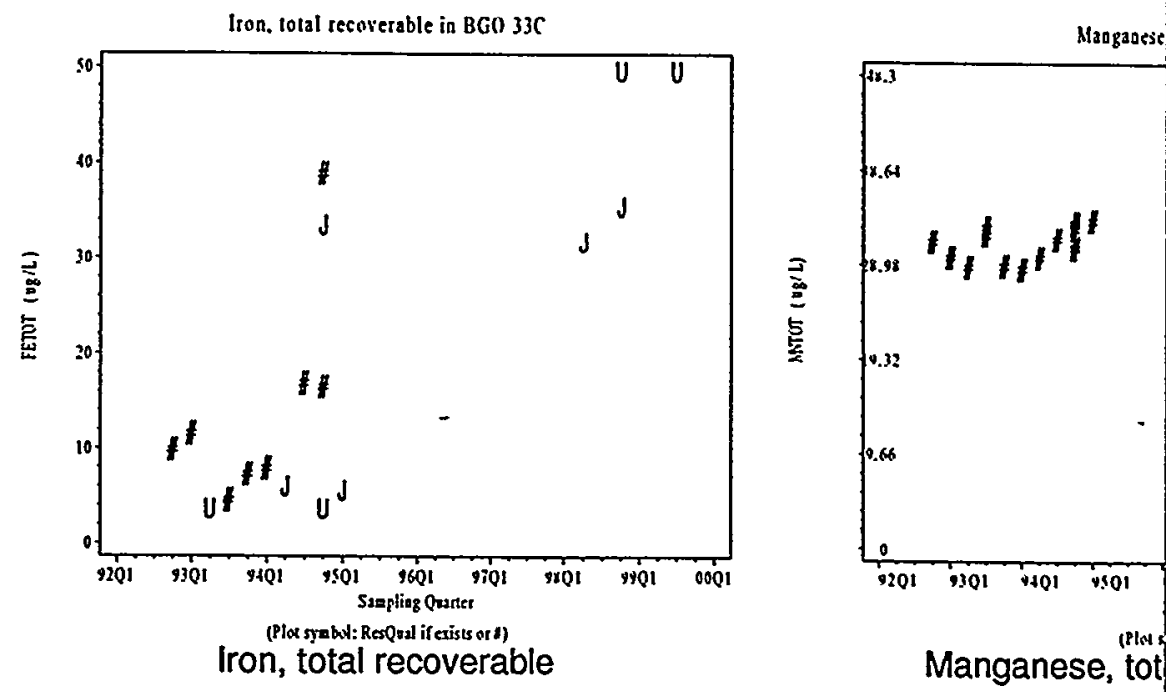

Chloride

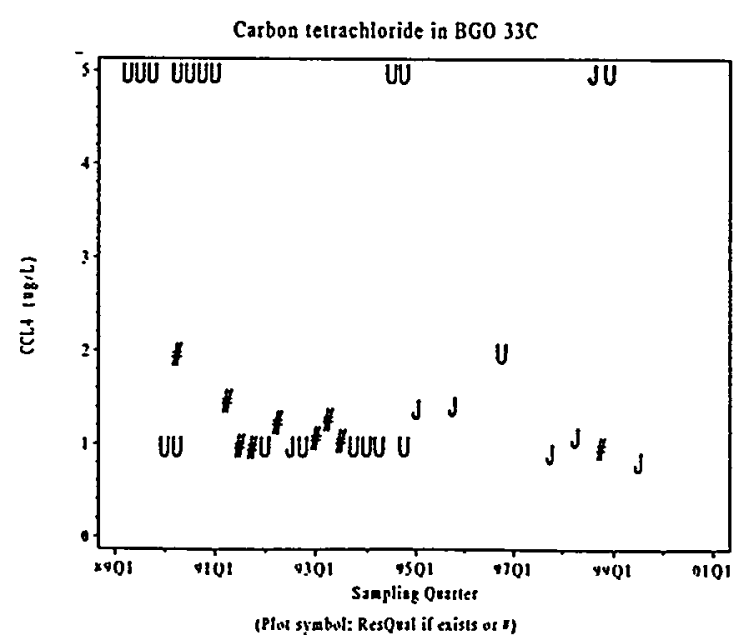

Carbon tetrachloride

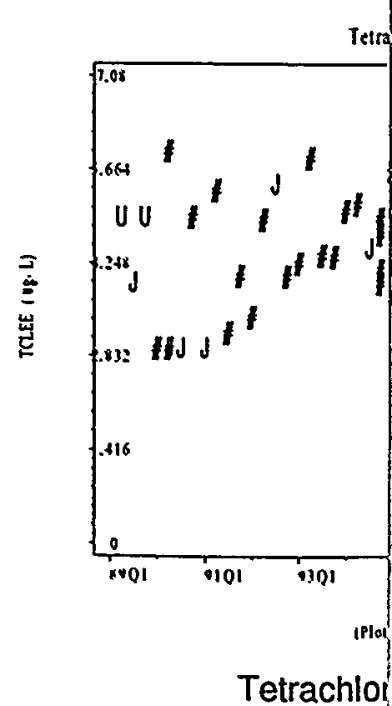

Figure 7. Plots of concentrations and activities of analytes collected at well BGO-33C by the PWMS (in red 


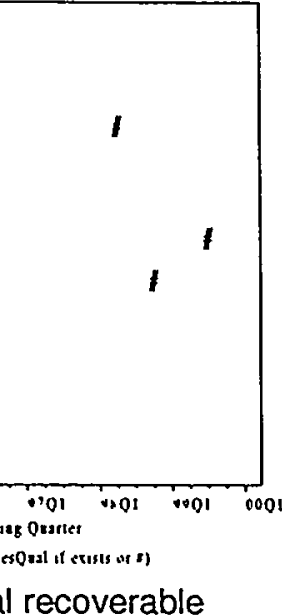

II recoverable it recur erable a $\mathrm{BGO} 33 \mathrm{C}$

1

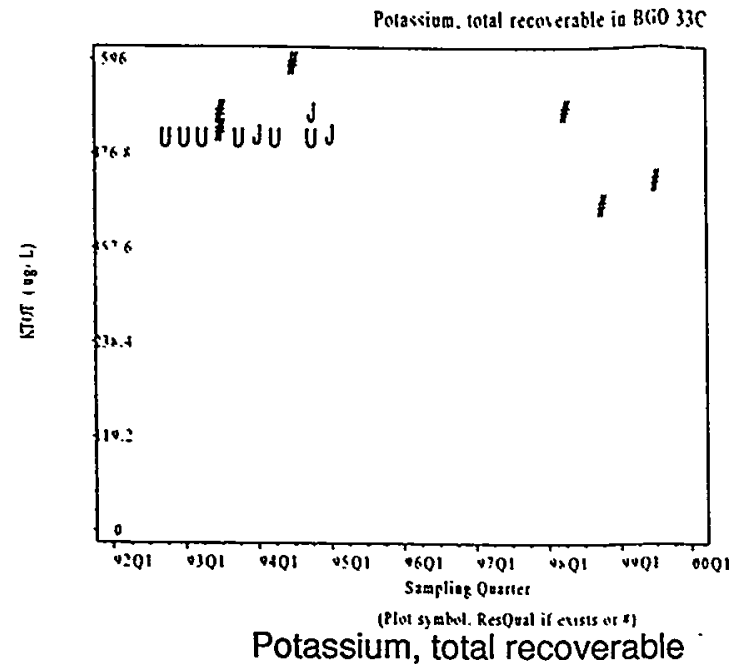

Potassium, total recoverable

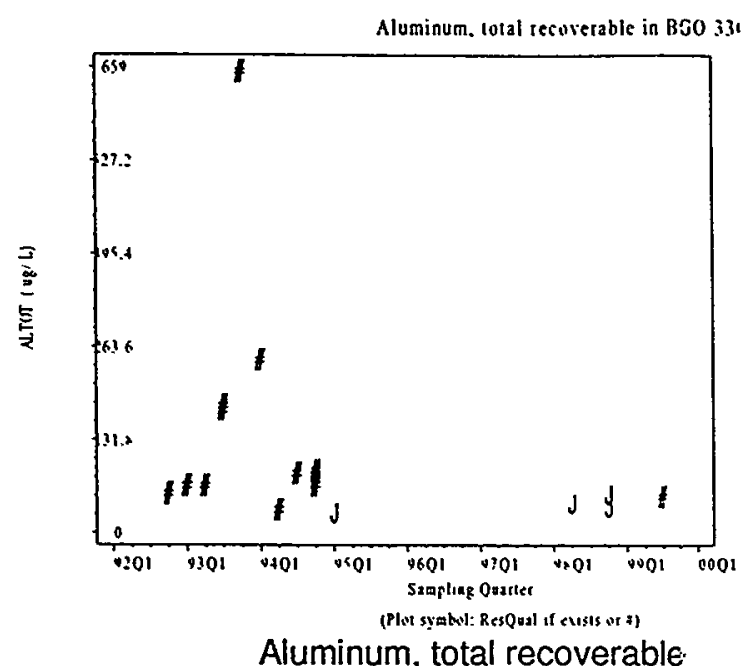

Aluminum, total recoverable

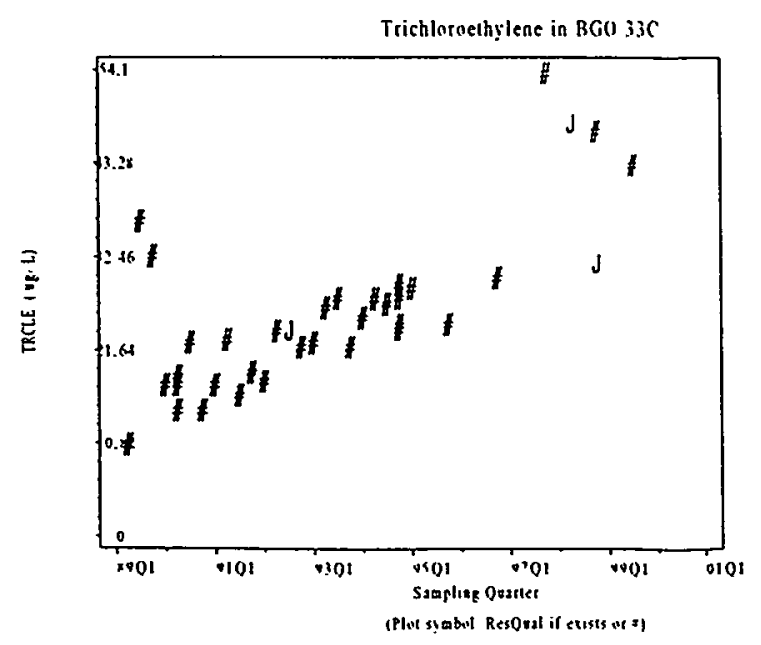

Trichloroethylene

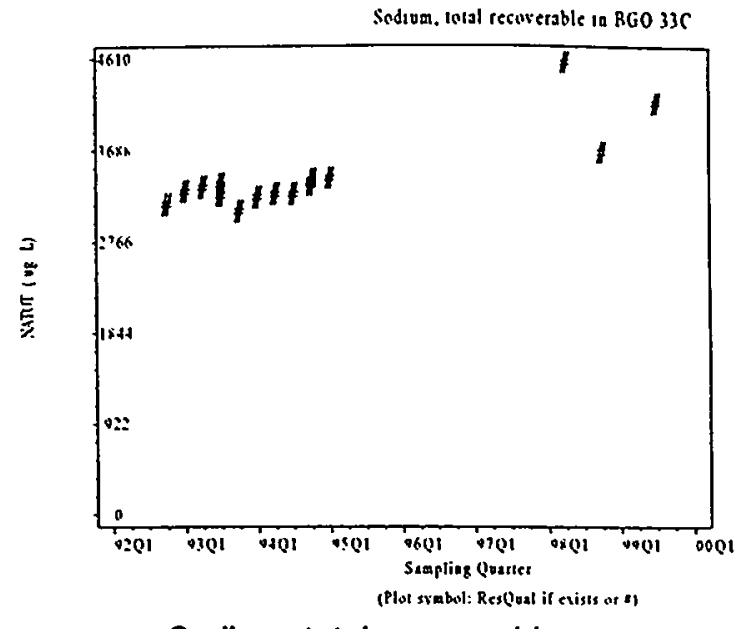

Sodium, total recoverable
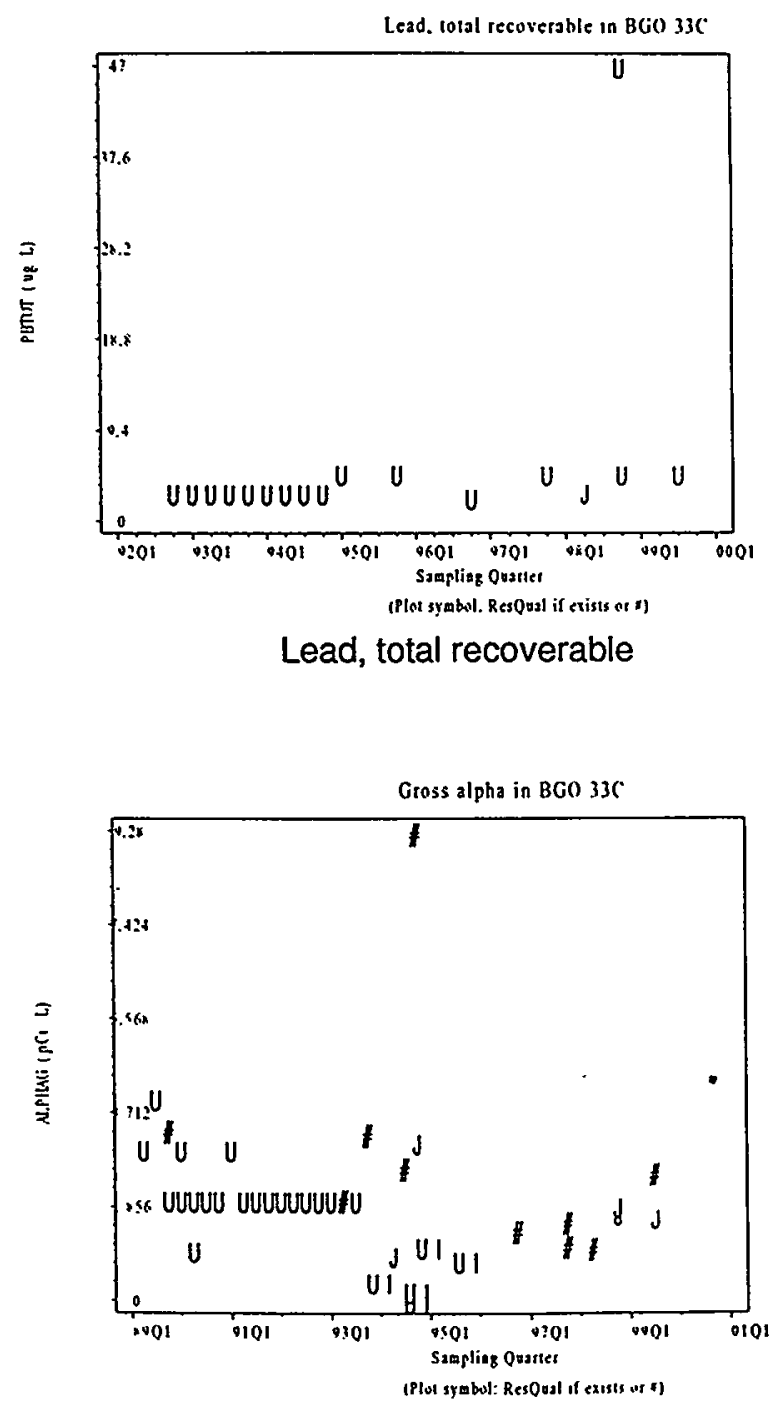

Gross alpha

roethyleae so BGO 330

$$
t^{s}
$$

boll. ResOeal if exisss an

thylene

7


Tritium in BGO 33C

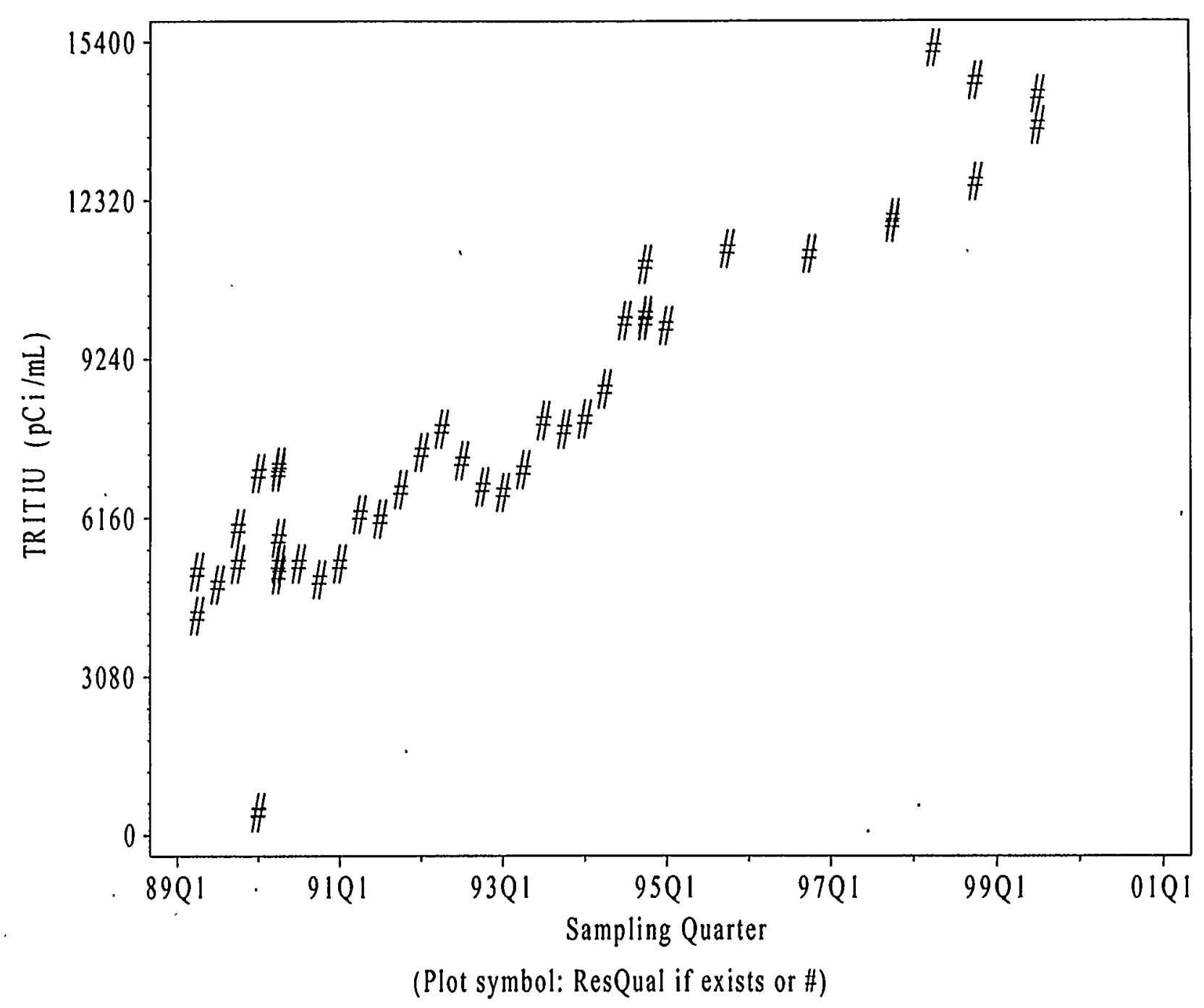

Figure 8. Plot of tritium activities collected at well BGO-33C by the PWMS (in red) compared to the standard collection program (in blue). 


\section{Special Metals Study at PWMS Pilot Wells ABP-1A, ABP-4, ARP-3, and BGO-33C}

The special metals study was designed to test the hypothesis: the source of the elevated metals concentrations in the effluent sample of historical PWMS sampling events was the suspended particles in the purge water, not the PWMS unit itself. Therefore it was expected that the turbidity and metals concentrations would be elevated in the " $\mathrm{A}$ " and "B" samples, depressed in the protocol "I" sample, and somewhat 'averaged' in the "E" sample.

The results described in this analysis are for the metals. Tables 3 through 6 present the concentrations for each constituent (metals, cyanide, and for BGO-33C, radionuclides), in addition to the well purging parameters $(\mathrm{pH}$, specific conductance, turbidity and temperature) measured at the time each sample was collected.

In sampling at all four wells, the field turbidity levels followed the expected profile (high in the " $A$ " and " $B$ " samples, low in the " $I$ " sample and medium in the "E" sample. In regard to specific conductance, three of the wells (ABP-1A, ABP-4, and ARP-3) exhibited stable levels in the "A," "B," and "I" samples while having slightly elevated levels in the " $E$ " sample. BGO-33C was the exception, having its highest specific conductance level in the " $A$ " sample and its second highest level in the " $E$ " sample. In general, the sample analysis results confirm that the samples with the highest turbidity generate the highest concentration of metals. There are a few elements whose highest concentrations consistently do not correspond to the highest turbidity values but appear to correspond with elevated specific conductance values observed in the " $E$ " samples. These patterns are observed at each of the wells as follows:

- In well ABP-1A (Table 3), chromium was the only one of the 13 detected constituents that had its highest concentration observed in the pre-protocol samples. The other 12 detected constituents had their highest concentration observed in the effluent sample, which had the highest specific conductance.

- In well ABP-4 (Table 4), 13 of the 16 constituents detected had their highest concentrations observed in the pre-protocol samples, correlating to elevated turbidities. The other 3 detected constituents (copper, lead, and zinc) had their highest concentrations observed in the effluent sample, which exhibited the highest specific conductance. 
- In well ARP-3 (Table 5), 13 of 18 detected constituents had their highest concentrations observed in very turbid pre-protocol samples. The other 5 detected constituents (copper, lead, magnesium, sodium, and zinc) had their highest concentration in the effluent sample, which had the highest specific conductance.

- In well BGO 33C (Table 6), 11 of 21 detected constituents had their highest concentration observed in pre-protocol samples, correlating to the highest turbidity measurements. The other 10 detected constituents had their highest concentration in the effluent sample, which had the second-highest specific conductance.

The data indicate that the pre-protocol " $A$ " and " $B$ " samples collected at the four wells were obtained when turbidity was still elevated in the purge water. Consequently, the water quality data from those wells can be used to relate the potential for turbidity to be the source of the elevated metals concentrations in the effluent samples.

The following 12 constituents form a group with consistently highest concentrations in the most turbid samples: aluminum, arsenic, barium, beryllium, calcium, iron, manganese, mercury, potassium, selenium, vanadium, and cyanide. This supports the hypothesis that the turbidity is the source of the metals in the effluent samples. The 8 remaining detected constituents generally support the turbidity hypothesis, with the exception of copper, lead, and zinc. The 3 elements of the second group (copper, lead and zinc), whose elevated concentrations do not correlate with elevated turbidity levels, had their highest concentrations in the "E" samples. The occurrence of these elevated concentrations in the "E" samples correlates with the highest specific conductance in three of the four wells tested and with the second highest specific conductance at the fourth well (BGO-33C).

Since turbidity is related to suspended particulate materials, specific conductance is related to dissolved species, and the two groups of metals are correlated to the different field parameters, more than one source may be indicated. The source of the first group is considered to be the turbidity in the sample, with suspended particulate matter being stripped of its metals content when the sample is preserved with nitric acid.

The source of the second group of metals (copper, lead and zinc) is less obvious. At least two potential sources have been considered 1) dissolution of the brass in the well appliances, and/or 2) preferential partitioning of these three metals onto colloidal particles. 
Table 3 PWMS sample results from ABP-1A

\begin{tabular}{|c|c|c|c|c|c|c|}
\hline & & & Sample & & & \\
\hline Analyte Name & $\begin{array}{l}\text { Symbo } \\
1\end{array}$ & $\mathbf{A}$ & $\mathbf{B}$ & $\mathbf{I}$ & $\mathbf{E}$ & Units \\
\hline Field Indicator Parameters: & & & & & & \\
\hline Sample Time & & 7:46 & 8:06 & $8: 38$ & $8: 52$ & \\
\hline Purge Volume & & 9.5 & 25.0 & 91.0 & - & gals \\
\hline $\mathrm{pH}$ & & 4.5 & 4.8 & 5.1 & 5.2 & units \\
\hline Specific Conductance & & 13 & 13 & 13 & 20 & $\mu$ mhos \\
\hline Water temperature & & 20 & 20.9 & 20.3 & 21 & ${ }^{\circ} \mathrm{C}$ \\
\hline Turbidity & & 1.8 & 1.1 & 0.6 & 1.2 & NTU \\
\hline Laboratory Results: & & & & & & \\
\hline Aluminum & ALTOT & $17.8 \mathrm{~J}$ & $50 \mathrm{U}$ & $50 \mathrm{U}$ & $33.6 \mathrm{~J}$ & $\mu \mathrm{g} / \mathrm{L}$ \\
\hline Antimony & SBTOT & $10 \mathrm{U}$ & $10 \mathrm{U}$ & $10 U$ & $10 \mathrm{U}$ & $\mu g / L$ \\
\hline Arsenic & ASTOT & $5 \mathrm{U}$ & $5 \mathrm{U}$ & $5 \mathrm{U}$ & $5 \mathrm{U}$ & $\mu g / L$ \\
\hline Barium & BATOT & $3: 25 \mathrm{~J}$ & $3.05 \mathrm{~J}$ & $2.95 \mathrm{~J}$ & $3.6 \mathrm{~J}$ & $\mu g / L$. \\
\hline Beryllium & BETOT & $5 \mathrm{U}$ & $5 U$ & $5 U$ & $5 \mathrm{U}$ & $\mu \mathrm{g} / \mathrm{L}$ \\
\hline Cadmium & CDTOT & $5 \mathrm{U}$ & $5 \mathrm{U}$ & $5 U$ & $5 \mathrm{U}$ & $\mu \mathrm{g} / \mathrm{L}$ \\
\hline Calcium & СATOT & 525 & 499 & 516 & 574 & $\mu \mathrm{g} / \mathrm{L}$ \\
\hline Chromium & CRTOT & $3.02 \mathrm{~J}$ & 5.84 & $2.25 \mathrm{~J}$ & $2.95 \mathrm{~J}$ & $\mu \mathrm{g} / \mathrm{L}$ \\
\hline Cobalt & COTOT & $5 \mathrm{U}$ & $5 \mathrm{U}$ & $5 \mathrm{U}$ & $5 \mathrm{U}$ & $\mu g / L$ \\
\hline Copper & CUTOT & 5.25 & $3.2 \mathrm{~J}$ & $1.55 \mathrm{~J}$ & 25.2 & $\mu \mathrm{g} / \mathrm{L}$ \\
\hline Iron & FETOT & 99.5 & 97.7 & $50 \mathrm{U}$ & 122 & $\mu \mathrm{g} / \mathrm{L}$ \\
\hline Lead & PBTOT & $5 \mathrm{U}$ & $5 U$ & $5 \mathrm{U}$ & 5.63 & $\mu \mathrm{g} / \mathrm{L}$ \\
\hline Magnesium & MGTOT & 183 & 174 & 177 & 858 & $\mu \mathrm{g} / \mathrm{L}$ \\
\hline Manganese & MNTOT & $10 \mathrm{U}$ & $10 \mathrm{U}$ & $10 \mathrm{U}$ & 12.5 & $\mu \mathrm{g} / \mathrm{L}$ \\
\hline Mercury & HGTOT & $0.2 \mathrm{U}$ & $0.2 \mathrm{U}$ & $0.2 \mathrm{U}$ & $0.2 \mathrm{U}$ & $\mu \mathrm{g} / \mathrm{L}$ \\
\hline Nickel & NITOT & $2.29 \mathrm{~J}$ & $3.41 \mathrm{~J}$ & $1.4 \mathrm{~J}$ & 17.3 & $\mu \mathrm{g} / \mathrm{L}$ \\
\hline Potassium & KTOT & 231 & 218 & 223 & 237 & $\mu \mathrm{g} / \mathrm{L}$ \\
\hline Selenium & SETOT & $5 \mathrm{U}$ & $5 \mathrm{U}$ & $5 \mathrm{U}$ & $5 \mathrm{U}$ & $\mu \mathrm{g} / \mathrm{L}$ \\
\hline Silver & AGTOT & $5 \mathrm{U}$ & $5 \mathrm{U}$ & $5 \mathrm{U}$ & $5 \mathrm{U}$ & $\mu \mathrm{g} / \mathrm{L}$ \\
\hline Sodium & NATOT & 831 & 809 & 821 & 985 & $\mu \mathrm{g} / \mathrm{L}$ \\
\hline Thallium & TLTOT & $5 \mathrm{U}$ & $5 \mathrm{U}$ & $5 \mathrm{U}$ & $5 \mathrm{U}$ & $\mu \mathrm{g} / \mathrm{L}$ \\
\hline Uranium & UTOT & & & $50 \mathrm{U}$ & $50 \mathrm{U}$ & $\mu \mathrm{g} / \mathrm{L}$ \\
\hline Vanadium & VTOT & $5 \mathrm{U}$ & $5 \mathrm{U}$ & $5 \mathrm{U}$ & $5 \mathrm{U}$ & $\mu \mathrm{g} / \mathrm{L}$ \\
\hline Zinc & ZNTOT & $3.6 \mathrm{~J}$ & $4.77 \mathrm{~J}$ & $3.23 \mathrm{~J}^{\circ}$ & 216 & $\mu \mathrm{g} / \mathrm{L}$ \\
\hline Cyanide & $\mathrm{CYN}$ & $10 \mathrm{U}$ & $10 \mathrm{U}$ & $10 \mathrm{U}$ & $10 \mathrm{U}$ & $\mu \mathrm{g} / \mathrm{L}$ \\
\hline
\end{tabular}

$\mathrm{J}=$ Estimated Value below the Quantitation Limit

$\mathrm{U}=$ Not Detected

A $=$ First sample prior to parameter stabilization

$\mathrm{B}=$ Second sample prior to parameter stabilization

I = Protocol Sample

$E=$ Effluent Sample from the Tank 
Table 4 PWMS sample results from ABP-4

\begin{tabular}{|c|c|c|c|c|c|c|}
\hline & & & Sample & & & \\
\hline Analyte Name & $\begin{array}{l}\text { Symbo } \\
1\end{array}$ & $\mathbf{A}$ & $\mathbf{B}$ & $\bar{I}$ & $\mathbf{E}$ & Units \\
\hline Field Indicator Parameters: & & & & & & \\
\hline Sample Time & & $14: 32$ & $14: 40$ & $15: 01$ & $15: 42$ & \\
\hline Purge Volume & & 9.0 & 27.0 & 79.0 & $\cdots$ & gals \\
\hline $\mathrm{pH}$ & & 4.4 & 4.7 & 5.0 & 5.1 & units \\
\hline Specific Conductance & & 13.0 & 13.0 & 13.0 & 14.0 & $\mu$ mhos \\
\hline Water temperature & & 21.0 & 21.1 & 20.5 & 21.3 & ${ }^{\circ} \mathrm{C}$ \\
\hline Turbidity & & 48.9 & 1.4 & 0.5 & 4.9 & NTU \\
\hline Laboratory Results: & & & & & & \\
\hline Aluminum & ALTOT & 3330 & $25.2 \mathrm{~J}$ & $50 \mathrm{U}$ & 57.6 & $\mu g / L$ \\
\hline Antimony & SBTOT & $10 \mathrm{U}$ & $10 \mathrm{U}$ & $10 \mathrm{U}$ & $10 \mathrm{U}$ & $\mu \mathrm{g} / \mathrm{L}$ \\
\hline Arsenic & ASTOT & $5 \mathrm{U}$ & $5 \mathrm{U}$ & $5 \mathrm{U}$ & $5 \mathrm{U}$ & $\mu \mathrm{g} / \mathrm{L}$ \\
\hline Barium & BATOT & 29.7 & $2.48 \mathrm{~J}$ & $2.37 \mathrm{~J}$ & $2.54 \mathrm{~J}$ & $\mu \mathrm{g} / \mathrm{L}$ \\
\hline Beryllium & BETOT & $0.553 \mathrm{~J}$ & $5 \mathrm{U}$ & $5 U$ & $5 \mathrm{U}$ & $\mu \mathrm{g} / \mathrm{L}$ \\
\hline Cadmium & CDTOT & $5 \mathrm{U}$ & $5 \mathrm{U}$ & $5 \mathrm{U}$ & $5 \mathrm{U}$ & $\mu \mathrm{g} / \mathrm{L}$ \\
\hline Calcium & CATOT & 596 & 533 & 470 & 479 & $\mu \mathrm{g} / \mathrm{L}$ \\
\hline Chromium & CRTOT & 151 & $2.79 \mathrm{~J}$ & $3.1 \mathrm{~J}$ & $3.2 \mathrm{~J}$ & $\mu \mathrm{g} / \mathrm{L}$ \\
\hline Cobalt & СОTOT & $3.69 \mathrm{~J}$ & $5 \mathrm{U}$ & $5 \mathrm{U}$ & $5 \mathrm{U}$ & $\mu \mathrm{g} / \mathrm{L}$ \\
\hline Copper & CUTOT & 6.11 & $1.64 \mathrm{~J}$ & $1.96 \mathrm{~J}$ & 20 & $\mu \mathrm{g} / \mathrm{L}$ \\
\hline Iron & FETOT & 14800 & 91.2 & 61.4 & 233 & $\mu g / L$ \\
\hline Lead & PBTOT & 5.88 & $5 \mathrm{U}$ & $5 \mathrm{U}$ & 23.9 & $\mu \mathrm{g} / \mathrm{L}$ \\
\hline Magnesium & MGTOT & 324 & 182 & 177 & 245 & $\mu \mathrm{g} / \mathrm{L}$ \\
\hline Manganese & MNTOT & 43.8 & $10 \mathrm{U}$ & $10 U$ & $10 \mathrm{U}$ & $\mu g / L$ \\
\hline Mercury & HGTOT & $0.2 \mathrm{U}$ & $0.2 \mathrm{U}$ & $0.2 \mathrm{U}$ & $0.2 \mathrm{U}$ & $\mu g / L$ \\
\hline Nickel & NITOT & 74.4 & $1.86 \mathrm{~J}$ & $2.63 \mathrm{~J}$ & $2.66 \mathrm{~J}$ & $\mu g / L$ \\
\hline Potassium & KTOT & 376 & 175 & 168 & 172 & $\mu \mathrm{g} / \mathrm{L}$ \\
\hline Selenium & SETOT & $5 \mathrm{U}$ & $5 \mathrm{U}$ & $5 \mathrm{U}$ & $5 \mathrm{U}$ & $\mu \mathrm{g} / \mathrm{L}$ \\
\hline Silver & AGTOT & $5 \mathrm{U}$ & $5 \mathrm{U}$ & $5 \mathrm{U}$ & $5 \mathrm{U}$ & $\mu \mathrm{g} / \mathrm{L}$ \\
\hline Sodium & NATOT & 974 & 1020 & 960 & 957 & $\mu \mathrm{g} / \mathrm{L}$ \\
\hline Thallium & TLTOT & $5 \mathrm{U}$ & $5 \mathrm{U}$ & $5 \mathrm{U}$ & $5 \mathrm{U}$ & $\mu \mathrm{g} / \mathrm{L}$ \\
\hline Uranium & UTOT & & & $50 \mathrm{U}$ & $50 \mathrm{U}$ & $\mu g / L$ \\
\hline Vanadium & VTOT & 13.1 & $5 \mathrm{U}$ & $5 \mathrm{U}$ & $5 \mathrm{U}$ & $\mu \mathrm{g} / \mathrm{L}$ \\
\hline Zinc & ZNTOT & $21 \mathrm{U}$ & $4.12 \mathrm{U}$ & $4.22 \mathrm{U}$ & 88.6 & $\mu \mathrm{g} / \mathrm{L}$ \\
\hline Cyanide & $\mathrm{CYN}$ & $10 \mathrm{U}$ & $10 \mathrm{U}$ & $10 \mathrm{U}$ & $10 \mathrm{U}$ & $\mu \mathrm{g} / \mathrm{L}$ \\
\hline
\end{tabular}

$$
\begin{aligned}
\mathbf{J} & =\text { Estimated Value below the Quantitation Limit } \\
\mathrm{U} & =\text { Not Detected } \\
\mathrm{A} & =\text { First sample prior to parameter stabilization } \\
\text { B } & =\text { Second sample prior to parameter stabilization } \\
\mathrm{I} & =\text { Protocol Sample } \\
\text { E } & =\text { Effluent Sample from the Tank }
\end{aligned}
$$




\section{Table 5 PWMS sample results from ARP-3}

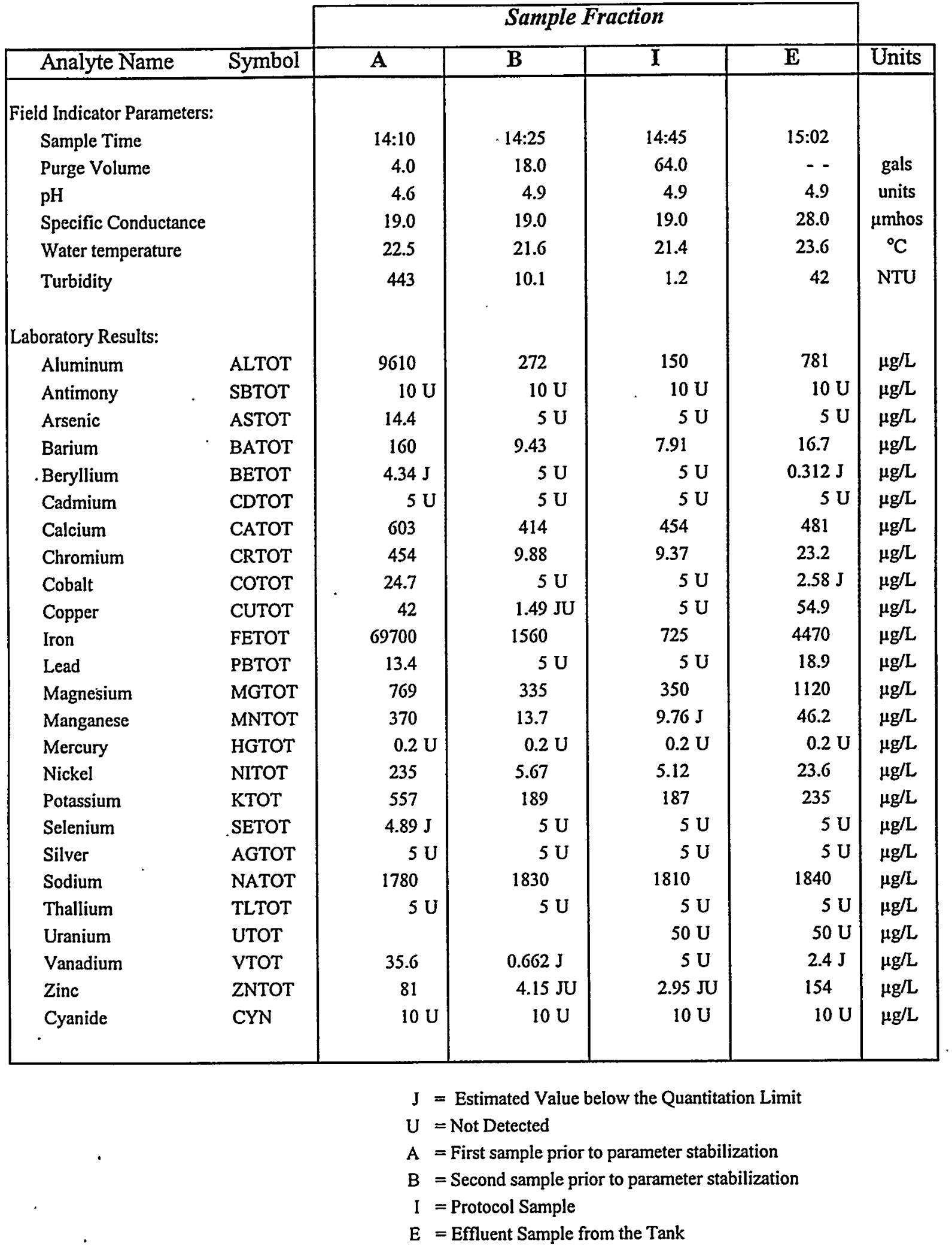


Table 6 PWMS sample results from BGO-33C

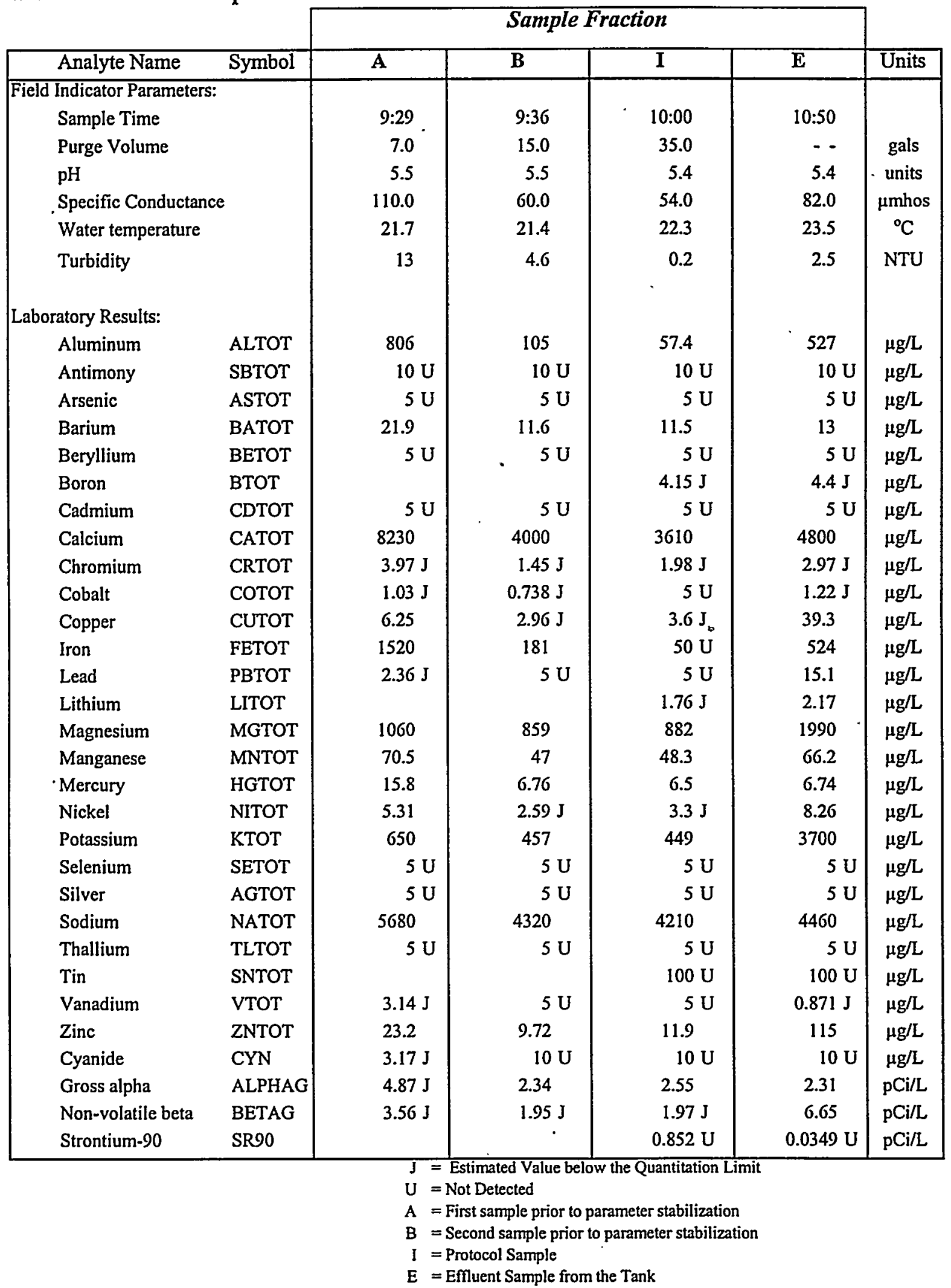




\section{Special Sampling Event at MSB-11D for Evaluating the Source of Metals in Effluent Samples from} the PWMS Pilot Sampling

Table 7 presents the analytical results for copper, lead, and zinc from this sampling event and compares those results to the PWMS pilot test samples and historical results. The table also presents the maximum historical results from the protocol samples at each of the four PWMS pilot wells.

The concentrations in the meter and port samples from MSB-11D exceeded the historical protocol results for the well by factors as large as 71 times, and the port sample results were the highest of the sample results for all 5 wells tested.

With the exception of ARP-3 [where there is a turbidity contrast of over 2 orders of magnitude between the initial (" $\mathrm{A}$ ") and protocol ("I") samples], the initial ("A") sample results are always lower than the historical results. 
Table 7. Concentrations of copper, lead and zinc in selected SRS wells

\begin{tabular}{|c|c|c|c|c|c|c|c|}
\hline $\begin{array}{c}\text { Well ID } \\
\text { and } \\
\text { Sample } \\
\text { Date }\end{array}$ & $\begin{array}{c}\text { Sample } \\
\text { Type }\end{array}$ & $\begin{array}{l}\text { Copper } \\
(\mu \mathrm{g} / \mathrm{L})\end{array}$ & \multicolumn{2}{|c|}{$\begin{array}{c}\text { Lead } \\
(\mu \mathrm{g} / \mathrm{L})\end{array}$} & $\begin{array}{c}\text { Zinc } \\
(\mu \mathrm{g} / \mathrm{L})\end{array}$ & $\begin{array}{c}\text { Purge } \\
\text { Volume } \\
\text { (gallons) }\end{array}$ & $\begin{array}{l}\text { Turbidity } \\
\text { (NTU) }\end{array}$ \\
\hline Octob & Specciăl: & ling Eve & 50 & & $x^{2}+2+2+35$ & $x=\frac{x}{2}=0$ & 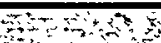 \\
\hline$M S B-11 D$ & historical & $4.64 \mathrm{~J}$ & 3 & $\overline{\mathrm{U}}$ & 39.8 & varied & $\mathrm{NM}$ \\
\hline$(10 / 11 / 99)$ & meter & 44.2 & 49.7 & & 83.6 & 0 & NM \\
\hline & port & 150 & 212 & & 511 & 2 & NM \\
\hline Augus & Speciâl Sa & ṕling Even & 8 & $z^{\prime}$ & 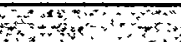 & & 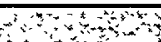 \\
\hline$B G O-33 C$ & historical & 13.9 & 3.07 & & 32.4 & varied & Varied \\
\hline (8/13/99) & A & 6.25 & 2.36 & & 23.2 & 7 & 13 \\
\hline & B & $2.96 \mathrm{~J}$ & $5 \quad$ & $\mathrm{U}$ & 9.72 & 15 & 4.6 \\
\hline & I & $3.6 \mathrm{~J}$ & & $\mathrm{U}$ & 11.9 & 35 & .2 \\
\hline & $\mathrm{E}$ & 39.3 & 15.1 & & 115 & & 2.5 \\
\hline$A R P-3$ & historical & 1.3 & 4.49 & & 7.28 & varied & varied \\
\hline$(8 / 13 / 99)$ & A & 42 & 13.4 & & 81 & 4 & 443 \\
\hline & B & $1.49 \pi$ & & $U$ & $4.15 \mathrm{JU}$ & 18 & 10.1 \\
\hline & I & $5 \quad \mathrm{U}$ & $5 \quad \mathrm{I}$ & $\mathrm{U}$ & $2.95 \mathrm{JU}$ & 64 & 1.2 \\
\hline & E & 54.9 & 18.9 & & 154 & & 42 \\
\hline$A B P-4$ & historical & 19.8 & 14.7 & & 30 & varied & varied \\
\hline$(8 / 16 / 99)$ & A & 6.11 & 5.88 & & $21 \quad U$ & 9 & 48.9 \\
\hline & B & $1.64 \mathrm{~J}$ & & $U$ & $4.12 \mathrm{U}$ & 27 & 1.4 \\
\hline & I & $1.96 \mathrm{~J}$ & 5 & $\mathrm{U}$ & $4.22 \mathrm{U}$ & 79 & .5 \\
\hline & $\mathrm{E}$ & 20 & 23.9 & & 88.6 & & 4.9 \\
\hline$\overline{A B P}-1 A$ & historical & 13.2 & $2.9 \cdot J$ & $J$ & 45.9 & varied & varied \\
\hline$(8 / 17 / 99)$ & A & 5.25 & 5 & $\mathrm{U}$ & 3.6 & 9.5 & 1.8 \\
\hline & B & $3.2 \mathrm{~J}$ & & $\mathbf{U}$ & $4.77 \mathrm{~J}$ & 25 & 1.1 \\
\hline & I & $1.55^{\circ} \mathrm{J}$ & & U & $3.23 \mathrm{~J}$ & 91 & .6 \\
\hline & E & 25.2 & 5.63 & & 216 & & 1.2 \\
\hline
\end{tabular}

Notes:

historical - maximum historical concentration meter - Sample collected from the end of the flow meter port - Sample collected from the standard well sample port $\mathrm{NM}$ - not measured

A - First sample prior to parameter stabilization

B - Second sample prior to parameter stabilization I - Protocol sample after parameter stabilization E - Effluent sample while the tank is being emptied $\mathrm{J}$ - Estimated value below the quantitation limit $\mathrm{U}-$ Not detected, quantitation limit shown 


\section{CONCLUSIONS}

\section{PWMS Pilot Well Sampling}

The PWMS pilot well sampling conducted in August 1999 confirms that the PWMS sampling methodology acquires representative groundwater samples at monitoring wells $A B P-1 A, A B P-4, A R P-3$ and BGO-33C. There was a consistent high degree of similarity between analyte concentrations derived from the PWMS samples and the historical analyte concentrations. Additionally, samples collected using the PWMS methodology extended all previously existing concentration trends to the present time.

\section{Special Metals Study at PWMS Pilot Wells ABP-1A, ABP-4, ARP-3, and BGO-33C}

The data collected in the special metals study indicate that the following 12 constituents form a group that consistently has highest concentrations in the most turbid samples. This supports the hypothesis that turbidity is a major source of the metals in the effluent samples for the following analytes: aluminum, arsenic, barium, beryllium, calcium, iron, manganese, mercury, potassium, selenium, vanadium, and cyanide. The eight remaining detected constituents generally support the turbidity hypothesis, with the exception of copper, lead, and zinc.

The three elements of the second group (copper, lead and zinc), whose elevated concentrations do not correlate with elevated turbidity levels, had their highest concentrations in the samples with the highest specific conductance in three of the four wells tested, and with the second highest conductivity at the fourth well (BGO-33C). At least two potential sources for these three metals have been considered: 1) dissolution of the brass in the well appliances, and/or 2) a preferential partitioning of these three metals onto colloidal particles that remain suspended in the water standing in the well between samples. These very small particles would be the first to enter the pump (and, therefore, the tank) when the well is purged, and would move past the " $\mathrm{A}$ " sample point very quickly.

Three of the second group of metals (copper, lead and zinc) are elements found in well appliance (flow meters, gate valves, pumps, etc.) components that come in contact with the purge water. Potentially, this could be a source of this group of elements, especially if the well water remains in contact with these parts for extended lengths of time, as would occur between sampling events. In the case of wells sampled in this study, contact time may have been as much as a year in three of the wells and eight months in the 
other. Considering that the $\mathrm{pH}$ of groundwater in each of the wells ranges from 4.4 to 5.5 (mild acids), any metallic components could potentially be the source of these elements.

In summary, the source for the majority of the metals is considered to be the turbidity in the water that is generated during the purging process and not the PWMS units. The source of copper, lead, and zinc in the samples required further study; a special sampling event was initiated to that end.

\section{Special Sampling Event at MSB-11D for Evaluating the Source of Metals in Effluent Samples from the PWMS Pilot Sampling}

The sampling results from well MSB 11D document that copper, lead, and zinc concentrations are elevated in the initial purge volumes, even in wells not outfitted with PWMS units. During the well purging process, the concentrations of copper, lead, and zinc eventually decline to lower levels (in many cases to non-detect) prior to collection of the protocol ("I") samples.

Two publications from the USEPA (EPA 1994, EPA 1995) document the existence of lead (and other metals) in groundwater supplies originating from submersible pumps in wells. These studies conclude that older pumps can contribute lead to the groundwater in a well at levels exceeding the action level for drinking water of $15 \mu \mathrm{g} / \mathrm{L}$. The source of the lead is the brass or bronze fittings in the older pumps (made from alloys of copper, lead, and zinc) that leach the lead to the water over time. Their recommended solution was to replace the pump or to purge the water lines prior to using the water if the pump could not be replaced.

These observations support the conclusion that the highest levels of copper, lead, and zinc are contained in the initial purge water coming from the well. The sources of these metals are not in the PWMS units, but elsewhere in the well itself. These sources would include leaching of metals from corrosion of the metallic appliances on the well (the pump, valve, and flow meter, as commonly found elsewhere; e.g., see EPA 1994, 1995), and natural formation materials present in the aquifer. 


\section{REFERENCES}

EPA (U.S. Environmental Protection Agency), 1994, Environmental Fact Sheet: Lead Leaching from Submersible Well Pumps. EPA-747-F-94-001.

EPA (U.S. Environmental Protection Agency), 1995, Fact Sheet: Update on Lead Leaching from Submersible Well Pumps and Private Drinking Water Systems. EPA812-F-95-002.

Hiergesell, R.A., J.E. Cardoso-Neto, and D.W. Williams, 1996. Evaluation of the Purge Water Management System (PWMS) Monitor Well Sampling Technology àt SRS, WSRC-TR-96-0416, Westinghouse Savannah River Company, Aiken, South Carolina.

WSRC, 1992. Hydrogeologic Data Collection, Chapter 15, Sampling Groundwater Monitoring Wells, Procedure Manual WSRC-3Q5, Westinghouse Savannah River Company, Aiken, South Carolina.

WSRC, 1994. Investigation Derived Waste Management Plan, WSRC-RP-94-1227, Revision 2, Westinghouse Savannah River Company, Aiken, South Carolina.

WSRC, 1999. Evaluation of the Validity of Groundwater Samples Obtained Using the Purge Water Mánagement System (PWMS) at the Savannah River Site (U). Westinghouse Savannah River Company, Aiken, South Carolina. 


\section{APPENDIX A}

\section{LETTER FROM SCDHEC}




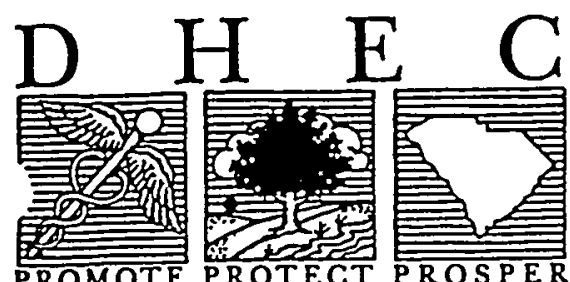

PROMOTE PROTECT PROSPER

2600 Bull Street

Columbia, SC 29201-1708

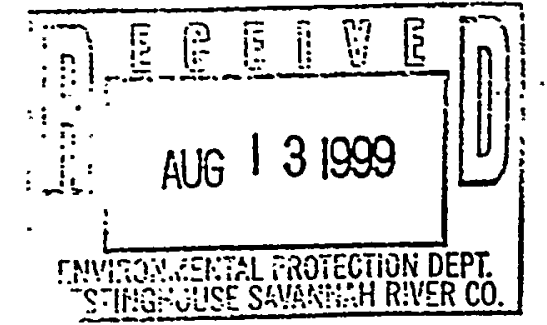

August 11, 1999

\section{Certified Mail}

Ms. Lynn Martin

Facility Support Section

Environmental Protection Department

Savannah River Site

P.O. Box A

Aiken, South Carolina 29808

Re: Savannah River Site

Aiken County

SC1 890008989

Purge Water Management System (PWMS) F/H Proposed Well List (Martin to Devlin), dated August 11, 1999

Dear Ms. Martin,

The Department is in receipt of the referenced letter received via facimilie and submitted by Westinghouse Savannah River Company on behalf of the Savannah River Site. The letter requests approval to install purge water management system (PWMS) units on eight monitoring wells in the F-Area Seepage Basin Hazardous Waste Area and on two monitoring wells in the H-Area Seepage Basin Hazardous Waste Area. These wells include FSB-102C, FSB-104D, FSB-105C, FSB-106C, FSB-107D, FSB-110D, FSB-112D, FSB-117D, HSB-86C, and HSB-86D. The installation of the PWMS units on the proposed monitoring wells is approved. As discussed in the August 2, 1999 meeting with your staff, all wells approved for the PWMS should be redeveloped prior to installation of the unit.

Installation of the PWMS units on the referenced wells is considered an extension of the pilot study that is on-going for this technology. As stated in the referenced letter, SRS will be using a tracer to retest the unit at monitoring wells $B G O-33 \mathrm{C}$ and will be resampling units at wells $A B P-1 A$, ABP-4, and ARP-3. The installation of PWMS units on any additional monitoring wells will be evaluated based on the results of the pilot study, as well as the groundwater sampling results at the $\mathrm{F}$ and $\mathrm{H}$ Area wells. 
SRS must submit quarterly reports on the progress of the pilot study to the Department. The first report will be due to the Department no later than November 15, 1999. Subsequent reports should be submitted two weeks following the end of each quarter. Ms. Marianna Depratter and Ms Jo Cherie Overcash should be copied on these reports.

If you have any questions, please call me at (803) 896-4020.

$$
\begin{aligned}
& \text { Sincerely, } \\
& \text { Cynde L. Devlin, Hydrogeologist } \\
& \text { Hazardous Waste Section } \\
& \text { Division of Hydrogeology } \\
& \text { Bureau of Land and Waste Management }
\end{aligned}
$$

cc: Ms. Crystal Rippy, Permitting

Mr. Robert Baker, DOE

Mr. Tim Mettlen, Lower Savannah EQC

Mr. John Reed, Westinghouse SRS

Ms. Marianna Depratter, Hazardous Waste Section

Ms. Jo Cherie Overcash, Hazardous Waste Section 


\section{APPENDIX B \\ DATA USED IN THE COMPARISON OF THE PWMS GROUNDWATER SAMPLE COLLECTION PROGRAM WITH THE HISTORICAL SAMPLE COLLECTION PROGRAM}


DATA CODES USED IN THE WELL SAMPLE ANALYSIS RESULTS

\begin{tabular}{|l|l|}
\hline \multicolumn{2}{|c|}{ ANALYTE CODES } \\
\hline \multicolumn{1}{|c|}{ Code } & \multicolumn{1}{|c|}{ Analyte } \\
\hline 111TCE & $1,1,1-$ Trichloroethane \\
\hline TCLEA & $1,1,2,2-$ Tetrachloroethane \\
\hline 11DCLE & $1,1-$ Dichloroethane \\
\hline 11DCE & 1.1-Dichloroethylene \\
\hline ALTOT & Aluminum, total recoverable \\
\hline BATOT & Barium, total recoverable \\
\hline BR & Bromide \\
\hline CATOT & Calcium, total recoverable \\
\hline CCLA & Carbon tetrachloride \\
\hline CL & Chloride \\
\hline CRTOT & Chromium, total recoverable \\
\hline COTOT & Cobalt, total recoverable \\
\hline CUTOT & Copper, total recoverable \\
\hline CYN & Cyanide \\
\hline FPH & Field pH \\
\hline F & Fluoride \\
\hline ALPHAG & Gross alpha \\
\hline FETOT & Iron, total recoverable \\
\hline PBTOT & Lead, total recoverable \\
\hline MNTOT & Manganese, total recoverable \\
\hline HGTOT & Mercury, total recoverable \\
\hline NITOT & Nickel, total recoverable \\
\hline NO3NO2 & Nitrate-nitrite as nitrogen \\
\hline BETAG & Nonvolatile beta \\
\hline KTOT & Potassium, total recoverable \\
\hline SETOT & Selenium, total recoverable \\
\hline COND & Specific conductance \\
\hline NATOT & Sodium, total recoverable \\
\hline TCLEE & Tetrachloroethylene \\
\hline T12DCE & Resans-1,2-Dichloroethylene \\
\hline TRCLE & Trichloroethylene \\
\hline ZNTOT & Zinc, total recoverable \\
\hline & QUALIFICATION CODES \\
\hline ResQual & Result qualifier \\
\hline \# & Result has no qualifier - interpret as reported \\
\hline J & \\
\hline U, UI & Restimated quantity \\
\hline
\end{tabular}




\section{WELL ABP-1A DATA}


Field pH in ABP $1 A$

Data Retrieved by AWSA v1 from GIMS on 09NOV99 at 16:22

\begin{tabular}{|c|c|c|c|}
\hline Well & Analyte & Sample_Q Res_Qual Result & Units \\
\hline ABP $1 A$ & FPH & 1985Q1 & 5.2 PH \\
\hline$A B P \quad 1 A$ & $\mathrm{FPH}$ & $1985 Q 2$ & $5 \mathrm{PH}$ \\
\hline ABP $1 A$ & FPH & $1985 Q 3$ & $4.6 \mathrm{PH}$ \\
\hline ABP $1 A$ & FPH & $1985 Q 4$ & $3.9 \mathrm{PH}$ \\
\hline$A B P 1 A$ & FPH & $1986 Q 1$ & $4.2 \mathrm{PH}$ \\
\hline ABP $1 A$ & FPH & $1986 Q 2$ & 4.6 $\mathrm{PH}$ \\
\hline ABP $1 A$ & FPH & $1986 Q 2$ & $4.6 \mathrm{PH}$ \\
\hline$A B P 1 A$ & FPH & $1986 Q 2$ & 4.6 PH \\
\hline ABP $1 A$ & FPH & $1986 Q 3$ & $5.6 \mathrm{PH}$ \\
\hline ABP $1 A$ & FPH & $1986 Q 4$ & 4.7 PH \\
\hline ABP $1 A$ & FPH & 1987Q1 & $4.5 \mathrm{PH}$ \\
\hline ABP $1 A$ & FPH & 1987Q2 & $4.9 \mathrm{PH}$ \\
\hline$A B P 1 A$ & FPH & 1987Q3 & 5.3 PH \\
\hline ABP $1 A$ & FPH & 1987Q4 & $5.8 \mathrm{PH}$ \\
\hline ABP $1 A$ & FPH & 1988Q1 & $5.2 \mathrm{PH}$ \\
\hline ABP 1A & FPH & $1988 Q 1$ & $5.2 \mathrm{PH}$ \\
\hline$A B P 1 A$ & FPH & 1988Q1 & $5.2 \mathrm{PH}$ \\
\hline $\mathrm{ABP} 1 \mathrm{~A}$ & FPH & 198802 & 5.4.PH \\
\hline$A B P 1 A$ & FPH & $1988 Q 2$ & $5.4 \mathrm{PH}$ \\
\hline ABP $1 A$ & FPH & $1988 Q 2$ & $5.4 \mathrm{PH}$ \\
\hline ABP $1 A$ & FPH & $1988 Q 3$ & $4.9 \mathrm{PH}$ \\
\hline ABP $1 A$ & FPH & 1988Q4 & $4.8 \mathrm{PH}$ \\
\hline ABP $1 A$ & FPH & 1989Q1 & 4.6 PH \\
\hline ABP $1 \mathrm{~A}$ & FPH & $1989 Q 1$ & $4.6 \mathrm{PH}$ \\
\hline ABP $1 A$ & FPH & $1989 Q 2$ & $5.1 \mathrm{PH}$ \\
\hline ABP $1 A$ & FPH & $1989 Q 3$ & $4.8 \mathrm{PH}$ \\
\hline ABP $1 \mathrm{~A}$ & FPH & $1989 Q 3$ & $5 \mathrm{PH}$ \\
\hline$A B P 1 A$ & FPH & $1989 Q 4$ & $4.8 \mathrm{PH}$ \\
\hline ABP $1 A$ & FPH & 1989Q4 & $4.8 \mathrm{PH}$ \\
\hline ABP $1 A$ & FPH & 1990Q1 & $5 \mathrm{PH}$ \\
\hline ABP $1 A$ & FPH & 199002 & 4.7 PH \\
\hline$A B P 1 A$ & FPH & 199002 & $4.7 \mathrm{PH}$ \\
\hline ABP $1 A$ & FPH & 1990Q3 & $4.9 \mathrm{PH}$ \\
\hline ABP $1 A$ & FPH & $1990 Q 3$ & $4.9 \mathrm{PH}$ \\
\hline ABP $1 A$ & FPH & $1990 Q 4$ & 4.3 PH \\
\hline$A B P 1 A$ & FPH & 1991Q1 & $4.9 \mathrm{PH}$ \\
\hline ABP $1 \mathrm{~A}$ & FPH & 1991Q1 & $4.9 \mathrm{PH}$ \\
\hline$A B P 1 A$ & FPH & 199102 & $4.8 \mathrm{PH}$ \\
\hline ABP $1 \mathrm{~A}$ & FPH & $1991 Q 3$ & $4.9 \mathrm{PH}$ \\
\hline ABP $1 A$ & FPH & $1991 Q 3$ & $4.9 \mathrm{PH}$ \\
\hline $\mathrm{ABP} 1 \mathrm{~A}$ & FPH & $1991 Q 4$ & $5 \mathrm{PH}$ \\
\hline$A B P 1 A$ & FPH & 1992Q1. & $5.2 \mathrm{PH}$ \\
\hline$A B P 1 A$ & FPH & $1992 Q 1$ & $5.2 \mathrm{PH}$ \\
\hline $\mathrm{ABP} 1 \mathrm{~A}$ & FPH & $1992 Q 2$ & $5.3 \mathrm{PH}$ \\
\hline ABP $1 A$ & FPH & 199202 & $5.3 \mathrm{PH}$ \\
\hline ABP $1 A$ & FPH & 1992Q3 & $5 \mathrm{PH}$ \\
\hline$A B P 1 A$ & FPH & 199302 & $5.2 \mathrm{PH}$ \\
\hline$A B P 1 A$ & FPH & $1993 Q 3$ & $5 \mathrm{PH}$ \\
\hline ABP $1 A$ & FPH & $1993 Q 4$ & $4.8 \mathrm{PH}$ \\
\hline ABP $1 A$ & FPH & 1994Q1 & 5.1 PH \\
\hline$A B P \quad 1 A$ & FPH & $1994 Q 2$ & 4.8 PH \\
\hline$A B P 1 A$ & FPH & $1994 Q 3$ & 4.8 PH \\
\hline$A B P \quad 1 A$ & FPH & 1995Q1 & $5.2 \mathrm{PH}$ \\
\hline$A B P 1 A$ & FPH & $1996 Q 1$ & $7 \mathrm{PH}$ \\
\hline$A B P 1 A$ & FPH & 1996Q2 & 4.6 PH \\
\hline ABP $1 A$ & FPH & $1997 Q 4$ & $4.4 \mathrm{PH}$ \\
\hline ABP $1 A$ & FPH & 199803 & 4.8 PH \\
\hline ABP $1 A$ & FPH & 1999Q3 & 5.1 PH \\
\hline
\end{tabular}

Field $\mathrm{pH}$ in $\mathrm{ABP}$ IA

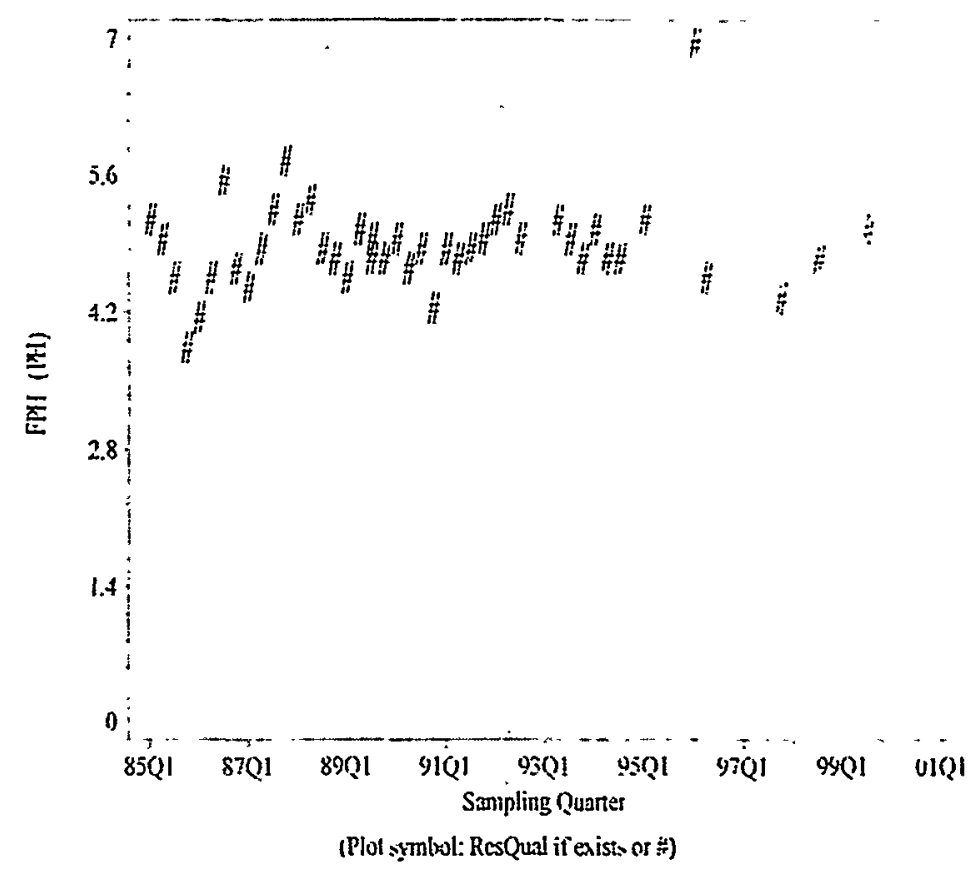


Specific Conductance in ABP $1 A$

Data Retrieved by AWSA v1 from GIMS on 09NOV99 at 16:22

\begin{tabular}{|c|c|c|c|c|}
\hline Well & Analyte & Sample_Q Res_Qual & Result & Units \\
\hline$A B P$ IA & COND & 198501 & & 20 USCM \\
\hline$A B P$ 1A & COND & $1985 Q 2$ & & 22 USCM \\
\hline ABP IA & COND & 1985Q3 & & 14 USCM \\
\hline$A B P$ IA & COND & $1885 Q 4$ & & 21 USCM \\
\hline ABP 1A & COND & 1986Q1 & & 14 USCM \\
\hline$A B P 1 A$ & COND & 198602 & & 15 USCM \\
\hline ABP $1 A$ & COND & 1986Q2 & & 15 USCM \\
\hline$A B P$ IA & COND & 1986Q2 & & 15 USCM \\
\hline ABP 1A & COND & 1986Q3 & & 18 USCM \\
\hline$A B P$ 1A & COND & 198604 & & 24 USCM \\
\hline ABP $1 A$ & COND & 1987Q1 & & 23 USCM \\
\hline ABP $1 A$ & COND & 198702 & & 18 USCM \\
\hline ABP $1 A$ & COND & 1987Q3 & & 18 USCM \\
\hline$A B P 1 A$ & COND & 198704 & & 17 USCM \\
\hline$A B P 1 A$ & COND & 1988Q1 & & 16 USCM \\
\hline$A B P 1 A$ & COND & 1988Q1 & & 16 USCM \\
\hline ABP $1 A$ & COND & 1988Q1 & & 16 USCM \\
\hline ABP $1 A$ & COND & 1988Q2 & & $14 \mathrm{USCM}$ \\
\hline$A B P$ 1A & COND & 1988Q2 & & 14 USCM \\
\hline$A B P \quad 1 A$ & COND & 198802 & & 14 USCM \\
\hline$A B P$ IA & COND & $1988 Q 3$ & & 15 USCM \\
\hline ABP $1 A$ & COND & 1988Q4 & & 24 USCM \\
\hline ABP $1 A$ & COND & 1988Q1 & & 18 USCM \\
\hline$A B P \quad 1 A$ & COND & $1989 Q 1$ & & $18 \mathrm{USCM}$ \\
\hline$A B P$ IA & COND & $1989 Q 2$ & & 15 USCM \\
\hline$A B P \quad 1 A$ & COND & 1989Q3 & & 20 USCM \\
\hline$A B P 1 A$ & COND & 1989Q3 & & 23 USCM \\
\hline$A B P$ 1A & COND & 198904 & & 18 USCM \\
\hline$A B P$ IA & COND & 1989Q4 & & 18 USCM \\
\hline$A B P \backslash A$ & COND & $1890 Q 1$ & & 29 USCM \\
\hline ABP $1 A$ & COND & $1990 \mathrm{Q} 2$ & & 21 USCM \\
\hline$A B P$ 1A & COND & - 199002 & & 21 USCM \\
\hline$A B P$ IA & COND & 1990Q3 & & 18 USCM \\
\hline$A B P 1 A$ & COND & $1990 Q 3$ & & 18 USCM \\
\hline$A B P$ IA & COND & 199004 & & 21 USCM \\
\hline ABP $1 A$ & COND & 1991Q1 & & 15 USCM \\
\hline ABP $1 A$ & COND & 1991Q1 & & 15 USCM \\
\hline ABP IA & COND & 1991Q2 & & 16 USCM \\
\hline$A B P \quad 1 A$ & COND & $1991 Q 3$ & & 20 USCM \\
\hline ABP $1 A$ & COND & $1991 Q 3$ & & 20 USCM \\
\hline$A B P$ IA & COND & 1991Q4 & & 18 USCM \\
\hline$A B P$ IA & COND & 1992Q1 & & 11 USCM \\
\hline$A B P 1 A$ & COND & 1992Q1 & & 11 USCM \\
\hline$A B P 1 A$ & COND & $1992 Q 2$ & & 15 USCM \\
\hline ABP $1 A$ & COND & 1992Q2 & & 15 USCM \\
\hline ABP $1 A$ & COND & 1992Q3 & & 18 USCM \\
\hline ABP $1 A$ & COND & 199302 & & 13 USCM \\
\hline ABP IA & COND & 1993Q3 & & 14 USCM \\
\hline$A B P \quad 1 A$ & COND & 1993Q4 & & 13 USCM \\
\hline ABP $1 A$ & COND & 1994Q1 & & 12 USCM \\
\hline ABP IA & COND & 1994Q2 & & 13 USCM \\
\hline ABP $1 A$ & COND & 1994Q3 & & 13 USCM \\
\hline$A B P$ IA & COND & 1995Q1 & & 12 USCM \\
\hline$A B P$ 1A & COND & 1996Q1 & & 16 USCM \\
\hline$A B P$ IA & COND & 1996Q2 & & 12 USCM \\
\hline ABP $1 A$ & COND & 199704 & & 12 USCM \\
\hline$A B P 1 A$ & COND & 1998Q3 & & 18 USCM \\
\hline$A B P \quad 1 A$ & COND & 1999Q3 & & 13 USCM \\
\hline
\end{tabular}

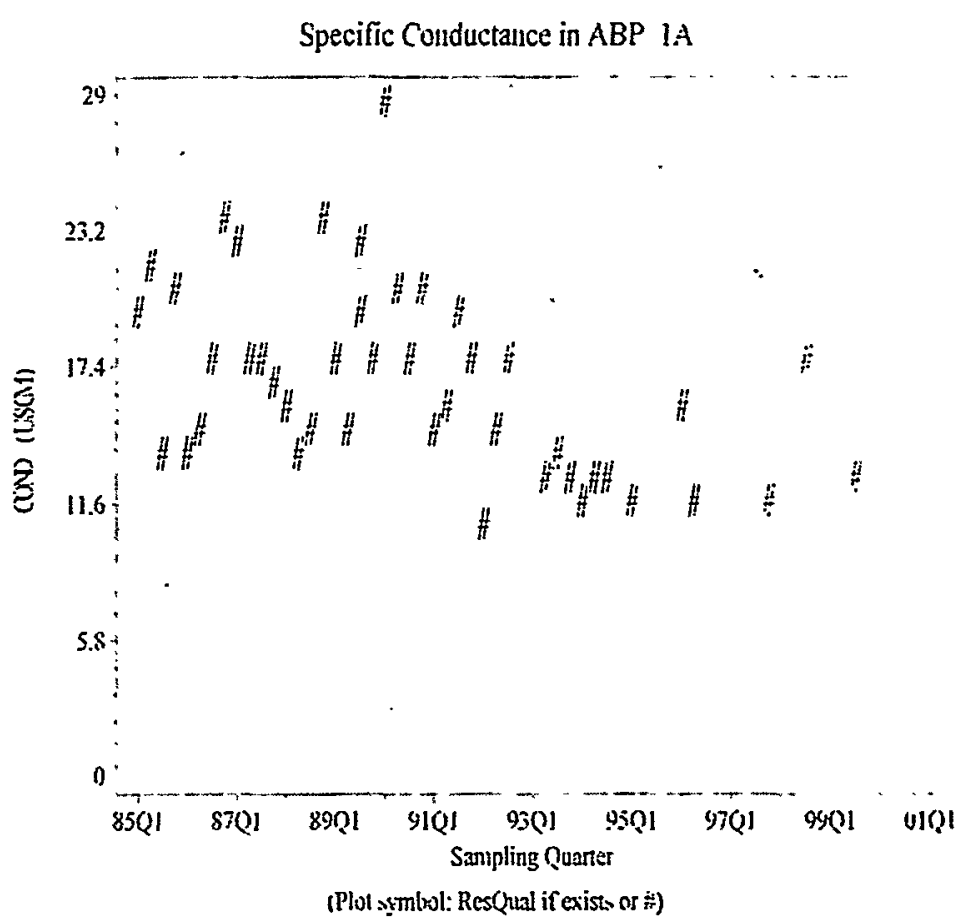

(Plol symlool: Res(qual if existo os 
Calcium, total recoverable in $\mathrm{ABP} 1 \mathrm{~A}$

Data Retrieved by AWSA v1 from GIMS on 09NOV99 at 15:50

Well Analyte Sample_Q Res_Qual Result Units

ABP 1A CATOT 199601

ABP IA CATOT 1996Q1

ABP 1A CATOT 199622

ABP 1A CATOT 1997Q4

ABP 1A CATOT 1998Q3

ABP 1A CATOT 199803

ABP $1 A$ CATOT 1999Q3
432 UGL
437 UGL
748 UGL
532 UGL
572 UGL
597 UGL
516 UGL

149.6

0

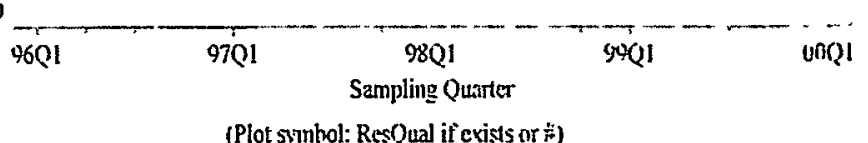


Potassium, total recoverable in ABP $1 A$

Data Retrieved by AWSA v1 from GIMS on 09NOV99 at 15:50

\begin{tabular}{llrlr} 
Well & Analyte & \multicolumn{2}{c}{ Sample_Q Res_Qual Result } & Units \\
& & & & \\
ABP 1A & KTOT & $1996 Q 1$ & $\mathrm{~J}$ & 217 UGL \\
ABP 1A & KTOT & $1996 Q 1$ & $\mathrm{~J}$ & 217 UGL \\
ABP 1A & KTOT & $1996 Q 2$ & U & 2000 UGL \\
ABP 1A & KTOT & $1997 Q 4$ & 271 UGL \\
ABP 1A & KTOT & $1998 Q 3$ & 238 UGL \\
ABP 1A & KTOT & $1998 Q 3$ & 252 UGL \\
ABP 1A & KTOT & $1999 Q 3$ & 223 UGL
\end{tabular}

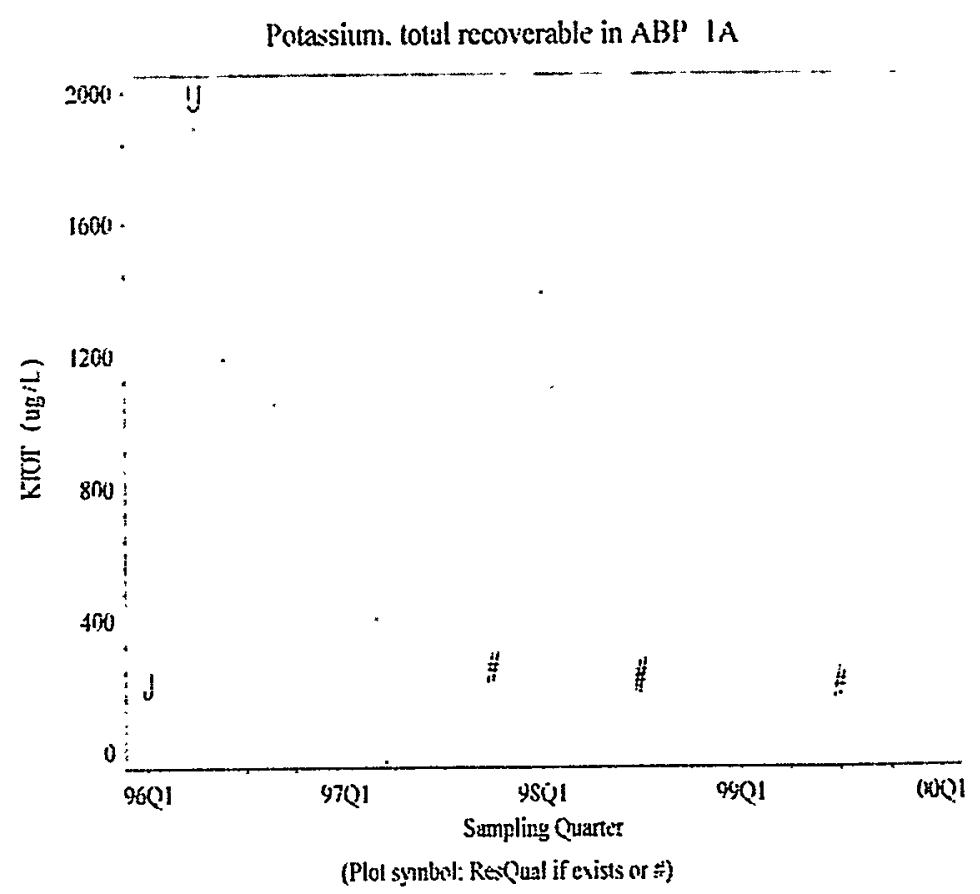


Sodium, total recoverable in ABP $1 \mathrm{~A}$

Data Retrieved by AWSA v1 from GIMS on 09NOV99 at 15:50

Well Analyte Sample_Q Res_Qual Result Units

ABP 1A NATOT 199601

ABP IA NATOT 199601

ABP 1A NATOT 199622

ABP IA NATOT 1997Q4

ABP 1A NATOT $1998 \mathrm{~B}$

ABP IA NATOT 1998Q3

ABP $1 A$ NATOT $1999 Q 3$
958 UGL 969 UGL 1350 UGL 1020 UGL 856 UGL 900 UGL 821 UGL

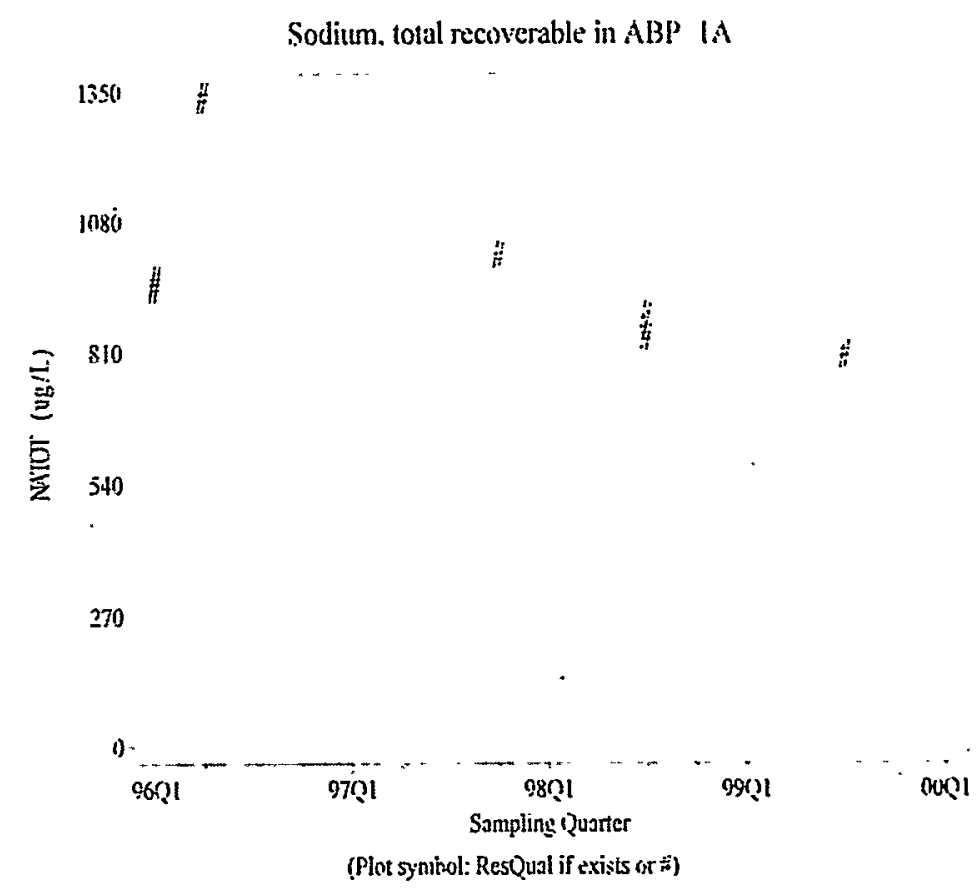


Chromium, total recoverable in ABP $1 \mathrm{~A}$

Data Retrieved by AWSA v1 from GIMS on 09NOV99 at 15:50

\begin{tabular}{llllr} 
Well & Analyte & \multicolumn{2}{c}{ Sample_Q Res_Qual Result } & Units \\
& & & & \\
ABP 1A & CRTOT & $1995 Q 1$ & $U$ & 6.67 UGL \\
ABP 1A & CRTOT & $1996 Q 1$ & & 4.4 UGL \\
ABP 1A & CRTOT & $1996 Q 1$ & & 6.42 UGL \\
ABP 1A & CRTOT & $1996 Q 2$ & $U$ & 25 UGL \\
ABP 1A & CRTOT & $1997 Q 4$ & $U$ & 5 UGL \\
ABP 1A & CRTOT & $1998 Q 3$ & $U$ & 3.9 UGL \\
ABP 1A & CRTOT & $1998 Q 3$ & U & 6.35 UGL \\
ABP 1A & CRTOT & $1999 Q 3$ & J & 2.25 UGL
\end{tabular}
67 UGL 4.4 UGL 5 UGL 5 UGL 6.35 UGL 25 UGL

20

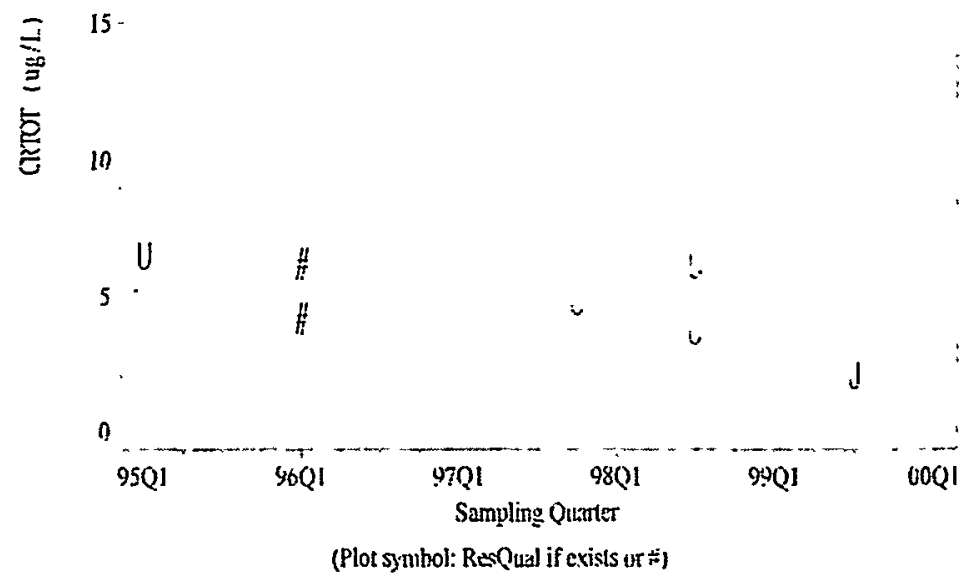


Iron, total recoverable in ABP $1 \mathrm{~A}$

Data Retrieved by AWSA v1 from GIMS on 09NOV99 at 15:50

Well Analyte Sample_Q Res_Qual Result Units

ABP 1A FETOT 1996Q1 J 64.2 UGL

ABP 1A FETOT 1996Q1 J 78.9 UGL

ABP 1A FETOT $1996 Q 2$ J 14.9 UGL

ABP 1A FETOT $199803 \quad 44$ UGL

ABP IA FETOT $1998 \mathrm{Q} 3 \quad 52.2 \mathrm{UGL}$

ABP 1A FETOT $1999 Q 3 U$ U 50 UGL

Iron, total recoverable in $A B P$ IA

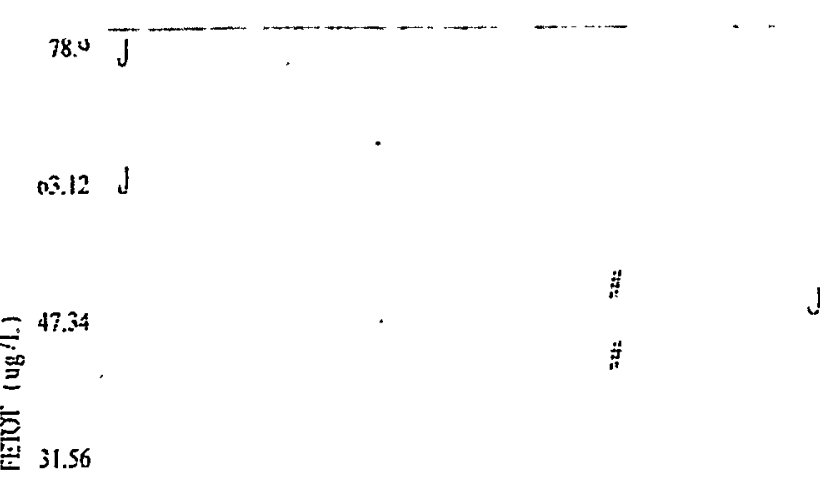

$15.78 \quad J$

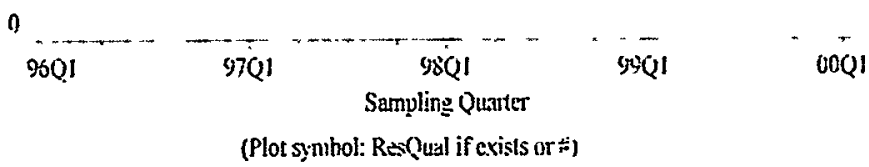


Manganese, total recoverable in ABP $1 \mathrm{~A}$

Data Retrieved by AWSA v1 from GIMS on 09NOV99 at 15:50

Well Analyte Sample_Q Res_Qual Result Units

ABP 1A MNTOT $199303 \quad U$

ABP 1A MNTOT 1994Q1 J

ABP 1A MNTOT $1994 Q 3 \quad J$

ABP 1A MNTOT 1995Q1 J

ABP 1A MNTOT 1996Q1

ABP 1A MNTOT 199601

ABP 1A MNTOT 1996Q1 J

ABP 1A MNTOT 1996Q2 U

ABP 1A MNTOT 1997Q4 J

ABP 1A MNTOT 1998Q3 U

ABP 1A MNTOT 1998Q3 U

ABP 1A MNTOT 1999 Q3
2 UGL

2.71 UGL 3.32 UGL 2.28 UGL 2.56 UGL 3,07 UGL 4.48 UGL 10 UGL 3.14 UGL 10 UGL 10 UGL 10 UGL

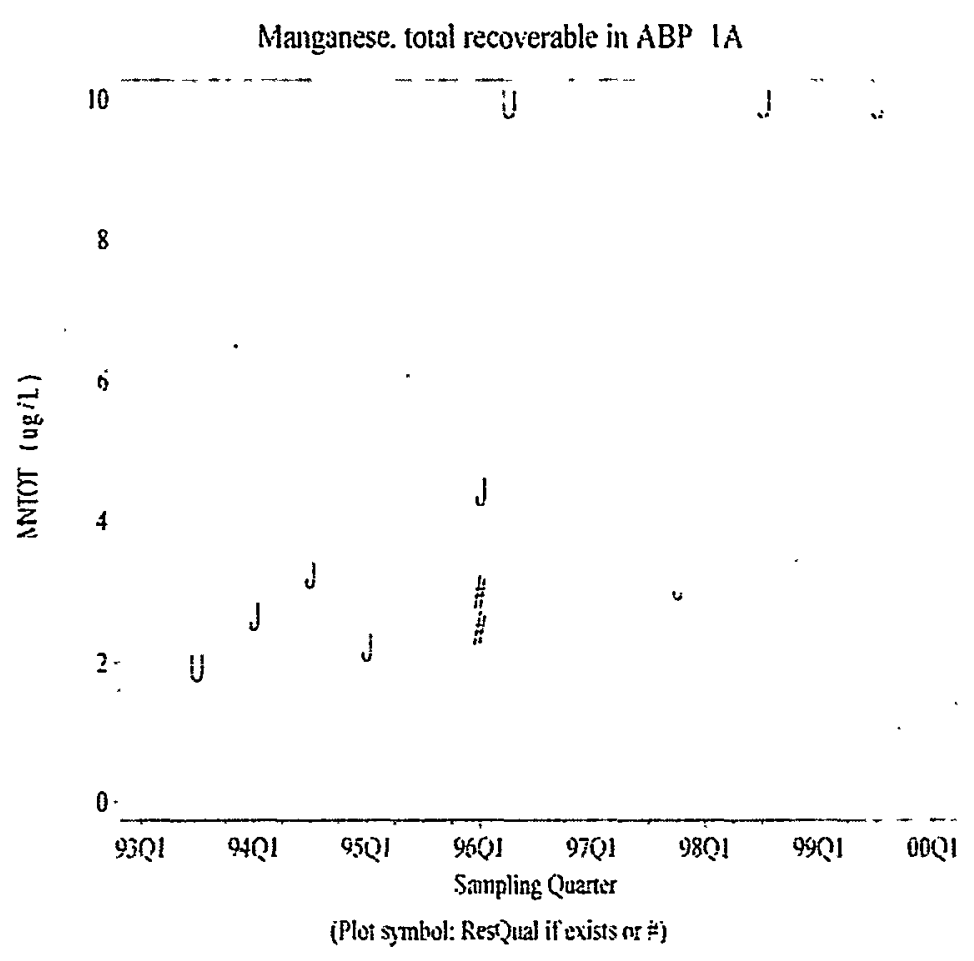


Aluminum, total recoverable in ABP $1 \mathrm{~A}$

Data Retrieved by AWSA v1 from GIMS on 09NOV99 at 15:50

Well Analyte Sample_Q Res_Qual Result Units

ABP 1A ALTOT $199303 \quad U \quad 20$ UGL

ABP 1A ALTOT 1994Q1 $U$ 20 UGL

ABP 1A ALTOT $199403 \cup \quad 20$ UGL

ABP 1A ALTOT 199501 U $33.3 \mathrm{UGL}$

ABP 1A ALTOT $1996 \mathrm{Q} 1 \mathrm{U} \quad 22.9$ UGL

ABP 1A ALTOT 199601 $\cdot U$ 46.4 UGL

ABP 1A ALTOT 1996Q2 U 250 UGL

ABP IA ALTOT 1997Q4 U 50 UGL

ABP 1A ALTOT 1998Q3 U

ABP IA ALTOT 1998Q3 U 23.8 UGL

ABP IA ALTOT 1999Q3 U 50 UGL
Aluminum, total recoverable in ABP IA

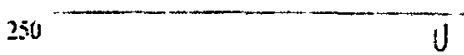

200

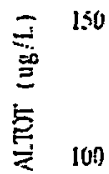

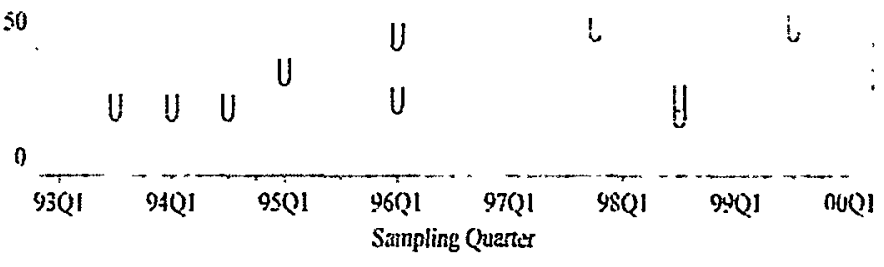

(l'lot symbol. ResQual if exisss or 
Lead, total recoverable in ABP IA

Data Retrieved by AWSA v1 from GIMS on 09NOV99 at $15: 50$

Well Analyte Sample_Q Res_Qual Result Units

ABP 1A PBTOT 1993Q3 U 3 UGL

ABP 1A PBTOT 1994Q1 U 3 UGL

ABP 1A PBTOT 1994Q3 U 3 UGL

ABP $1 A$ PBTOT 1995Q1 U 5 UGL

ABP IA PBTOT 1996Q1 U .1.19 UGL

ABP 1A PBTOT 1996Q1 U 2.23 UGL

ABP 1A PBTOT 1996Q1 U $100 \mathrm{UGL}$

ABP 1A PBTOT $199602 \quad 0.371$ UGL

ABP IA PBTOT 1997Q4 J 2.9 UGL

ABP IA PBTOT 1998Q3 U 2 UGL

ABP 1A PBTOT 1998Q3 U 2 UGL

ABP 1A PBTOT 1999Q3 U 5 UGL

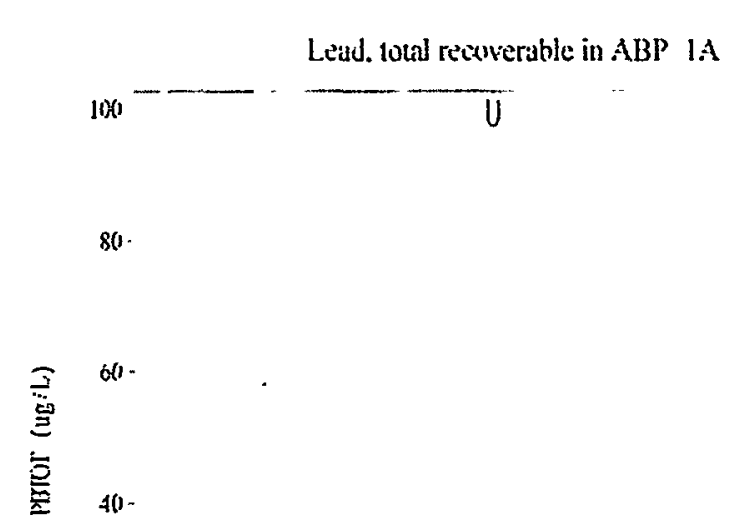

20 -

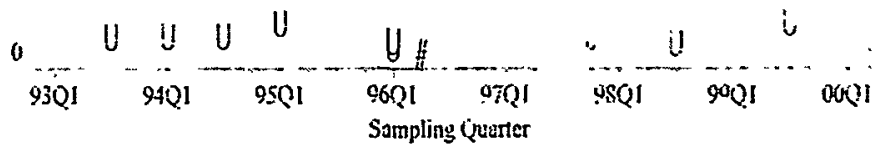

(Plot symbul. Res(ual if exists or :) 
Chloride in ABP $1 \mathrm{~A}$

Data Retrieved by AWSA v1 from GIMS on 09NOV99 at 15:50

\begin{tabular}{|c|c|c|c|c|}
\hline Well & Analyte & Sample_Q Res_Qual & Result & Units \\
\hline$A B P \quad 1 A$ & $\mathrm{CL}$ & 1985Q1 & 1140 & 0 UGL \\
\hline$A B P$ IA & $\mathrm{CL}$ & 1985Q2 & 1980 & 0 UGL \\
\hline ABP 1A & $\mathrm{CL}$ & $1985 Q 3$ & 1600 & 0 UGL \\
\hline$A B P 1 A$ & CL & 198504 & 2350 & 0 UGL \\
\hline$A B P 1 A$ & $\mathrm{CL}$ & 1985Q4 & 2350 & 0 UGL \\
\hline ABP $1 A$ & $\mathrm{CL}$ & 1986Q1 & 6650 & 0 UGL \\
\hline$A B P 1 A$ & $\mathrm{CL}$ & 1987Q1 & 2000 & 0 UGL \\
\hline$A B P 1 A$ & $\mathrm{CL}$ & 198902 & 4000 & 0 UGL \\
\hline$A B P 1 A$ & $\mathrm{CL}$ & $1989 Q 2$ & 4000 & 0 UGL \\
\hline$A B P 1 A$ & $\mathrm{CL}$ & $1989 Q 3$ & 1300 & 0 UGL \\
\hline$A B P 1 A$ & $\mathrm{CL}$ & $1990 Q 3$ & 1340 & 0 UGL \\
\hline$A B P \quad 1 A$ & $\mathrm{CL}$ & 199003 & 1370 & 0 UGL \\
\hline$A B P 1 A$ & $\mathrm{CL}$ & 199003 & 1400 & 0 UGL \\
\hline$A B P 1 A$ & $\mathrm{CL}$ & 199003 & 1400 & 0 UGL \\
\hline ABP $1 A$ & $\mathrm{CL}$ & 199003 & 1400 & 0 UGL \\
\hline ABP $1 A$ & $\mathrm{CL}$ & 199004 & 1400 & 0 UGL \\
\hline$A B P \quad 1 A$ & $\mathrm{CL}$ & $1990 Q 4$ & 1400 & 0 UGL \\
\hline$A B P 1 A$ & $\mathrm{CL}$ & 1981Q1 & 1310 & 0 UGL \\
\hline ABP $1 A$ & $\mathrm{CL}$ & 1991Q1 & 1320 & 0 UGL \\
\hline$A B P 1 A$ & $\mathrm{CL}$ & 1991Q1 & 1330 & 0 UGL \\
\hline$A B P$ IA & $\mathrm{CL}$ & 1991Q1 & 1360 & 0 UGL \\
\hline$A B P 1 A$ & $\mathrm{CL}$ & 1991Q1 & 1420 & 0 UGL \\
\hline ABP $1 A$ & $\mathrm{CL}$ & 199102 & 3730 & 0 UGL \\
\hline$A B P$ IA & $\mathrm{CL}$ & $1991 Q 3$ & 1590 & 0 UGL \\
\hline$A B P \quad 1 A$ & $C L$ & 1991Q3 & 1640 & 0 UGL \\
\hline$A B P$ 1A & $C L$ & 1991Q4 & 1520 & 0 UGL \\
\hline$A B P 1 A$ & $C L$ & 1992Q1 & 1550 & 0 UGL \\
\hline$A B P 1 A$ & $\mathrm{CL}$ & $1997 Q 4$ & 1600 & 0 UGL \\
\hline$A B P \quad 1 A$ & $\mathrm{CL}$ & 1997Q4 & 1660 & 0 UGL \\
\hline$A B P 1 A$ & CL & $1998 Q 3$ & 1520 & 0 UGL \\
\hline$A B P \quad 1 A$ & $\mathrm{Cl}$ & 199803 & 1560 & 0 UGL \\
\hline ABP $1 A$ & $\mathrm{CL}$ & 1999Q3 & 1400 & 0 UGL \\
\hline
\end{tabular}

Chloride in ABP 1A

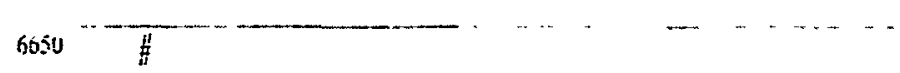

$5 \hat{2} 20$

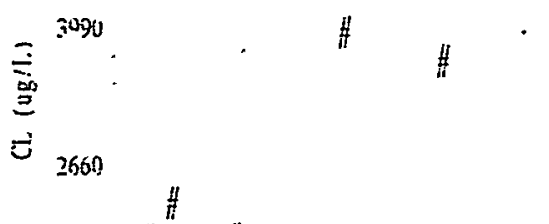

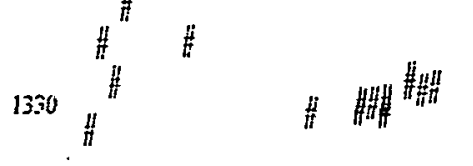

H

)

$8501 \quad 8701$ 89Q1 9101 9301 9501 9701 99Q1
Simpling (uarter

- (lio symbol: ResQual if exists or 


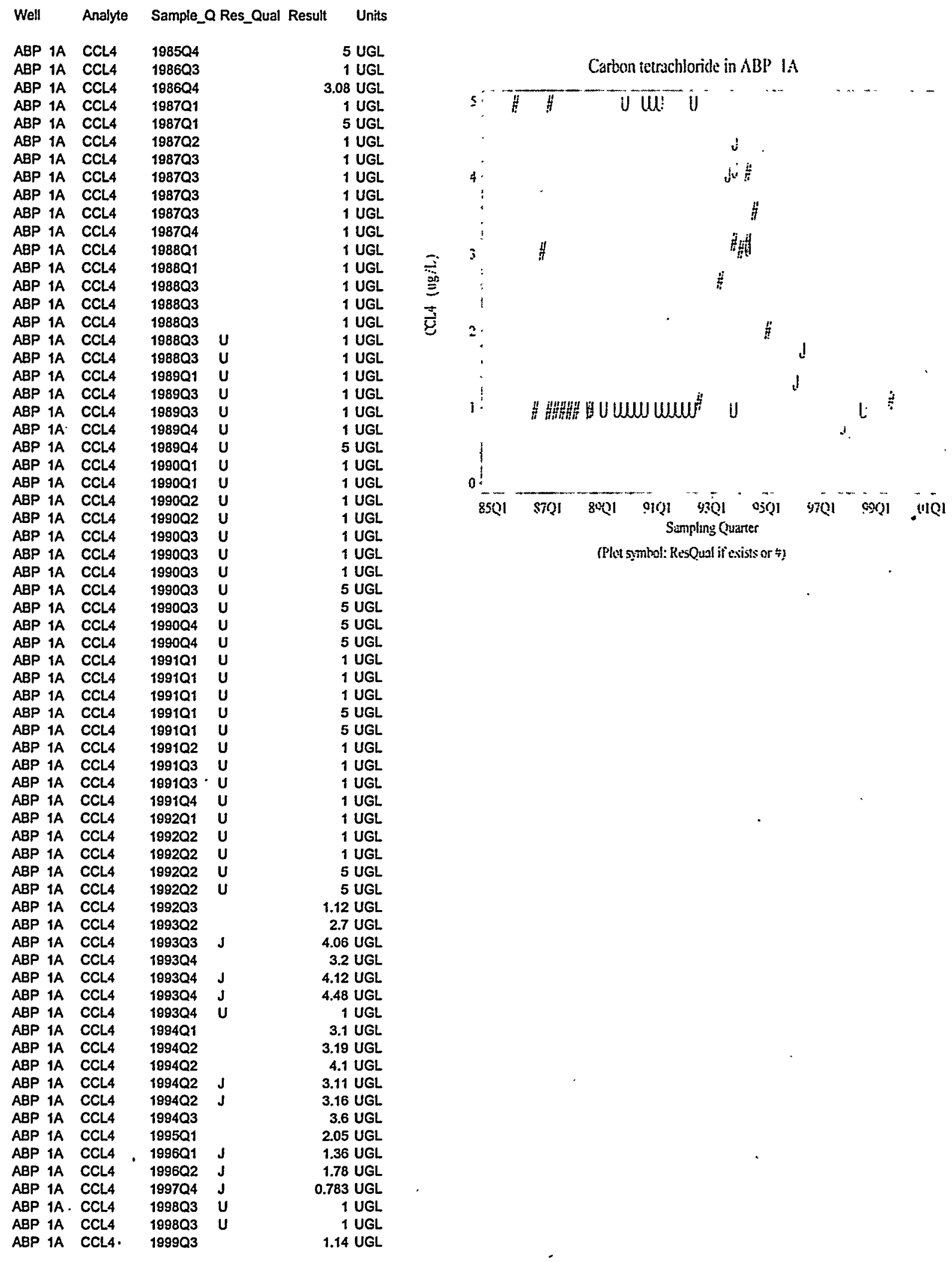


Tetrachloroethylene in ABP $1 \mathrm{~A}$

Data Retrieved by AWSA v1 from GIMS on 09NOV99 at 15:50

\begin{tabular}{|c|c|c|c|c|}
\hline Well & Analyte & Sample_Q & Res_Qual Result & Units \\
\hline$A B P \quad 1 A$ & TCLEE & $1992 Q 2$ & $J$ & 2.16 UGL \\
\hline ABP $1 A$ & TCLEE & $1992 Q 3$ & & 6.52 UGL \\
\hline$A B P 1 A$ & TCLEE & 199302 & & 15.4 UGL \\
\hline$A B P 1 A$ & TCLEE & 199303 & $J$ & 19.4 UGL \\
\hline$A B P \quad 1 A$ & TCLEE & 199324 & & 17.4 UGL \\
\hline ABP $1 A$ & TCLEE & 199304 & & 19.3 UGL \\
\hline ABP $1 A$ & TCLEE & 199304 & & 20.7 UGL \\
\hline$A B P \quad 1 A$ & TCLEE & $1993 Q 4$ & $U$ & 1 UGL \\
\hline$A B P \quad 1 A$ & TCLEE & 1994Q1 & & $23.6 \mathrm{UGL}$ \\
\hline$A B P \quad 1 A$ & TCLEE & 199402 & & $15 \mathrm{UGL}$ \\
\hline ABP $1 A$ & TCLEE & 199402 & & 15.4 UGL \\
\hline ABP $1 A$ & TCLEE & $1994 Q 2$ & & 16.2 UGL \\
\hline ABP $1 A$ & TCLEE & 199402 & & 17.2 UGL \\
\hline ABP $1 A$ & TCLEE & 199403 & & 15.9 UGL \\
\hline ABP $1 A$ & TCLEE & 1995Q1 & & 10.2 UGL \\
\hline$A B P 1 A$ & TCLEE & 199601 & & 9.13 UGL \\
\hline$A B P 1 A$ & TCLEE & 199602 & & 8.97 UGL \\
\hline ABP $1 A$ & TCLEE & $1997 Q 4$ & & 5.16 UGL \\
\hline$A B P 1 A$ & TCLEE & $1998 Q 3$ & & 1.59 UGL \\
\hline ABP $1 A$ & TCLEE & $1998 Q 3$ & $u$ & 1 UGL \\
\hline$A B P \quad 1 A$ & TCLEE & 1999Q3 & & 8.8 UGL \\
\hline
\end{tabular}


Trichloroethylene in ABP $1 \mathrm{~A}$

Data Retrieved by AWSA vi from GIMS on 09NOV99 at 15:50

\begin{tabular}{|c|c|c|c|c|}
\hline Well & Analyte & Sample_c & Res_Qual & Result \\
\hline$A B P \quad 1 A$ & TRCLE & $1985 Q 4$ & & 5 UGL \\
\hline$A B P 1 A$ & TRCLE & 1986Q2 & & 2.51 UGL \\
\hline ABP $1 A$ & TRCLE & $1986 Q 3$ & & $2.08 \mathrm{UGL}$ \\
\hline$A B P 1 A$ & TRCLE & $1986 Q 4$ & & 3.73 UGL \\
\hline$A B P 1 A$ & TRCLE & 1987Q1 & & 1 UGL \\
\hline ABP $1 A$ & TRCLE & 1987Q1 & & 5 UGL \\
\hline$A B P 1 A$ & TRCLE & $1987 Q 2$ & & 1 UGL \\
\hline ABP $1 A$ & TRCLE & 1987Q3 & & 1 UGL \\
\hline ABP $1 A$ & TRCLE & 1987Q3 & & 1 UGL \\
\hline$A B P 1 A$ & TRCLE & 1987Q3 & & 1 UGL \\
\hline$A B P \quad 1 A$ & TRCLE & 1987Q3 & & 1 UGL \\
\hline$A B P 1 A$ & TRCLE & $1987 Q 4$ & & 1 UGL \\
\hline ABP $1 A$ & TRCLE & 1988Q1 & & 1 UGL \\
\hline$A B P 1 A$ & TRCLE & 1988Q1 & & 1 UGL \\
\hline$A B P 1 A$ & TRCLE & 198802 & & 1 UGL \\
\hline$A B P 1 A$ & TRCLE & 1988Q3 & & 1 UGL \\
\hline$A B P 1 A$ & TRCLE & $1988 Q 3$ & & 1 UGL \\
\hline$A B P 1 A$ & TRCLE & $1988 Q 3$ & & 1 UGL \\
\hline$A B P 1 A$ & TRCLE & $1988 Q 3$ & & 1.5 UGL \\
\hline ABP $1 A$ & TRCLE & $1888 Q 3$ & & 2 UGL \\
\hline ABP $1 A$ & TRCLE & $1988 Q 3$ & & 2 UGL \\
\hline$A B P 1 A$ & TRCLE & $1988 Q 3$ & $U$ & 1 UGL \\
\hline ABP $1 \mathrm{~A}$ & TRCLE & 1988Q4 & & 1.3 UGL \\
\hline ABP $1 A$ & TRCLE & 1989Q1 & & 1.05 UGL \\
\hline ABP $1 A$ & TRCLE & 1989Q1 & & 2.5 UGL \\
\hline$A B P 1 A$ & TRCLE & 1989Q1 & & 2.97 UGL \\
\hline ABP $1 A$ & TRCLE & $1989 Q 2$ & & 1.55 UGL \\
\hline$A B P 1 A$ & TRCLE & $1989 Q 3$ & & 1 UGL \\
\hline $\mathrm{ABP} 1 \mathrm{~A}$ & TRCLE & $1989 Q 3$ & & 1 UGL \\
\hline$A B P 1 A$ & TRCLE & $1989 Q 3$ & & 1.32 UGL \\
\hline$A B P 1 A$ & TRCLE & 198904 & & 1.07 UGL \\
\hline$A B P 1 A$ & TRCLE & $1989 Q 4$ & U & 1 UGL \\
\hline$A B P 1 A$ & TRCLE & $1989 Q 4$ & $U$ & 1 UGL \\
\hline ABP $1 A$ & TRCLE & $1989 Q 4$ & U & 5 UGL \\
\hline$A B P 1 A$ & TRCLE & 1990Q1 & U. & 1 UGL \\
\hline$A B P 1 A$ & TRCLE & $1990 Q 1$ & $u$ & 1 UGL \\
\hline$A B P 1 A$ & TRCLE & 199001 & $U$ & 1 UGL \\
\hline$A B P \quad 1 A$ & TRCLE & 199002 & & 0.58 UGL \\
\hline$A B P 1 A$ & TRCLE & $1990 Q 2$ & $u$ & 1 UGL \\
\hline $\mathrm{ABP} 1 \mathrm{~A}$ & TRCLE & 199002 & $u$ & 1 UGL \\
\hline ABP $1 \mathrm{~A}$ & TRCLE & $1990 Q 2$ & $\mathbf{u}$ & 1 UGL \\
\hline$A B P \quad 1 A$ & TRCLE & $1990 Q 3$ & & 1 UGL \\
\hline$A B P$ 1A & TRCLE & $1990 Q 3$ & & 1 UGL \\
\hline$A B P 1 A$ & TRCLE & $1990 Q 3$ & & 1.15 UGL \\
\hline$A B P 1 A$ & TRCLE & $1990 Q 3$ & $u$ & 1 UGL \\
\hline$A B P \quad 1 A$ & TRCLE & $90 Q 3$ & $u$ & 1 UGL \\
\hline$A B P 1 A$ & TRCLE & $1990 Q 3$ & $u$ & 10 UGL \\
\hline$A B P 1 A$ & TRCLE & $1990 Q 3$ & $u$ & 10 UGL \\
\hline$A B P \quad 1 A$ & TRCLE & $1990 Q 4$ & $U$ & 1 UGL \\
\hline$A B P 1 A$ & TRCLE & $1990 Q 4$ & $u$ & 5 UGL \\
\hline ABP $1 A$ & TRCLE & $1990 Q 4$ & $u$ & 5 UGL \\
\hline ABP $1 \mathrm{~A}$ & TRCLE & 1991Q1 & $\mathbf{U}$ & 1 UGL \\
\hline ABP $1 A$ & TRCLE & 1991Q1 & $u$ & 1 UGL \\
\hline$A B P 1 A$ & TRCLE & 1991Q1 & u & 1 UGL \\
\hline ABP $1 A$ & TRCLE & 1991Q1 & $u$ & 10 UGL \\
\hline ABP $1 \mathrm{~A}$ & TRCLE & 1991Q1 & $\mathbf{u}$ & 10 UGL \\
\hline ABP $1 \mathrm{~A}$ & TRCLE & 1991Q2 & U & 1 UGL \\
\hline $\mathrm{ABP} 1 \mathrm{~A}$ & TRCLE & 1991Q3 & & 1 UGL \\
\hline$A B P 1 A$ & TRCLE & 1991Q3 & $U$ & 1 UGL \\
\hline$A B P 1 A$ & TRCLE & 1991Q4 & & 2 UGL \\
\hline ABP $1 A$ & TRCLE & 1992Q1 & & 2.29 UGL \\
\hline$A B P 1 A$ & TRCLE & 199202 & & 5.05 UGL \\
\hline$A B P 1 A$ & TRCLE & 199202 & & 5.37 UGL \\
\hline
\end{tabular}

Trichloroethylene in ABP $\mathrm{AA}$

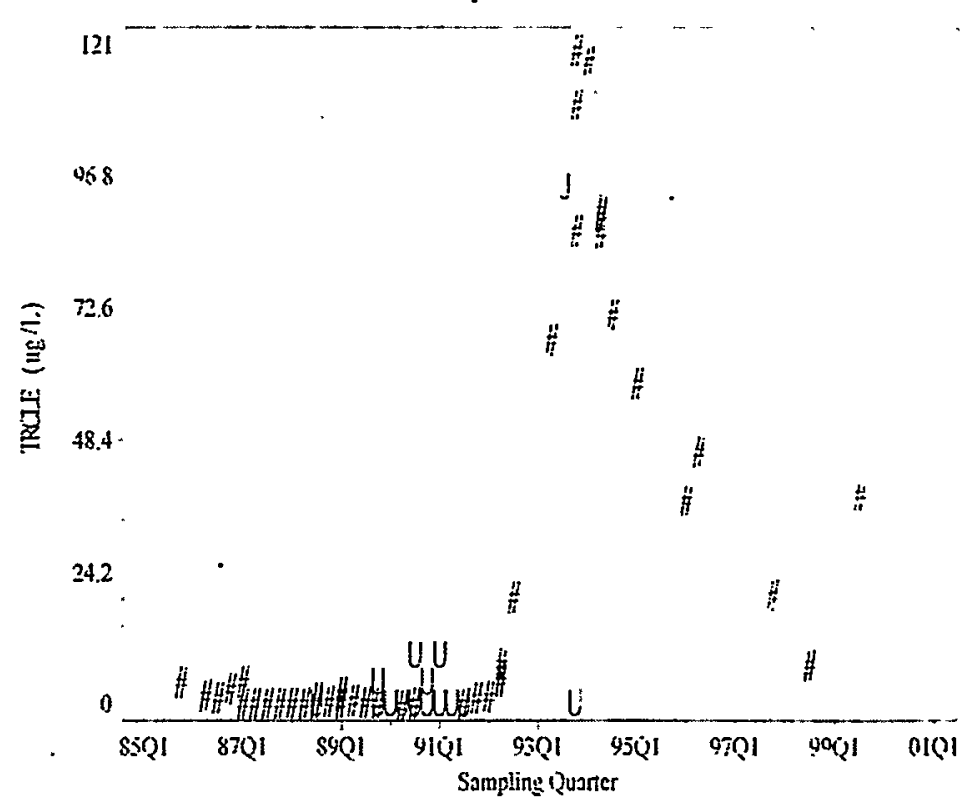

(Plot synibol: ResQual if exists or 
Trichloroethylene in ABP $1 \mathrm{~A}$

Data Retrieved by AWSA v1 from GIMS on 09NOV99 at 15:50

\begin{tabular}{|c|c|c|c|c|c|}
\hline Wel! & Analyte & Sample_C & Res_Qual & Result & Units \\
\hline$A B P 1 A$ & TRCLE & 1992Q2 & & & .09 UGL \\
\hline$A B P$ IA & TRCLE & 199202 & & & 28 UGL \\
\hline$A B P 1 A$ & TRCLEE & $1992 Q 3$ & & & 0.7 UGL \\
\hline$A B P\{A$ & TRCLE & $1993 Q 2$ & & 67. & 7.9 UGL \\
\hline$A B P \quad 1 A$ & TRCLE & 1993Q3 & $J$ & 95. & 5.9 UGL \\
\hline$A B P$ IA & TRCLE & $1993 Q 4$ & & 87. & 7.8 UGL \\
\hline$A B P$ 1A & TRCLE & $1993 Q 4$ & & & 11 UGL \\
\hline ABP $1 A$ & TRCLE & $1993 Q 4$ & & & 21 UGL \\
\hline$A B P 1 A$ & TRCLE & $1993 Q 4$ & $u$ & & 1 UGL \\
\hline$A B P 1 A$ & TRCLE & 1994Q1 & & & 119 UGL \\
\hline ABP $1 A$ & TRCLE & $1994 Q 2$ & & & 7.5 UGL \\
\hline ABP $1 A$ & TRCLE & $1994 Q 2$ & & & 0.5 UGL \\
\hline$A B P 1 A$ & TRCLE & 1994Q2 & & & 0.9 UGL \\
\hline$A B P 1 A$ & TRCLE & 1994Q2 & & & $1.6 \mathrm{UGL}$ \\
\hline ABP 1A & TRCLE & 1994Q3 & & & 2.4 UGL \\
\hline$A B P 1 A$ & TRCLE & 1995Q1 & & & 9.9 UGL \\
\hline ABP $1 A$ & TRCLE & 1996Q1 & & & 8.4 UGL \\
\hline ABP $1 A$ & TRCLE & $1996 Q 2$ & & & 7.2 UGL \\
\hline ABP $1 A$ & TRCLE & $1997 Q 4$ & & & 1.1 UGL \\
\hline$A B P$ 1A & TRCLE & $1998 Q 3$ & & & .24 UGL \\
\hline ABP 1A & TRCLE & 1998Q3 & & & .28 UGL \\
\hline ABP $1 A$ & TRCLE & $1999 Q 3$ & & & 9.6 UGL \\
\hline
\end{tabular}


Gross alpha in ABP IA

Data Retrieved by AWSA v1 from GIMS on 09NOV99 at 15:50

\begin{tabular}{|c|c|c|c|c|}
\hline Well & Analyte & Sample_C & I Res_Qual & Result \\
\hline ABP $1 \mathrm{~A}$ & ALPHAG & 1985Q1 & & $2.1 \mathrm{PCL}$ \\
\hline$A B P \quad 1 A$ & ALPHAG & 1985Q2 & & $2 \mathrm{PCL}$ \\
\hline$A B P$ 1A & ALPHAG & 198503 & & $2 \mathrm{PCL}$ \\
\hline ABP $1 A$ & ALPHAG & $1985 Q 4$ & & 2 PC \\
\hline$A B P 1 A$ & ALPHAG & 1986Q1 & & $2 P C$ \\
\hline$A B P \quad 1 A$ & ALPHAG & 1987Q1 & & $3 P C$ \\
\hline$A B P 1 A$ & ALPHAG & 1988Q1 & & $1.1 \mathrm{P}$ \\
\hline$A B P 1 A$ & ALPHAG & .1988Q1 & & $1.1 \mathrm{PC}$ \\
\hline$A B P 1 A$ & ALPHAG & 1989Q1 & & $0.96 \mathrm{PCl}$ \\
\hline$A B P 1 A$ & ALPHAG & 1989Q2 & $\mathbf{U}$ & $3 \mathrm{PCL}$ \\
\hline ABP $1 A$ & ALPHAG & 1989Q2 & $u$ & $3 \mathrm{PCL}$ \\
\hline ABP $1 \mathrm{~A}$ & ALPHAG & $1989 Q 3$ & $U$ & $2 \mathrm{PC}$ \\
\hline $\mathrm{ABP} \quad 1 \mathrm{~A}$ & ALPHAG & $1990 Q 1^{\circ}$ & $u$ & \\
\hline ABP $1 A$ & ALPHAG & 1990Q1 & $U$ & $3 P C$ \\
\hline ABP $1 \mathrm{~A}$ & ALPHAG & $1990 Q 3$ & $U$ & $2 \mathrm{PC}$ \\
\hline ABP $1 \mathrm{~A}$ & ALPHAG & 1990Q3 & $U$ & $2 \mathrm{PC}$ \\
\hline ABP $1 A$ & ALPHAG & $1990 Q 3$ & U & \\
\hline $\mathrm{ABP} 1 \mathrm{~A}$ & ALPHAG & 1990Q3 & $U$ & \\
\hline ABP $1 A$ & ALPHAG & 199003 & $u$ & 28 \\
\hline ABP $1 A$ & ALPHAG & $1990 Q 4$ & $\mathbf{U}$ & \\
\hline ABP $1 A$ & ALPHAG & $1990 Q 4$ & $U$ & $2 \mathrm{P}$ \\
\hline ABP $1 A$ & ALPHAG & 1991Q1 & & $5.8 \mathrm{PC}$ \\
\hline$A B P \quad 1 A$ & ALPHAG & 1991Q1 & $U$ & $2 P C$ \\
\hline ABP $1 \mathrm{~A}$ & ALPHAG & 1991Q1 & $U$ & $2 f$ \\
\hline ABP $1 A$ & ALPHAG & $1991 Q 1$ & $U$ & \\
\hline ABP $1 A$ & ALPHAG & 1991Q1 & $U$ & \\
\hline ABP $1 A$ & ALPHAG & 1991Q1 & $u$ & $3 P C$ \\
\hline$A B P 1 A$ & ALPHAG & 1991Q2 & $U$ & $2 P$ \\
\hline ABP $1 A$ & ALPHAG & 1991Q3 & $\mathbf{u}$ & $2 P$ \\
\hline$A B P 1 A$ & ALPHAG & 1991Q3 & $\mathbf{u}$ & $2 P$ \\
\hline$A B P 1 A$ & ALPHAG & 199104 & $u$ & $2 \mathrm{PCL}$ \\
\hline$A B P 1 A$ & ALPHAG & 1992Q1 & $\mathbf{U}$ & \\
\hline ABP $1 A$ & ALPHAG & 1994Q3 & UI & $0.779 \mathrm{PC}$ \\
\hline ABP $1 A$ & ALPHAG & 1994Q3 & UI & $1.11 P C$ \\
\hline ABP $1 A$ & ALPHAG & 1995Q1 & & $0.808 \mathrm{PC}$ \\
\hline $\mathrm{ABP} 1 \mathrm{~A}$ & ALPHAG & 1996Q1 & & $0.711 P C$ \\
\hline ABP $1 A$ & ALPHAG & $1996 Q 2$ & & $0.783 \mathrm{PC}$ \\
\hline ABP $1 A$ & ALPHAG & $1997 Q 4$ & & \\
\hline ABP $1 A$ & ALPHAG & 1998Q3 & $\mathbf{U}$ & 0.657 \\
\hline ABP $1 A$ & ALPHAG & 1998Q3 & $\mathbf{U}$ & \\
\hline$A B P 1 A$ & ALPHAG & 1999Q3 & J & \\
\hline
\end{tabular}

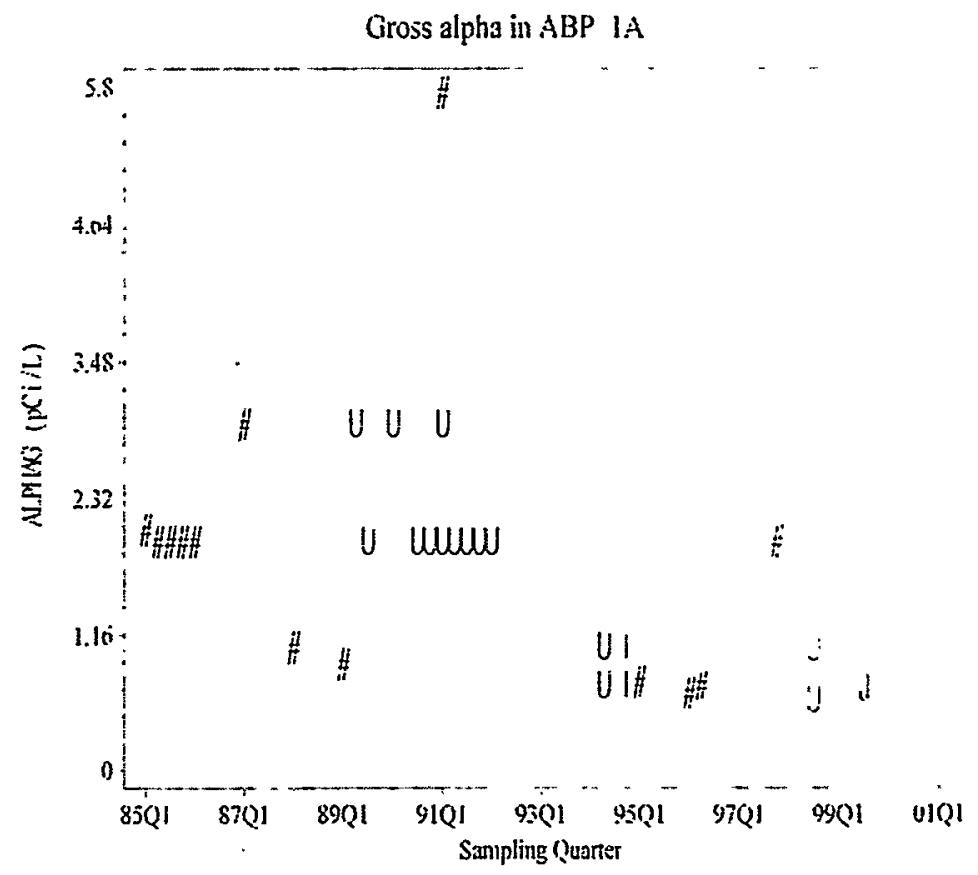

(Plot şmbol: ResQual if exists or :) 
1,1,1-Trichloroethane in ABP $1 \mathrm{~A}$

Data Retrieved by AWSA v1 from GIMS on 09NOV99 at 15:50

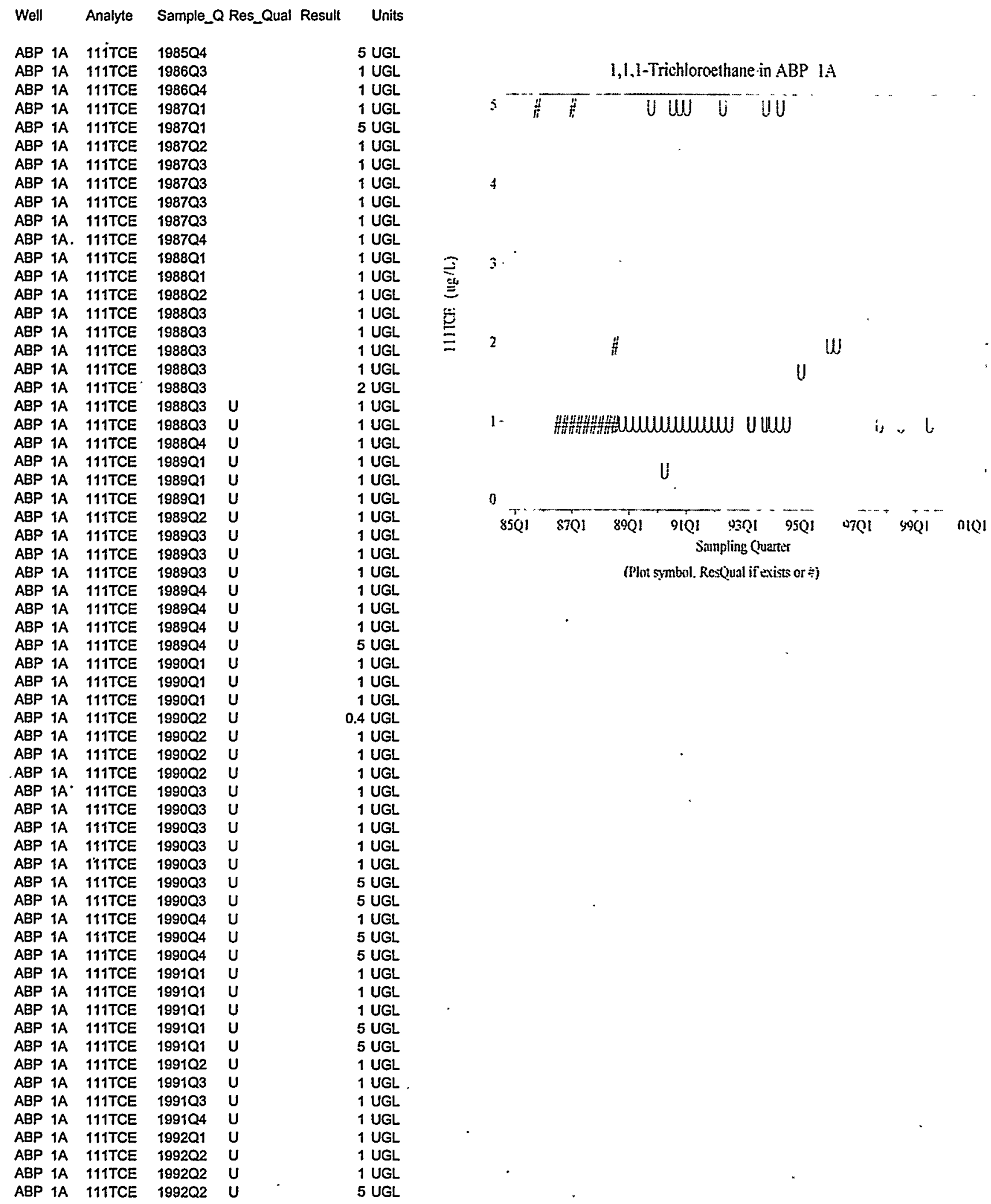


1,1,1-Trichloroethane in ABP $1 \mathrm{~A}$

Data Retrieved by AWSA v1 from GIMS on 09NOV99 at 15:50

\begin{tabular}{lllllr} 
Well & Analyte & \multicolumn{2}{c}{ Sample_Q Res_Qual Result } & Units \\
& & & & \\
ABP 1A & $111 T C E$ & 199202 & $U$ & 5 UGL \\
ABP 1A & $111 T C E$ & $1992 Q 3$ & $U$ & 1 UGL \\
ABP 1A & $111 T C E$ & $1993 Q 2$ & $U$ & 1 UGL \\
ABP 1A & $111 T C E$ & $1993 Q 3$ & UJ & 1 UGL \\
ABP 1A & $111 T C E$ & $1993 Q 4$ & $U$ & 1 UGL \\
ABP 1A & $111 T C E$ & $1993 Q 4$ & $U$ & 1 UGL \\
ABP 1A & $111 T C E$ & $1993 Q 4$ & $U$ & 5 UGL \\
ABP 1A & $111 T C E$ & $1993 Q 4$ & $U$ & 5 UGL \\
ABP 1A & $111 T C E$ & $1994 Q 1$ & $U$ & 1 UGL \\
ABP 1A & $111 T C E$ & $1994 Q 2$ & $U$ & 1 UGL \\
ABP 1A & $111 T C E$ & $1994 Q 2$ & $U$ & 1 UGL \\
ABP 1A & $111 T C E$ & $1994 Q 2$ & $U$ & 5 UGL \\
ABP 1A & $111 T C E$ & $1994 Q 2$ & $U$ & 5 UGL \\
ABP 1A & $111 T C E$ & $1994 Q 3$ & $U$ & 1 UGL \\
ABP 1A & $111 T C E$ & $1995 Q 1$ & $U$ & 1.67 UGL \\
ABP 1A & $111 T C E$ & $1996 Q 1$ & $U$ & 2 UGL \\
ABP 1A & $111 T C E$ & $1996 Q 2$ & $U$ & 2 UGL \\
ABP 1A & $111 T C E$ & $1997 Q 4$ & $U$ & 1 UGL \\
ABP 1A & $111 T C E$ & $1998 Q 3$ & $U$ & 1 UGL \\
ABP 1A & $111 T C E$ & $1998 Q 3$ & $U$ & 1 UGL \\
ABP 1A & $111 T C E$ & $1999 Q 3$ & $U$ & 1 UGL
\end{tabular}


1,1-Dichloroethylene in ABP $1 \mathrm{~A}$

Data Retrieved by AWSA v1 from GIMS on 09NOV99 at 15:50

\begin{tabular}{|c|c|c|c|c|}
\hline Well & Analyte & Sample_Q & Res_Qual f & Result \\
\hline$A B P 1 A$ & 11DCE & $1985 Q 4$ & & 5 UGL \\
\hline ABP $1 A$ & 11DCE & 1987Q1 & & 5 UGL \\
\hline ABP $1 A$ & 11DCE & 1987Q3 & & 1 UGL \\
\hline ABP $1 A$ & 11DCE & 1988Q3 & & 1 UGL \\
\hline$A B P 1 A$ & 11DCE & $1988 Q 3$ & & 2 UGL \\
\hline$A B P 1 A$ & 11DCE & $1988 Q 4$ & U & $1 \mathrm{UGL}$ \\
\hline$A B P 1 A$ & 11DCE & $1989 Q 1$ & $U$ & 1 UGL \\
\hline ABP $1 \mathrm{~A}$ & 11DCE & 1989Q1 & $\mathbf{U}$ & 1 UGL \\
\hline$A B P 1 A$ & 11DCE & $1989 Q 2$ & $U$ & 1 UGL \\
\hline ABP $1 A$ & 11DCE & 1989Q3 & $U$ & 1 UGL \\
\hline$A B P 1 A$ & 11DCE & 198903 & $U$ & 1 UGL \\
\hline$A B P 1 A$ & 11DCE & $1989 Q 3$ & $U$ & 1 UGL \\
\hline$A B P \quad 1 A$ & 11DCE & 1989Q4 & $U$ & 1 UGL \\
\hline$A B P 1 A$ & 11DCE & $1989 Q 4$ & $u$ & 1 UGL \\
\hline$A B P 1 A$ & 11DCE & $1990 Q 1$ & $U$ & $1 \mathrm{UGL}$ \\
\hline$A B P 1 A$ & 11DCE & $1990 Q 2$ & $\mathbf{u}$ & 1 UGL \\
\hline ABP $1 A$ & 11DCE & $1990 Q 2$ & $u$ & 1 UGL \\
\hline$A B P \quad 1 A$ & 11DCE & $1990 Q 3$ & $U$ & 1 UGL \\
\hline$A B P 1 A$ & 11DCE & $1990 Q 3$ & $U$ & 1 UGL \\
\hline ABP $1 A$ & 11DCE & 1990Q3 & $U$ & $1 \mathrm{UGL}$ \\
\hline$A B P 1 A$ & 11DCE & $1990 Q 3$ & $U$ & 1 UGL \\
\hline$A B P 1 A$ & 11DCE & $1990 Q 3$ & U & 1 UGL \\
\hline$A B P 1 A$ & 11DCE & $1990 Q 3$ & $u$ & 5 UGL \\
\hline$A B P 1 A$ & 11DCE & $1990 Q 3$ & $u$ & 5 UGL \\
\hline$A B P 1 A$ & 11DCE & $1990 Q 4$ & U & 1 UGL \\
\hline$A B P 1 A$ & 11DCE & $1990 Q 4$ & $u$ & 5 UGL \\
\hline$A B P 1 A$ & 11DCE & $1990 Q 4$ & U & 5 UGL \\
\hline$A B P 1 A$ & 11DCE & 1991Q1 & $U$ & 1 UGL \\
\hline$A B P \quad 1 A$ & 11DCE & 1991Q1 & $u$ & 1 UGL \\
\hline ABP $1 A$ & 11DCE & 1991Q1 & $u$ & 1 UGL \\
\hline$A B P 1 A$ & 11DCE & 1991Q1 & $u$ & 5 UGL \\
\hline ABP $1 \mathrm{~A}$ & 11DCE & 1991Q1 & $u$ & 5 UGL \\
\hline$A B P 1 A$ & 11DCE & 1991Q2 & $\mathrm{u}$ & 1 UGL \\
\hline$A B P 1 A$ & 11DCE & 1991Q3 & $u$ & 1 UGL \\
\hline ABP $1 \mathrm{~A}$ & 11DCE & 1991Q3 & $u$ & 1 UGL \\
\hline$A B P \quad 1 A$ & 11DCE & $1991 Q 4$ & $u$ & 1 UGL \\
\hline$A B P 1 A$ & 11DCE & $1992 Q 1$ & $u$ & 1 UGL \\
\hline ABP $1 A$ & 11DCE & $1992 Q 2$ & $\mathbf{u}$ & 1 UGL \\
\hline$A B P$ 1A & 11DCE & 1992Q2 & $\mathbf{u}$ & $1 \mathrm{UGL}$ \\
\hline$A B P 1 A$ & 11DCE & 199202 & $\mathbf{U}$ & 5 UGL \\
\hline ABP $1 A$ & 11DCE & $1992 Q 2$ & $u$ & 5 UGL \\
\hline$A B P$ IA & 11DCE & $1992 Q 3$ & $u$ & 1 UGL \\
\hline ABP IA & 11DCE & 1993Q2- & 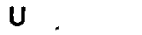 & 1 UGL \\
\hline ABP 1A & 11DCE & 1993Q3 & UJ & 1 UGL \\
\hline$A B P 1 A$ & 11DCE & 1993Q4 & & 4.1 UGL \\
\hline$A B P$ 1A & 11DCE & 1993Q4 & $u$ & 1 UGL \\
\hline ABP 1A & 11DCE & 199304 & $U$ & $5 \mathrm{UGL}$ \\
\hline ABP $1 A$ & 11DCE & $1993 Q 4$ & $u$ & 5 UGL \\
\hline$A B P 1 A$ & 110CE & 1994Q1 & $\mathrm{U}$ & 1 UGL \\
\hline ABP 1A & 11DCE & 1994Q2 & ب & $1 \mathrm{UGL}$ \\
\hline ABP $1 A$ & 11DCE & $1994 Q 2$ & $u$ & 1 UGL \\
\hline$A B P \quad 1 A$ & 11DCE & 1994Q2 & $u$ & 5 UGL \\
\hline$A B P$ A & 11DCE & 1994Q2 & $u$ & 5 UGL \\
\hline$A B P$ 1A & 11DCE & $1994 Q 3$ & $u$ & 1 UGL \\
\hline$A B P \quad 1 A$ & 11DCE & 1995Q1 & $\mathrm{u}$ & 1.67 UGL \\
\hline$A B P$ IA & 11DCE & $1896 Q 1$ & $U$ & 2 UGL \\
\hline ABP 1A & 11DCE & 1996Q2 & $U$ & 2 UGL \\
\hline ABP $1 A$ & 11DCE & $1997 Q 4$ & 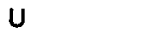 & 1 UGL \\
\hline ABP $1 A$ & 11DCE & 1998Q3 & $\mathbf{U}$ & 1 UGL \\
\hline ABP IA & 11DCE & 1998Q3 & $\mathbf{U}$ & 1 UGL \\
\hline$A B P$ 1A & 11DCE & $1999 Q 3$ & U & 1 UGL \\
\hline
\end{tabular}

I.I-Dichloroethylene in ABP IA

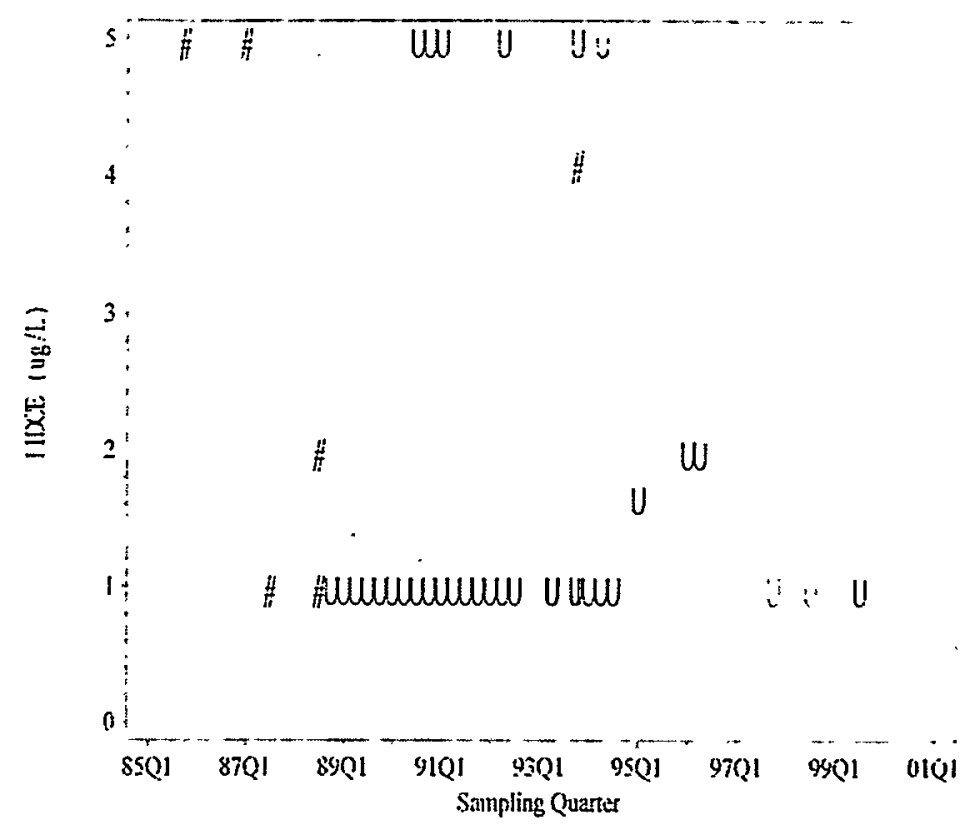

(1)hot symbol: ResQual if exists or tit) 
1,1-Dichloroethane in ABP $1 A$

Data Retrieved by AWSA v1 from GIMS on 09NOV99 at 15:50

\begin{tabular}{|c|c|c|c|c|}
\hline Well & Analyte & Sample_Q & Res_Qual & Result \\
\hline$A B P \quad 1 A$ & 11DCLE & $1985 Q 4$ & & 5 UG \\
\hline$A B P \quad 1 A$ & 11DCLE & $1887 Q 1$ & & \\
\hline$A B P \quad 1 A$ & 11DCLE & 1887Q3 & & \\
\hline$A B P \quad 1 A$ & 11DCLE & $1989 Q 3$ & $u$ & \\
\hline$A B P \quad 1 A$ & 11DCLE & $1989 Q 3$ & $U$ & \\
\hline$A B P 1 A$ & 11DCLE & $1990 Q 3$ & $u$ & \\
\hline$A B P 1 A$ & 11DCLE & $1990 Q 3$ & U & \\
\hline$A B P \quad 1 A$ & 11DCLE & 1990Q3 & u & \\
\hline$A B P 1 A$ & 11DCLE & $1990 Q 3$ & $\mathbf{U}$ & \\
\hline$A B P 1 A$ & 11DCLE & $1990 Q 3$ & $\mathbf{U}$ & \\
\hline$A B P 1 A$ & 11DCLE & $1990 Q 4$ & $\mathbf{u}$ & \\
\hline$A B P 1 A$ & 11DCLE & 199004 & $u$ & \\
\hline$A B P$ IA & 11DCLE & 1991Q1 & $u$ & \\
\hline$A B P 1 A$ & 11DCLE & 1991Q1 & $u$ & \\
\hline$A B P 1 A$ & 11DCLE & 1991Q1 & $U$ & \\
\hline$A B P \quad 1 A$ & 110CLE & 1991Q1 & $U$ & \\
\hline$A B P \quad 1 A$ & 11DCLE & 1991Q1 & $u$ & \\
\hline ABP $1 A$ & 11DCLE & 1991Q2 & $u$ & \\
\hline$A B P \quad 1 A$ & 11DCLE & $1991 Q 3$ & $u$ & \\
\hline$A B P 1 A$ & 11DCLE & 1991Q3 & $u$ & \\
\hline$A B P 1 A$ & 11DCLE & $1991 Q 4$ & $u$ & \\
\hline ABP $1 A$ & 11DCLE & $1992 Q 1$ & $u$ & \\
\hline$A B P \quad 1 A$ & 11DCLE & $1992 Q 2$ & $u$ & \\
\hline$A B P 1 A$ & 110CLE & $1992 Q 2$ & $u$ & \\
\hline$A B P 1 A$ & 11DCLE & 199202 & $u$ & \\
\hline ABP $1 A$ & 11DCLE & 199202 & $U$ & \\
\hline$A B P 1 A$ & 11DCLE & $1992 Q 3$ & $u$ & \\
\hline$A B P 1 A$ & 11DCLE & 199302 & $U$ & \\
\hline$A B P \quad 1 A$ & 11DCLE & $1993 Q 3$ & UJ & \\
\hline$A B P \quad 1 A$ & 110CLE & $1993 Q 4$ & & $12.3 \mathrm{~L}$ \\
\hline$A B P 1 A$ & 11DCLE & $1993 Q 4$ & $u$ & $1 U$ \\
\hline$A B P \quad 1 A$ & 11DCLE & $1993 Q 4$ & $U$ & \\
\hline$A B P$ 1A & 11DCLE & 1993Q4 & $U$ & \\
\hline$A B P 1 A$ & 11DCLE & 1994Q1 & $u$ & \\
\hline$A B P \quad 1 A$ & 11DCLE & 199402 & $u$ & \\
\hline$A B P \quad 1 A$ & 11DCLE & $1994 Q 2$ & $u$ & \\
\hline$A B P 1 A$ & 11DCLE & $1994 Q 2$ & $u$ & $5 u$ \\
\hline$A B P 1 A$ & 11DCLE & $1994 Q 2$ & $u$ & $5 U$ \\
\hline$A B P$ IA & 11DCLE & $1994 Q 3$ & $u$ & \\
\hline$A B P 1 A$ & 11DCLE & 1995Q1 & $U$ & 1.67 \\
\hline$A B P 1 A$ & 11DCLE & 1996Q1 & U & \\
\hline$A B P 1 A$ & 11DCLE & $1996 Q 2$ & $u$ & 2 \\
\hline$A B P 1 A$ & 11DCLE & $1997 Q 4$ & $u$ & $1 U$ \\
\hline$A B P 1 A$ & 11DCLE & $1998 Q 3$ & $u$ & \\
\hline ABP $1 A$ & 11DCLE & 1998Q3 & $u$ & \\
\hline$A B P \quad 1 A$ & 11DCLE & $1999 Q 3$ & $U$ & \\
\hline
\end{tabular}

1, 1-Dichloroethane in ABP $1 \mathrm{~A}$

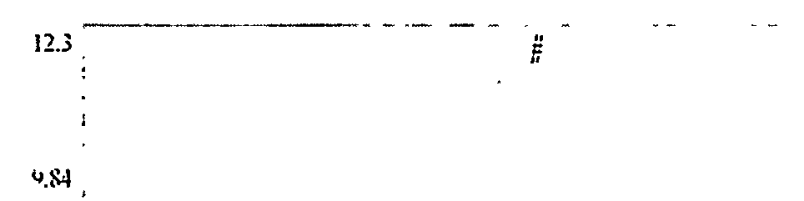

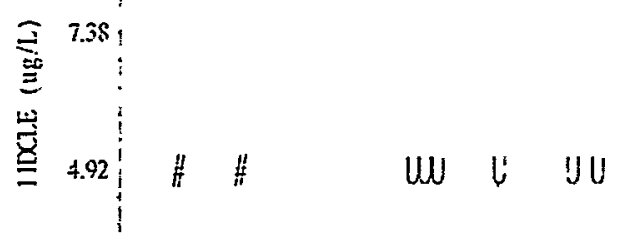

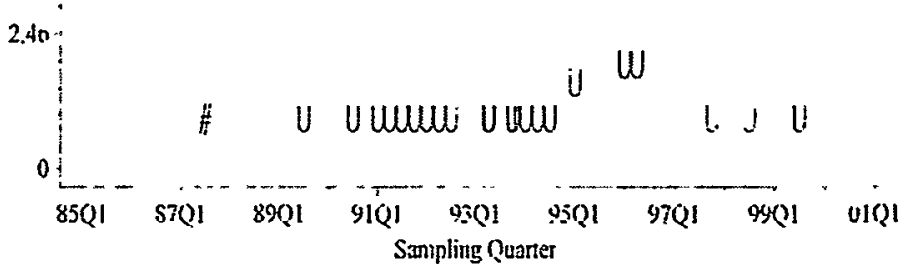

(Plot słmlul: Res(ual if exists or : ㄱ) 
Barium, total recoverable in $\mathrm{ABP} 1 \mathrm{~A}$

Data Retrieved by AWSA v1 from GIMS on 09NOV99 at 15:50

Well Analyte Sample_Q Res_Qual Result Units

ABP 1A BATOT $1994 Q 3 U$ U 3 UGL

ABP 1A BATOT 1995Q1 J 3.22 UGL

ABP 1A BATOT 1996Q1 3.16 UGL

ABP 1A BATOT 1996Q1 J 2.39 UGL

ABP 1A BATOT 1996Q1 J 2.63 UGL

ABP 1 A BATOT $199602 \mathrm{~J} \quad 2.36$ UGL

ABP 1A BATOT $199704 \mathrm{~J} \quad 3.45$ UGL

ABP 1A BATOT 1998Q3 3.68 UGL

ABP 1A BATOT $1998 Q 3 \quad 3.69$ UGL

ABP 1A BATOT 1999Q3 J 2.95 UGL

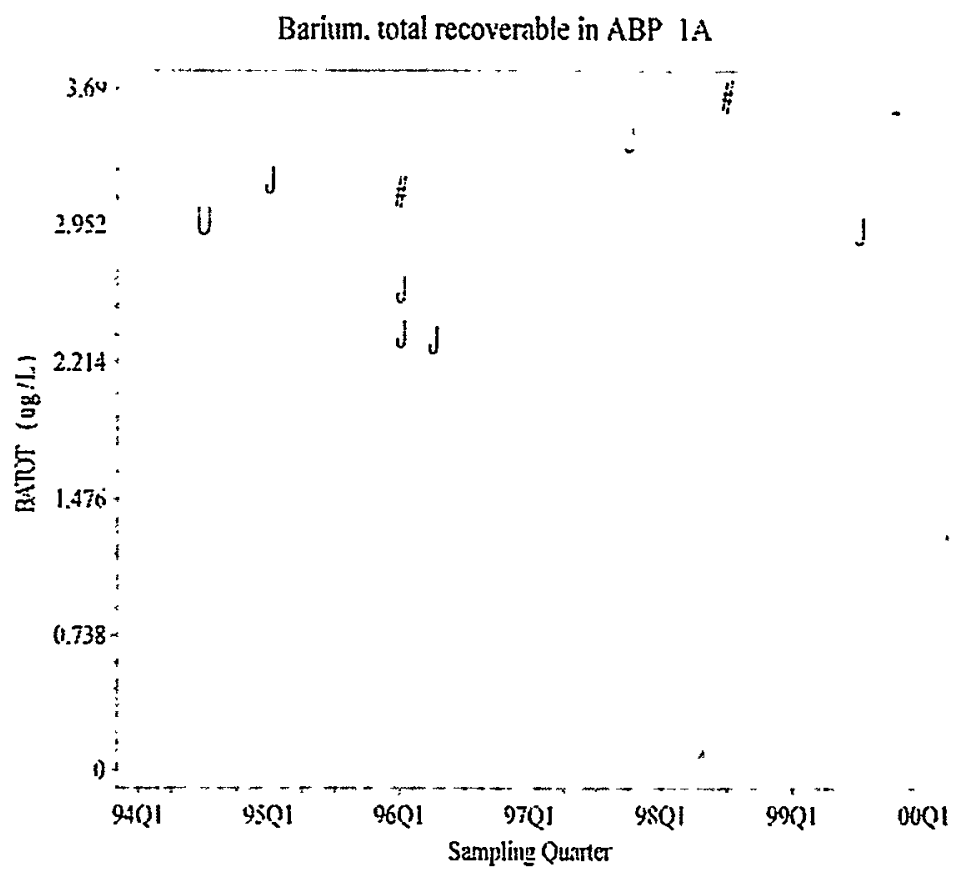

(Plot synhol: Respual if cxists ur 
Nonvolatile beta in ABP $1 A$

Data Retrieved by AWSA v1 from GIMS on 09NOV99 at 15:50

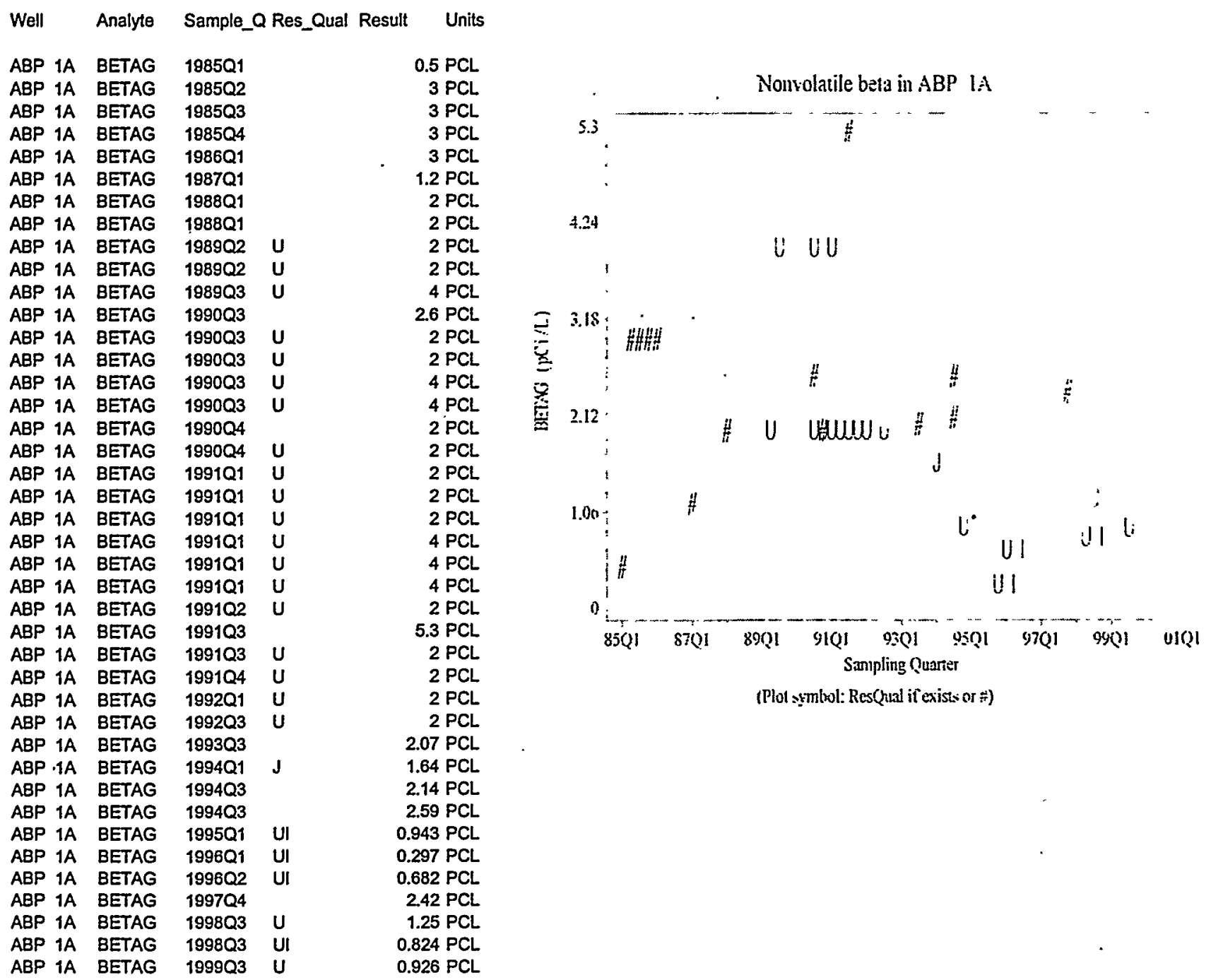


Cobalt, total recoverable in ABP $1 A$

Data Retrieved by AWSA v1 from GIMS on 09NOV99 at 15:50

\begin{tabular}{llll} 
Well & Analyte & \multicolumn{2}{c}{ Sample_Q Res_Q } \\
& & & \\
ABP 1A & COTOT & $1994 Q 3$ & U \\
ABP 1A & COTOT & $1996 Q 1$ & U \\
ABP 1A & COTOT & $1996 Q 1$ & U \\
ABP 1A & COTOT & $1996 Q 2$ & U \\
ABP 1A & COTOT & $1997 Q 4$ & U \\
ABP 1A & COTOT & $1998 Q 3$ & J \\
ABP 1A & COTOT & $1998 Q 3$ & J \\
ABP 1A & COTOT & $1999 Q 3$ & U
\end{tabular}

Units
4 UGL
0.736 UGL
4 UGL
10 UGL
5 UGL
0.12 UGL
0.182 UGL
5 UGL

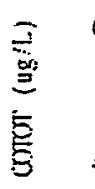

2
Cobalt, total recoverable in $A B P$ IA

s

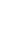

U

U

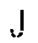

$\vdots$

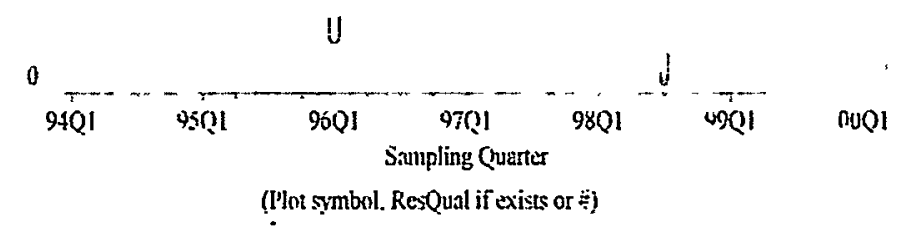


Copper, total recoverable in ABP $1 A$

Data Retrieved by AWSA v1 from GIMS on 09NOV99 at 15:50

\begin{tabular}{llllr} 
Well & Analyte & \multicolumn{2}{c}{ Sample_Q Res_Qual Result } & Units \\
& & & \\
ABP 1A & CUTOT & $1994 Q 3$ & U & 4 UGL \\
ABP 1A & CUTOT & $1996 Q 1$ & & 4.26 UGL \\
ABP 1A & CUTOT & $1996 Q 1$ & & 6.84 UGL \\
ABP 1A & CUTOT & $1996 Q 2$ & U & 25 UGL \\
ABP 1A & CUTOT & $1997 Q 4$ & & 13.2 UGL \\
ABP 1A & CUTOT & $1998 Q 3$ & & 2.32 UGL \\
ABP 1A & CUTOT & $1998 Q 3$ & U & 1.75 UGL \\
ABP 1A & CUTOT & $1999 Q 3$ & $\mathrm{~J}$ & 1.55 UGL
\end{tabular}

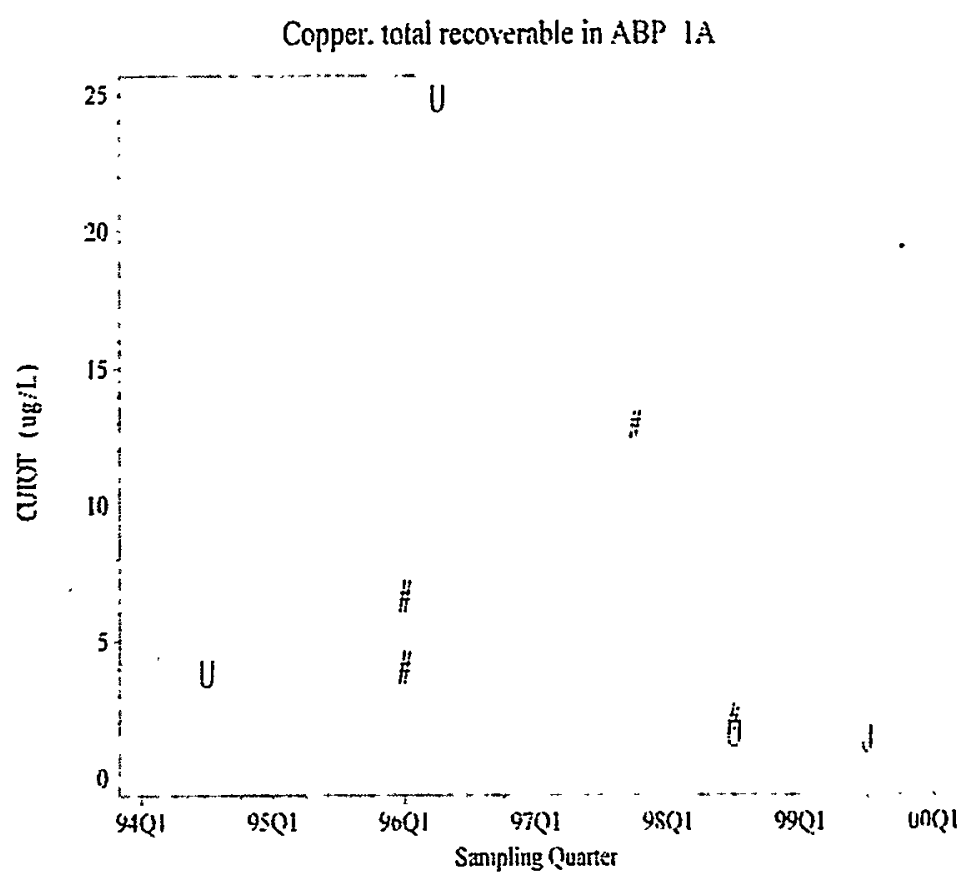

(Plot słmbol: Res(ual il exist or $\Rightarrow$ ) 
Cyanide in ABP $1 \mathrm{~A}$

Data Retrieved by AWSA v1 from GIMS on 09NOV99 at 15:50

\begin{tabular}{|c|c|c|c|c|c|}
\hline Well & Analyte & Sample_c & Res_Qual & Result & Units \\
\hline$A B P$ IA & CYN & $1990 Q 3$ & $\mathbf{u}$ & & 5 UGL \\
\hline$A B P$ IA & CYN & $1990 Q 3$ & $\mathbf{U}$ & & 5 UGL \\
\hline$A B P$ 1A & CYN & $1990 Q 3$ & U & & 5 UGL \\
\hline$A B P$ IA & CYN & $1990 Q 3$ & $U$ & & 5 UGL \\
\hline$A B P$ IA & CYN & $1990 Q 3$ & $u$ & & 5 UGL \\
\hline ABP $1 A$ & CYN & 1991Q1 & $U$ & & 5 UGL \\
\hline$A B P$ IA & CYN & 1991Q1 & U & & 5 UGL \\
\hline ABP $1 A$ & CYN & 1991Q1 & $\mathbf{U}$ & & 5 UGL \\
\hline ABP IA & CYN & 1891Q1 & $u$ & & 5 UGL \\
\hline ABP $1 A$ & CYN & 1991Q1 & $\mathbf{U}$ & & 5 UGL \\
\hline ABP $1 A$ & CYN & $1991 Q 2$ & $u$ & & 5 UGL \\
\hline$A B P$ IA & CYN & $1991 Q 2$ & $\mathbf{U}$ & & 5 UGL \\
\hline$A B P 1 A$ & CYN & $1992 Q 1$ & U & & 5 Ue \\
\hline$A B P$ IA & CYN & $1894 Q 3$ & UJ & & 5 Ue \\
\hline ABP IA & CYN & $1995 Q 1$ & $U$ & & 3.33 UGL \\
\hline ABP $1 A$ & CYN & 1996Q1 & U & & 10 UGL \\
\hline ABP $1 A$ & CYN & 199602 & $U$ & & 10 UGL \\
\hline ABP IA & CYN & $1897 Q 4$ & $u$ & & 10 UGL \\
\hline ABP $1 A$ & CYN & $1998 Q 3$ & UJ & & 10 \\
\hline ABP $1 A$ & CYN & 1898Q3 & UJ & & 10 UGL \\
\hline ABP $1 A$ & CYN & $1999 Q 3$ & $u$ & & 10 UGL \\
\hline$A B P 1 A$ & CYN & 1999Q3 & $\mathbf{u}$ & & 10 UGL \\
\hline$A B P 1 A$ & CYN & $1999 Q 3$ & $u$ & & $10 \mathrm{uc}$ \\
\hline
\end{tabular}

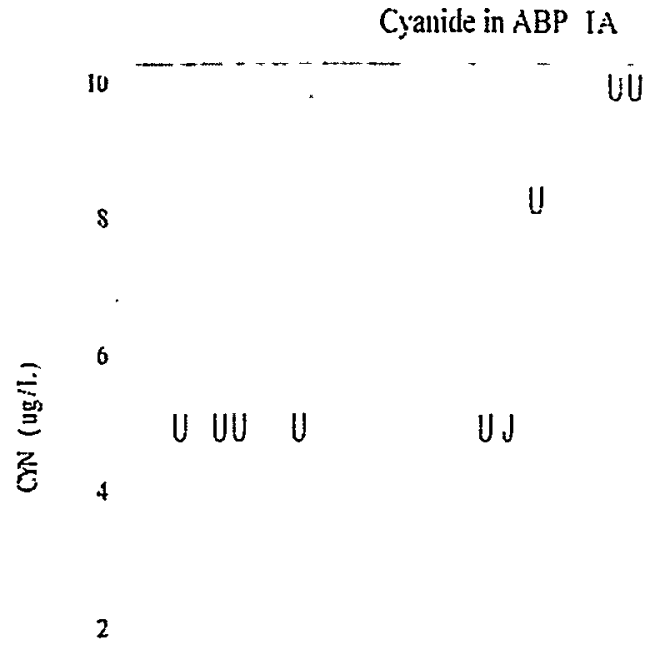


Fluoride in ABP $1 A$

Data Retrieved by AWSA v1 from GIMS on 09NOV99 at 15:50

Well Analyte Sample_Q Res_Qual Result Units

$\begin{array}{llll}\text { ABP 1A } & F & 1985 Q 1 & \\ \text { ABP 1A } & F & 1985 Q 2 & \\ \text { ABP 1A } & F & 1985 Q 3 & \\ \text { ABP 1A } & F & 1985 Q 4 & \\ \text { ABP 1A } & F & 1987 Q 1 & \\ \text { ABP 1A } & F & 1989 Q 2 & U \\ \text { ABP 1A } & F & 1989 Q 2 & U \\ \text { ABP 1A } & F & 1989 Q 3 & U \\ \text { ABP 1A } & F & 1990 Q 3 & U \\ \text { ABP 1A } & F & 1990 Q 3 & U \\ \text { ABP 1A } F & 1990 Q 3 & U \\ \text { ABP 1A } F & 1990 Q 3 & U \\ \text { ABP 1A } & F & 1990 Q 3 & U \\ \text { ABP 1A } F & 1990 Q 4 & U \\ \text { ABP 1A } F & 1990 Q 4 & U \\ \text { ABP 1A } & F & 1991 Q 1 & U \\ \text { ABP 1A } F & 1991 Q 1 & U \\ \text { ABP 1A } F & 1991 Q 1 & U \\ \text { ABP 1A } & F & 1991 Q 1 & U \\ \text { ABP 1A } F & 1991 Q 1 & U \\ \text { ABP 1A } & F & 1991 Q 2 & U \\ \text { ABP 1A } & F & 1991 Q 3 & U \\ \text { ABP 1A } F & 1991 Q 3 & U \\ \text { ABP 1A } F & 1991 Q 4 & U \\ \text { ABP 1A } & F & 1992 Q 1 & U \\ \text { ABP 1A } F & 1997 Q 4 & U \\ \text { ABP 1A } F & 1997 Q 4 & U \\ \text { ABP 1A } F & 1998 Q 3 & U \\ \text { ABP 1A } F & 1998 Q 3 & U \\ \text { ABP 1A } F & 1999 Q 3 & U \\ & & & \end{array}$

170 UGL

50 UGL 100 UGL

60 UGL

100 UGL

100 UGL

100 UGL

$250 \mathrm{UGL}$

100 UGL

$100 \mathrm{UGL}$

100 UGL

250 UGL

250 UGL

100 UGL

100 UGL

100 UGL

100 UGL

100 UGL

250 UGL

250 UGL

100 UGL

100 UGL

100 UGL

100 UGL

100 UGL

50 UGL

50 UGL

50 UGL

50 UGL

50 UGL
Fltoride in ABP IA

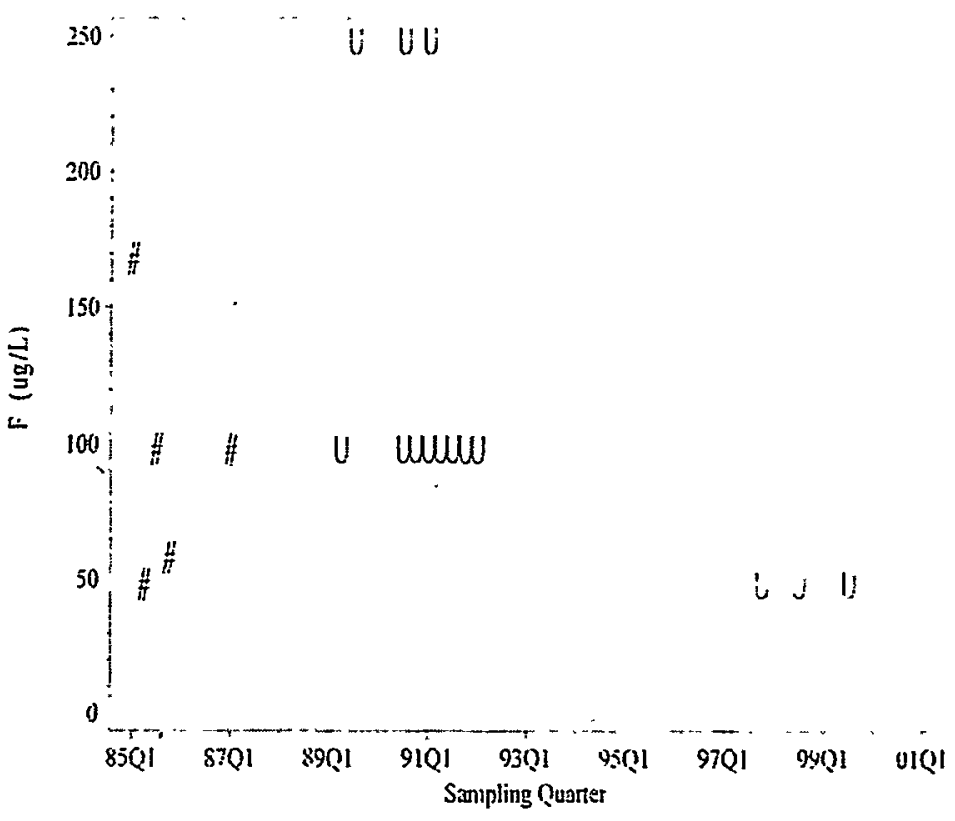

(Plot ssmbul: Res(qual if exists or : 
Mercury, total recoverable in ABP $1 A$

Data Retrieved by AWSA v1 from GIMS on 09NOV99 at 15:50

Well Analyte Sample_QRes_Qual Result Units

$\begin{array}{llll}\text { ABP 1A } & \text { HGTOT } & 1995 Q 1 & U \\ \text { ABP 1A } & \text { HGTOT } & 1996 Q 1 & J \\ \text { ABP 1A } & \text { HGTOT } & 1996 Q 2 & U J \\ \text { ABP 1A } & \text { HGTOT } & 1997 Q 4 & U \\ \text { ABP 1A } & \text { HGTOT } & 1998 Q 3 & U \\ \text { ABP 1A } & \text { HGTOT } & 1998 Q 3 & U \\ \text { ABP 1A } & \text { HGTOT } & 1999 Q 3 & U\end{array}$

0.333 UGL

0.106 UGL

0.2 UGL

0.2 UGL 0.315 UGL 0.352 UGL 0.2 UGL
Mercury, total recoverable in $A B P$ IA

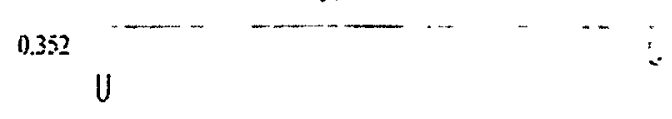

0.2816
UJ

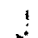

0.1408

$J$

0.0704

0

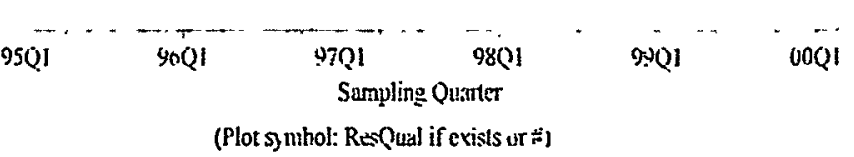


Nickel, total recoverable in ABP $1 \mathrm{~A}$

Data Retrieved by AWSA v1 from GIMS on 09NOV99 at 15:50

Well Analyte Sample_Q Res_Qua! Result Units

ABP 1A NITOT $1994 Q 3$ U 4 UGL

ABP 1A NITOT 1995Q1 U

ABP 1A NITOT 1996Q1 J

ABP 1A NITOT 1996Q1 J 3.16 UGL

ABP 1A NITOT 199601 U 25 UGL

ABP 1A NITOT 1996Q2 U 25 UGL

ABP 1A NITOT $1997 Q 4 U$ U

ABP 1A NITOT 1998Q3 U $\quad 2.38$ UGL

ABP 1A NITOT 1998Q3 U

ABP 1A NITOT $1999 Q 3 \mathrm{~J} \quad 1.4 \mathrm{UGL}$
Nickel, total recurerable in ABP $1 A$

25 (1)

20

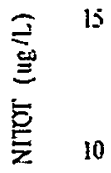

U

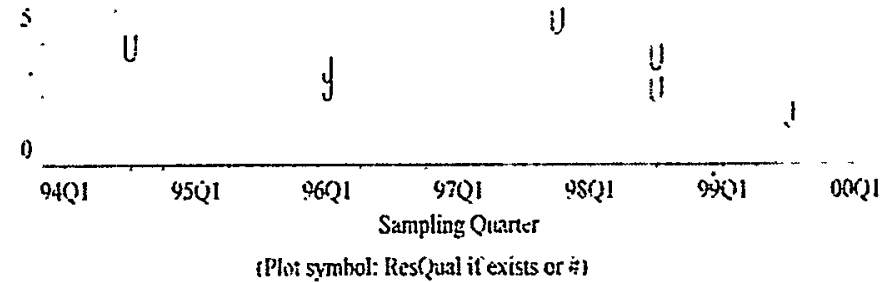


Nitrate-nitrite as nitrogen in $A B P$ $1 A$

Data Retrieved by AWSA v1 from GIMS on 09NOV99 at 15:50

Well Analyte Sample_Q Res_Qual Result Units

ABP 1A NO3NO2 $198902 \quad 560$ UGL

ABP 1A NO3NO2 198902 U

ABP 1A NO3NO2 1990Q4 830 UGL

ABP 1A NO3NO2 1990Q4 830 UGL

ABP 1A NO3NO2 1991Q1 840 UGL

ABP 1A NO3NO2 1991Q1 $850 \mathrm{UGL}$

ABP 1A NO3NO2 199101

ABP 1A NO3NO2 1991Q3 910 UGL

ABP 1A NO3NO2 1991Q3

ABP 1A NO3NO2 1991Q4 480 UGL

ABP 1A NO3NO2 1997Q4 700 UGL

ABP 1A NO3NO2 1998Q3 450 UGL

ABP 1A NO3NO2 1998Q3 460 UGL

ABP 1A NO3NO2 1999Q3 300 UGL

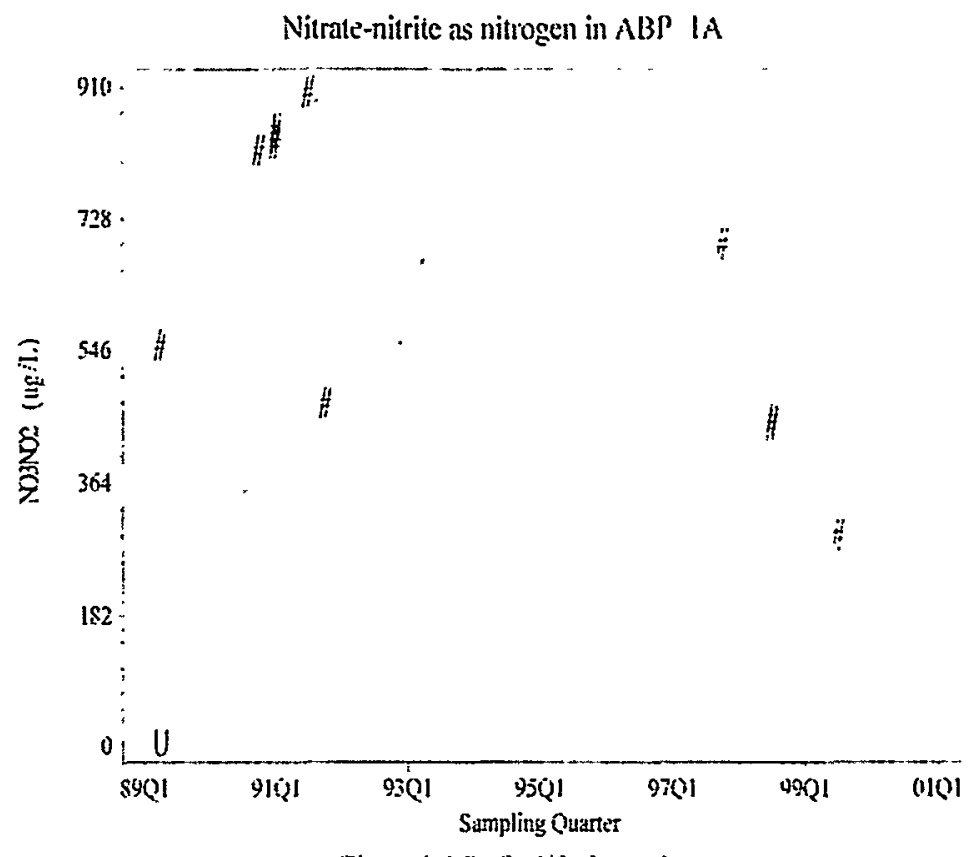

(Plot synbol: Res(pual if evists or :i) 
Selenium, total recoverable in ABP $1 \mathrm{~A}$

Data Retrieved by AWSA vi from GIMS on 09NOV99 at 15:50

Well Analyte Sample_Q Res_Qual Result Units

ABP 1A SETOT 1994Q3 U 2 UGL

ABP 1A SETOT 1995Q1 U

ABP 1A SETOT 1996Q1 U 5 UGL

ABP 1A SETOT 1996Q1 U 5 UGL

ABP IA SETOT 1996Q2 UJ 2 UGL

ABP 1A SETOT 1997Q4 U

ABP 1A SETOT $199803 \quad U$ 5 UGL

ABP 1A SETOT 1998Q3 U 5 UGL

ABP 1A SETOT $1999 Q 3 \quad U$
Selenium, tutal recoverable in ABP $1 \mathrm{~A}$

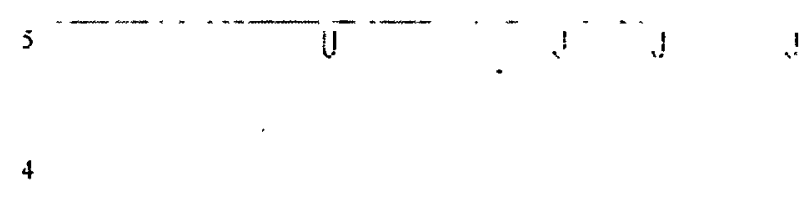

U

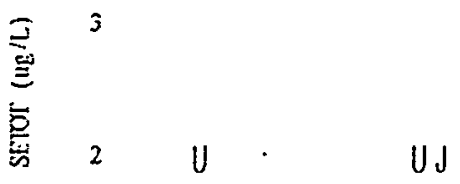

I

0

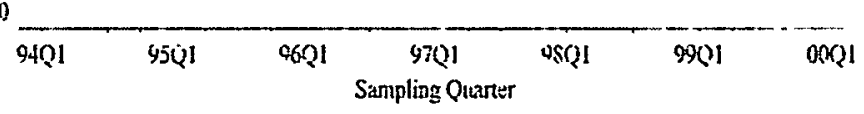

(PIot symbol: Res(gual if exists or of 
trans-1,2-Dichloroethylene in ABP $1 A$

Data Retrieved by AWSA v1 from GIMS on 09NOV99 at 15:50

\begin{tabular}{|c|c|c|c|c|}
\hline Well & Analyte & Sample_c & Res_Qual R & Result \\
\hline$A B P 1 A$ & T12DCE & $1985 Q 4$ & & 5 UG \\
\hline$A B P 1 A$ & T12DCE & 1987Q1 & & 5 UGL \\
\hline$A B P \quad 1 A$ & T12DCE & 1987Q3 & & 1 UGL \\
\hline$A B P 1 A$ & T12DCE & 1988Q3 & & 1 UGL \\
\hline$A B P 1 A$ & T12DCE & 1988Q3 & & 2 UGL \\
\hline$A B P 1 A$ & T12DCE & $1988 Q 4$ & U & 1 UGL \\
\hline$A B P 1 A$ & T12DCE & $1989 Q 1$ & U & 1 UGL \\
\hline$A B P 1 A$ & T12DCE & 1989Q1 & U & \\
\hline ABP $1 A$ & T12DCE & 1989Q2 & $u$ & 1 UGL \\
\hline ABP $1 A$ & T12DCE & 1989Q3 & $u$ & 1 UGL \\
\hline$A B P 1 A$ & T12DCE & $1989 Q 3$ & $u$ & 1 UGL \\
\hline$A B P 1 A$ & T12DCE & 198903 & $u$ & 1 UGL \\
\hline$A B P 1 A$ & T12DCE & $1989 Q 4$ & $U$ & 1 UGL \\
\hline ABP $1 A$ & T12DCE & 1989Q4 & $u$ & 1 UGL \\
\hline ABP $1 \mathrm{~A}$ & T12DCE & 1990Q1 & $u$ & 1 UGL \\
\hline $\mathrm{ABP} 1 \mathrm{~A}$ & T12DCE & $1990 Q 2$ & $U$ & 1 UGL \\
\hline$A B P$ 1A & T12DCE & 199002 & $U$ & 1 UGL \\
\hline ABP $1 \mathrm{~A}$ & T12DCE & 1990Q3 & $u$ & 1 UGL \\
\hline ABP $1 \mathrm{~A}$ & T12DCE & 199003 & $u$ & 1 UGL \\
\hline ABP $1 A$ & T12DCE & $1990 Q 3$ & $U$ & 1 UGL \\
\hline ABP $1 A$ & T12DCE & $1990 Q 3$ & $\mathbf{U}$ & 1 UGL \\
\hline ABP $1 \mathrm{~A}$ & T12DCE & 1990Q3 & $\mathbf{u}$ & 1 UGL \\
\hline ABP $1 \mathrm{~A}$ & T120CE & $1990 Q 4$ & $u$ & $1 \mathrm{UGL}$ \\
\hline ABP $1 \mathrm{~A}$ & T12DCE & 199004 & $u$ & 5 UGL \\
\hline$A B P 1 A$ & T12DCE & $1990 Q 4$ & $u$ & 5 UGL \\
\hline$A B P 1 A$ & T12DCE & 1991Q1 & $U$ & 1 UGL \\
\hline$A B P 1 A$ & T12DCE & 1991Q1 & $u$ & 1 UGL \\
\hline ABP $1 \mathrm{~A}$ & T12DCE & 1991Q1 & U & 1 UGL \\
\hline$A B P$ 1A & T12DCE & 1991Q2 & U & 1 UGL \\
\hline ABP $1 \mathrm{~A}$ & T120CE & 1991Q3 & U & 1 UGL \\
\hline$A B P 1 A$ & T12DCE & 1991Q3 & $U$ & 1 UGL \\
\hline$A B P 1 A$ & T12DCE & $1991 Q 4$ & U & 1 UGL \\
\hline ABP $1 \mathrm{~A}$ & T12DCE & 1992Q1 & $U$ & \\
\hline ABP $1 \mathrm{~A}$ & T12DCE & 1992Q2 & $u$ & JGL \\
\hline $\mathrm{ABP} \quad 1 \mathrm{~A}$ & T12DCE & 199202 & $U$ & 1 UGL \\
\hline$A B P$ 1A & T120CE & $1992 Q 2$ & $U$ & 5 UGL \\
\hline$A B P 1 A$ & T12DCE & 199202 & $u$ & 5 UGL \\
\hline ABP $1 \mathrm{~A}$ & T12DCE & $1992 Q 3$ & $u$ & 1 UGL \\
\hline ABP $1 A$ & T12DCE & 199302 & $u$ & \\
\hline ABP $1 A$ & T12DCE & 1993Q3 & UJ & \\
\hline ABP $1 \mathrm{~A}$ & T12DCE & $1993 Q 4$ & $\mathbf{U}$ & 1 UGL \\
\hline$A B P \quad 1 A$ & T12DCE & $1993 Q 4$ & U & 1 UGL \\
\hline$A B P$ 1A & T12DCE & $1993 Q 4$ & $u$ & 5 UGL \\
\hline ABP $1 \mathrm{~A}$ & T120CE & $1993 Q 4$ & $u$ & 5 UGL \\
\hline ABP $1 \mathrm{~A}$ & T12DCE & 1994Q1 & $u$ & 1 UGL \\
\hline $\mathrm{ABP} 1 \mathrm{~A}$ & T12DCE & 1994Q2 & $U$ & 1 UGL \\
\hline ABP $1 A$ & T12DCE & 1994Q2 & $\mathbf{U}$ & 1 UGL \\
\hline$A B P 1 A$ & T12DCE & $1994 Q 2$ & $\mathbf{U}$ & 5 UGL \\
\hline$A B P \quad 1 A$ & T12DCE & 1994Q2 & $U$ & 5 UGL \\
\hline ABP $1 A$ & T12DCE & 1894Q3 & $u$ & 1 UGL \\
\hline ABP $1 A$ & T12DCE & 1995Q1 & $\mathbf{U}$ & $1.67 \mathrm{UGL}$ \\
\hline ABP $1 A$ & T12DCE & 1997Q4 & U & 1 UGL \\
\hline$A B P$ IA & T12DCE & 1998Q3 & 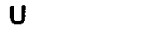 & 1 UGL \\
\hline$A B P 1 A$ & T12DCE & $1998 Q 3$ & $U$ & 1 UGL \\
\hline$A B P$ & T12DCE & 1999Q3 & U & 1 UGL \\
\hline
\end{tabular}

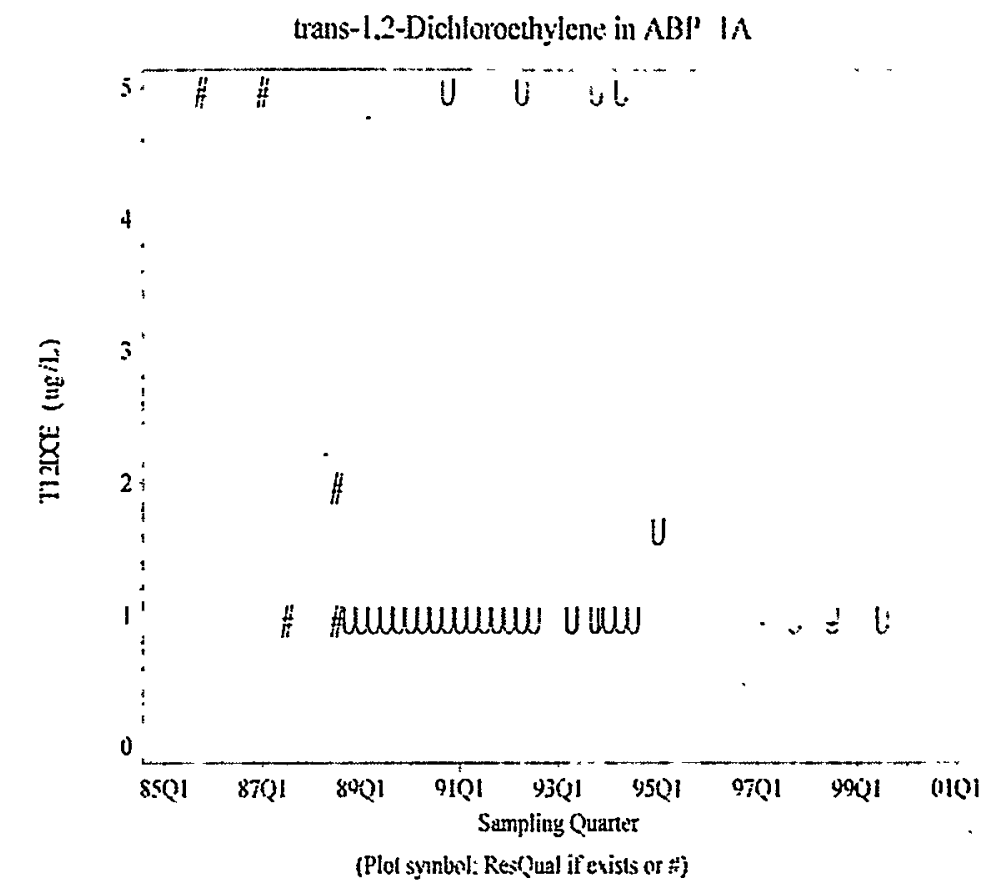


Data Retrieved by AWSA v1 from GIMS on 09NOV99 at 15:50

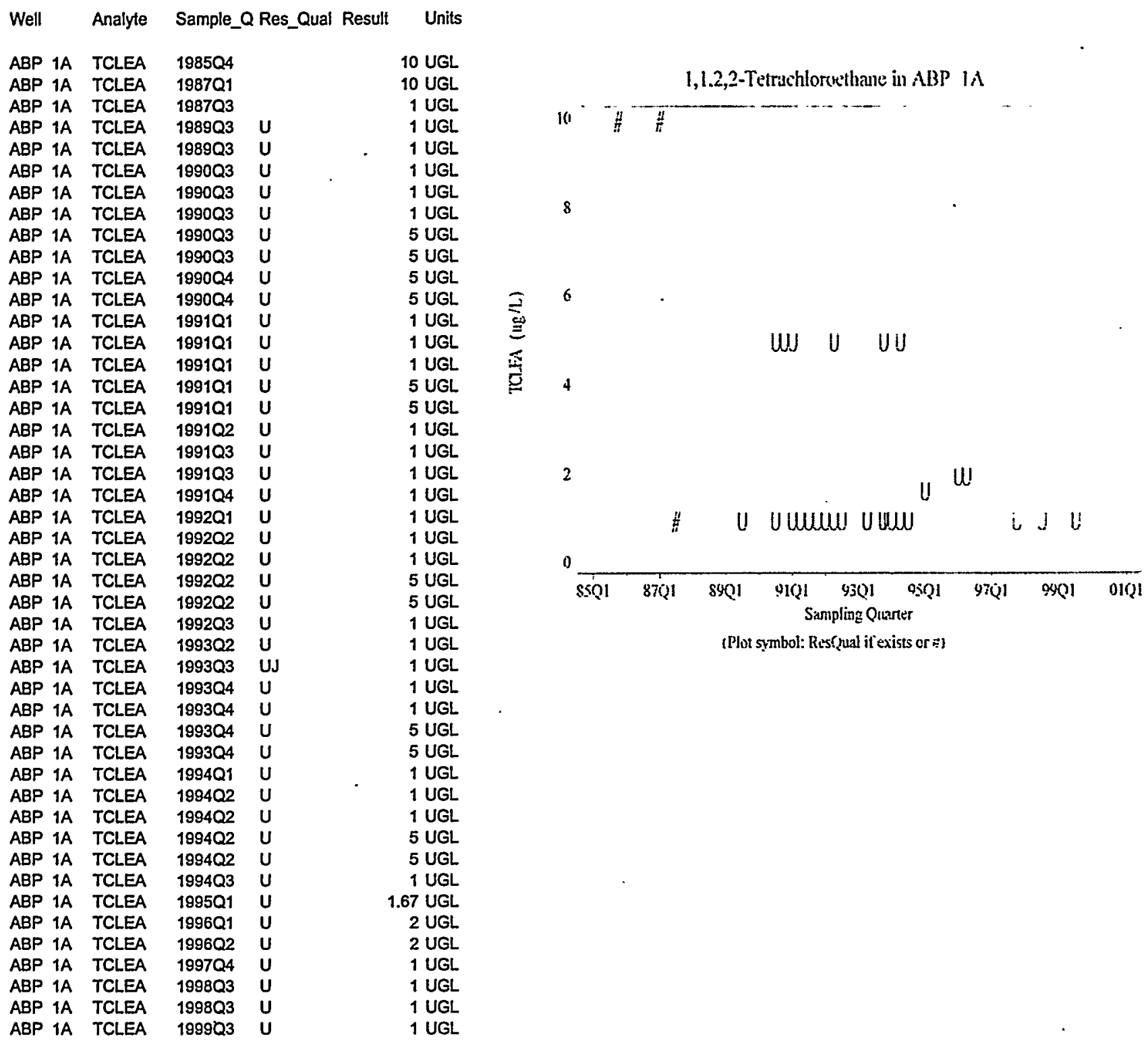


Tetrachloroethylene in ABP $1 \mathrm{~A}$

Data Retrieved by AWSA v1 from GIMS on 09NOV99 at 15:50

\begin{tabular}{|c|c|c|c|c|c|}
\hline Well & & Analyte & Sample_C & Res_Qual & Result \\
\hline ABP & $1 A$ & TCLEE & $1985 Q 4$ & & 5 UGL \\
\hline ABP & $1 A$ & TCLEE & $1986 Q 3$ & & 3.72 UGL \\
\hline ABP & 1A & TCLEE & $1986 Q 4$ & & 3.37 UGL \\
\hline$A B P$ & $1 A$ & TCLEE & 1987Q1 & & 1 UGL \\
\hline ABP & $1 A$ & TCLEE & $1987 Q 1$ & & 5 UGL \\
\hline ABP & $1 A$ & TCLEE & 1987Q2 & & 1 UGL \\
\hline ABP & $1 A$ & TCLEE & 1987Q3 & & 1 UGL \\
\hline ABP & $1 A$ & TCLEE & $1987 Q 3$ & & 1 UGL \\
\hline ABP & $1 A$ & TCLEE & $1987 Q 3$ & & 1 UGL \\
\hline ABP & $1 A$ & TCLEE & 1987Q3 & & 1.34 UGL \\
\hline ABP & $1 \mathrm{~A}$ & TCLEE & $1987 Q 4$ & & 1 UGL \\
\hline ABP & $1 A$ & TCLEE & 1988Q1 & & 1 UGL \\
\hline ABP & $1 A$ & TCLEE & $1988 Q 1$ & & 1 UGL \\
\hline ABP & $1 A$ & TCLEE & $1988 Q 2$ & & 1 UGL \\
\hline ABP & $1 A$ & TCLEE & $1988 Q 3$ & & 1 UGL \\
\hline ABP & $1 A$ & TCLEE & 1988Q3 & & 1 UGL \\
\hline ABP & $1 \mathrm{~A}$ & TCLEE & $1988 Q 3$ & & 1 UGL \\
\hline ABP & $1 \mathrm{~A}$ & TCLEE & $1988 Q 3$ & & 1 UGL \\
\hline ABP & $1 A$ & TCLEE & $1988 Q 3$ & & 2 UGL \\
\hline ABP & $1 A$ & TCLEE & 1988Q3 & & 3 UGL \\
\hline ABP & $1 A$ & TCLEE & $1988 Q 3$ & U & 1 UGL \\
\hline ABP & $1 A$ & TCLEE & $1988 Q 4$ & $U$ & 1 UGL \\
\hline ABP & $1 A$ & TCLEE & 1989Q1 & & 1.64 UGL \\
\hline$A B P$ & $1 A$ & TCLEE & 1989Q1 & $U$ & $1 \mathrm{UGL}$ \\
\hline ABP & $1 A$ & TCLEE & 1989Q1 & $U$ & 1 UGL \\
\hline ABP & $1 A$ & TCLEE & $1989 Q 2$ & $U$ & 1 UGL \\
\hline$A B P$ & $1 A$ & TCLEE & 1989Q3 & $U$ & 1 UGL \\
\hline ABP & $1 A$ & TCLEE & $1989 Q 3$ & $U$ & 1 UGL \\
\hline ABP & $1 A$ & TCLEE & 1989Q3 & $u$ & 1 UGL \\
\hline ABP & $1 A$ & TCLEE & 1989Q4 & $u$ & 1 UGL \\
\hline ABP & $1 A$ & TCLEE & $1989 Q 4$ & $\mathbf{U}$ & 1 UGL \\
\hline ABP & $1 A$ & TCLEE & $1989 Q 4$ & $u$ & 1 UGL \\
\hline ABP & $1 A$ & TCLEE & $1989 Q 4$ & $u$ & 5 UGL \\
\hline ABP & $1 A$ & TCLEE & $1990 Q 1$ & $u$ & 1 UGL \\
\hline ABP & $1 A$ & TCLEE & 1990Q1 & $u$ & 1 UGL \\
\hline ABP & $1 A$ & TCLEE & 1990Q1 & U & 1 UGL \\
\hline ABP & $1 A$ & TCLEE & 199002 & $\mathbf{u}$ & 0.4 UGL \\
\hline ABP & $1 A$ & TCLEE & $1990 Q 2$ & $u$ & 1 UGL \\
\hline ABP & $1 A$ & TCLEE & 199002 & $u$ & 1 UGL \\
\hline ABP & $1 A$ & TCLEE & $1990 Q 2$ & $u$ & 1 UGL \\
\hline ABP & $1 A$ & TCLEE & 199003 & & $1 \mathrm{UGL}$ \\
\hline ABP & $1 A$ & TCLEE & 199003 & $u$ & 1 UGL \\
\hline $\mathrm{ABP}$ & $1 A$ & TCLEE & $1990 Q 3$ & $U$ & 1 UGL \\
\hline$A B P$ & $1 A$ & TCLEE & $1990 Q 3$ & $U$ & 1 UGL \\
\hline$A B P$ & $1 A$ & TCLEE & 199003 & $U$ & 1 UGL \\
\hline ABP & $1 A$ & TCLEE & 199003 & $u$ & 10 UGL \\
\hline ABP & $1 A$ & TCLEE & $1990 Q 3$ & u & 10 UGL \\
\hline ABP & $1 A$ & TCLEE & $1990 Q 4$ & U & 1 UGL \\
\hline ABP & $1 A$ & TCLEE & $1990 Q 4$ & u & 5 UGL \\
\hline ABP & $1 A$ & TCLEE & $1990 Q 4$ & $u$ & 5 UGL \\
\hline ABP & $1 A$ & TCLEE & 1991Q1 & $u$ & 1 UGL \\
\hline ABP & $1 \mathrm{~A}$ & TCLEE & 1991Q1 & $u$ & 1 UGL \\
\hline ABP & $1 \mathrm{~A}$ & TCLEE & 1991Q1 & $u$ & 1 UGL \\
\hline ABP & $1 A$ & TCLEE & $1891 Q 1$ & U & 10 UGL \\
\hline$A B P$ & $1 A$ & TCLEE & 199101 & $u$ & 10 UGL \\
\hline$A B P$ & $1 \mathrm{~A}$ & TCLEE & 199102 & U & 1 UGL \\
\hline $\mathrm{ABP}$ & $1 A$ & TCLEE & 1991Q3 & $\mathbf{u}$ & $1 \mathrm{UGL}$ \\
\hline ABP & $1 \mathrm{~A}$ & TCLEE & 199103 & U & 1 UGL \\
\hline$A B P$ & $1 A$ & TCLEE & 1991Q4 & $U$ & 1 UGL \\
\hline$A B P$ & $1 \mathrm{~A}$ & TCLEE & $1992 Q 1$ & $u$ & 1 UGL \\
\hline ABP & $1 \mathrm{~A}$ & TCLEE & $1992 Q 2$ & & 2.77 UGL \\
\hline ABP & $1 A$ & TCLEE & 1992Q2 & & 6.23 UGL \\
\hline & & TCLEE & $1992 Q 2$ & J & 1.97 UGL \\
\hline
\end{tabular}

Tetrachloroethylent in ABP $\mid A$

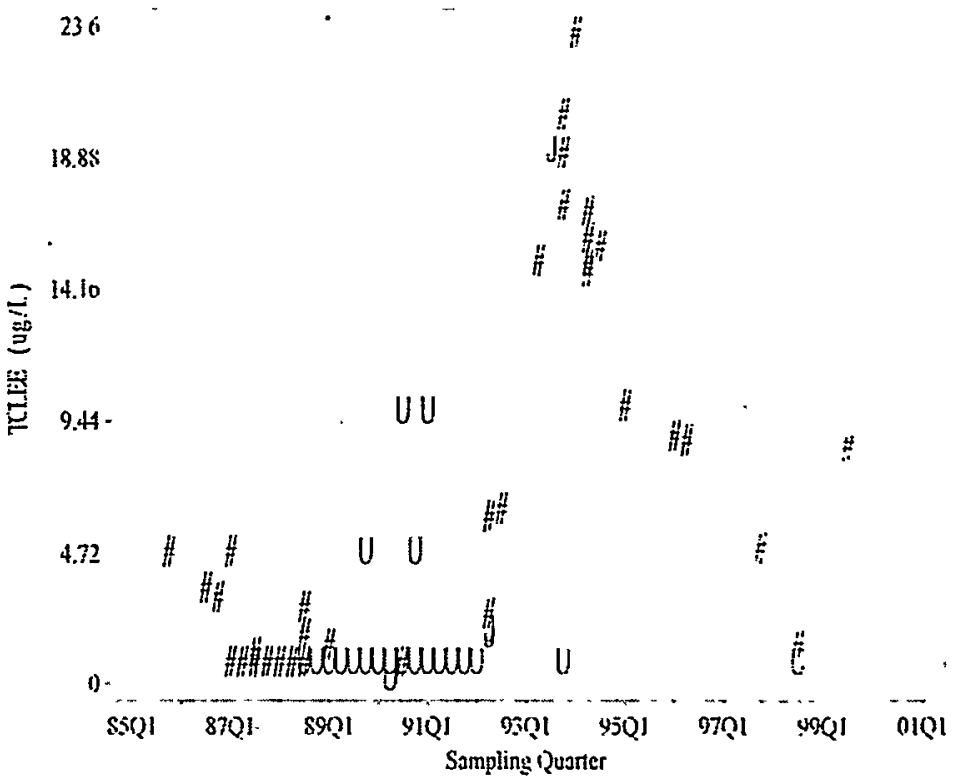

(Plot syninol: ResQual if exists or iै) 
Zinc, total recoverable in ABP $1 A$

Data Retrieved by AWSA v1 from GIMS on 23NOV99 at 08:45

Well Analyte Sample_Q Res_Qual Result Units

ABP 1A ZNTOT $1994 Q 3 \quad 20.1$ UGL

ABP IA ZNTOT 1995Q1 U 3.33 UGL

ABP 1A ZNTOT 1996Q1 J 3.12 UGL

ABP 1A ZNTOT 1996Q1 UJ 5 UGL

ABP 1A ZNTOT 199602 U

ABP 1A ZNTOT 1997Q4 45.9 UGL

ABP 1A ZNTOT $199803 \quad U \quad 5$ UGL

ABP 1A ZNTOT 1998Q3 U

ABP 1A ZNTOT $1999 Q 3 \mathrm{~J} \quad 3.23 \mathrm{UGL}$
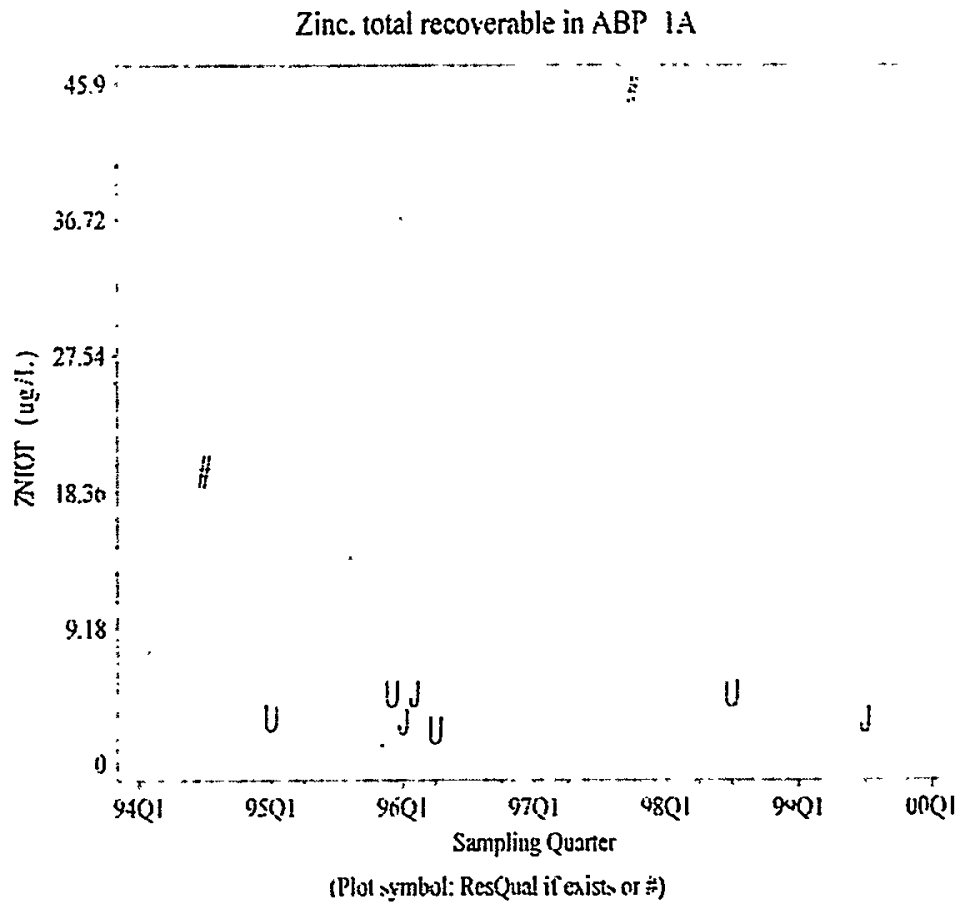
WELL ABP-4 DATA 
Field $\mathrm{pH} \quad$ in ABP 4

Dala Retrieved by AWSA v1 from GIMS on 09NOV99 at 16:22

Well Analyte Sample_Q Res_Qual Result Units

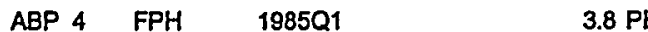

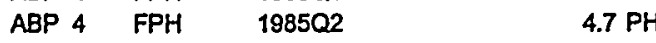

ABP 4 FPH $198503 \quad 4.4 \mathrm{PH}$

$\begin{array}{llll}\text { ABP } 4 & \text { FPH } & 1985 Q 4 & 4.3 \mathrm{PH}\end{array}$

ABP 4 FPH $198601 \quad 4.1 \mathrm{PH}$

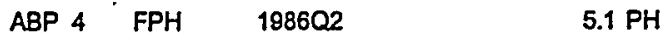

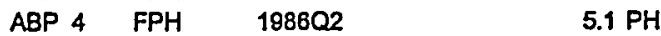

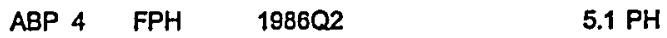

ABP 4 FPH 198603

ABP 4 FPH $1986 Q 44.5 \mathrm{PH}$

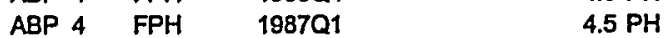

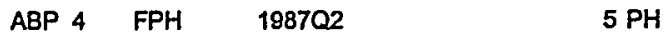

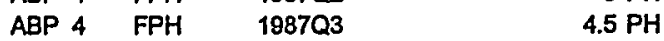

$\begin{array}{lll}\text { ABP } 4 & \text { FPH } & 1987 Q 4\end{array}$

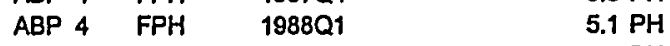

ABP 4 FPH 1988Q1

ABP 4 FPH 1988Q1

ABP 4 FPH $1988 Q 2$

ABP 4 FPH 1988 2

ABP 4 FPH 198802

ABP 4 FPH 1988Q3

ABP 4 FPH 1988Q4

ABP 4 FPH 1989Q1

ABP 4 FPH 198902

ABP 4 FPH 1989Q3

ABP 4 FPH 1989 Q3

ABP 4 FPH 1989 3 3

ABP 4 FPH 198904

ABP 4 FPH 1990 Q1

ABP 4 FPH 1990Q1

ABP 4 FPH 199002

ABP 4 FPH $1990 \mathrm{~B} 3$

ABP 4 FPH $1990 Q 4$

ABP 4 FPH 1990Q4

ABP 4 . FPH 1991Q1

ABP 4 FPH 199102

ABP $4 \quad \mathrm{FPH}, 1991 \mathrm{Q} 3$

ABP 4 FPH 1991Q4

ABP $4 \quad F P H \quad 1992 Q 1$

ABP 4 FPH $1992 Q 1$

ABP 4 FPH 199202

ABP $4 \quad \mathrm{FPH} \quad 1892 \mathrm{Q} 2$

ABP 4 FPH $1992 \mathrm{Q}$

ABP 4. FPH 1992Q4

ABP 4 FPH 1993Q1

ABP 4 FPH 199302

$\begin{array}{lll}A B P & \mathrm{FPH} & 1993 \mathrm{Q} 3\end{array}$

ABP 4 FPH 1993Q4

ABP 4 FPH 1994Q1

ABP 4 FPH 1994Q2

ABP 4 FPH $1994 Q 3$

ABP 4 FPH 1995Q1

ABP 4 FPH 199601

ABP 4 FPH 199602

ABP 4 FPH 199704

ABP 4 FPH 1998Q3

ABP 4 FPH 1999 Q3

ABP 4 FPH $1999 Q 3$
Field pH in ABP 4

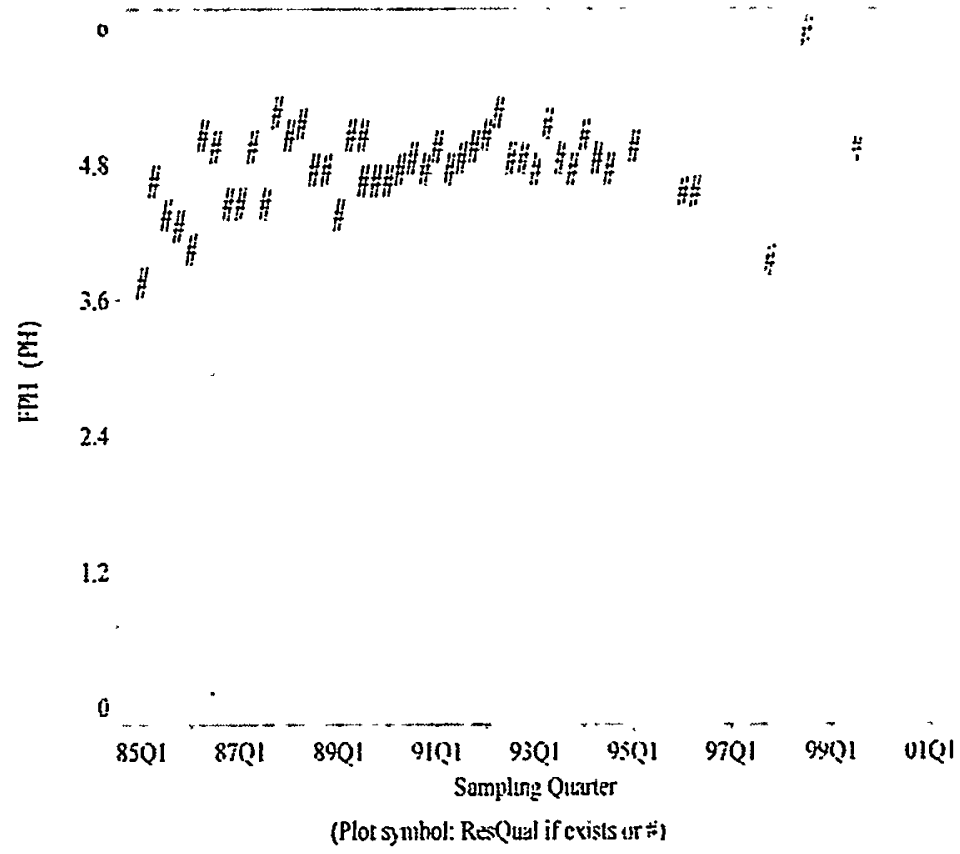


Specific Conductance in ABP 4

Data Retrieved by AWSA v1 from GIMS on 09NOV99 at 16:22

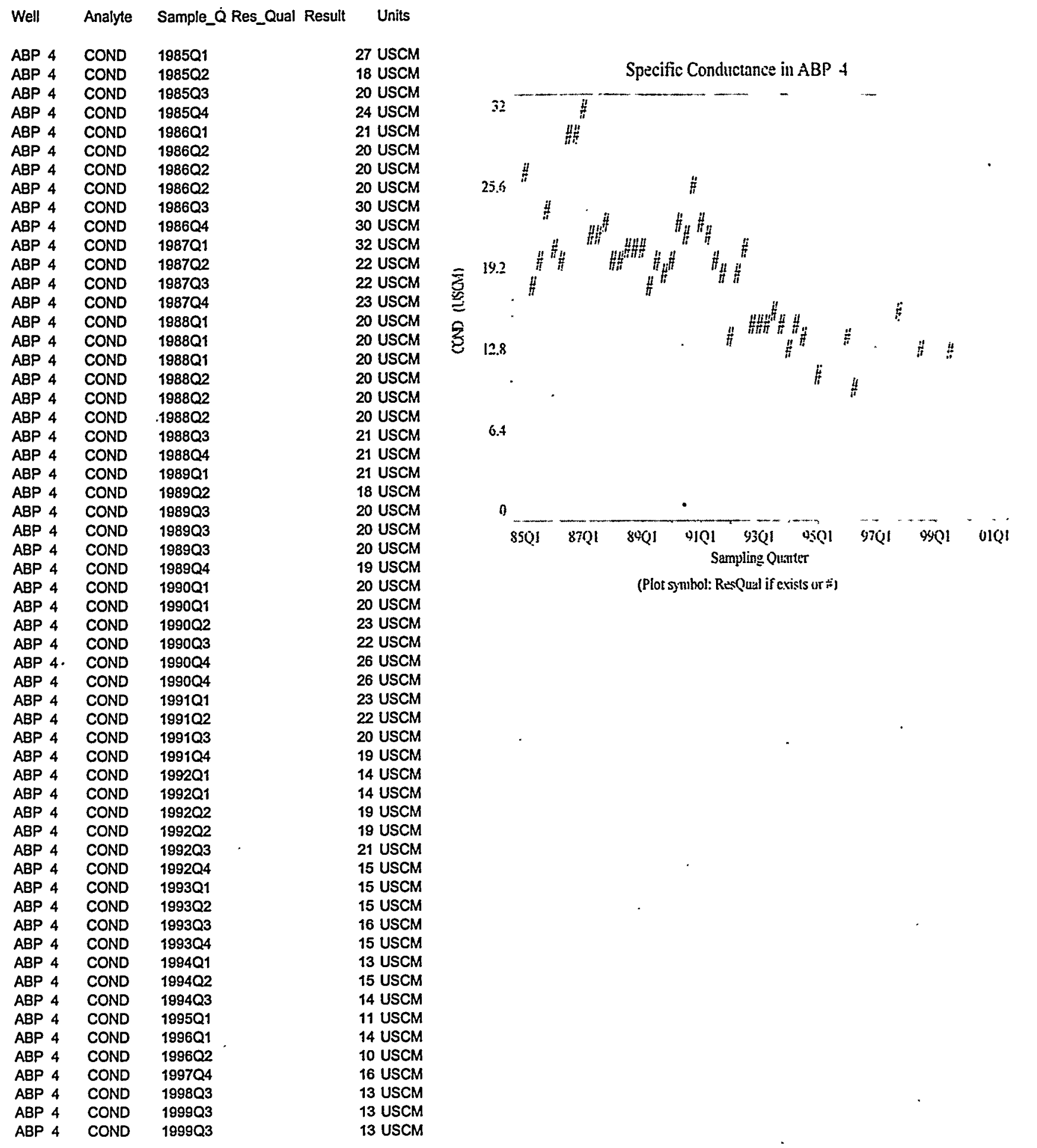


Calcium, total recoverable in ABP 4

Data Retrieved by AWSA v1 from GIMS on 09NOV99 at 15:50

\begin{tabular}{|c|c|c|}
\hline Well & Analyte & Sample_ \\
\hline ABP 4 & САTOT & 1993Q1 \\
\hline$A B P 4$ & CATOT & 199301 \\
\hline ABP 4 & CATOT & $1993 Q 1$ \\
\hline ABP 4 & САТОТ & 1993Q1 \\
\hline ABP 4 & CATOT & 199301 \\
\hline $\mathrm{ABP} 4$ & СATOT & 1996Q1 \\
\hline $\mathrm{ABP} 4$ & CATOT & $1996 Q 1$ \\
\hline ABP 4 & CATOT & $1996 Q 1$ \\
\hline ABP 4 & САTOT & 199601 \\
\hline ABP 4 & CATOT & 1996Q1 \\
\hline$A B P 4$ & САTOT & $1996 Q 2$ \\
\hline ABP 4 & САTOT & $1997 Q 4$ \\
\hline$A B P 4$ & CATOT & 1998Q3 \\
\hline ABP 4 & CATOT & 1998Q3 \\
\hline $\mathrm{ABP} 4$ & САTOT & 1999Q3 \\
\hline ABP 4 & САTOT & 1999Q3 \\
\hline
\end{tabular}

Units

540 UGL 599 UGL 615 UGL 634 UGL 637 UGL 503 UGL 560 UGL 399 UGL 425 UGL 426 UGL 557 UGL 477 UGL 543 UGL 645 UGL $470 \mathrm{UGL}$ 477 UGL

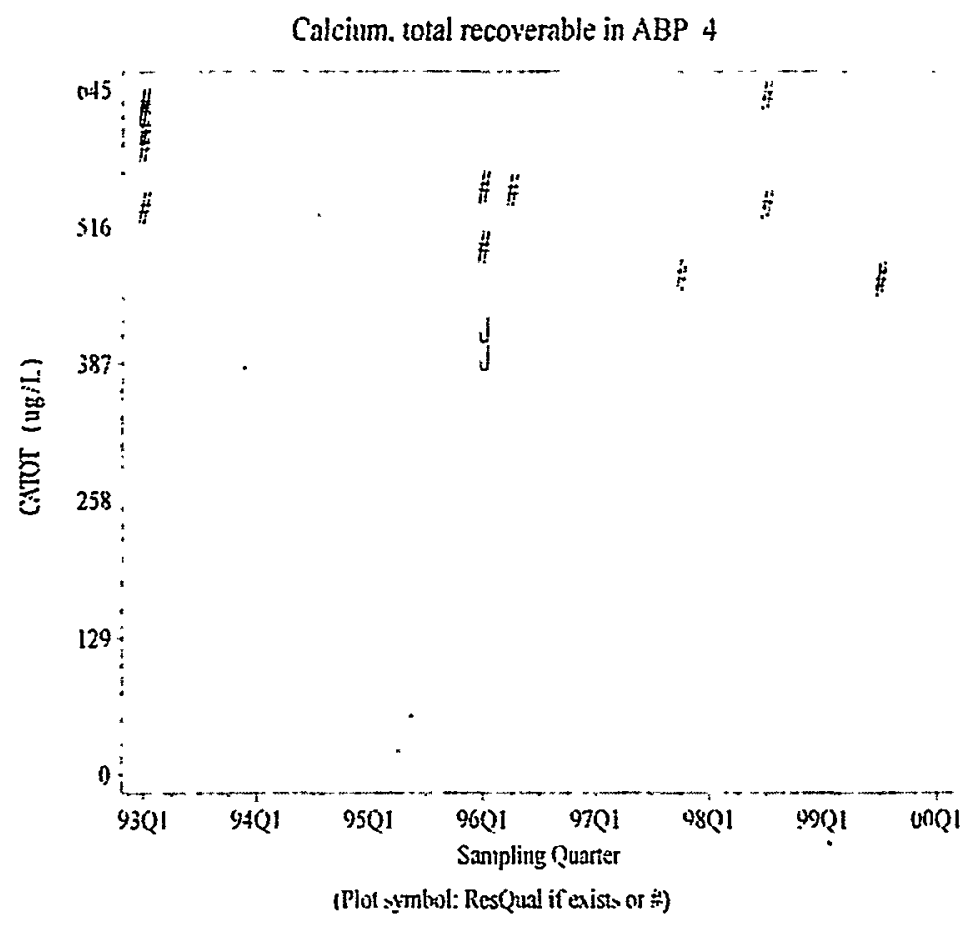


Potassium, total recoverable in ABP 4

Data Retrieved by AWSA v1 from GIMS on 09NOV99 at 15:50

Well Analyte Sample_Q Res_Qual Result Units

$\begin{array}{lllll}\text { ABP 4 } & \text { KTOT } & 1993 Q 1 & J & 519 \text { UGL } \\ \text { ABP 4 } & \text { KTOT } & 1993 Q 1 & U & 500 \text { UGL } \\ \text { ABP 4 } & \text { KTOT } & 1993 Q 1 & U & 500 \text { UGL } \\ \text { ABP 4 } & \text { KTOT } & 1993 Q 1 & U & 500 \text { UGL } \\ \text { ABP 4 } & \text { KTOT } & 1993 Q 1 & U & 500 \text { UGL } \\ \text { ABP 4 } & \text { KTOT } & 1996 Q 1 & & 148 \text { UGL } \\ \text { ABP 4 } & \text { KTOT } & 1996 Q 1 & & 171 \text { UGL } \\ \text { ABP 4 } & \text { KTOT } & 1996 Q 1 & & 188 \text { UGL } \\ \text { ABP 4 } & \text { KTOT } & 1996 Q 1 & J & 189 \text { UGL } \\ \text { ABP 4 } & \text { KTOT } & 1996 Q 1 & J & 192 \text { UGL } \\ \text { ABP 4 } & \text { KTOT } & 1996 Q 2 & U & 2000 \text { UGL } \\ \text { ABP 4 } & \text { KTOT } & 1997 Q 4 & & 177 \text { UGL } \\ \text { ABP 4 } & \text { KTOT } & 1998 Q 3 & 193 \text { UGL } \\ \text { ABP 4 } & \text { KTOT } & 1998 Q 3 & 196 \text { UGL } \\ \text { ABP 4 } & \text { KTOT } & 1999 Q 3 & 168 \text { UGL } \\ \text { ABP 4 } & \text { KTOT } & 1999 Q 3 & 176 \text { UGL }\end{array}$

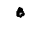

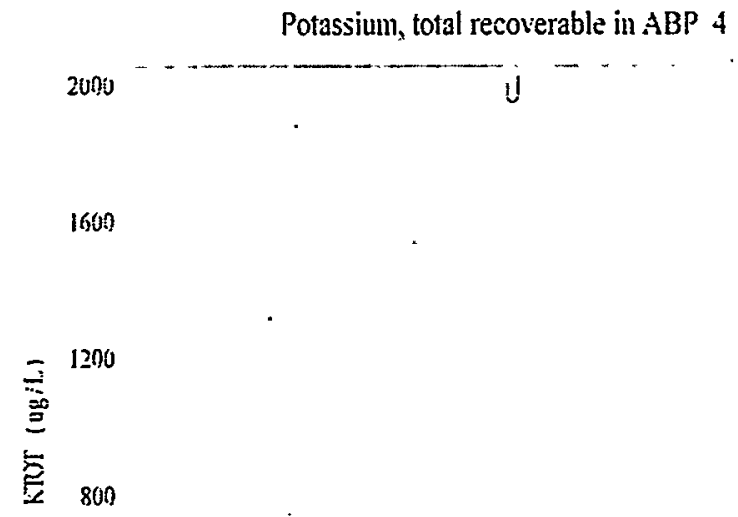

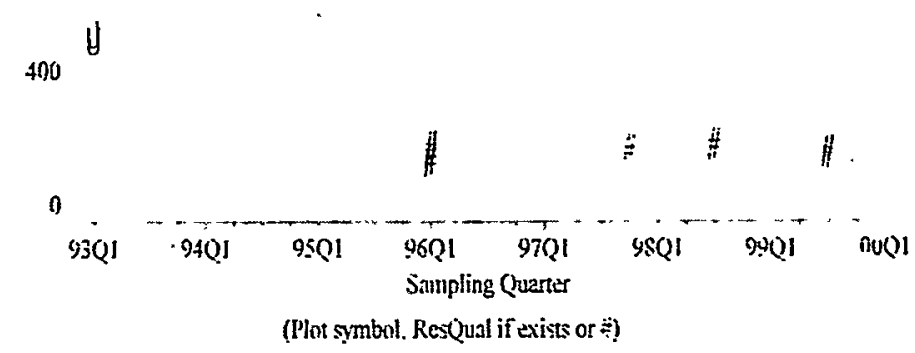


Sodium, total recoverable in ABP 4

Data Retrieved by AWSA v1 from GIMS on 09NOV99 at 15:50

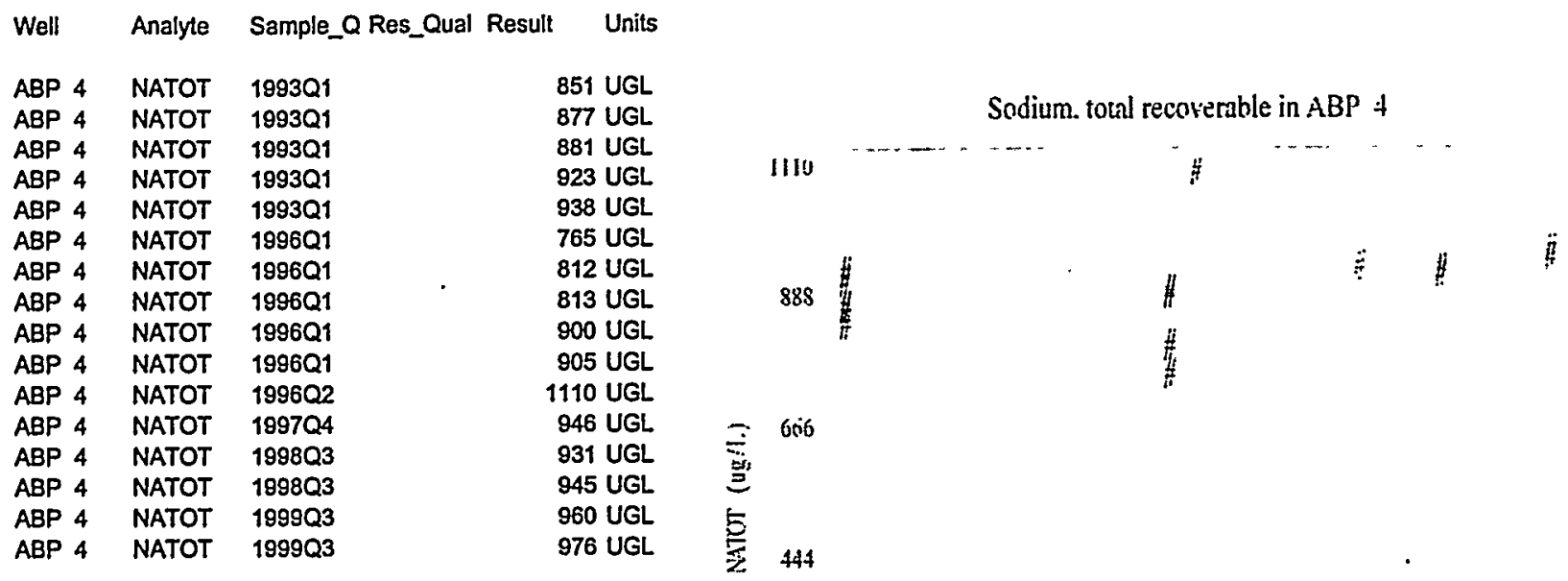

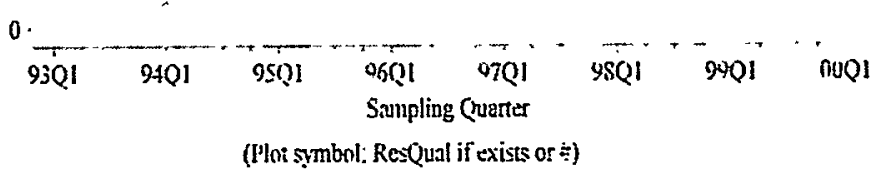


Chromium, total recoverable in ABP 4

Data Retrieved by AWSA v1 from GIMS on 09NOV99 at 15:50

$\begin{array}{llll}\text { Well } & \text { Analyte } & \text { Sample_Q Res } \\ & & & \\ \text { ABP 4 } & \text { CRTOT } & 1993 Q 1 & \mathrm{U} \\ \text { ABP 4 } & \text { CRTOT } & 1993 Q 1 & \mathrm{U} \\ \text { ABP 4 } & \text { CRTOT } & 1993 Q 1 & \mathrm{U} \\ \text { ABP 4 } & \text { CRTOT } & 1993 Q 1 & \mathrm{U} \\ \text { ABP 4 } & \text { CRTOT } & 1993 Q 1 & \mathrm{U} \\ \text { ABP 4 } & \text { CRTOT } & 1995 Q 1 & \mathrm{U} \\ \text { ABP 4 } & \text { CRTOT } & 1996 Q 1 & \mathrm{~J} \\ \text { ABP 4 } & \text { CRTOT } & 1996 Q 1 & \mathrm{U} \\ \text { ABP 4 } & \text { CRTOT } & 1996 Q 1 & \mathrm{U} \\ \text { ABP 4 } & \text { CRTOT } & 1996 Q 1 & \mathrm{U} \\ \text { ABP 4 } & \text { CRTOT } & 1996 Q 1 & \mathrm{U} \\ \text { ABP 4 } & \text { CRTOT } & 1996 Q 2 & \mathrm{U} \\ \text { ABP 4 } & \text { CRTOT } & 1997 Q 4 & \mathrm{U} \\ \text { ABP 4 } & \text { CRTOT } & 1998 Q 3 & \mathrm{~J} \\ \text { ABP 4 } & \text { CRTOT } & 1999 Q 3 & \mathrm{~J} \\ \text { ABP 4 } & \text { CRTOT } & 1999 Q 3 & \mathrm{U}\end{array}$

Units

4 UGL 4 UGL 4 UGL 4 UGL 4 UGL 6.67 UGL 0.655 UGL 4 UGL 7 UGL 7 UGL

7 UGL 25 UGL 5 UGL 1.43 UGL 3.1 UGL 5 UGL
Chromiun. total recoverable in ABP 4

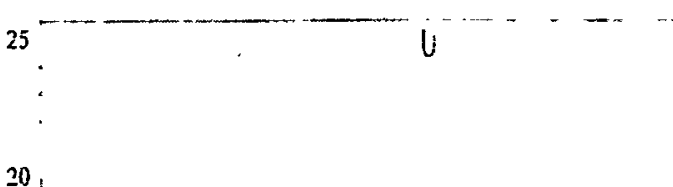

20

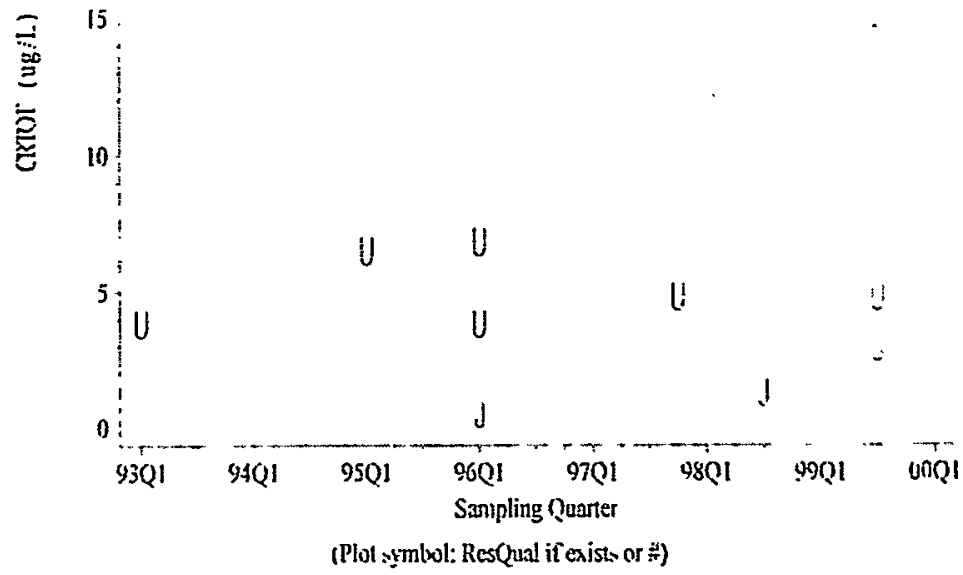


Iron, total recoverable in ABP 4

Data Retrieved by AWSA v1 from GIMS on 09NOV99 at 15:50

\begin{tabular}{|c|c|c|c|c|}
\hline Well & Analyte & Sample_C & I Res_Qual & Result \\
\hline BPP 4 & FETOT & 1993Q1 & $J$ & 6.3 UGL \\
\hline ABP 4 & FETOT & 1993Q1 & $J$ & 11.2 UGL \\
\hline$A B P 4$ & FETOT & 1993Q1 & $u$ & 4 UC \\
\hline ABP 4 & FETOT & 1993Q1 & $u$ & 4 UGL \\
\hline ABP 4 & FETOT & 1993Q1 & $u$ & 4 UGL \\
\hline ABP 4 & FETOT & 1996Q1 & & $186 \mathrm{U}$ \\
\hline ABP 4 & FETOT & 1996Q1 & $J$ & $16.8 \cup G$ \\
\hline ABP 4 & FETOT & 1996Q1 & $J$ & 17.4 UE \\
\hline ABP 4 & FETOT & $1996 Q 1$ & $J$ & 19.2 UG \\
\hline ABP 4 & FETOT & 1996Q1 & UJ & 18 UE \\
\hline ABP 4 & FETOT & $1996 \mathrm{Q} 2$ & $U$ & 100 UGL \\
\hline ABP 4 & FETOT & 1998̣Q3 & & 130. UGL \\
\hline$A B P 4$ & FETOT & $1999 Q 3$ & & 50.2 UGL \\
\hline & FETOT & $1999 Q 3$ & - & 61.4 UG \\
\hline
\end{tabular}

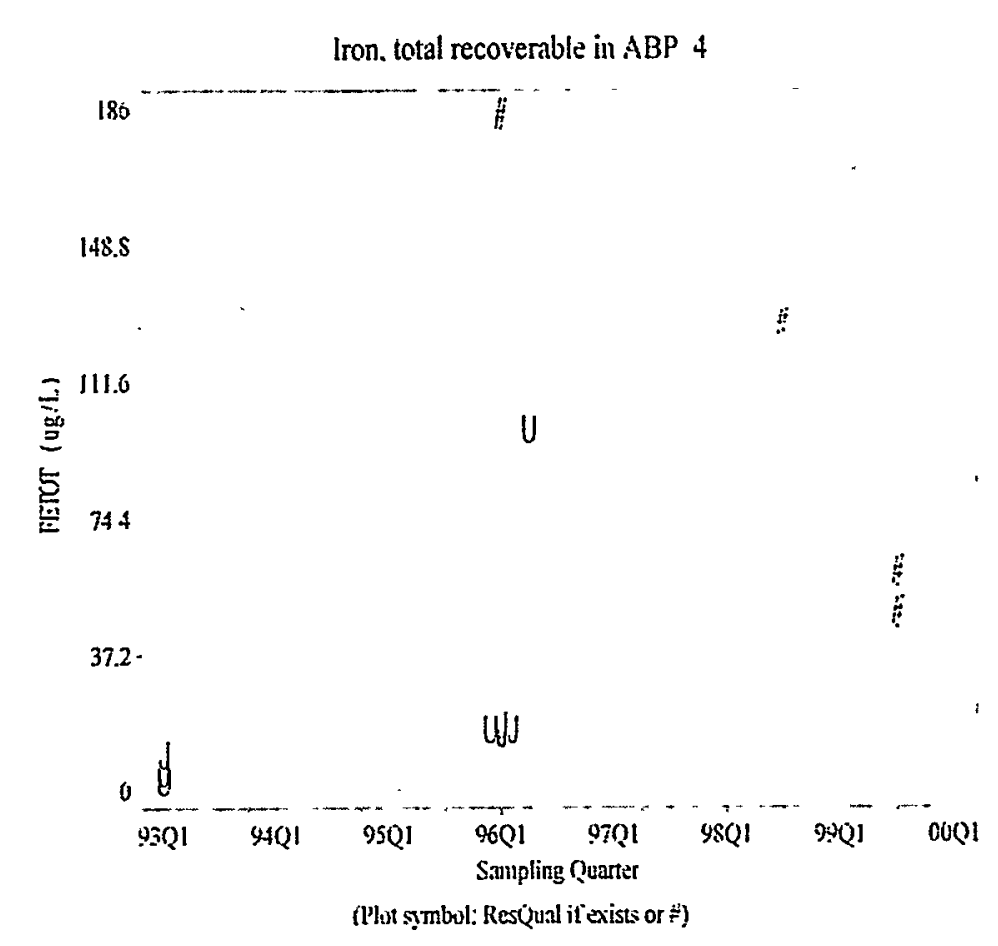


Manganese, total recoverable in ABP 4

Data Retrieved by AWSA v1 from GIMS on 09NOV99 at 15:50

\begin{tabular}{|c|c|c|c|c|c|}
\hline Well & & Analyte & Sample_ & Res_Qual & Result \\
\hline ABP & 4 & MNTOT & 1993Q1 & & $2.5 \mathrm{UGL}$ \\
\hline ABP & 4 & MNTOT & 1993Q1 & & $2.6 \mathrm{UGL}$ \\
\hline ABP & 4 & MNTOT & 1993Q1 & & 2.8 UGL \\
\hline$A B P$ & 4 & MNTOT & 1993Q1 & & 3.12 UGL \\
\hline$A B P$ & 4 & MNTOT & 1993Q1 & & 3.18 UGL \\
\hline ABP & 4 & MNTOT & 1993Q3 & & $2.47 \mathrm{UGL}$ \\
\hline ABP & 4 & MNTOT & 1994Q1 & $\mathbf{J}$ & $2.46 \mathrm{UGL}$ \\
\hline ABP & 4 & MNTOT & 1994Q1 & $\mathbf{J}$ & $2.47 \mathrm{UGL}$ \\
\hline ABP & 4 & MNTOT & 1994Q1 & $\mathbf{J}$ & 2.49 UGL \\
\hline ABP & 4 & MNTOT & $1994 Q 1$ & $\mathbf{J}$ & 2.53 UGL \\
\hline$A B P$ & 4 & MNTOT & 1994Q3 & $\mathbf{J}$ & 2.33 UGL \\
\hline ABP & 4 & MNTOT & 1995Q1 & $\mathbf{J}$ & 2.32 UGL \\
\hline ABP & 4 & MNTOT & 1996Q1 & & 2.09 UGL \\
\hline ABP & 4 & MNTOT & 1996Q1 & & 2.15 UGL \\
\hline ABP & 4 & MNTOT & 1996Q1 & $\mathbf{J}$ & 2.5 UGL \\
\hline ABP & 4 & MNTOT & 1996Q1 & $\mathbf{J}$ & $2.5 \mathrm{UGL}$ \\
\hline$A B P$ & 4 & MNTOT & 1996Q1 & $\mathbf{J}$ & 3.7 UGL \\
\hline$A B P$ & 4 & MNTOT & 1996Q2 & $\mathbf{U}$ & 10 UGL \\
\hline$A B P$ & 4 & MNTOT & 1997Q4 & $\mathbf{J}$ & 4.41 UGL \\
\hline $\mathrm{ABP}$ & 4 & MNTOT & $1998 Q 3$ & & 4.01 UGL \\
\hline$A B P$ & 4 & MNTOT & 1999Q3 & $\mathbf{U}$ & $10 \mathrm{UGL}$ \\
\hline $\mathrm{ABP}$ & 4 & MNTOT & 1999Q3 & $\mathbf{U}$ & 10 UGL \\
\hline
\end{tabular}

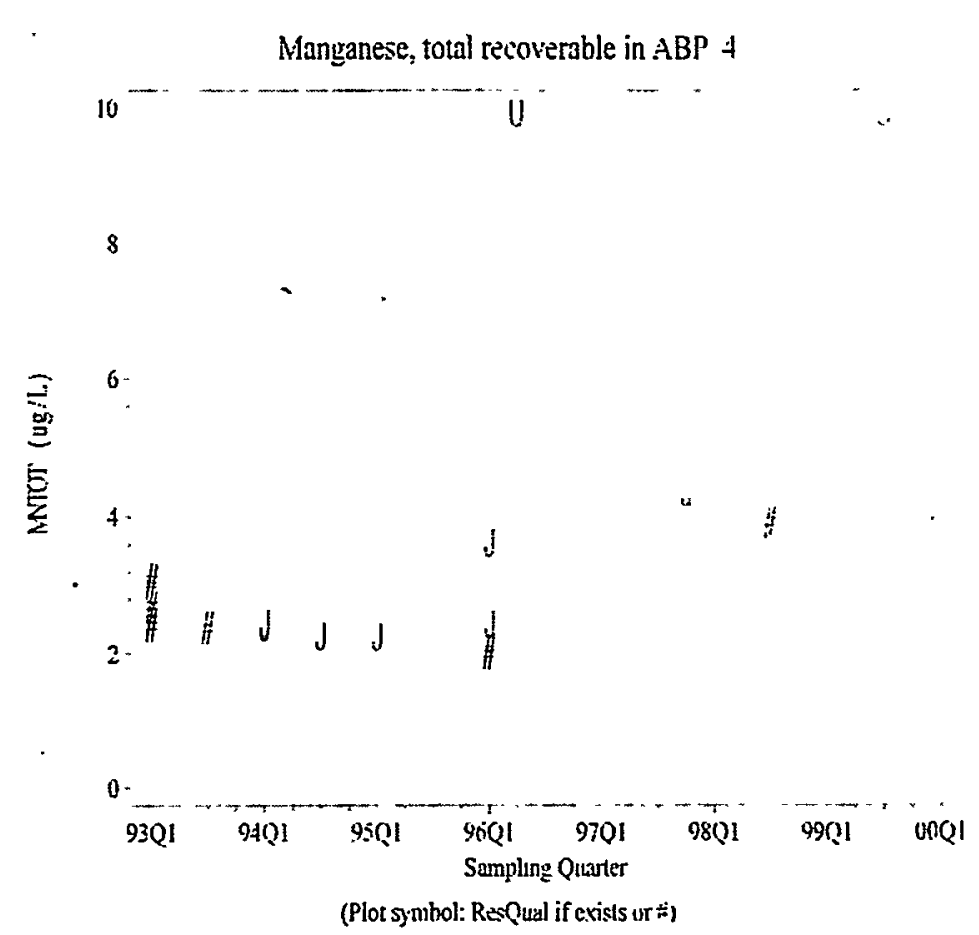


Aluminum, total recoverable in ABP 4

Data Retrieved by AWSA v1 from GIMS on 09NOV99 at 15:50

\begin{tabular}{|c|c|c|c|c|c|}
\hline Well & Analyte & Sample_ & Res_Qual & Result & Units \\
\hline $\mathrm{ABP}$ & ALTOT & $1992 Q 4$ & $\mathbf{U}$ & & $20 \mathrm{UGL}$ \\
\hline$A B P$ & ALTOT & 1993Q1 & $U$ & & $20 \mathrm{UGL}$ \\
\hline$A B P$ & ALTOT & 1993Q1 & $\mathbf{U}$ & & $20 \mathrm{UGL}$ \\
\hline ABP & ALTOT & 199301 & $\mathbf{U}$ & & 20 UGL \\
\hline$A B P$ & ALTOT & 1993Q1 & $\mathbf{U}$ & & $20 \mathrm{UGL}$ \\
\hline ABP & ALTOT & 199301 & $\mathbf{U}$ & & $20 \mathrm{UGL}$ \\
\hline ABP & ALTOT & $1993 Q 3$ & $\mathbf{U}$ & & 20 UGL \\
\hline ABP & ALTOT & 1994Q1 & $\mathbf{U}$ & & $20 \mathrm{UGL}$ \\
\hline ABP & ALTOT & 1994Q1 & $\mathbf{U}$ & & $20 \mathrm{UGL}$ \\
\hline $\mathrm{ABP}$ & ALTOT & 1994Q1 & $\mathbf{U}$ & & $20 \mathrm{UGL}$ \\
\hline$A B P$ & ALTOT & 1994Q1 & $\mathbf{U}$ & & $20 \mathrm{UGL}$ \\
\hline ABP & ALTOT & 1994Q3 & $\mathbf{U}$ & & $20 \mathrm{UGL}$ \\
\hline$A B P$ & ALTOT & 1995Q1 & $\mathbf{U}$ & & 33.3 UGL \\
\hline$A B P$ & ALTOT & 1996Q1 & $\mathbf{U}$ & & 14.8 UGL \\
\hline$A B P$ & ALTOT & 1996Q1 & $\mathbf{U}$ & & 21.1 UGL \\
\hline ABP & ALTOOT & 1996Q1 & $\mathbf{U}$ & & 146 UGL \\
\hline ABP & ALTOT & 1996Q1 & $\mathbf{U}$ & & 146 UGL \\
\hline ABP & ALTOT & 1996Q1 & $\mathbf{U}$ & & 146 UGL \\
\hline$A B P$ & ALTOT & 1996Q2 & $\mathbf{U}$ & & 250 UGL \\
\hline $\mathrm{ABP}$ & ALTOT & 1997Q4 & $U$ & & 50 UGL \\
\hline$A B P$ & ALTOT & 1998Q3 & $J$ & & 13.1 UGL \\
\hline$A B P$ & ALTOT & 1999Q3 & $\mathbf{U}$ & & 50 UGL \\
\hline $\mathrm{ABP}$ & ALTOT & 199903 & $\mathbf{U}$ & & 50 UGL \\
\hline
\end{tabular}

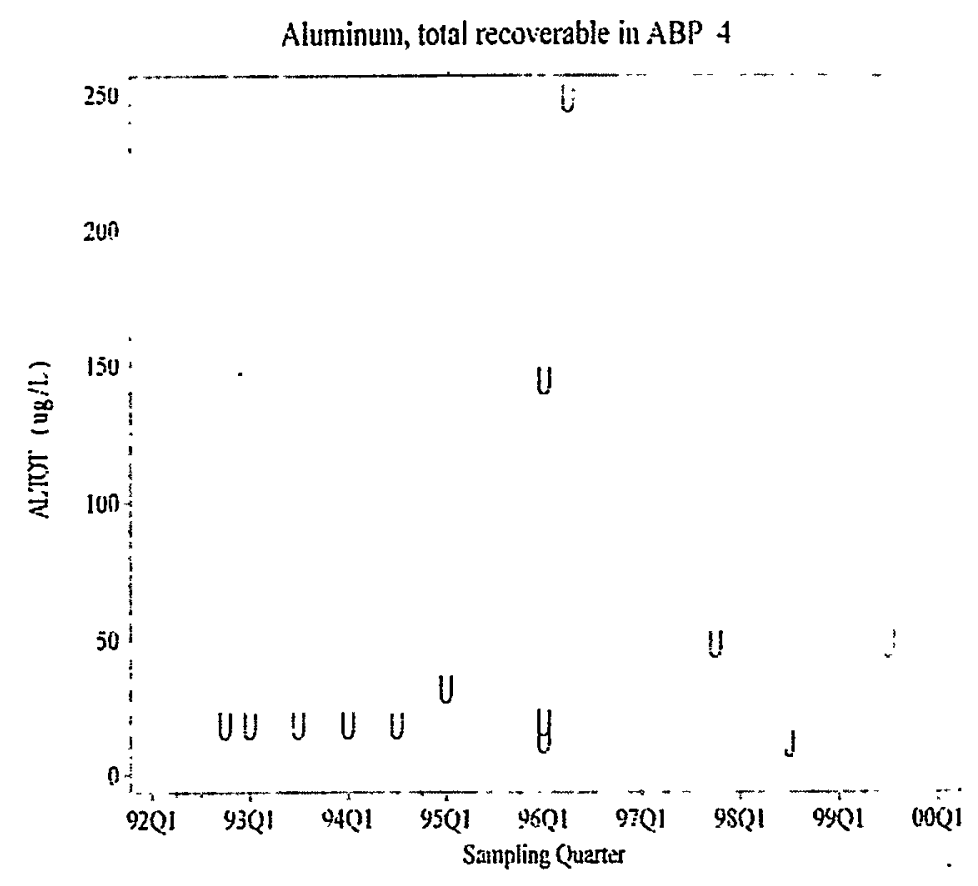

(Plot symbol: Res(ual if exists or ît) 
Lead, total recoverable in ABP 4

Data Retrieved by AWSA v1 from GIMS on 09NOV99 at 15:50

\begin{tabular}{lllllr} 
Well & Analyte & \multicolumn{2}{c}{ Sample_Q Res_Qual Result } & Units \\
& & & & \\
ABP 4 & PBTOT & $1992 Q 4$ & $U$ & 3 UGL \\
ABP 4 & PBTOT & $1993 Q 1$ & $U$ & 3 UGL \\
ABP 4 & PBTOT & $1993 Q 1$ & $U$ & 3 UGL \\
ABP 4 & PBTOT & $1993 Q 1$ & $U$ & 3 UGL \\
ABP 4 & PBTOT & $1993 Q 1$ & $U$ & 3 UGL \\
ABP 4 & PBTOT & $1993 Q 1$ & $U$ & 3 UGL \\
ABP 4 & PBTOT & $1993 Q 1$ & $U$ & 3 UGL \\
ABP 4 & PBTOT & $1993 Q 3$ & $U$ & 3 UGL \\
ABP 4 & PBTOT & $1994 Q 1$ & $U$ & 3 UGL \\
ABP 4 & PBTOT & $1994 Q 1$ & $U$ & 3 UGL \\
ABP 4 & PBTOT & $1994 Q 3$ & $U$ & 3 UGL \\
ABP 4 & PBTOT & 199501 & $U$ & 5 UGL \\
ABP 4 & PBTOT & $1996 Q 1$ & $U$ & 3.5 UGL \\
ABP 4 & PBTOT & $1996 Q 1$ & $U$ & 15 UGL \\
ABP 4 & PBTOT & $1996 Q 1$ & $U$ & 47 UGL \\
ABP 4 & PBTOT & $1996 Q 1$ & $U$ & 47 UGL \\
ABP 4 & PBTOT & $1996 Q 1$ & $U$ & 47 UGL \\
ABP 4 & PBTOT & $1996 Q 2$ & & 1.62 UGL \\
ABP 4 & PBTOT & $1997 Q 4$ & & 14.7 UGL \\
ABP 4 & PBTOT & $1998 Q 3$ & $J$ & 0.464 UGL \\
ABP 4 & PBTOT & $1999 Q 3$ & $U$ & 5 UGL \\
ABP 4 & PBTOT & $1999 Q 3$ & $U$ & 5 UGL
\end{tabular}

Letad. total recoverable in ABP 4

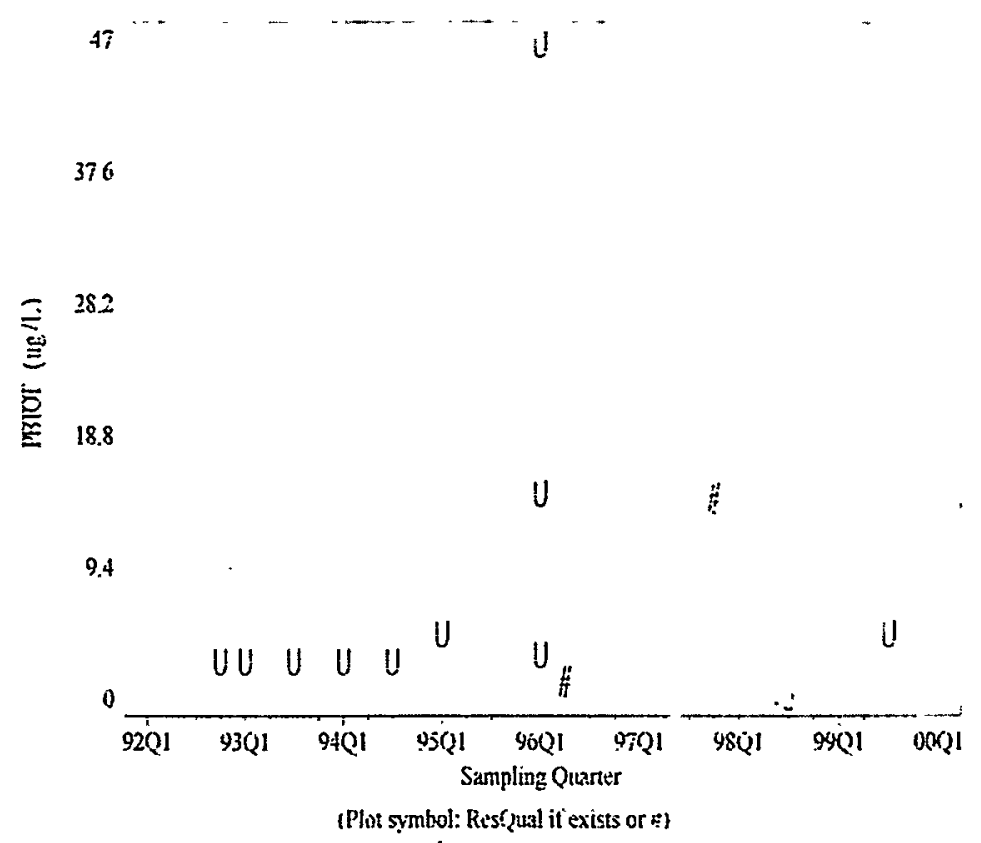


Chloride in ABP 4

Data Retrieved by AWSA v1 from GIMS on 09NOV99 at 15:50

\begin{tabular}{|c|c|c|c|c|}
\hline Well & Analyte & Sample_Q Res_Qual & Result & Units \\
\hline ABP 4 & CL & 1985Q1 & 1140 & 0 UGL \\
\hline ABP 4 & $C L$ & 198502 & 1490 & 0 UGL \\
\hline ABP 4 & $\mathrm{CL}$ & $1985 Q 3$ & 1600 & 0 UGL \\
\hline ABP 4 & CL & $1985 Q 4$ & 1760 & 0 UGL \\
\hline ABP 4 & $\mathrm{CL}$ & $1985 Q 4$ & 2940 & 0 UGL \\
\hline ABP 4 & $\mathrm{CL}$ & 198504 & 3530 & 0 UGL \\
\hline ABP 4 & $\mathrm{CL}$ & 1986Q1 & 6100 & 0 UGL \\
\hline ABP 4 & CL & 1987Q1 & 2500 & 0 UGL \\
\hline ABP 4 & $\mathrm{CL}$ & $1989 Q 2$ & 4000 & 0 UGL \\
\hline ABP 4 & CL & $1989 Q 3$ & 1400 & 0 UGL \\
\hline ABP 4 & $C L$ & $1980 Q 3$ & 1600 & 0 UGL \\
\hline ABP 4 & CL & 199004 & 1550 & 0 UGL \\
\hline ABP 4 & CL & $1990 Q 4$ & 1550 & 0 UGL \\
\hline ABP 4 & CL & $1990 Q 4$ & 1800 & 0 UGL \\
\hline ABP 4 & CL & 199004 & 1800 & O UGL \\
\hline ABP 4 & CL & 1991Q1 & 1670 & 0 UGL \\
\hline ABP 4 & CL & 1991Q2 & 2900 & 0 UGL \\
\hline ABP 4 & $\mathrm{CL}$ & 1991Q3 & 1860 & 0 UGL \\
\hline ABP 4 & $\mathrm{CL}$ & 1991Q4 & 1630 & 0 UGL \\
\hline ABP 4 & $\mathrm{CL}$ & 1992Q1 & 1720 & O UGL \\
\hline ABP 4 & CL & 1993Q1 & 1290 & 0 UGL \\
\hline ABP 4 & $C L$ & 1993Q1 & 1370 & 0 UGL \\
\hline ABP 4 & CL & 1993Q1 & 1450 & 0 UGL \\
\hline ABP 4 & CL & 1993Q1 & 1460 & \\
\hline ABP 4 & CL & 199301 & 1460 & O UGL \\
\hline ABP 4 & $C L$ & $1997 Q 4$ & 1580 & 0 UGL \\
\hline ABP 4 & CL & $1998 Q 3$ & 1470 & 0 UGL \\
\hline ABP 4 & CL & $1998 Q 3$ & 1480 & 0 UGL \\
\hline ABP 4 & $\mathrm{CL}$ & $1999 Q 3$ & 1310 & 0 UGL \\
\hline ABP 4 & CL & 1999Q3 & 1380 & \\
\hline
\end{tabular}

Chloride in ABP 4

(Plot symbal Res(ual if wists or 
Carbon tetrachloride in ABP 4

Data Retrieved by AWSA v1 from GIMS on 09NOV99 at 15:50

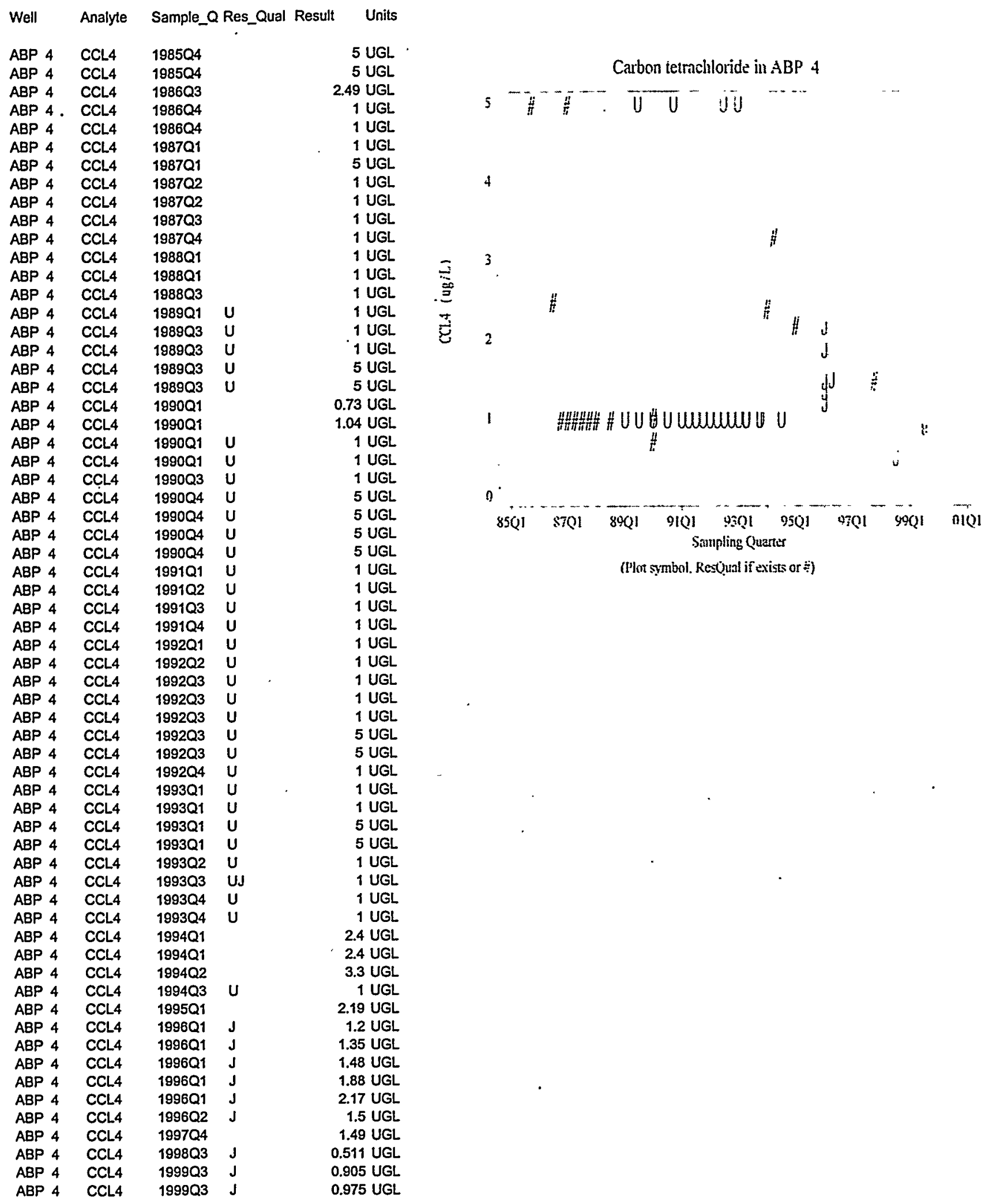


Tetrachloroethylene in ABP 4

Data Retrieved by AWSA v1 from GIMS on 09NOV99 at 15:50

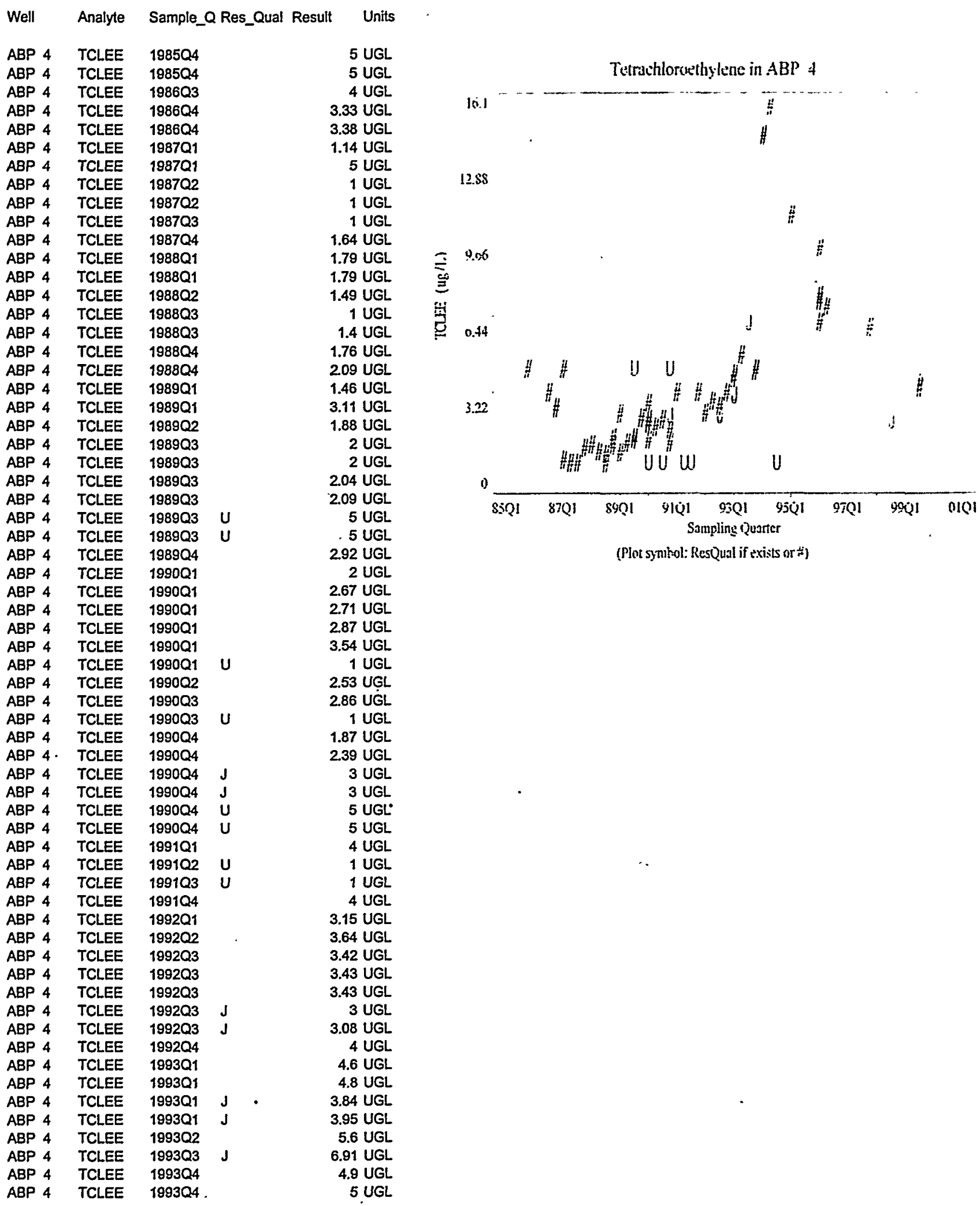


Tetrachloroethylene in ABP 4

Data Retrieved by AWSA v1 from GIMS on 09NOV99 at 15:50

\begin{tabular}{|c|c|c|c|c|c|}
\hline Well & Analyte & Sample_ & Res_Qual & Result & Unils \\
\hline ABP 4 & TCLEE & 1994Q1 & & & $1.8 \mathrm{UGL}$ \\
\hline $\mathrm{ABP} 4$ & TCLEE & 1994Q1 & & 14.8 & 1.9 UGL \\
\hline ABP 4 & TCLEE & $1994 Q 2$ & & 16. & 3.1 UGL \\
\hline$A B P 4$ & TCLEE & $1994 Q 3$ & $U$ & & 1 UGL \\
\hline$A B P 4$ & TCLEE & 1995Q1 & & 11.5 & 1.5 UGL \\
\hline ABP 4 & TCLEE & 1996Q1 & & 6.98 & 96 UGL \\
\hline ABP 4 & TCLEE & 1996Q1 & & 7.85 & 85 UGL \\
\hline$A B P 4$ & TCLEE & 199601 & & 7.87 & 87 UGL \\
\hline ABP 4 & TCLEE & 1996Q1 & & & $3.1 \mathrm{UGL}$ \\
\hline ABP 4 & TCLEE & 1996Q1 & & 10. & 0.1 UGL \\
\hline ABP 4 & TCLEE & 199602 & & 7.6 & 61 UGL \\
\hline$A B P_{4}$ & TCLEE & $1997 Q 4$ & & 6.7 & 74 UGL \\
\hline ABP 4 & TCLEE & $1998 Q 3$ & $J$ & $2.7 t$ & 76 UGL \\
\hline ABP 4 & TCLEE & 1999Q3 & & & 1.2 UGL \\
\hline ABP 4 & TCLEE & 1999Q3 & & & 1.4 UGL \\
\hline
\end{tabular}


Trichloroethylene in ABP 4

Data Relrieved by AWSA v1 from GIMS on 09NOV99 at 15:50

\begin{tabular}{|c|c|c|c|c|c|c|}
\hline Well & & Analyte & Sample_C & Res_Qual & Result & Units \\
\hline$A B P$ & 4 & TRCLE & $1985 Q 4$ & & & 5 UGL \\
\hline$A B P$ & 4 & TRCLE & $1985 Q 4$ & & & 5 UGL \\
\hline$A B P$ & 4 & TRCLE & 1986Q2 & & & 3.55 UGL \\
\hline$A B P$ & 4 & TRCLE & 1986 Q3 & & & 3.67 UGL \\
\hline$A B P$ & 4 & TRCLE & $1986 Q_{4}$ & & . & 5.26 UGL \\
\hline$A B P$ & 4 & TRCLE & $1986 Q 4$ & & & 5.78 UGL \\
\hline ABP & 4 & TRCLE & 1987Q1 & & & 3.44 UGL \\
\hline$A B P$ & 4 & TRCLE & $1987 Q 1$ & & & 5 UGL \\
\hline $\mathrm{ABP}$ & 4 & TRCLE & $1987 Q 2$ & & & 6.2 UGL \\
\hline$A B P$ & 4 & TRCLE & 1987Q2 & & & 6.9 UGL \\
\hline$A B P$ & 4 & TRCLE & 1987Q3 & & & $1 \mathrm{UGL}$ \\
\hline$A B P$ & 4 & TRCLE & 198704 & & & 6 UGL \\
\hline$A B P$ & 4 & TRCLE & 1988Q1 & & & 4.18 UGL \\
\hline$A B P$ & 4 & TRCLE & 1988Q1 & & & 4.18 UGL \\
\hline ABP & 4 & TRCLE & $1988 Q 2$ & & & 3.9 UGL \\
\hline $\mathrm{ABP}$ & 4 & TRCLE & 1888Q3 & & & 2.86 UGL \\
\hline$A B P$ & 4 & TRCLE & 1988Q3 & & & 3.66 UGL \\
\hline$A B P$ & 4 & TRCLE & $1888 Q 4$ & & & 4.15 UGL \\
\hline ABP & 4 & TRCLE & $1988 Q 4$ & & & 4.92 UGL \\
\hline$A B P$ & 4 & TRCLE & $1989 Q 1$ & & & 5.05 UGL \\
\hline$A B P$ & 4 & TRCLE & $1989 Q 1$ & & & 8.59 UGL \\
\hline$A B P$ & 4 & TRCLE & 1989Q2 & & & 4.87 UGL \\
\hline$A B P$ & 4 & TRCLE & 1989Q3 & & & 5 UGL \\
\hline$A B P$ & 4 & TRCLE & $1989 Q 3$ & & & 5 UGL \\
\hline$A B P$ & 4 & TRCLE & 1989Q3 & & & 5.08 UGL \\
\hline$A B P$ & 4 & TRCLE & $1989 Q 3$ & & & 5.24 UGL \\
\hline$A B P$ & 4 & TRCLE & $1989 Q 3$ & & & 6 UGL \\
\hline $\mathrm{ABP}$ & 4 & TRCLE & 1989Q3 & J & & 5 UGL \\
\hline ABP & 4 & TRCLE & 1989Q4 & & & 8.48 UGL \\
\hline ABP & 4 & TRCLE & 1990Q1 & & & 5.55 UGL \\
\hline$A B P$ & 4 & TRCLE & 1990Q1 & & & 5.75 UGL \\
\hline ABP & 4 & TRCLE & 1990Q1 & & & 6 UGL \\
\hline$A B P$ & 4 & TRCLE & 1990Q1 & & & $6.56 \mathrm{UGL}$ \\
\hline$A B P$ & 4 & TRCLE & 1990Q1 & & & 7.97 UGL \\
\hline$A B P$ & 4 & TRCLE & 1990Q1 & $\mathbf{U}$ & & 1 UGL \\
\hline $\mathrm{ABP}$ & 4 & TRCLE & 199002 & & & 6.31 UGL \\
\hline ABP & 4 & TRCLE & $1990 Q 3$ & & & 7 UGL \\
\hline$A B P$ & 4 & TRCLE & $1990 Q 3$ & & & 7.43 UGL \\
\hline $\mathrm{ABP}$ & 4 & TRCLE & 199004 & & & 5.3 UGL \\
\hline$A B P$ & 4 & TRCLE & $1990 Q 4$ & & & $6 \mathrm{UGL}$ \\
\hline ABP & 4 & TRCLE & 1990Q4 & & & 6 UGL \\
\hline $\mathrm{ABP}$ & 4 & TRCLE & $1990 Q 4$ & & & 6.88 UGL \\
\hline$A B P$ & 4 & TRCLE & 189004 & & & 8 UGL \\
\hline$A B P$ & 4 & TRCLE & $1990 Q_{4}$ & & & 8 UGL \\
\hline$A B P$ & 4 & TRCLE & 1991Q1 & & & 8 UGL \\
\hline$A B P$ & 4 & TRCLE & 1991Q2 & $\mathbf{U}$ & & 1 UGL \\
\hline$A B P$ & 4 & TRCLE & 1991Q3 & & & 11 UGL \\
\hline$A B P$ & 4 & TRCLE & 1991Q4 & & & 11 UGL \\
\hline$A B P$ & 4 & TRCLE & 1992Q1 & & & 9.32 UGL \\
\hline ABP & 4 & TRCLE & 1992Q2 & & & 9.39 UGL \\
\hline$A B P$ & 4 & TRCLE & 1992Q3 & & & $9.7 \mathrm{UGL}$ \\
\hline$A B P$ & 4 & TRCLE & $1992 Q 3$ & & & 9.76 UGL \\
\hline$A B P$ & 4 & TRCLE & 1992Q3 & & & 10.4 UGL \\
\hline$A B P$ & 4 & TRCLE & 1992Q3 & & & 10.5 UGL \\
\hline$A B P$ & 4 & TRCLE & 1992Q3 & & & 10.8 UGL \\
\hline$A B P$ & 4 & TRCLE & $1992 Q 4$ & & & 11.9 UGL \\
\hline ABP & 4 & TRCLE & 1993Q1 & & & 14.5 UGL \\
\hline$A B P$ & 4 & TRCLE & 1993Q1 & & & 15 UGL \\
\hline ABP & 4 & TRCLE & 1993Q1 & & & 15 UGL \\
\hline ABP & 4 & TRCLE & 1993Q1 & & & 16.7 UGL \\
\hline$A B P$ & 4 & TRCLE & $1993 \mathrm{Q} 2$ & & & 23 UGL \\
\hline ABP & 4 & TRCLE & $1993 Q 3$ & $J$ & & 30.2 UGL \\
\hline$A B P$ & 4 & TRCLE & $1993 Q 4$ & & & $12.3 \mathrm{UGL}$ \\
\hline
\end{tabular}

Trichloroethylene in $\mathrm{ABP} A$

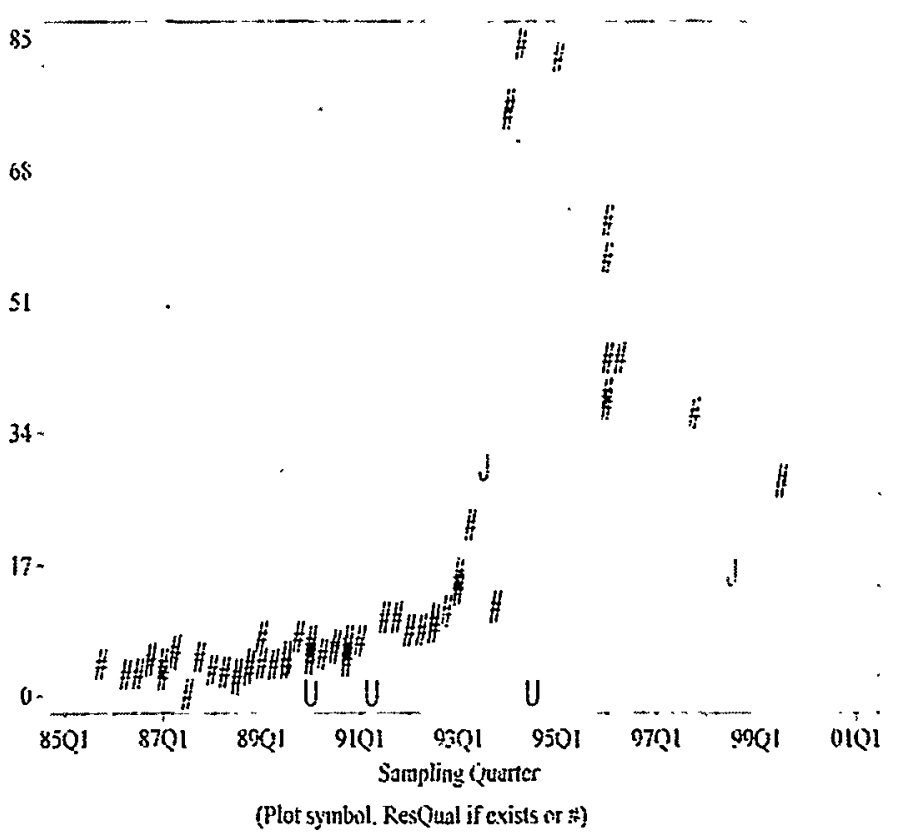


Trichloroethylene in ABP 4

Data Retrieved by AWSA v1 from GIMS on 09NOV99 at 15:50

\begin{tabular}{|c|c|c|c|c|}
\hline Well & Analyte & Sample_C & Res_Qual & Result \\
\hline ABP 4 & TRCLE & $1993 Q 4$ & & 12.5 UGL \\
\hline ABP 4 & TRCLE & 1994Q1 & & 76 UGL \\
\hline$A B P 4$ & TRCLE & 1994Q1 & & 77.3 UGL \\
\hline ABP 4 & TRCLE & 199402 & & 85 UGL \\
\hline$A B P 4$ & TRCLE & $1994 Q 3$ & $u$ & 1 UGL \\
\hline$A B P 4$ & TRCLE & $1995 \mathrm{Q} 1$ & & 83.4 UGL \\
\hline ABP 4 & TRCLE & $1996 Q 1$ & & 38.4 UGL \\
\hline$A B P 4$ & TRCLE & 1996Q1 & & 39.9 UGL \\
\hline ABP 4 & TRCLE & 199601 & & 44.4 UGL \\
\hline$A B P 4$ & TRCLE & $1996 \mathrm{Q} 1$ & & 57.4 UGL \\
\hline$A B P 4$ & TRCLE & 199601 & & 62.3 UGL \\
\hline $\mathrm{ABP}_{4}$ & TRCLE & 199602 & & $44.4 \mathrm{UGL}$ \\
\hline ABP 4 & TRCLE & $1997 Q 4$ & & 37.2 UGL \\
\hline ABP 4 & TRCLE & 199803 & $J$ & 16.7 UGL \\
\hline ABP 4 & TRCLE & 1999Q3 & & 28.4 UGL \\
\hline ABP 4 & TRCLE & 199903 & & 28.8 UGL \\
\hline
\end{tabular}


Gross alpha in ABP 4

Data Retrieved by AWSA v1 from GIMS on 09NOV99 at 15:50

\begin{tabular}{|c|c|c|c|c|c|c|}
\hline Well & & Analyte & Sample_ & Res_Qual & Result & Units \\
\hline ABP & 4 & ALPHAG & 1985Q1 & & & $0.8 \mathrm{PCL}$ \\
\hline$A B P$ & 4 & ALPHAG & 1985Q2 & & & $2 \mathrm{PCl}$ \\
\hline ABP & 4 & ALPHAG & 1985Q3 & & & $10 \mathrm{PCL}$ \\
\hline ABP & 4 & ALPHAG & 1985Q4 & & & $2 \mathrm{PCl}$ \\
\hline ABP & 4 & ALPHAG & $1985 Q 4$ & & & $5 \mathrm{PCL}$ \\
\hline$A B P$ & 4 & ALPHAG & 1986Q1 & & & $2 \mathrm{PCl}$ \\
\hline$A B P$ & 4 & ALPHAG & 1987Q1 & & & $1.9 \mathrm{PCL}$ \\
\hline$A B P$ & 4 & ALPHAG & 1987Q1 & & & $3 \mathrm{PCL}$ \\
\hline ABP & 4 & ALPHAG & 1988Q1 & & & 5.4 PCL \\
\hline$A B P$ & 4 & ALPHAG & 1988Q1 & & & $5.4 \mathrm{PCL}$ \\
\hline$A B P$ & 4 & ALPHAG & 1989Q1 & & & $2.33 \mathrm{PCL}$ \\
\hline$A B P$ & 4 & ALPHAG & 1989Q1 & & & $2.36 \mathrm{PCL}$ \\
\hline ABP & 4 & ALPHAAG & 1989Q2 & $\mathbf{U}$ & & $3 \mathrm{PCL}$ \\
\hline ABP & 4 & ALPHAG & 1989Q3 & $\mathbf{U}$ & & $3 \mathrm{PCL}$ \\
\hline$A B P$ & 4 & ALPHAG & 1990Q1 & $\mathbf{U}$ & & $3 \mathrm{PCL}$ \\
\hline$A B P$ & 4 & ALPHAG & 1990Q1 & $\mathbf{U}$ & & 3 PCL \\
\hline ABP & 4 & ALPHAG & 1990Q1 & $\mathbf{U}$ & & $3 \mathrm{PCL}$ \\
\hline ABP & 4 & ALPHAG & 199001 & $U$ & & $3 \mathrm{PCL}$ \\
\hline ABP & 4 & ALPHAG & 1990Q3 & U & & 2 PCL \\
\hline ABP & 4 & ALPHAG & 199004 & U & & $2 \mathrm{PCL}$ \\
\hline ABP & 4 & ALPHAG & $1990 Q 4$ & U & & $2 \mathrm{PCL}$ \\
\hline ABP & 4 & ALPHAG & 199004 & $\mathbf{U}$ & & $3 \mathrm{PCL}$ \\
\hline ABP & 4 & ALPHAG & 1990Q4 & $\mathbf{U}$ & & $4 \mathrm{PCL}$ \\
\hline ABP & 4 & ALPHAG & 1991Q1 & $U$ & & $2 \mathrm{PCL}$ \\
\hline$A B P$ & 4 & ALPHAG & 1991Q2 & $\mathbf{U}$ & & $2 \mathrm{PCL}$ \\
\hline$A B P$ & 4 & ALPHAG & 1991Q3 & $\mathbf{U}$ & & $2 \mathrm{PCL}$ \\
\hline ABP & 4 & ALPHAG & 199104 & $\mathbf{U}$ & & $2 \mathrm{PCL}$ \\
\hline ABP & 4 & ALPHAG & 1992Q1 & $\mathbf{U}$ & & 2 PCL \\
\hline$A B P$ & 4 & ALPHAG & $1992 Q 3$ & & & $2.3 \mathrm{PCL}$ \\
\hline ABP & 4 & ALPHAG & 1992Q3 & & & $2.9 \mathrm{PCL}$ \\
\hline$A B P$ & 4 & ALPHAG & 1992Q3 & & & 3 PCL \\
\hline$A B P$ & 4 & ALPHAG & 1993Q1 & & & 1.9 PCL \\
\hline ABP & 4 & ALPHAG & 1993Q1 & . & & 1.9 PCL \\
\hline$A B P$ & 4 & ALPHAG & 1893Q1 & & & $2.6 \mathrm{PCL}$ \\
\hline$A B P$ & 4 & ALPHAG & 1993Q1 & & & $2.86 \mathrm{PCL}$ \\
\hline$A B P$ & 4 & ALPHAG & 1893Q1 & $\mathbf{U}$ & & $2 \mathrm{PCL}$ \\
\hline$A B P$ & 4 & ALPHAG & 1994Q3 & & & $1.94 \mathrm{PCL}$ \\
\hline ABP & 4 & ALPHAG & 1995Q1 & & & $1.29 \mathrm{PCL}$ \\
\hline ABP & 4 & ALPHAG & 1996Q1 & & & $0.837 \mathrm{PCL}$ \\
\hline ABP & 4 & ALPHAG & 1996Q1 & & * & $1.04 \mathrm{PCL}$ \\
\hline ABP & 4 & ALPHAG & 1996Q1 & & & $2.02 \mathrm{PCL}$ \\
\hline ABP & 4 & ALPHAG & 1996Q1 & & & $2.03 \mathrm{PCL}$ \\
\hline ABP & 4 & ALPHAG & 1996Q1 & Ul & & 0.82 PCL \\
\hline ABP & 4 & ALPHAG & 1996Q2 & & & $1.08 \mathrm{PCL}$ \\
\hline$A B P$ & 4 & ALPHAG & 1997Q4 & & & $2.4 \mathrm{PCL}$ \\
\hline$A B P$ & 4 & ALPHAG & 1998Q3 & & & $0.794 \mathrm{PCL}$ \\
\hline ABP & 4 & ALPHAG & 1999Q3 & $\mathbf{J}$ & & $1.08 \mathrm{PCL}$ \\
\hline ABP & 4 & ALPHAG & 1999Q3 & $J$ & & $1.46 \mathrm{PCL}$ \\
\hline
\end{tabular}

Gross alpha in $A B P 4$

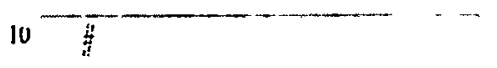

$\mathbf{S}$

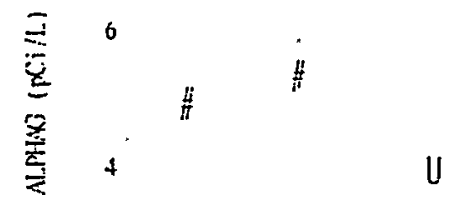

2

(Plot symbol: ResQual if exists or 
1,1,1-Trichloroethane in ABP 4

Data Retrieved by AWSA v1 from GIMS on 09NOV99 at 15:50

\begin{tabular}{|c|c|c|c|c|}
\hline Well & Analyte & Sample_C & Res_Qual Result & Units \\
\hline ABP 4 & 111TCE & $1985 Q 4$ & & 5 UGL \\
\hline ABP 4 & 111TCE & 198504 & & 5 UGL \\
\hline ABP 4 & 111TCE & 198603 & & 1 UGL \\
\hline ABP 4 & 111TCE & $1986 Q 4$ & & 1 UGL \\
\hline ABP 4 & 111TCE & $1986 Q 4$ & & 2.6 UGL \\
\hline ABP 4 & 111TCE & 1987Q1 & & 1 UG \\
\hline ABP 4 & 111TCE & 1987Q1 & & 5 UGL \\
\hline ABP 4 & 111TCE & 198702 & & 1 UGL \\
\hline ABP 4 & 111TCE & 1987Q2 & & 1 UG \\
\hline ABP 4 & 111TCE & 198703 & & 1 UGL \\
\hline ABP 4 & 111TCE & 198704 & & \\
\hline ABP 4 & 111TCE & 198801 & & $1 . \mathrm{UG}$ \\
\hline ABP 4 & 111TCE & 198801 & & 1 UGL \\
\hline ABP 4 & 111TCE & 198802 & & \\
\hline ABP 4 & 111TCE & 1988Q3 & & 1 UGL \\
\hline ABP 4 & 111TCE & $1988 Q 3$ & & 1 UGL \\
\hline ABP 4 & 111TCE & $1988 Q 4$ & U & 1 UG \\
\hline ABP 4 & 111TCE & 1988Q4 & U & 1 UG \\
\hline ABP 4 & 111TCE & 188901 & $\mathbf{U}$ & 1 UGL \\
\hline ABP 4 & 111TCE & 1989Q1 & U & 1 UG \\
\hline ABP 4 & 111TCE & $1989 Q 2$ & U & \\
\hline ABP 4 & 111TCE & $1989 Q 3$ & U & 1 UGL \\
\hline ABP 4 & 111TCE & 1989Q3 & $\mathbf{U}$ & 1 UG \\
\hline ABP 4 & 111TCE & $1989 Q 3$ & $\mathbf{u}$ & \\
\hline ABP 4 & 111TCE & $1989 Q 3$ & $\mathbf{u}$ & 1 UGL \\
\hline ABP 4 & 111TCE & 1989Q3 & $u$ & 5 UG \\
\hline ABP 4 & 111TCE & 198903 & $u$ & 5 UG \\
\hline ABP 4 & 111TCE & 1989Q4 & $\mathbf{u}$ & 1 UGL \\
\hline ABP 4 & 111TCE & 1990Q1 & $u$ & 0.4 UGL \\
\hline ABP 4 & 111TCE & $1990 \mathrm{Q1}$ & u & $0.4 \mathrm{C}$ \\
\hline ABP 4 & 111TCE & $1990 Q 1$ & U & 1 UGL \\
\hline $\mathrm{ABP}_{4}$ & 111TCE & $1990 Q 1$ & $U$ & 1 UG \\
\hline ABP 4 & 111TCE & 199001 & $u$ & \\
\hline ABP 4 & 111TCE & $1990 Q 1$ & $u$ & 11 \\
\hline ABP 4 & 111TCE & 189002 & $U$ & 1 UGL \\
\hline ABP 4 & 111TCE & 199003 & $U$ & \\
\hline ABP 4 & 111TCE & 1990Q3 & $U$ & 14 \\
\hline ABP 4 & 111TCE & $1990 Q 4$ & $u$ & \\
\hline ABP 4 & 111TCE & $1990 Q 4$ & U & \\
\hline $\mathrm{ABP}_{4}$ & 111TCE & 199004 & $u$ & \\
\hline ABP 4 & 111TCE & 1990Q4 & $U$ & \\
\hline ABP 4 & 111TCE & 199004 & $U$ & \\
\hline ABP 4 & 111TCE & 199004 & $U$ & \\
\hline ABP 4 & 111TCE & 1991Q1 & $U$ & \\
\hline ABP 4 & 111TCE & 199102 & U & \\
\hline ABP 4 & 111TCE & 1991Q3 & U & 1 \\
\hline $\mathrm{ABP} 4$ & 111TCE & 1991Q4 & $\mathbf{u}$ & \\
\hline ABP 4 & 111TCE & 1992Q1 & U & \\
\hline ABP 4 & 111TCE & $1992 Q 2$ & U & \\
\hline ABP 4 & 111TCE & $1992 Q 3$ & U & \\
\hline ABP 4 & 111TCE & 1992Q3 & U & \\
\hline ABP 4 & 111TCE & $1992 Q 3$ & U & \\
\hline ABP 4 & 111TCE & 1992Q3 & $u$ & \\
\hline$A B P 4$ & 111TCE & $1992 Q 3$ & $U$ & \\
\hline ABP 4 & 111TCE & 1992Q4 & $u$ & \\
\hline ABP 4 & 111TCE & 1993Q1 & u & \\
\hline ABP 4 & 1111TCE & $1993 Q 1$ & $u$ & \\
\hline ABP 4 & 111TCE & 1893Q1 & U & \\
\hline ABP 4 & 111TCE & 1993Q1 & $u$ & \\
\hline ABP 4 & 111TCE & 199302 & $u$ & \\
\hline ABP 4 & 111TCE & 199303 & UJ & \\
\hline ABP 4 & 111TCE & 1993Q4 & $u$ & \\
\hline ABP 4 & 111TCE & 199304 & u & \\
\hline ABP 4 & 111TCE & 1994Q1 & $\mathbf{u}$ & \\
\hline ABP 4 & 111TCE & $1994 Q 1$ & $u$ & \\
\hline ABP 4 & 111TCE & $1994 Q 2$ & $U$ & \\
\hline ABP 4 & 111TCE & 199403 & $u$ & \\
\hline ABP 4 & 111TCE & 199501 & $u$ & \\
\hline
\end{tabular}

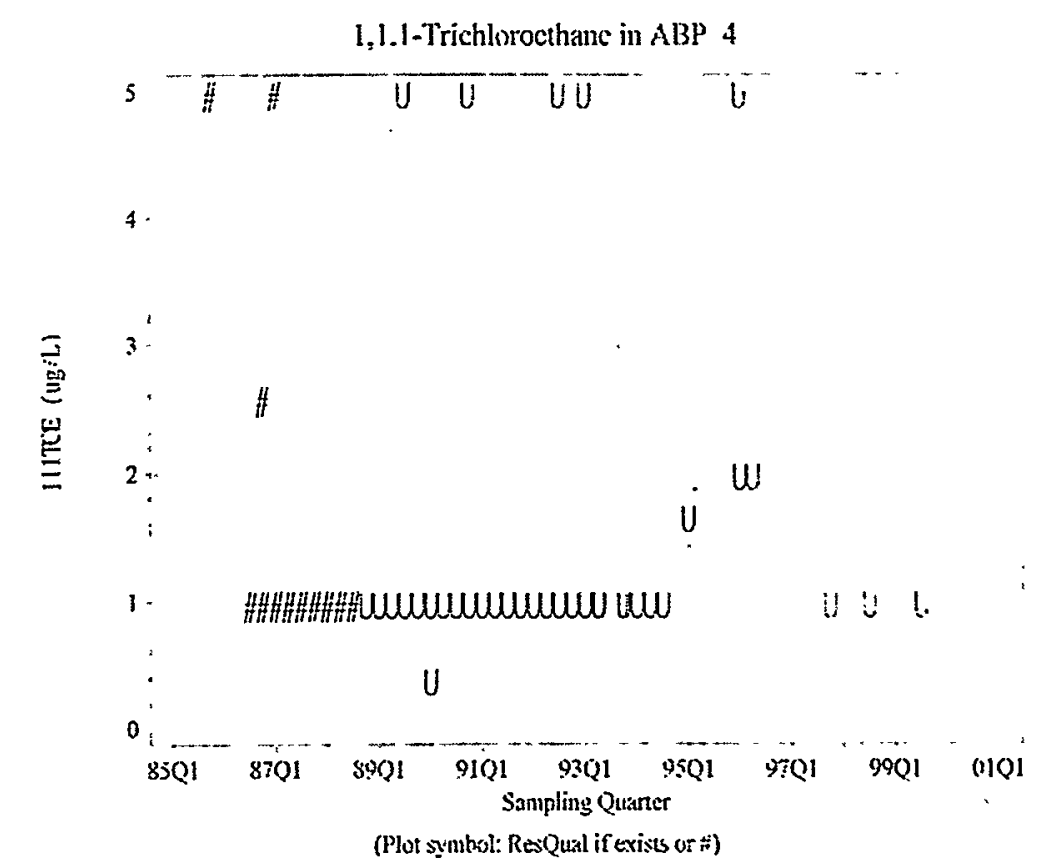

(Plot symbal: ResQual if exists on 
1,1,1-Trichloroethane in ABP 4

Data Retrieved by AWSA v1 from GIMS on 09NOV99 at 15:50

\begin{tabular}{lllll} 
Well & Analyte & \multicolumn{2}{c}{ Sample_Q Res_Qual Result } & Units \\
& & & \\
ABP 4 & 111 TCE & $1996 Q 1$ & $U$ & 2 UGL \\
ABP 4 & $111 T C E$ & $1996 Q 1$ & $U$ & 2 UGL \\
ABP 4 & $111 T C E$ & $1996 Q 1$ & $U$ & 2 UGL \\
ABP 4 & $111 T C E$ & $1996 Q 1$ & $U$ & 5 UGL \\
ABP 4 & $111 T C E$ & $1996 Q 1$ & $U$ & 5 UGL \\
ABP 4 & $111 T C E$ & 199622 & $U$ & 2 UGL \\
ABP 4 & $111 T C E$ & $1997 Q 4$ & $U$ & 1 UGL \\
ABP 4 & $111 T C E$ & $1998 Q 3$ & $U$ & 1 UGL \\
ABP 4 & $111 T C E$ & $1999 Q 3$ & $U$ & 1 UGL \\
ABP 4 & $111 T C E$ & $1999 Q 3$ & $U$ & 1 UGL
\end{tabular}


1,1-Dichloroethylene in ABP 4

Data Retrieved by AWSA v1 from GIMS on 09NOV99 at 15:50

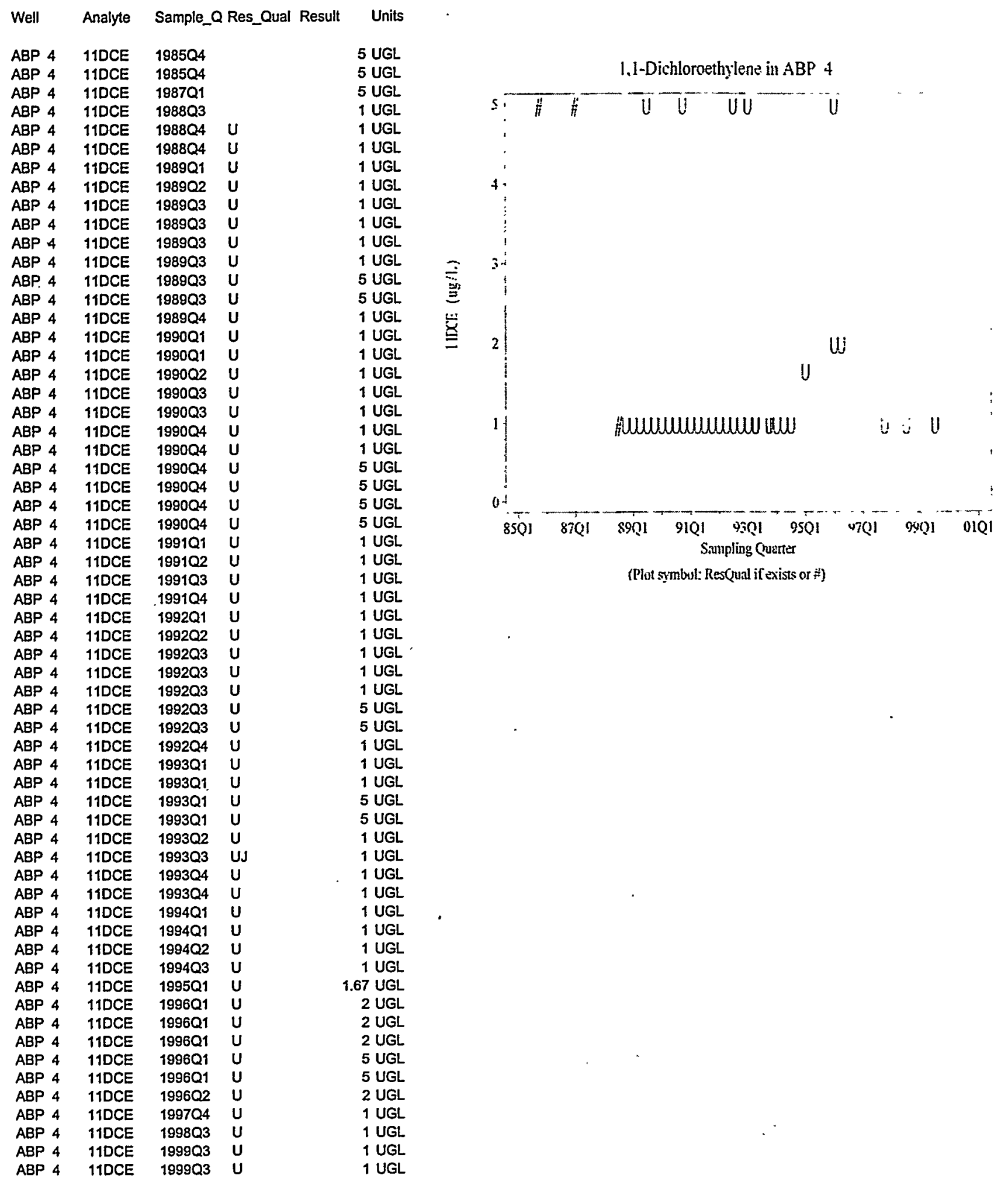


1,1-Dichloroethane in ABP 4

Data Retrieved by AWSA v1 from GIMS on 09NOV99 at 15:50

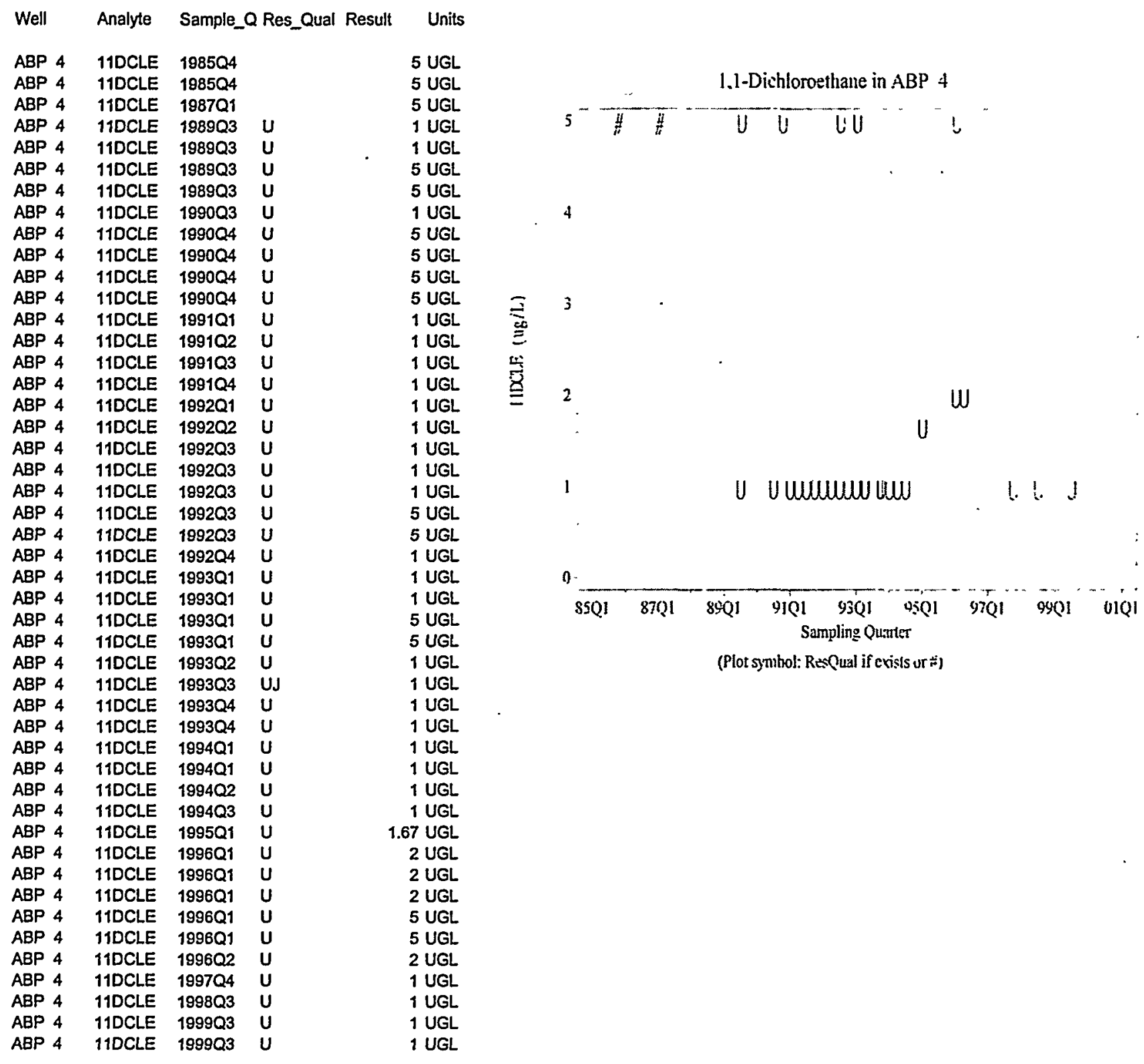


Barium, total recoverable in ABP 4

Data Retrieved by AWSA v1 from GIMS on 09NOV99 at 15:50

\begin{tabular}{llll} 
Well & Analyte & \multicolumn{2}{c}{ Sample_Q Re } \\
& & & \\
ABP 4 & BATOT & $1993 Q 1$ & \\
ABP 4 & BATOT & $1993 Q 1$ & \\
ABP 4 & BATOT & $1993 Q 1$ & $U$ \\
ABP 4 & BATOT & $1993 Q 1$ & $U$ \\
ABP 4 & BATOT & $1993 Q 1$ & $U$ \\
ABP 4 & BATOT & $1994 Q 3$ & $U$ \\
ABP 4 & BATOT & $1995 Q 1$ & $U$ \\
ABP 4 & BATOT & $1996 Q 1$ & \\
ABP 4 & BATOT & $1996 Q 1$ & \\
ABP 4 & BATOT & $1996 Q 1$ & \\
ABP 4 & BATOT & $1996 Q 1$ & $\mathrm{~J}$ \\
ABP 4 & BATOT & $1996 Q 1$ & $\mathrm{~J}$ \\
ABP 4 & BATOT & $1996 Q 2$ & $\mathrm{U}$ \\
ABP 4 & BATOT & $1997 Q 4$ & $\mathrm{~J}$ \\
ABP 4 & BATOT & 199803 & \\
ABP 4 & BATOT & $1999 Q 3$ & $\mathrm{~J}$ \\
ABP 4 & BATOT & $1999 Q 3$ & $\mathrm{~J}$
\end{tabular}

3.35 UGL 3.41 UGL 4 UGL 4 UGL 4 UGL 3 UGL 5 UGL 2.4 UGL 2.4 UGL 2.9 UGL 2.49 UGL 2.56 UGL 25 UGL 2.53 UGL 2.56 UGL 2.3 UGL 2.37 UGL
Barium. total recoverable in $\mathrm{ABP} 4$

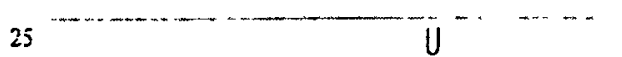

20

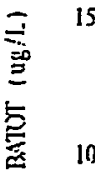

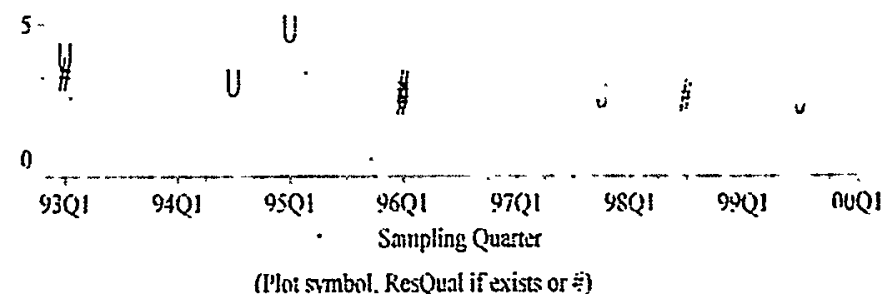


Nonvolatile beta in ABP 4

Data Retrieved by AWSA v1 from GIMS on 09NOV99 at 15:50

\begin{tabular}{|c|c|c|c|c|}
\hline Well & Analyte & Sample_c & Res_Qual & Result \\
\hline ABP 4 & BETAG & 1985Q1 & & $1 \mathrm{PCl}$ \\
\hline $\mathrm{ABP} 4$ & BETAG & 1985Q2 & & $3 \mathrm{PCL}$ \\
\hline ABP 4 & BETAG & 1985Q3 & & $9 \mathrm{PCL}$ \\
\hline ABP 4 & BETAG & 1985Q4 & & 3 PC \\
\hline ABP 4 & BETAG & $1985 Q 4$ & & $7 \mathrm{PC}$ \\
\hline ABP 4 & BETAG & 1986Q1 & & $3 P C$ \\
\hline ABP 4 & BETAG & 1987Q1 & & $2 \mathrm{PCL}$ \\
\hline ABP 4 & BETAG & 1987Q1 & & $3 \mathrm{PCL}$ \\
\hline $\mathrm{ABP} 4$ & BETAG & 1988Q1 & & 3.9 PCL \\
\hline ABP 4 & BETAG & 1988Q1 & & $3.9 \mathrm{PCl}$ \\
\hline ABP 4 & BETAG & 198902 & $u$ & $2 P C$ \\
\hline ABP 4 & BETAG & $1989 Q 3$ & $u$ & $4 \mathrm{PC}$ \\
\hline ABP 4 & BETAG & 189003 & $u$ & $2 \mathrm{PC}$ \\
\hline ABP 4 & BETAG & 199004 & & 8.1 PCL \\
\hline$A B P 4$ & BETAG & $1890 Q 4$ & $\mathrm{U}$ & $2 \mathrm{PCL}$ \\
\hline$A B P 4$ & BETAG & 199004 & U & $2 \mathrm{PCL}$ \\
\hline ABP 4 & BETAG & $1990 Q 4$ & U & $5 \mathrm{PCL}$ \\
\hline ABP 4 & BETAG & 1991Q1 & U & $2 P$ \\
\hline ABP 4 & BETAG & 1991Q2 & $u$ & \\
\hline ABP 4 & BETAG & 1991Q3 & $u$ & 2 PC \\
\hline ABP 4 & BETAG & 1991Q4 & U & $2 \mathrm{PCl}$ \\
\hline ABP 4 & BETAG & 1992Q1 & U & $2 \mathrm{PC}$ \\
\hline ABP 4 & BETAG & 1992Q3 & & $1.4 \mathrm{PC}$ \\
\hline ABP 4 & BETAG & 1992Q3 & & $2.6 \mathrm{PCL}$ \\
\hline ABP 4 & BETAG & $1992 Q 3$ & & $2.7 \mathrm{PCl}$ \\
\hline ABP 4 & BETAG & 199203 & & $3.5 \mathrm{PCl}$ \\
\hline ABP 4 & BETAG & 199203 & $u$ & $2 \mathrm{PC}$ \\
\hline ABP 4 & BETAG & 1993Q1 & & $1.9 \mathrm{PCl}$ \\
\hline ABP 4 & BETAG & $1993 Q 1$ & & $2 \mathrm{PCl}$ \\
\hline ABP 4 & BETAG & 199301 & & $3.8 \mathrm{PCl}$ \\
\hline ABP 4 & BETAG & 1993Q1 & & 6.27 PCl \\
\hline ABP 4 & BETAG & 1993Q1 & U & $2 \mathrm{PCl}$ \\
\hline$A B P 4$ & BETAG & $1993 Q 3$ & $U$ & $2 \mathrm{PCl}$ \\
\hline ABP 4 & BETAG & 1994Q1 & & 2.3 PCl \\
\hline ABP 4 & BETAG & 1994Q1 & & 3.5 PCl \\
\hline ABP 4 & BETAG & 1994Q1 & & 6.2 PCL \\
\hline ABP 4 & BETAG & 1994Q1 & $\mathrm{J}$ & $1.53 \mathrm{PCL}$ \\
\hline ABP 4 & BETAG & 1994Q1 & $\mathrm{J}$ & $1.63 \mathrm{PCl}$ \\
\hline ABP 4 & BETAG & $1994 Q 3$ & UI & $0.837 \mathrm{PCl}$ \\
\hline ABP 4 & BETAG & 1995Q1 & UI & $1.22 \mathrm{PCL}$ \\
\hline ABP 4 & BETAG & 1996Q1 & & $3.2 \mathrm{PCl}$ \\
\hline ABP 4 & BETAG & 1996Q1 & $J$ & $1.92 \mathrm{PCl}$ \\
\hline ABP 4 & BETAG & 1996Q1 & $\mathrm{J}$ & $2.08 \mathrm{PCl}$ \\
\hline ABP 4 & BETAG & 1996Q1 & UI & $0.946 \mathrm{PCL}$ \\
\hline ABP 4 & BETAG & 1996Q1 & UI & $1.59 \mathrm{PCl}$ \\
\hline ABP 4 & BETAG & 199602 & & $0.783 \mathrm{PCl}$ \\
\hline ABP 4 & BETAG & 1997Q4 & & $4.12 \mathrm{PCl}$ \\
\hline $\mathrm{ABP} 4$ & BETAG & $1998 Q 3$ & & 2.69 PCl \\
\hline ABP 4 & BETAG & 1999Q3 & & $2.56 \mathrm{P}$ \\
\hline ABP 4 & BETAG & 1999Q3 & & $4.23 \mathrm{~F}$ \\
\hline
\end{tabular}

Nonvolatile beta in ABP 4
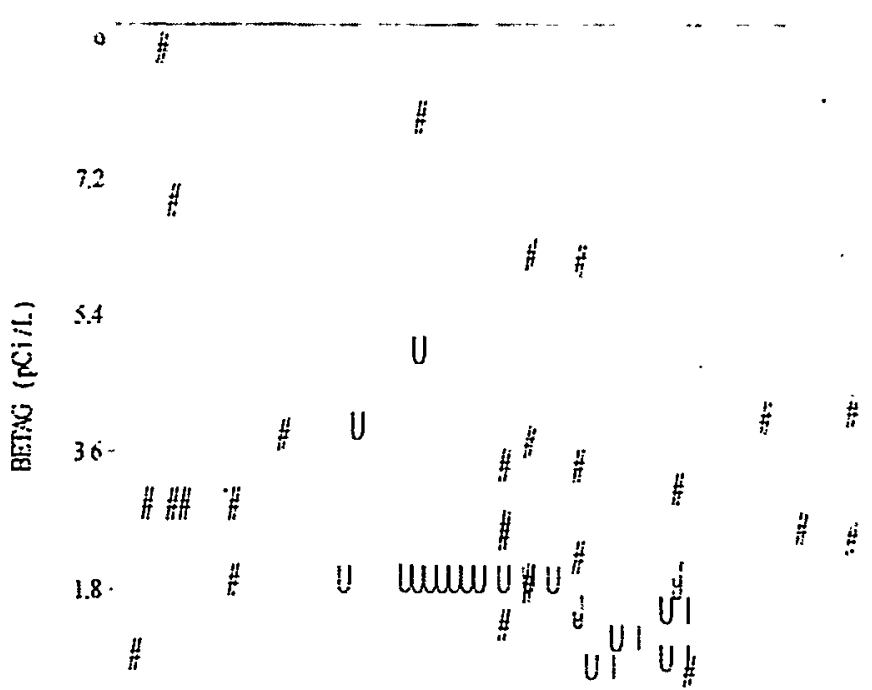

0 -

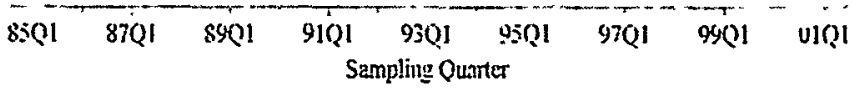
(Plot syinhol: Resqual if exisls or t) 
Cobalt, total recoverable in ABP 4

Data Retrieved by AWSA v1 from GIMS on 09NOV99 at 15:50

\begin{tabular}{llll} 
Well & Analyte & \multicolumn{2}{c}{ Sample_Q Res } \\
& & & \\
ABP 4 & COTOT & $1994 Q 3$ & $\mathrm{U}$ \\
ABP 4 & COTOT & $1996 Q 1$ & $\mathrm{~J}$ \\
ABP 4 & COTOT & $1996 Q 1$ & $\mathrm{U}$ \\
ABP 4 & COTOT & $1996 Q 1$ & $\mathrm{U}$ \\
ABP 4 & COTOT & $1996 Q 1$ & $\mathrm{UJ}$ \\
ABP 4 & COTOT & $1996 Q 1$ & $\mathrm{UJ}$ \\
ABP 4 & COTOT & $1996 \mathrm{2}$ & $\mathrm{U}$ \\
ABP 4 & COTOT & $1997 Q 4$ & $\mathrm{U}$ \\
ABP 4 & COTOT & $1998 Q 3$ & $\mathrm{~J}$ \\
ABP 4 & COTOT & $1999 Q 3$ & $\mathrm{U}$ \\
ABP 4 & COTOT & $1999 Q 3$ & $\mathrm{U}$
\end{tabular}

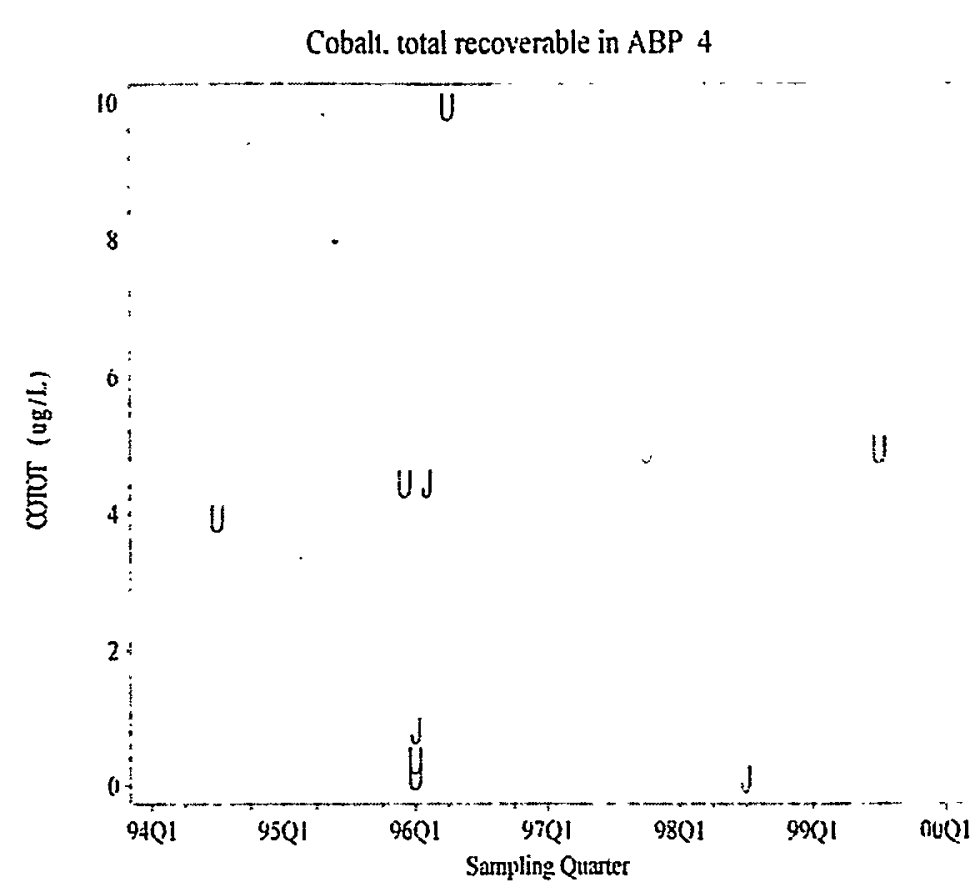

(Plot:symbol: Rexpual if exists or it) 
Copper, total recoverable in ABP 4

Data Retrieved by AWSA v1 from GIMS on 09NOV99 at 15:50

\begin{tabular}{|c|c|c|c|c|}
\hline Well & Analyte & Sample_c & Res_Qual & Result \\
\hline ABP 4 & СUTOT & $1994 Q 3$ & $u$ & 4 UGL \\
\hline $\mathrm{ABP} 4$ & CUTOT & 1996Q1 & & 19.8 UGL \\
\hline ABP 4 & CUTOT & 1996Q1 & $J$ & 1.68 UGL \\
\hline $\mathrm{ABP}_{4}$ & CUTOT & 1996Q1 & J & 2.1 UGL \\
\hline ABP 4 & CUTOT & $1996 Q 1$ & $U$ & 15 UGL \\
\hline ABP 4 & cUToT & 1996Q1 & U & 15 UGL \\
\hline ABP 4 & CUTOT & 1996Q2 & U & 25 UGL \\
\hline$A B P 4$ & СUTOT & $1997 Q 4$ & & 13.3 UGL \\
\hline ABP 4 & CUTOT & 1998Q3 & $U$ & 0.76 UGL \\
\hline ABP 4 & CUTOT & 199903 & $\mathbf{J}$ & 1.31 UGL \\
\hline ABP 4 & CUTOT & 1999Q3 & $J$ & 1.96 UGL \\
\hline
\end{tabular}
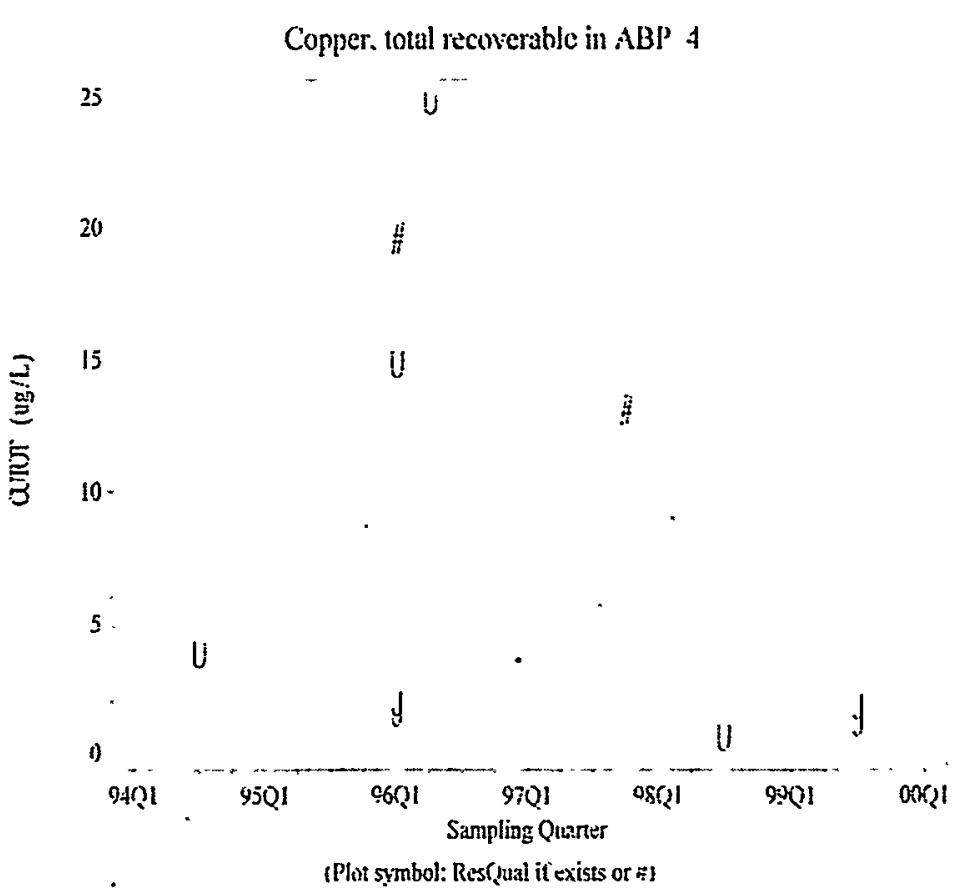
Cyanide in ABP 4

Data Retrieved by AWSA v1 from GIMS on 09NOV99 at 15:50

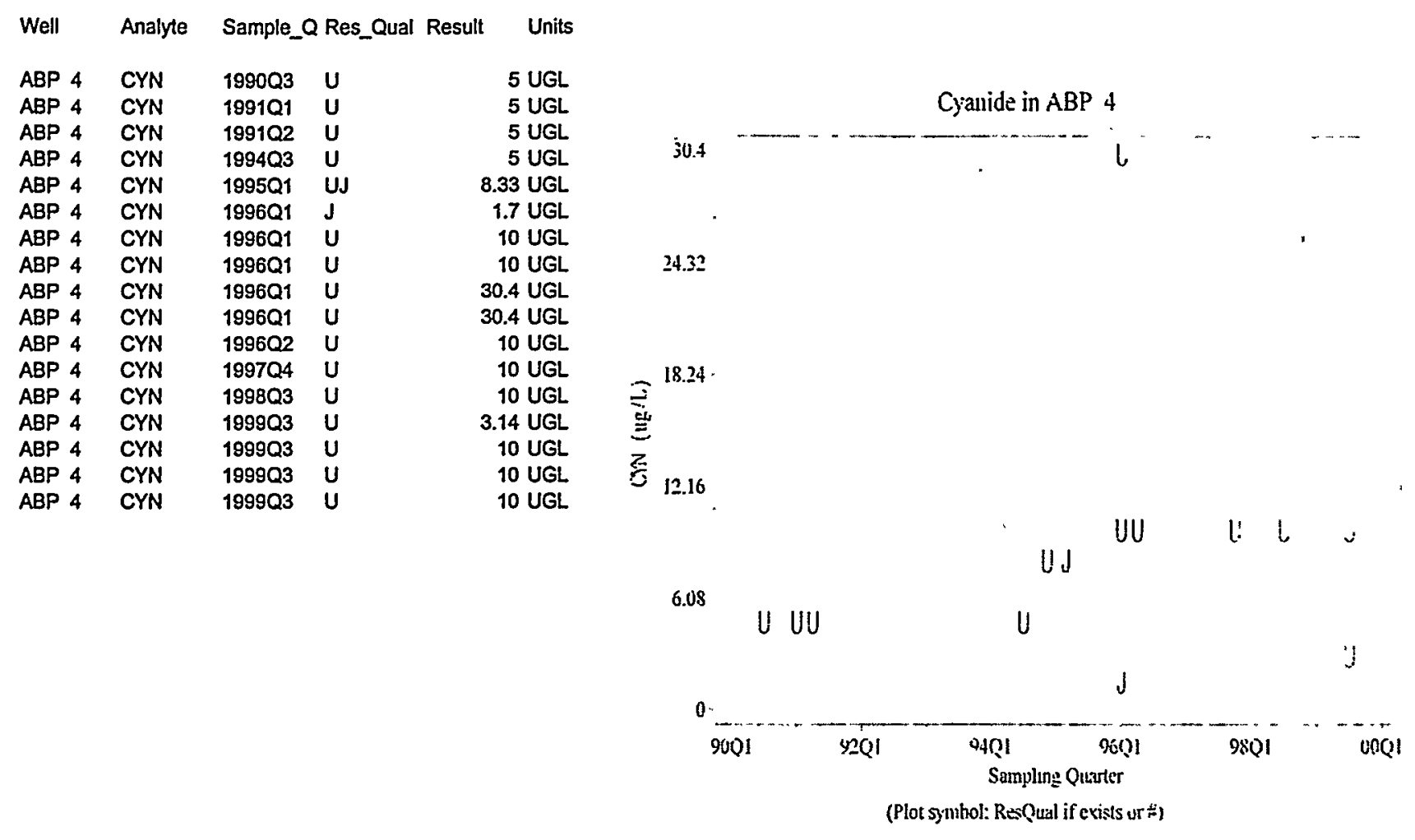


Fluoride in ABP 4

Data Retrieved by AWSA v1 from GIMS on 09NOV99 at 15:50

\begin{tabular}{|c|c|c|c|c|c|c|}
\hline Well & & Analyte & Sample_ & Res_Qual & Result & Units \\
\hline$A B P$ & 4 & $\boldsymbol{F}$ & 1985Q1 & & & $190 \mathrm{UGL}$ \\
\hline $\mathrm{ABP}$ & 4 & $F$ & $1985 Q 2$ & & & 80 UGL \\
\hline ABP & 4 & $F$ & $1985 Q 3$ & & & $100 \mathrm{UGL}$ \\
\hline$A B P$ & 4 & $F$ & 1985Q4 & & & 60 UGL \\
\hline$A B P$ & 4 & $\mathbf{F}$ & 1985Q4 & & 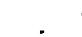 & 100 UGL \\
\hline$A B P$ & 4 & $F$ & 1987Q1 & & & 100 UGL \\
\hline$A B P$ & 4 & $\mathbf{F}$ & 1987Q1 & & & 100 UGL \\
\hline ABP & 4 & $\mathbf{F}$ & 198902 & U & & 100 UGL \\
\hline$A B P$ & 4 & $\mathbf{F}$ & $1989 Q 3$ & $U$ & & 250 UGL \\
\hline $\mathrm{ABP}$ & 4 & $\mathbf{F}$ & $1980 Q 3$ & $\mathbf{U}$ & & 100 UGL \\
\hline $\mathrm{ABP}$ & 4 & $F$ & 199004 & $U$ & & 100 UGL \\
\hline $\mathrm{ABP}$ & 4 & $\boldsymbol{F}$ & 199004 & $\mathbf{U}$ & & 100 UGL \\
\hline$A B P$ & 4 & $F$ & 1990Q4 & $U$ & & 250 UGL \\
\hline ABP & 4 & $\boldsymbol{F}$ & 199004 & $\mathbf{U}$ & & 250 UGL \\
\hline$A B P$ & 4 & $\boldsymbol{F}$ & 1991Q1 & U & & 100 UGL \\
\hline$A B P$ & 4 & $\mathbf{F}$ & 1991Q2 & U & & $100 \mathrm{UGL}$ \\
\hline$A B P$ & 4 & $F$ & 1991Q3 & U & & 100 UGL \\
\hline$A B P$ & 4 & $\mathbf{F}$ & $1991 Q 4$ & U & & 100 UGL \\
\hline$A B P$ & 4 & $\mathbf{F}$ & 1992Q1 & $\mathrm{U}$ & & 100 UGL \\
\hline$A B P$ & 4 & $\mathbf{F}$ & 1992Q1 & $u$ & & 100 UGL \\
\hline ABP & 4 & $F$ & 199301 & $\mathbf{U}$ & & 100 UGL \\
\hline$A B P$ & 4 & $F$ & 1993Q1 & $\mathbf{U}$ & & $100 \mathrm{UGL}$ \\
\hline ABP & 4 & $F$ & 1993Q1 & $\mathbf{U}$ & & 100 UGL \\
\hline ABP & 4 & $\boldsymbol{F}$ & 1993Q1 & $\mathbf{U}$ & & 100 UGL \\
\hline ABP & 4 & $F$ & 1997Q4 & $\mathrm{U}$ & & $50 \mathrm{UGL}$ \\
\hline$A B P$ & 4 & $F$ & 1998Q3 & $U$ & & 50 UGL \\
\hline ABP & 4 & $F$ & 1998Q3 & U & & 50 UGL \\
\hline ABP & 4 & $F$ & $1999 Q 3$ & $\mathrm{U}$ & & 50 UGL \\
\hline ABP & 4 & $\boldsymbol{F}$ & 199903 & $U$ & & 50 UGL \\
\hline
\end{tabular}

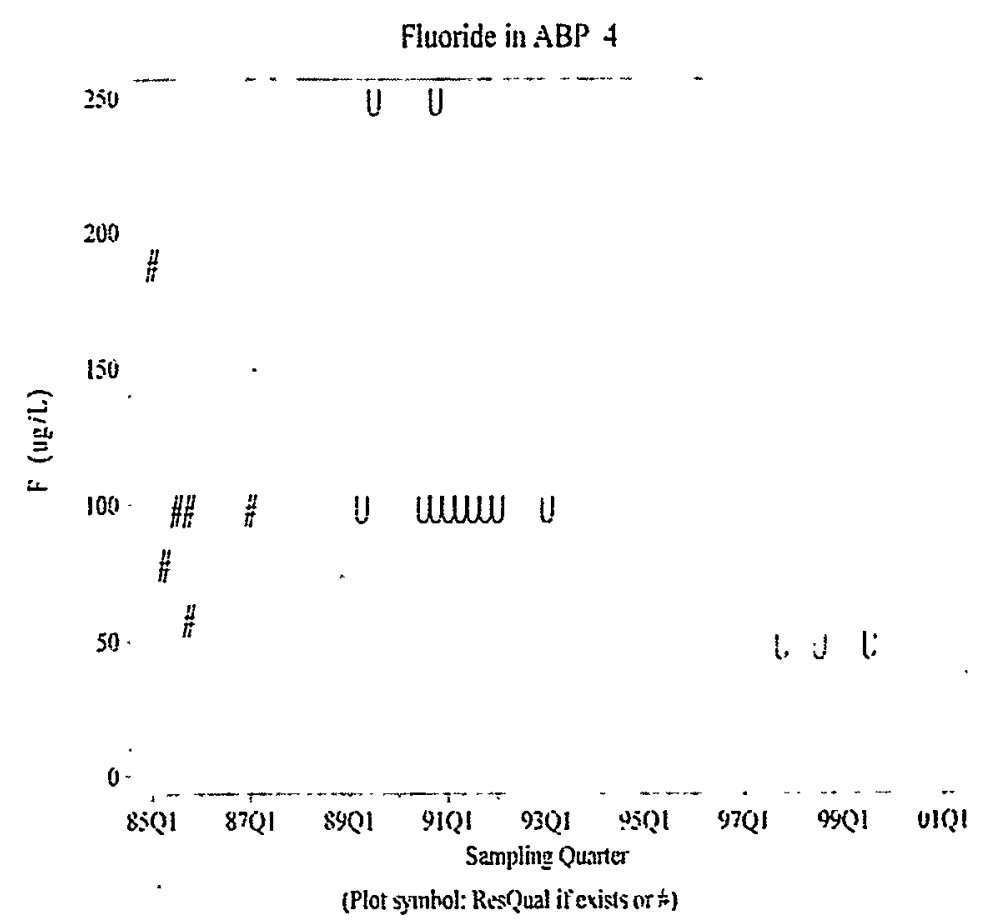


Mercury, total recoverable in ABP 4

Data Retrieved by AWSA v1 from GIMS on 09NOV99 at 15:50

\begin{tabular}{llll} 
Well & Analyte & \multicolumn{2}{c}{ Sample_Q Res_Q } \\
& & & \\
ABP 4 & HGTOT & $1992 Q 4$ & $U$ \\
ABP 4 & HGTOT & $1993 Q 1$ & $U$ \\
ABP 4 & HGTOT & $1993 Q 1$ & $U$ \\
ABP 4 & HGTOT & $1993 Q 1$ & $U$ \\
ABP 4 & HGTOT & $1993 Q 1$ & $U$ \\
ABP 4 & HGTOT & $1993 Q 1$ & $U$ \\
ABP 4 & HGTOT & $1995 Q 1$ & $U$ \\
ABP 4 & HGTOT & $1996 Q 1$ & $U$ \\
ABP 4 & HGTOT & $1996 Q 1$ & $U$ \\
ABP 4 & HGTOT & $1996 Q 1$ & $U$ \\
ABP 4 & HGTOT & $1996 Q 1$ & $U$ \\
ABP 4 & HGTOT & $1996 Q 1$ & $U$ \\
ABP 4 & HGTOT & $1996 Q 2$ & $U$ \\
ABP 4 & HGTOT & $1997 Q 4$ & $U$ \\
ABP 4 & HGTOT & $1998 Q 3$ & $U$ \\
ABP 4 & HGTOT & $1999 Q 3$ & $U$ \\
ABP 4 & HGTOT & $1999 Q 3$ & $U$
\end{tabular}

Units

0.2 UGL 0.2 UGL

0.2 UGL

0.2 UGL

0.2 UGL

0.2 UGL

0.333 UGL

0.2 UGL

0.2 UGL

0.7 UGL

0.7 UGL

0.7 UGL

0.2 UGL

0.2 UGL

0.2 UGL

0.2 UGL

0.2 UGL
Mercury, total recoverable in ABP 4

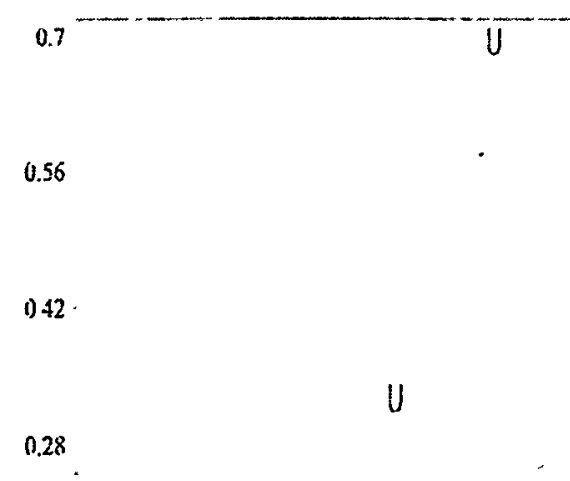

UU UJ $\quad$ U

0.14 -

0 .

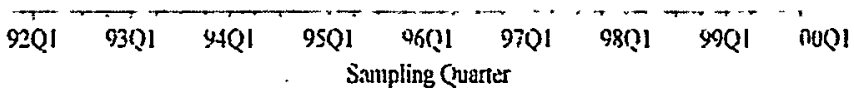

(Plot şmbill. Resuual if exists or ä) 
Nickel, total recoverable in ABP 4

Data Retrieved by AWSA v1 from GIMS on 09NOV99 at 15:50

\begin{tabular}{llll} 
Well & Analyte & \multicolumn{2}{c}{ Sample_Q Res } \\
& & & \\
ABP 4 & NITOT & $1992 Q 4$ & $U$ \\
ABP 4 & NITOT & $1993 Q 1$ & $U$ \\
ABP 4 & NITOT & $1993 Q 1$ & $U$ \\
ABP 4 & NITOT & $1993 Q 1$ & $U$ \\
ABP 4 & NITOT & $1993 Q 1$ & $U$ \\
ABP 4 & NITOT & $1993 Q 1$ & $U$ \\
ABP 4 & NITOT & $1994 Q 3$ & $U$ \\
ABP 4 & NITOT & $1995 Q 1$ & $U$ \\
ABP 4 & NITOT & $1996 Q 1$ & $U$ \\
ABP 4 & NITOT & $1996 Q 1$ & $U$ \\
ABP 4 & NITOT & $1996 Q 1$ & $U$ \\
ABP 4 & NITOT & $1996 Q 1$ & $U$ \\
ABP 4 & NITOT & $1996 Q 1$ & $U$ \\
ABP 4 & NITOT & $1996 Q 2$ & $U$ \\
ABP 4 & NITOT & $1997 Q 4$ & $U$ \\
ABP 4 & NITOT & $1998 Q 3$ & \\
ABP 4 & NITOT & $1999 Q 3$ & $J$ \\
ABP 4 & NITOT & $1999 Q 3$ & $U$
\end{tabular}

Units

4 UGL 4 UGL 4 UGL 4 UGL 4 UGL 4 UGL 4 UGL $6.67 \mathrm{UGL}$ $10 \mathrm{UGL}$

10 UGL 26 UGL 26 UGL 26 UGL 25 UGL 5 UGL 0.914 UGL 2.63 UGL

5 UGL
Nickel, total recoveruble in ABP 4

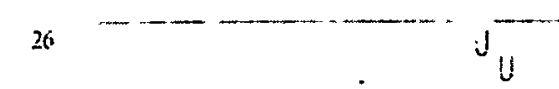

205

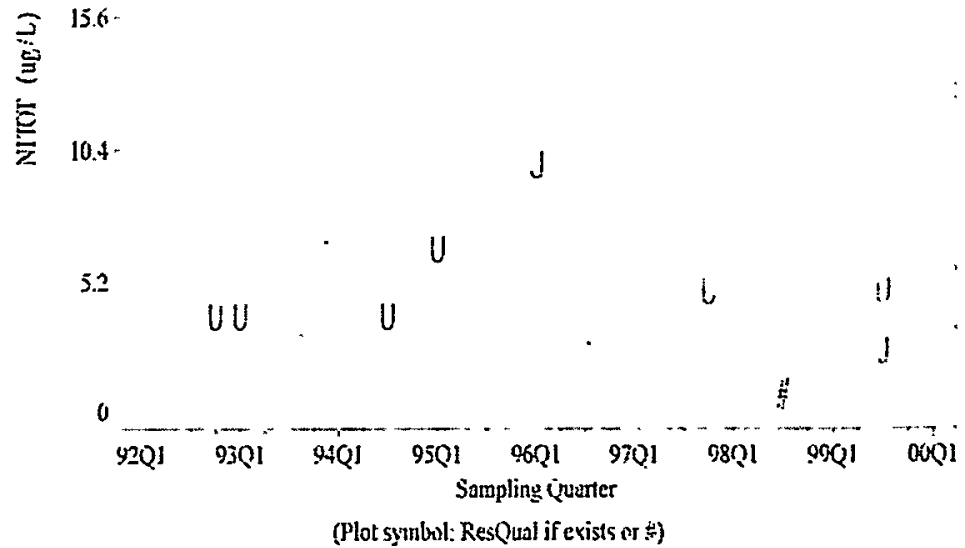


Nitrate-nitrite as nitrogen in $A B P 4$

Data Retrieved by AWSA v1 from GIMS on 09NOV99 at 15:50

$\begin{array}{lllr}\text { Well } & \text { Analyte } & \text { Sample_Q Res_Qual Result } & \text { Units } \\ \text { ABP 4 } & \text { NO3NO2 } & 1989 Q 2 & 770 \text { UGL } \\ \text { ABP 4 } & \text { NO3NO2 } & 1990 Q 3 & 1230 \text { UGL } \\ \text { ABP 4 } & \text { NO3NO2 } & 1990 Q 4 & 1230 \text { UGL } \\ \text { ABP 4 } & \text { NO3NO2 } & 1990 Q 4 & 1300 \text { UGL } \\ \text { ABP 4 } & \text { NO3NO2 } & 1991 Q 1 & 1330 \text { UGL } \\ \text { ABP 4 } & \text { NO3NO2 } & 1991 Q 3 & 1100 \text { UGL } \\ \text { ABP 4 } & \text { NO3NO2 } & 1991 Q 4 & 890 \text { UGL } \\ \text { ABP 4 } & \text { NO3NO2 } & 1992 Q 1 & 800 \text { UGL } \\ \text { ABP 4 } & \text { NO3NO2 } & 1993 Q 1 & 620 \text { UGL } \\ \text { ABP 4 } & \text { NO3NO2 } & 1993 Q 1 & 630 \text { UGL } \\ \text { ABP 4 } & \text { NO3NO2 } & 1997 Q 4 & 250 \text { UGL } \\ \text { ABP 4 } & \text { NO3NO2 } & 1998 Q 3 & 280 \text { UGL } \\ \text { ABP 4 } & \text { NO3NO2 } & 1999 Q 3 & 250 \text { UGL } \\ \text { ABP 4 } & \text { NO3NO2 } & 1999 Q 3 & 250 \text { UGL } \\ \text { ABP 4 } & \text { NO3NO2 } & 1999 Q 3 & 250 \text { UGL }\end{array}$

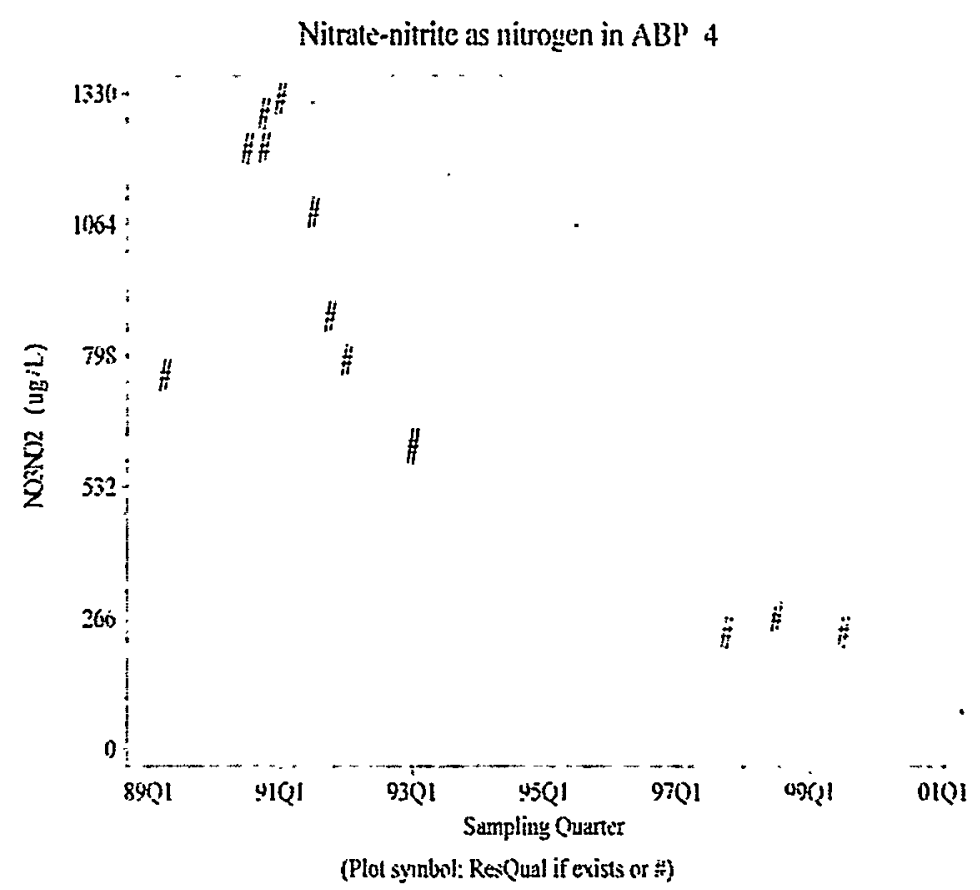


Selenium, total recoverable in ABP 4

Data Retrieved by AWSA v1 from GIMS on 09NOV99 at 15:50

\begin{tabular}{llllr} 
Well & Analyte & \multicolumn{2}{c}{ Sample_Q Res_Qual Result } & Units \\
& & & & \\
ABP 4 & SETOT & $1993 Q 1$ & $U$ & 2 UGL \\
ABP 4 & SETOT & $1993 Q 1$ & $U$ & 2 UGL \\
ABP 4 & SETOT & $1993 Q 1$ & $U$ & 2 UGL \\
ABP 4 & SETOT & $1993 Q 1$ & $U$ & 2 UGL \\
ABP 4 & SETOT & $1993 Q 1$ & $U$ & 2 UGL \\
ABP 4 & SETOT & $1993 Q 1$ & $U$ & 2 UGL \\
ABP 4 & SETOT & $1994 Q 3$ & $U$ & 2 UGL \\
ABP 4 & SETOT & $1995 Q 1$ & $U$ & 3.33 UGL \\
ABP 4 & SETOT & $1996 Q 1$ & J & 1.5 UGL \\
ABP 4 & SETOT & $1996 Q 1$ & $U$ & 5 UGL \\
ABP 4 & SETOT & $1996 Q 1$ & $U$ & 66 UGL \\
ABP 4 & SETOT & $1996 Q 1$ & $U$ & 66 UGL \\
ABP 4 & SETOT & $1996 Q 1$ & $U$ & 66 UGL \\
ABP 4 & SETOT & $1996 Q 2$ & UJ & 2 UGL \\
ABP 4 & SETOT & $1997 Q 4$ & $U$ & 5 UGL \\
ABP 4 & SETOT & $1998 Q 3$ & $U$ & 5 UGL \\
ABP 4 & SETOT & $1999 Q 3$ & $U$ & 5 UGL \\
ABP 4 & SETOT & $1999 Q 3$ & $U$ & 5 UGL \\
& & & &
\end{tabular}

Selenium, total recoverable in ABP 4

60

U
52.5

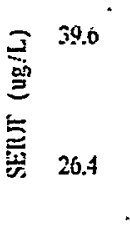

13.2

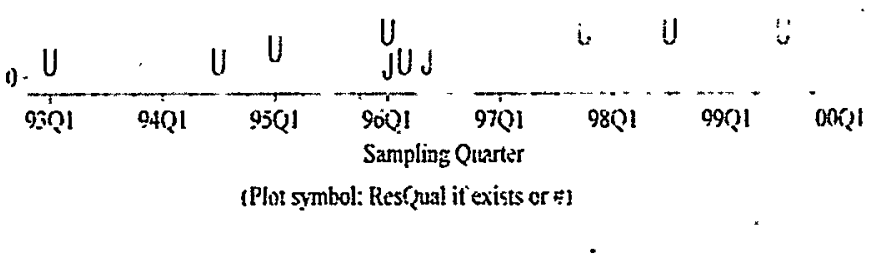


trans-1,2-Dichloroethylene in ABP 4

Data Retrieved by AWSA v1 from GIMS on 09NOV99 at 15:50

\begin{tabular}{|c|c|c|c|c|c|c|}
\hline Well & & Analyte & Sample_C & Res_Qual & Result & Units \\
\hline$A B P$ & 4 & T12DCE & $1985 Q 4$ & & & 5 UGL \\
\hline$A B P$ & 4 & T12DCE & 1985Q4 & & & 5 UGL \\
\hline ABP & 4 & T12DCE & 1987Q1 & & & 5 UGL \\
\hline ABP 4 & 4 & T12DCE & $1988 Q 3$ & & & $1 \mathrm{UGL}$ \\
\hline$A B P$ & 4 & T12DCE & 1988Q4 & $\mathbf{U}$ & & $1 \mathrm{UGL}$ \\
\hline ABP 4 & 4 & T12DCE & $1988 Q 4$ & $\mathbf{U}$ & & $1 \mathrm{UGL}$ \\
\hline ABP 4 & 4 & T12DCE & 1989Q1 & $\mathbf{U}$ & & $1 \mathrm{UGL}$ \\
\hline$A B P$ & 4 & T12DCE & $1989 Q 2$ & $U$ & & $1 \mathrm{UGL}$ \\
\hline$A B P 4$ & 4 & T12DCE & 198903 & $\mathbf{U}$ & & $1 \mathrm{UGL}$ \\
\hline$A B P$ & 4 & T12DCE & $1989 Q 3$ & U & & 1 UGL \\
\hline ABP 4 & 4 & T12DCE & 1989Q3 & $u$ & & $1 \mathrm{UGL}$ \\
\hline$A B P 4$ & 4 & T12DCE & $1989 Q 3$ & $\mathbf{U}$ & & 1 UGL \\
\hline$A B P$ & 4 & T12DCE & 198903 & $\mathbf{U}$ & & 5 UGL \\
\hline$A B P$ & 4 & T120CE & 198903 & $\mathbf{U}$ & & 5 UGL \\
\hline$A B P$ & 4 & T12DCE & $1989 Q 4$ & $\mathbf{U}$ & & 1 UGL \\
\hline$A B P$ & 4 & T12DCE & 1990Q1 & U & . & 1 UGL. \\
\hline$A B P$ & 4 & T12DCE & 1990Q1 & U & & 1 UGL \\
\hline ABP & 4 & T12DCE & 1990Q2 & U & & 1 UGL \\
\hline$A B P 4$ & 4 & T12DCE & 1990Q3 & $\mathbf{U}$ & & $1 \mathrm{UGL}$ \\
\hline$A B P$ & 4 & T12DCE & 199003 & $\mathbf{U}$ & & 1 UGL \\
\hline ABP 4 & 4 & T12DCE & $1990 Q 4$ & $\mathbf{U}$ & & 1 UGL \\
\hline$A B P$ & 4 & T12DCE & 199004 & $\mathbf{U}$ & & $1 \mathrm{UGL}$ \\
\hline$A B P$ & 4 & T12DCE & $1990 Q 4$ & $\mathbf{U}$ & & $5 \cdot \mathrm{UGL}$ \\
\hline$A B P 4$ & 4 & T12DCE & 199004 & $\mathbf{U}$ & & 5 UGL \\
\hline$A B P$ & 4 & T12DCE & $1990 Q 4$ & $\mathbf{U}$ & & 5 UGL \\
\hline$A B P 4$ & 4 & T12DCE & 199004 & $\mathbf{U}$ & & 5 UGL \\
\hline$A B P$ & 4 & T12DCE & 1991Q1 & $\mathbf{U}$ & & 1 UGL \\
\hline$A B P$ & 4 & T12DCE & 1991Q2 & $\mathbf{U}$ & & 1 UGL \\
\hline ABP 4 & 4 & T12DCE & 1991Q3 & $\mathbf{U}$ & & 1 UGL \\
\hline$A B P$ & 4 & T12DCE & 1991Q4 & $\mathbf{U}$ & & 1 UGL \\
\hline$A B P$ & 4 & T12DCE & 1892Q1 & $\mathbf{U}$ & & 1 UGL \\
\hline ABP 4 & 4 & T12DCE & 199202 & $\mathbf{U}$ & & $1 \mathrm{UGL}$ \\
\hline$A B P$ & 4 & T12DCE & 1992Q3 & $\mathbf{U}$ & & $1 \mathrm{UGL}$ \\
\hline ABP 4 & 4 & T12DCE & 1992Q3 & $\mathbf{U}$ & & $1 \mathrm{UGL}$ \\
\hline $\mathrm{ABP} 4$ & 4 & T12DCE & 1992Q3 & $\mathbf{U}$ & & 1 UGL \\
\hline$A B P$ & 4 & T12DCE & 1992Q3 & $\mathbf{U}$ & & 5 UGL \\
\hline$A B P$ & 4 & T12DCE & 1992Q3 & $\mathbf{u}$ & & 5 UGL \\
\hline$A B P$ & 4 & T12DCE & 1992Q4 & $\mathbf{U}$ & & $1 \mathrm{UGL}$ \\
\hline$A B P$ & 4 & T12DCE & 1993Q1 & $\mathbf{U}$ & & 1 UGL \\
\hline$A B P$ & 4 & T12DCE & 1993Q1 & $\mathbf{U}$ & & 1 UGL \\
\hline$A B P$ & 4 & T12DCE & 1993Q1 & $\mathbf{U}$ & & 5 UGL \\
\hline$A B P 4$ & 4 & T12DCE & 1993Q1 & $\mathbf{U}$ & & 5 UGL \\
\hline ABP 4 & 4 & T12DCE & 1993Q2 & $\mathbf{U}$ & & 1 UGL \\
\hline$A B P 4$ & 4 & T12DCE & 1993Q3 & UJ & & $1 \mathrm{UGL}$ \\
\hline$A B P$ & 4 & T12DCE & $1993 Q 4$ & $\mathbf{U}$ & & 1 UGL \\
\hline $\mathrm{ABP}$ & 4 & T12DCE & 199304 & U & & $1 \mathrm{UGL}$ \\
\hline$A B P$ & 4 & T12DCE & 1994Q1 & U & & 1 UGL \\
\hline$A B P$ & 4 & T12DCE & 1994Q1 & $\mathrm{U}$ & & $1 \mathrm{UGL}$ \\
\hline$A B P$ & 4 & T12DCE & 1994Q2 & $\mathrm{U}$ & & 1 UGL \\
\hline$A B P 4$ & 4 & T12DCE & 1994Q3 & $\mathbf{U}$ & & $1 \mathrm{UGL}$ \\
\hline$A B P$ & 4 & T12DCE & 1995Q1 & $\mathbf{U}$ & & 1.67 UGL \\
\hline$A B P 4$ & 4 & T12DCE & 1997Q4 & $\mathbf{U}$ & & $1 \mathrm{UGL}$ \\
\hline$A B P$ & 4 & T12DCE & $1998 Q 3$ & U & & 1 UGL \\
\hline$A B P$ & 4 & T12DCE & $1999 Q 3$ & $\mathbf{U}$ & & 1 UGL \\
\hline ABP 4 & 4 & T12DCE & 199903 & $U$ & & $1 \mathrm{UGL}$ \\
\hline
\end{tabular}

Irans-1.2-Dichloreethylene in $A B P+$

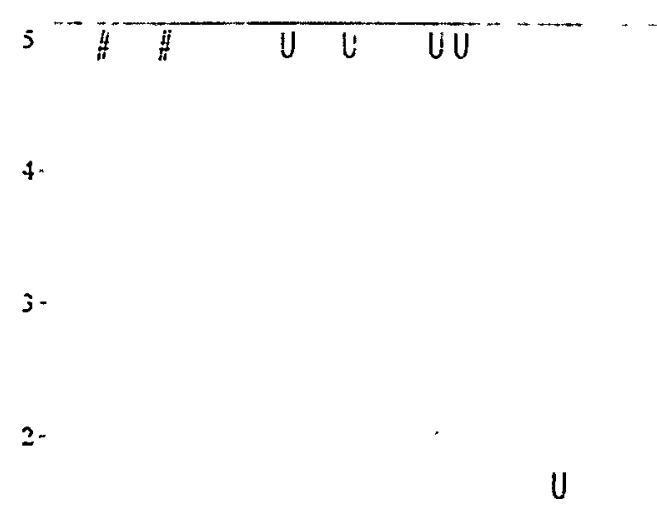

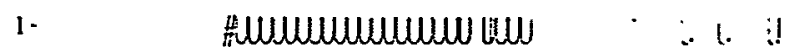

0 -

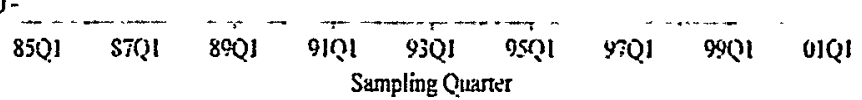
(Plot ssynbol: Rernual it exists or t) 
1,1,2,2-Tetrachloroethane in ABP 4

Data Retrieved by AWSA v1 from GIMS on 09NOV99 at 15:50

\begin{tabular}{|c|c|c|c|c|c|}
\hline Well & Analyte & Sample_ & Res_Qual & Result & Units \\
\hline$A B P 4$ & TCLEA & $1985 Q 4$ & & & 10 UGL \\
\hline$A B P 4$ & TCLEA & 198504 & & & 10 UGL \\
\hline$A B P 4$ & TCLEA & 1887Q1 & & & 10 UGL \\
\hline $\mathrm{ABP} 4$ & TCLEA & $1989 Q 3$ & $\mathbf{U}$ & & 1 UGL \\
\hline$A B P 4$ & TCLEA & 198903 & $U$ & . & $1 \mathrm{UGL}$ \\
\hline $\mathrm{ABP} 4$ & TCLEA & 198903 & $\mathbf{U}$ & & 5 UGL \\
\hline ABP 4 & TCLEA & $1989 Q 3$ & $\mathbf{U}$ & & 5 UGL \\
\hline$A B P 4$ & TCLEA & 199003 & U & & 1 UGL \\
\hline ABP 4 & TCLEA & $1990 Q 4$ & $U$ & & 5 UGL \\
\hline $\mathrm{ABP} 4$ & TCLEA & $1990 Q 4$ & $\mathbf{u}$ & & 5 UGL \\
\hline$A B P 4$ & TCLEA & 199004 & $\mathbf{U}$ & & 5 UGL \\
\hline$A B P 4$ & TCLEA & $1990 Q 4$ & $\mathbf{U}$ & & 5 UGL \\
\hline $\mathrm{ABP} 4$ & TCLEA & 1991Q1 & $\mathbf{U}$ & & 1 UGL \\
\hline$A B P 4$ & TCLEA & 1991Q2 & $u$ & & 1 UGL \\
\hline$A B P 4$ & TCLEA & 1991Q3 & U & & 1 UGL \\
\hline ABP 4 & TCLEA & 1891Q4 & $\mathbf{U}$ & & 1 UGL \\
\hline$A B P 4$ & TCLEA & 1992Q1 & $\mathbf{U}$ & & $1 \mathrm{UGL}$ \\
\hline$A B P 4$ & TCLEA & 1992Q2 & $\mathbf{U}$ & & $1 \mathrm{UGL}$ \\
\hline ABP 4 & TCLEA & $1992 Q 3$ & $\mathbf{U}$ & & 1 UGL \\
\hline ABP 4 & TCLEA & $1992 Q 3$ & $\mathbf{U}$ & & $1 \mathrm{UGL}$ \\
\hline$A B P 4$ & TCLEA & 1992Q3 & $\mathbf{U}$ & & $1 \mathrm{UGL}$ \\
\hline$A B P 4$ & TCLEA & $1992 \mathrm{Q3}$ & $\mathbf{U}$ & & $5 \mathrm{UGL}$ \\
\hline$A B P 4$ & TCLEA & $1892 Q 3$ & $\mathbf{U}$ & & 5 UGL \\
\hline$A B P 4$ & TCLEA & $1992 Q 4$ & $\mathbf{U}$ & & $1 \mathrm{UGL}$ \\
\hline$A B P 4$ & TCLEA & 1993Q1 & $\mathbf{U}$ & & $1 \mathrm{UGL}$ \\
\hline$A B P 4$ & TCLEA & 1993Q1 & $\mathbf{U}$ & & $1 \mathrm{UGL}$ \\
\hline$A B P 4$ & TCLEA & 1993Q1 & $\mathbf{U}$ & & 5 UGL \\
\hline$A B P 4$ & TCLEA & 1993Q1 & $U$ & & 5 UGL \\
\hline$A B P 4$ & TCLEA & 199302 & $\mathbf{U}$ & & $1 \mathrm{UGL}$ \\
\hline$A B P 4$ & TCLEA & $1993 Q 3$ & UJ & & 1 UGL \\
\hline$A B P 4$ & TCLEA & 1993Q4 & $\mathbf{U}$ & & $1 \mathrm{UGL}$ \\
\hline$A B P 4$ & TCLEA & 199304 & $\mathbf{U}$ & & $1 \mathrm{UGL}$ \\
\hline ABP 4 & TCLEA & $1994 Q 1$ & $\mathrm{U}$ & & $1 \mathrm{UGL}$ \\
\hline$A B P 4$ & TCLEA & 1994Q1 & $\mathrm{U}$ & & 1 UGL \\
\hline$A B P 4$ & TCLEA & 1994Q2 & $\mathbf{U}$ & & 1 UGL \\
\hline$A B P 4$ & TCLEA & $1994 Q 3$ & $U$ & & 1 UGL \\
\hline$A B P 4$ & TCLEA & 1995Q1 & $U$ & & $1.67 \mathrm{UGL}$ \\
\hline$A B P 4$ & TCLEA & 1996Q1 & $u$ & & 2 UGL \\
\hline$A B P 4$ & TCLEA & 1996Q1 & $\mathrm{U}$ & & $2 U G L$ \\
\hline $\mathrm{ABP} 4$ & TCLEA & 1996Q1 & $\mathrm{U}$ & & 2 UGL \\
\hline$A B P 4$ & TCLEA & 1996Q1 & $\mathrm{U}$ & & 5 UGL \\
\hline$A B P 4$ & TCLEA & 1996Q1 & $\mathrm{U}$ & & 5 UGL \\
\hline$A B P 4$ & TCLEA & 199602 & $\mathbf{U}$ & & 2 UGL \\
\hline$A B P 4$ & TCLEA & $1997 Q 4$ & $\mathbf{U}$ & & $1 \mathrm{UGL}$ \\
\hline$A B P 4$ & TCLEA & 1998Q3 & $\mathbf{U}$ & & 1 UGL \\
\hline$A B P 4$ & TCLEA & $1999 Q 3$ & $U$ & & $1 \mathrm{UGL}$ \\
\hline$A B P 4$ & TCLEA & $1999 Q 3$ & $U$ & & 1 UGL \\
\hline
\end{tabular}

1.1,2.2-Tetrachloroethune in $A B P 4$

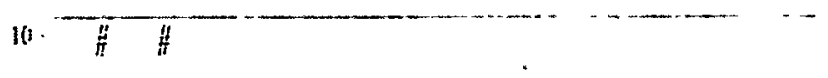

U U UU U

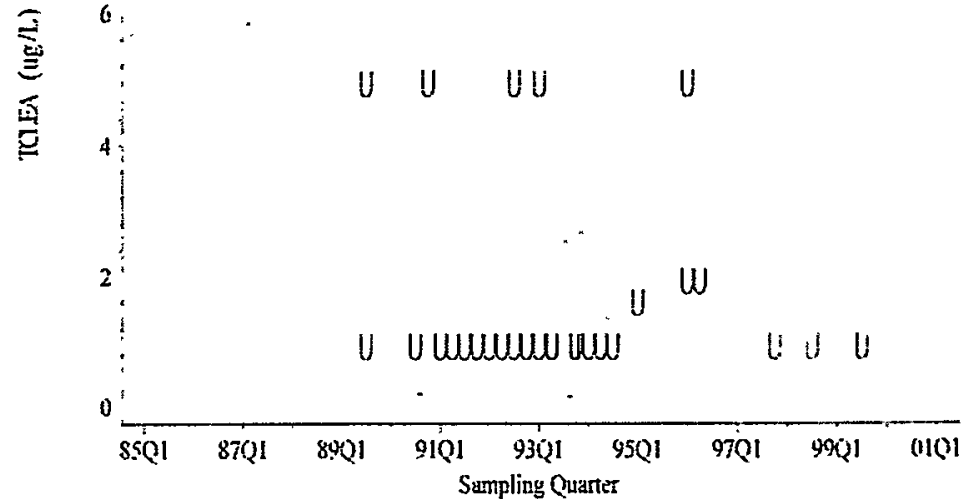

(Plol symbol: Res()ual if evists or :) 
Zinc, total recoverable in ABP 4

Data Retrieved by AWSA v1 from GIMS on 23NOV99 at 08:45

\begin{tabular}{llllr} 
Well & Analyte & \multicolumn{2}{c}{ Sample_Q Res_Qual Result } & Units \\
& & & & \\
ABP 4 & ZNTOT & $1994 Q 3$ & & 23.3 UGL \\
ABP 4 & ZNTOT & $1995 Q 1$ & $U$ & 3.33 UGL \\
ABP 4 & ZNTOT & $1996 Q 1$ & $\mathrm{~J}$ & 3.93 UGL \\
ABP 4 & ZNTOT & $1996 Q 1$ & $\mathrm{~J}$ & 9.12 UGL \\
ABP 4 & ZNTOT & $1996 Q 1$ & UJ & 5.6 UGL \\
ABP 4 & ZNTOT & $1996 Q 1$ & UJ & 7.1 UGL \\
ABP 4 & ZNTOT & $1996 Q 1$ & UJ & 53 UGL \\
ABP 4 & ZNTOT & $1996 Q 2$ & $U$ & 20 UGL \\
ABP 4 & ZNTOT & $1997 Q 4$ & & 30 UGL \\
ABP 4 & ZNTOT & $1998 Q 3$ & $U$ & 5 UGL \\
ABP 4 & ZNTOT & $1999 Q 3$ & $U$ & 3.4 UGL \\
ABP 4 & ZNTOT & $1999 Q 3$ & $U$ & 4.22 UGL
\end{tabular}

Zinc. total recoverable in ABP 4
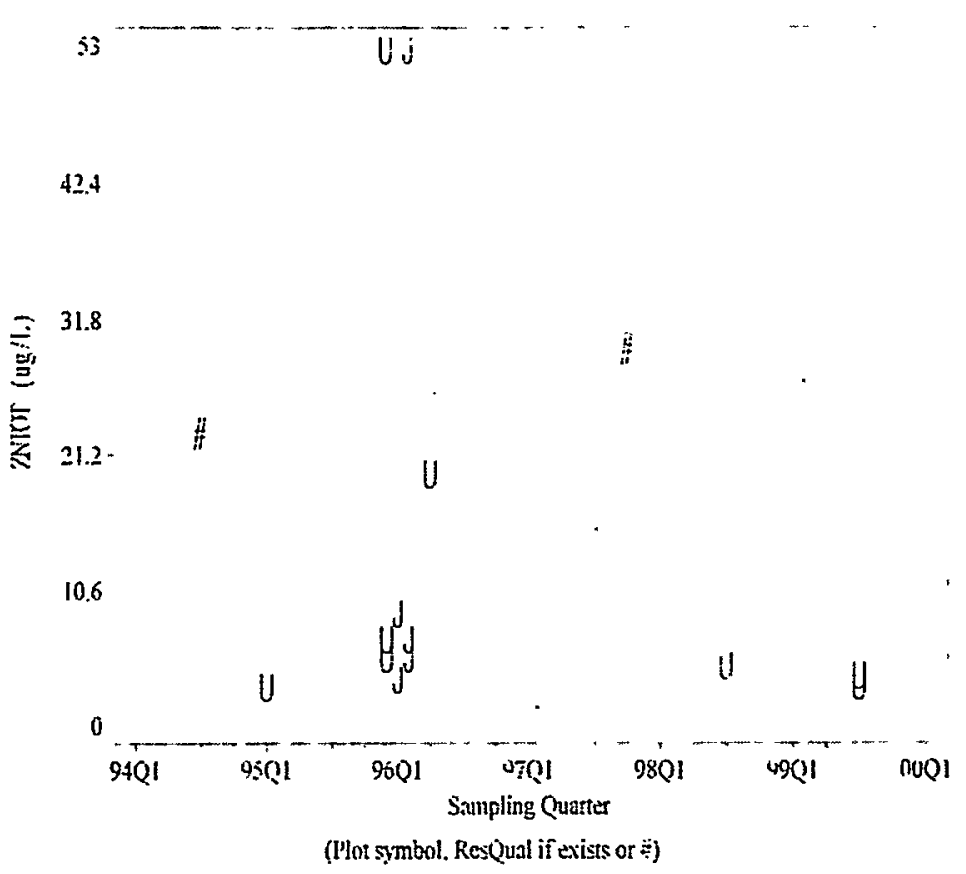
WELL ARP-3 DATA 
Data Retrieved by AWSA v1 from GIMS on 09NOV99 at 16:22

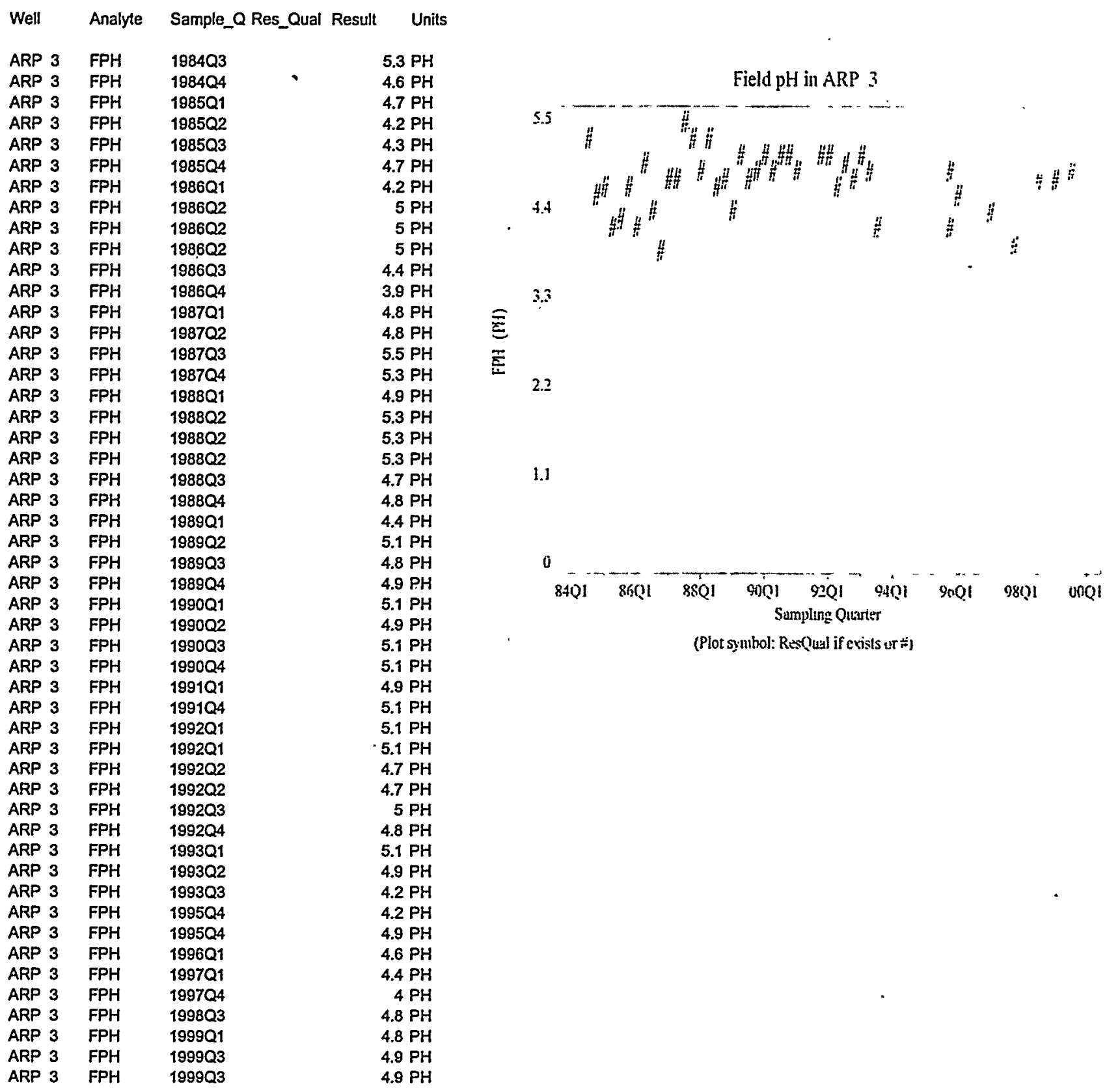


Specific Conductance in ARP 3

Data Retrieved by AWSA v1 from GIMS on 09NOV99 at 16:22

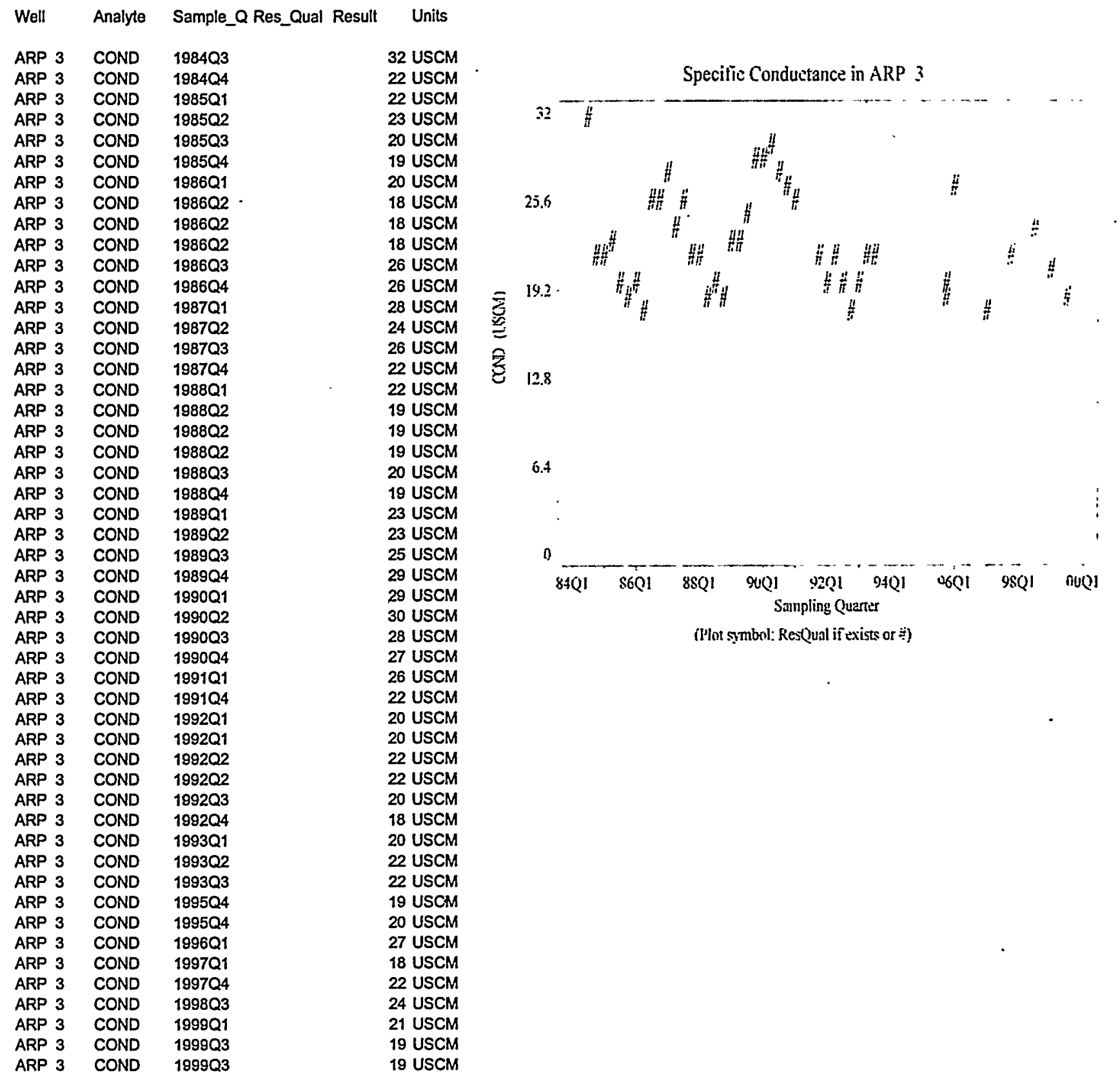


Calcium, total recoverable in ARP 3

Data Retrieved by AWSA v1 from GIMS on 09NOV99 at 15:50

Well Analyte Sample_Q Res_Qual Result Units

ARP $3 \quad$ CATOT 1993Q1 355 UGL

ARP 3 CATOT 1995Q4 441 UGL

ARP 3 CATOT 1995Q4 483 UGL

ARP 3 CATOT 1997Q4 373 UGL

ARP 3 CATOT $1998 Q 3 \quad 502$ UGL

ARP 3 CATOT $199903 \quad 454$ UGL
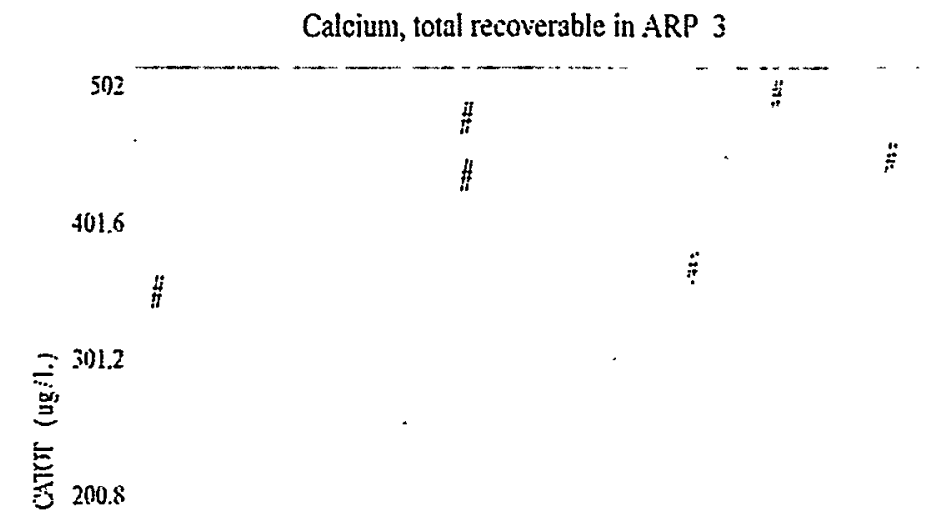

100.4

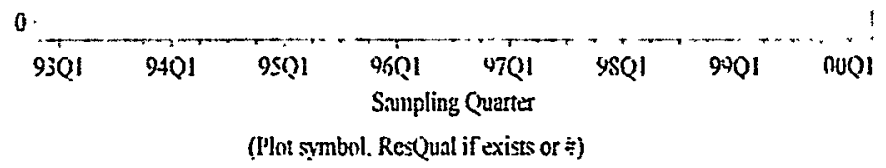


Potassium, total recoverable in ARP 3

Data Retrieved by AWSA v1 from GIMS on 09NOV99 at 15:50

\begin{tabular}{|c|c|c|c|c|c|}
\hline Well & Analyte & Sample & Res_Qual & Result & Units \\
\hline ARP 3 & КTOT & 1993Q1 & U & & 500 UGL \\
\hline ARP 3 & КтОТ & $1995 Q 4$ & J & & 149 UGL \\
\hline ARP 3 & КTOT & $1995 Q 4$ & $J$ & & 206 UGL \\
\hline ARP 3 & KTOT & 1997Q4 & & & 174 UGL \\
\hline ARP 3 & КтоT & 1998Q3 & & & 188 UGL \\
\hline & KTOT & $1999 Q 3$ & & & 187 UGL \\
\hline
\end{tabular}

Potassium, total recoversble in ARP 3

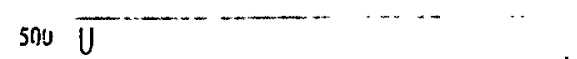

400

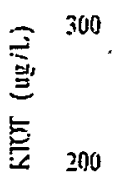

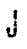

J

$\ddot{*} \quad \ddot{t}$

100

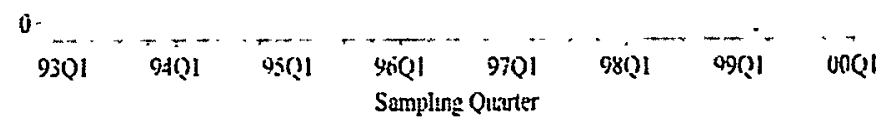

0 (Plot s) whol: ResQual if exisls ur ti) 
Sodium, total recoverable in ARP 3

Data Retrieved by AWSA v1 from GIMS on 09NOV99 at 15:50

Well Analyte Sample_QRes_Qual Result Units

ARP $3 \quad$ NATOT 1993 Q1 1970 UGL

ARP 3 NATOT 1995Q4 1770 UGL

ARP 3 NATOT 1995Q4 1790 UGL

ARP 3 NATOT 1997Q4 $2030 \mathrm{UGL}$

ARP 3 NATOT 1998Q3 $2440 \mathrm{UGL}$

ARP 3 NATOT 1999Q3 1810 UGL

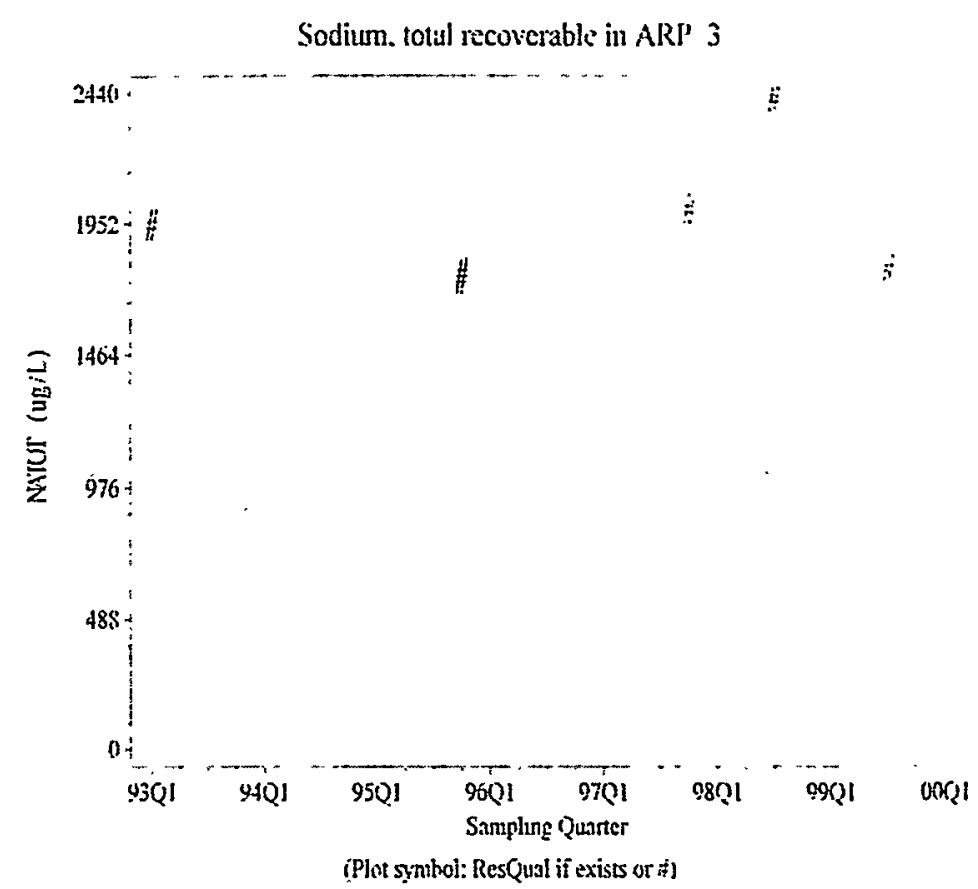


Chromium, total recoverable in ARP 3

Data Retrieved by AWSA v1 from GIMS on 09NOV99 at 15:50

\begin{tabular}{llrlr} 
Well & Analyte & \multicolumn{2}{c}{ Sample_Q Res_Qual Result } & Units \\
& & & & \\
ARP 3 & CRTOT & $1993 Q 1$ & U & 4 UGL \\
ARP 3 & CRTOT & $1995 Q 4$ & & 52.6 UGL \\
ARP 3 & CRTOT & 199504 & & 57 UGL \\
ARP 3 & CRTOT & $1995 Q 4$ & U & 3.02 UGL \\
ARP 3 & CRTOT & $1997 Q 1$ & & 19.7 UGL \\
ARP 3 & CRTOT & $1997 Q 4$ & J & 1.95 UGL \\
ARP 3 & CRTOT & $1998 Q 3$ & & 5.85 UGL \\
ARP 3 & CRTOT & $1999 Q 3$ & 9.37 UGL
\end{tabular}

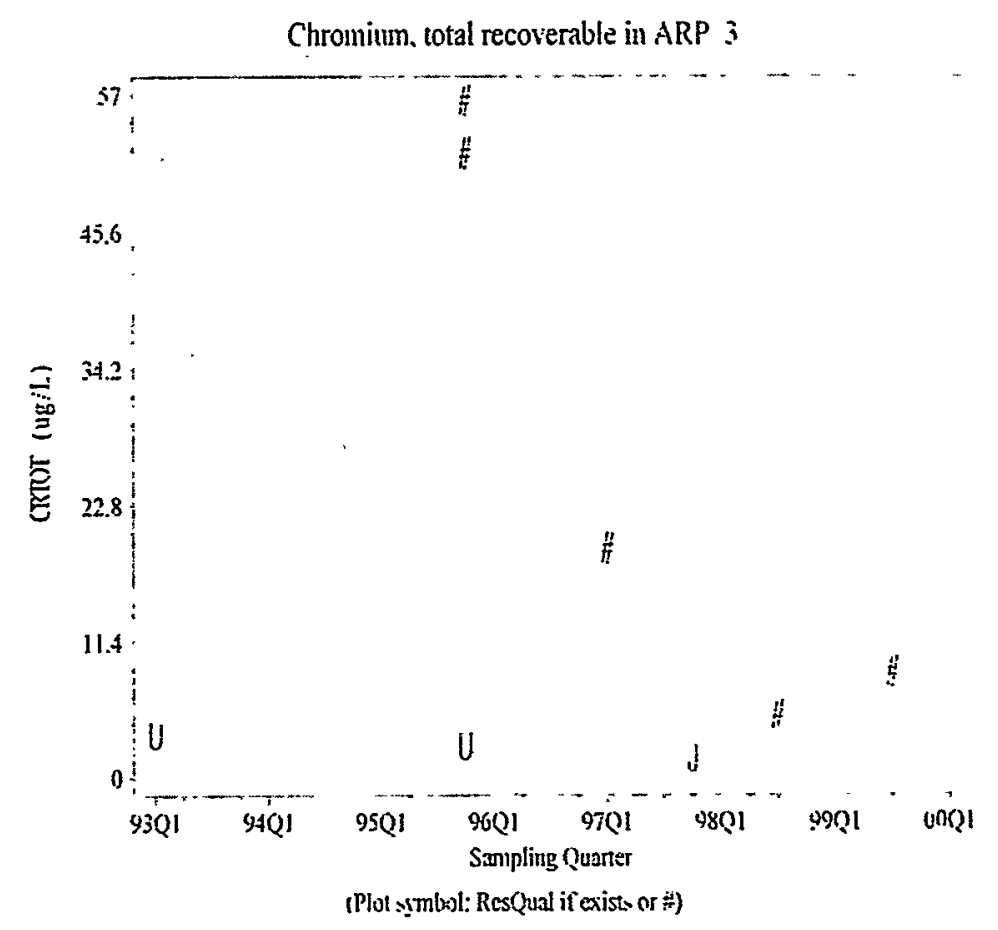


Iron, total recoverable in ARP 3

Data Retrieved by AWSA v1 from GIMS on 09NOV99 at 15:50

Well Analyte Sample_QRes_Qual Result Units

ARP 3 FETOT 189301

$\begin{array}{lll}\text { ARP } 3 & \text { FETOT 1995Q4 } & 84.7 \text { UGL }\end{array}$

ARP 3 FETOT $199504 \quad 7440$ UGL

ARP 3 FETOT 1995Q4 8400 UGL

ARP 3 FETOT 199701

ARP 3 FETOT 1998Q3

ARP 3 FETOT 199901

ARP 3 FETOT 1999 Q3

586 UGL

90 UGL

325 UGL

725 UGL

${ }_{8700}$

$\begin{array}{ll}7 & 5040 \\ 30 & \\ 3 & \\ 5 & \\ 5 & 3360\end{array}$.

1680

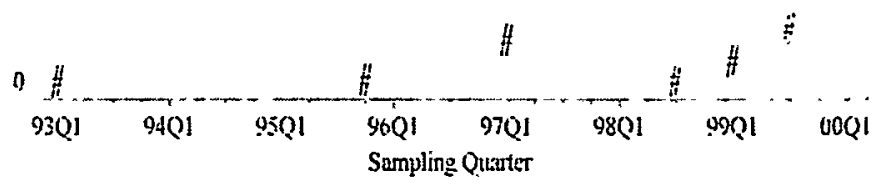

(Plot symbol: ResQual if crists ur 
Manganese, total recoverable in ARP 3

Data Retrieved by AWSA v1 from GIMS on 09NOV99 at 15:50

Well Analyte Sample_Q Res_Qual Result Units

ARP $3 \quad$ MNTOT 199301

ARP 3 MNTOT $1995 Q 4$

ARP 3 MNTOT 1995Q4

ARP 3 MNTOT 1996Q1

ARP 3 MNTOT 199701

ARP 3 MNTOT 1997Q4 J

ARP 3 MNTOT $1998 \mathrm{Q} 3 \mathrm{~J}$

ARP 3 MNTOT 1999Q1 J

ARP 3 MNTOT $199903 \mathrm{~J}$
7.36 UGL 5.32 UGL 40.5 UGL

24.3 UGL

8.4 UGL

6.59 UGL

6.44 UGL

7 UGL

9.76 UGL
Mangauese, total recorerable in ARP 3

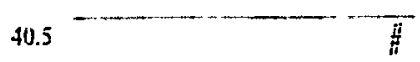

32.4

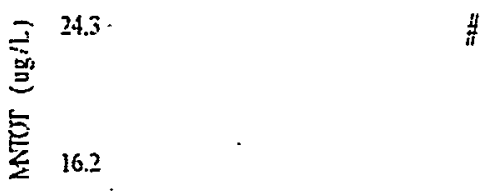

8.1

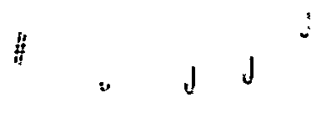

0 .

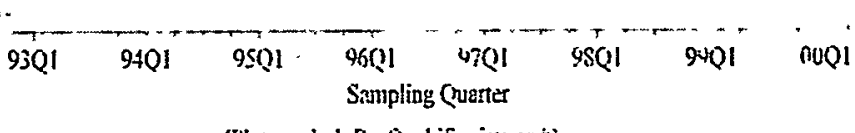


Aluminum, total recoverable in ARP 3

Data Retrieved by AWSA v1 from GIMS on 09NOV99 at 15:50

Well Analyte Sample_QRes_Qual Result Units

ARP 3 ALTOT 1993Q1

ARP 3 ALTOT $1993 Q 3$ UJ

ARP 3 ALTOT $1895 Q 4$

ARP 3 ALTOT 1995Q4

ARP 3 ALTOT 1995Q4

ARP 3 ALTOT 1996Q1

ARP 3 ALTOT 1996Q1 J

ARP 3 ALTOT 1997Q1

ARP 3 ALTOT 199704

ARP 3 ALTOT $1998 Q 3$

ARP 3 ALTOT 1999Q1 J

ARP 3 ALTOT $1999 Q 3$
39.1 UGL 20 UGL 31.2 UGL 1540 UGL 2950 UGL 1380 UGL 1530 UGL 106 UGL 70.8 UGL 36 UGL 95.2 UGL 150 UGL
Aluminum, total recoverable in ARP 3

it

it

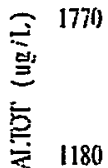

590

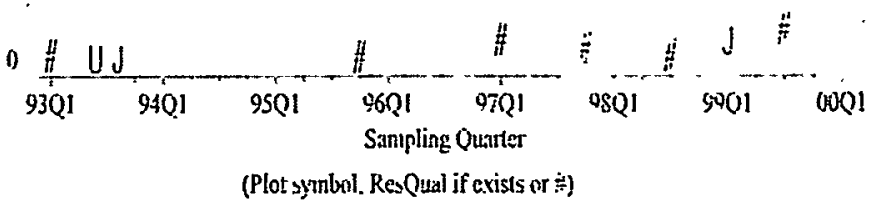


Lead, total recoverable in ARP 3

Data Retrieved by AWSA v1 from GIMS on 09NOV99 at 15:50

\begin{tabular}{|c|c|c|c|c|c|}
\hline Well & Analyte & Sample_C & Res_Qual & Result & Units \\
\hline ARP 3 & PBTOT & 1993Q1 & & & 4.49 UC \\
\hline ARP 3 & РBTOT & $1995 Q 4$ & $J$ & & 1.18 UGL \\
\hline ARP 3 & РВTOT & $1985 Q 4$ & $J$ & & 2.45 UGL \\
\hline ARP 3 & PBTOT & 1996Q1 & $J$ & & 1.94 UGL \\
\hline ARP 3 & PBTOT & 1996Q1 & $J$ & & 2.17 UGL \\
\hline ARP 3 & РВTOT & 1996Q1 & U & & 100 UGL \\
\hline ARP 3 & РВTOT & 1997Q1 & $U$ & & 5 UGL \\
\hline ARP 3 & РВTOT & $1997 Q 4$ & $U$ & & 5 UGL \\
\hline ARP 3 & РВTOT & 199803 & $\mathbf{u}$ & & 2 UGL \\
\hline ARP 3 & РВTOT & 1999Q1 & $u$ & & 47 UGL \\
\hline RP 3 & РBTOT & 1999Q3 & $u$ & & 5 UGL \\
\hline
\end{tabular}

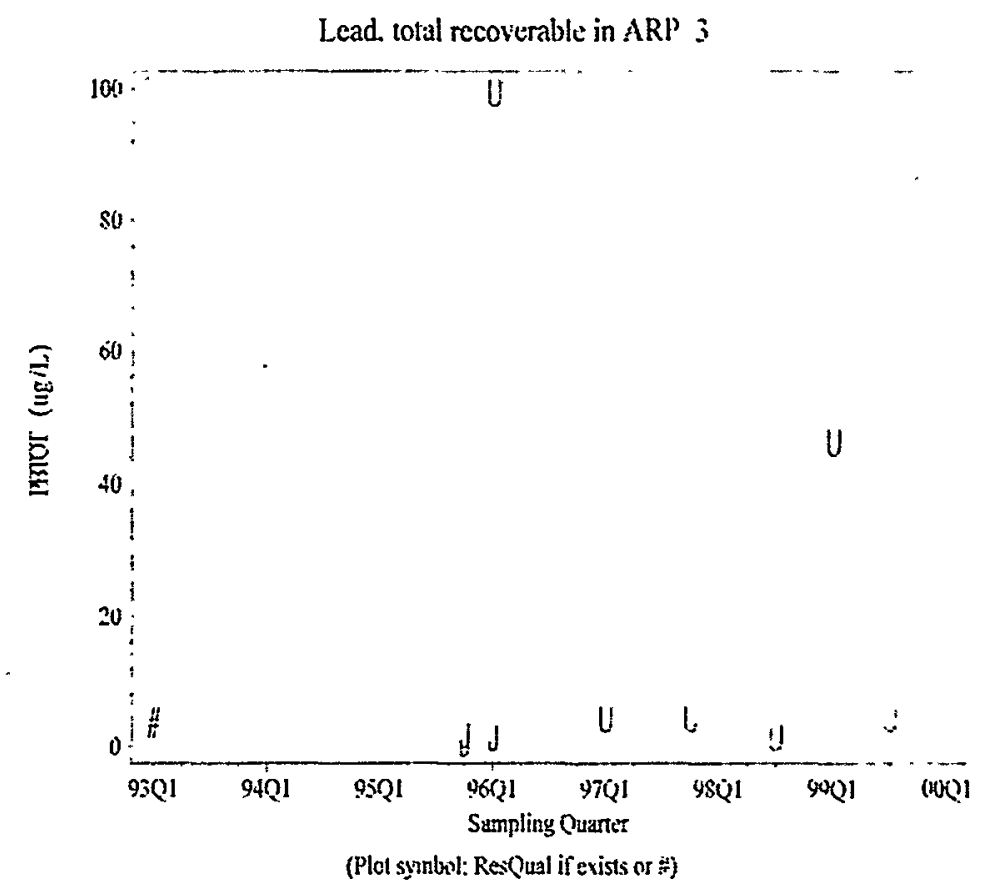


Chloride in ARP 3

Data Retrieved by AWSA vi from GIMS on 09NOV99 at 15:50

Well Analyte Sample_Q Res_Qual Result Units

$\begin{array}{llll}A R P & \mathrm{CL} & 1984 \mathrm{Q} 3000 \mathrm{UG}\end{array}$

$\begin{array}{llll}A R P & \mathrm{CL} & 1984 Q 4 & 3100 \mathrm{UGL}\end{array}$

$\begin{array}{llll}A R P & \mathrm{Cl} & 198404 & 3500 \mathrm{UGL}\end{array}$

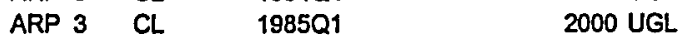

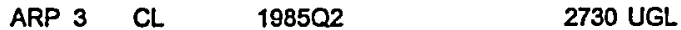

$\begin{array}{llll}\text { ARP } 3 & C L & 1986 Q 1 & 6900 \text { UGL }\end{array}$

$\begin{array}{llll}A R P & \mathrm{CL} & 1986 \mathrm{Q} & 6900 \mathrm{UGL}\end{array}$

ARP $3 \quad C L \quad 198701 \quad 3100$ UGL

ARP 3 CL 1989Q1 2900 UGL

$\begin{array}{llll}\text { ARP } 3 & \mathrm{CL} & 1989 Q 2 & 7000 \mathrm{UGL}\end{array}$

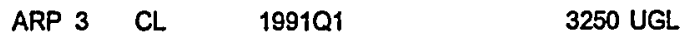

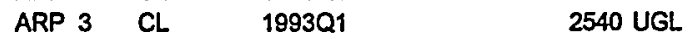

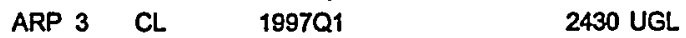

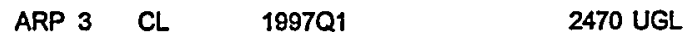

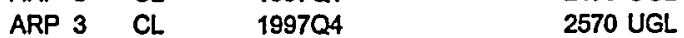

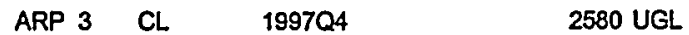

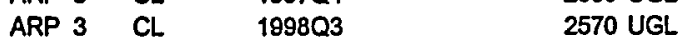

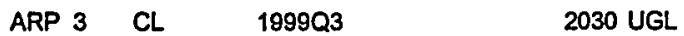

$\begin{array}{llll}A R P & C L & 1999 Q 3 & 2110 \mathrm{UGL}\end{array}$

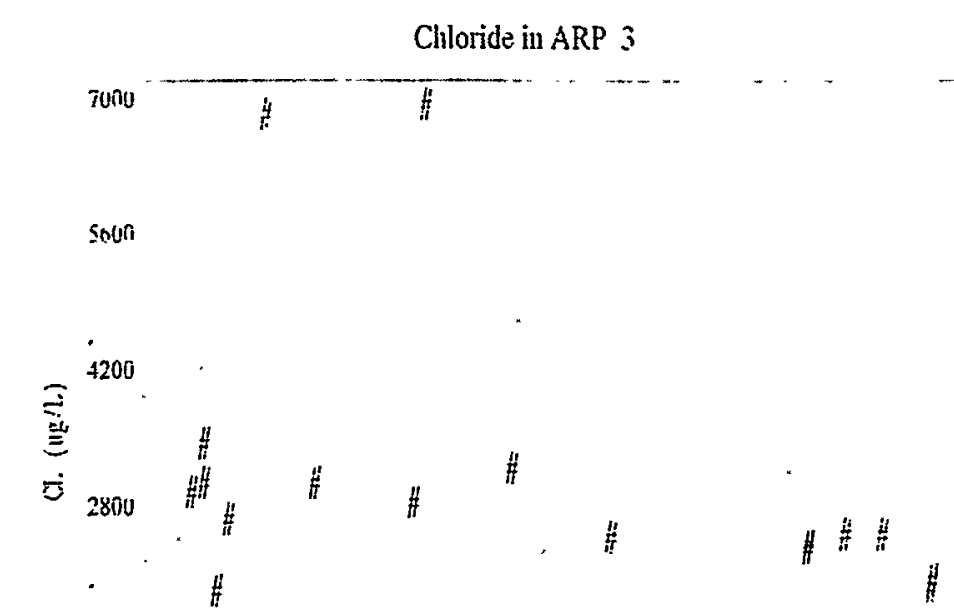

1400

0

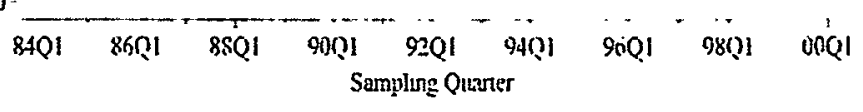

(Plot s)mbol: Res(ual if exists or $\ddot{\text { in) }}$ 
Carbon tetrachloride in ARP 3

Data Retrieved by AWSA v1 from GIMS on 09NOV99 at 15:50

\begin{tabular}{|c|c|c|c|c|}
\hline Well & Analyte & Sample_ & 2 Res_Qual Result & Units \\
\hline ARP 3 & CCL4 & $1985 Q 4$ & & 5 UGL \\
\hline ARP 3 & CCL4 & 1986Q3 & & $1 \mathrm{UGL}$ \\
\hline ARP 3 & CCL4 & $1986 Q 4$ & & $1 \mathrm{UGL}$ \\
\hline ARP 3 & CCL4 & 1987Q1 & & $1 \mathrm{UGL}$ \\
\hline ARP 3 & CCL4 & 1987Q2 & & 1 UGL \\
\hline ARP 3 & $\mathrm{CCL} 4$ & 1987Q3 & & 1 UGL \\
\hline ARP 3 & CCL4 & $1987 Q 4$ & & 25 UGL \\
\hline ARP & CCL4 & $1988 Q 1$ & & 1 UGL \\
\hline ARP & CCL4 & 1988Q1 & & $1 \mathrm{UGL}$ \\
\hline ARP 3 & $\mathrm{CCL} 4$ & 1988Q3 & & $1 \mathrm{UGL}$ \\
\hline ARP 3 & $\mathrm{CCL} 4$ & 1989Q1 & $\mathbf{U}$ & $1 \mathrm{UGL}$ \\
\hline ARP 3 & CCL4 & 1989Q3 & & 1 UGL \\
\hline ARP & CCL4 & $1989 Q 3$ & U & 10 UGL \\
\hline ARP 3 & CCL4 & 1990Q1 & & 1 UGL \\
\hline ARP 3 & CCL4 & $1990 \mathrm{Q} 3$ & & 2 UGL \\
\hline ARP 3 & CCL4 & 1991Q1 & & 2 UGL \\
\hline ARP 3 & CCL4 & 1992Q3 & UJ & 5 UGL \\
\hline ARP 3 & CCL4 & $1992 Q 4$ & $\mathbf{U}$ & 5 UGL \\
\hline ARP 3 & $\mathrm{CCL} 4$ & 199301 & $\mathbf{U}$ & 5 UGL \\
\hline ARP 3 & CCL4 & 1993Q2 & $\mathbf{U}$ & 5 UGL \\
\hline ARP 3 & $\mathrm{CCL} 4$ & $1993 Q 3$ & $U$ & 5 UGL \\
\hline ARP 3 & CCL4 & 199504 & $U$ & $0.5 \mathrm{UGL}$ \\
\hline ARP & CCL4 & $1995 Q 44$ & UJ & 0.5 UGL \\
\hline ARP & CCL4 & 1996Q1 & $\mathrm{U}$ & 2 UGL \\
\hline ARP & CCL4 & 1997Q1 & $\mathrm{U}$ & $1 \mathrm{UGL}$ \\
\hline ARP & CCL4 & 1997Q4 & $\mathbf{U}$ & $1 \mathrm{UGL}$ \\
\hline ARP & CCL4 & 1998Q3 & U. & $1 \mathrm{UGL}$ \\
\hline ARP & CCL4 & 1999Q1 & JU & 5 UGL \\
\hline ARP & CCL4 & $1999 Q 3$ & U & 1 UGL \\
\hline
\end{tabular}

Carbon tetraclloride in ARP 3

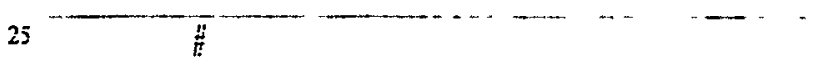

20

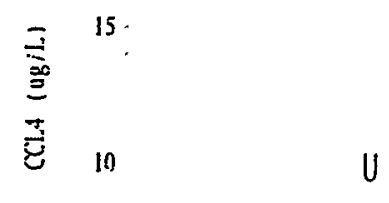

$5 \quad$ H UlW $\quad$ UU

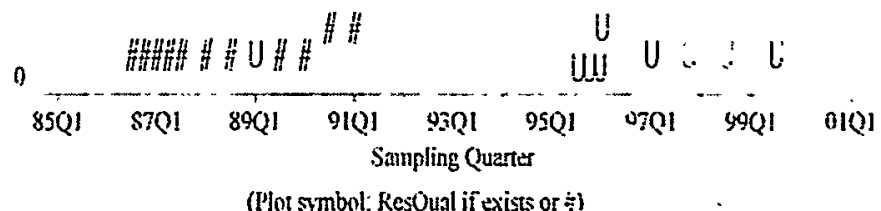


Tetrachloroethylene in ARP 3

Data Retrieved by AWSA v1 from GIMS on 09NOV99 at 15:50

\begin{tabular}{|c|c|c|c|c|}
\hline Well & Analyte & Sample_C & Res_Qual Result & Units \\
\hline ARP 3 & TCLEE & $1985 Q 4$ & & 5 UGL \\
\hline ARP 3 & TCLEE & 1986Q3 & & 6.01 UGL \\
\hline ARP 3 & TCLEE & $1986 Q 4$ & & 6.31 UGL \\
\hline ARP 3 & TCLEE & 1987Q1 & & 6.87 UGL \\
\hline ARP 3 & TCLEE & 1987Q2 & & 8.8 UGL \\
\hline ARP 3 & TCLEE & 1987Q3 & & 11.2 UGL \\
\hline ARP 3 & TCLEE & $1987 Q 4$ & & 25 UGL \\
\hline ARP 3 & TCLEE & 1988Q1 & & 12.5 UGL \\
\hline ARP 3 & TCLEE & 1988Q1 & & 12.5 UGL \\
\hline ARP 3 & TCLEE & $1988 Q 3$ & & 15.8 UGL \\
\hline ARP 3 & TCLEE & $1988 Q 3$ & & 29.8 UGL \\
\hline ARP 3 & TCLEE & 1989Q1 & & 28.5 UGL \\
\hline ARP 3 & TCLEE & 1989Q1 & & 29 UGL \\
\hline ARP 3 & TCLEE & $1989 Q 2$ & & 2.93 UGL \\
\hline ARP 3 & TCLEE & 1989Q3 & & 26 UGL \\
\hline ARP 3 & TCLEE & 1989Q3 & & 45 UGL \\
\hline ARP 3 & TCLEE & $1989 Q 4$ & & 49 UGL \\
\hline ARP 3 & TCLEE & 1990Q1 & & 47 UGL \\
\hline ARP 3 & TCLEE & 1990Q2 & & 56 UGL \\
\hline ARP 3 & TCLEE & $1990 Q 3$ & & 28 UGL \\
\hline ARP 3 & TCLEE & 1990Q4 & & 34.3 UGL \\
\hline ARP 3 & TCLEE & 1991Q1 & & 34.8 UGL \\
\hline ARP 3 & TCLEE & 1991Q1 & & 44 UGL \\
\hline ARP 3 & TCLEE & 1991Q4 & U & 10 UGL \\
\hline ARP 3 & TCLEE & 199201 & U & 50 UGL \\
\hline ARP 3 & TCLEE & $1992 Q 2$ & & 11.8.UGL \\
\hline ARP 3 & TCLEE & $1992 Q 3$ & $\mathrm{~J}$ & 13 UGL \\
\hline ARP 3 & TCLEE & $1992 Q 4$ & & 20.2 UGL \\
\hline ARP 3 & TCLEE & 1993Q1 & & 18.5 UGL \\
\hline ARP 3 & TCLEE & 199302 & & 16.5 UGL \\
\hline ARP 3 & TCLEE & 1993Q3 & & 12 UGL \\
\hline ARP 3 & TCLEE & 1995Q4 & & 9.4 UGL \\
\hline ARP 3 & TCLEE & 1995Q4 & $\mathbf{J}$ & 9.1 UGL \\
\hline ARP 3 & TCLEE & 1996Q1 & & 10.9 UGL \\
\hline ARP 3 & TCLEE & 1997Q1 & & 14 UGL \\
\hline ARP 3 & TCLEE & $1997 Q 4$ & $\mathbf{U}$ & 1 UGL \\
\hline ARP 3 & TCLEE & 1998Q3 & & 18.1 UGL \\
\hline ARP 3 & TCLEE & $1999 Q 1$ & $\mathbf{U}$ & 25 UGL \\
\hline ARP 3 & TCLEE & $1999 Q 3$ & & 9.85 UGL \\
\hline
\end{tabular}

Tetrachloroetlyylene in ARP 3

so

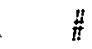

44. $\$$<smiles>C=CC=C</smiles>

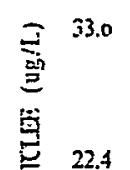

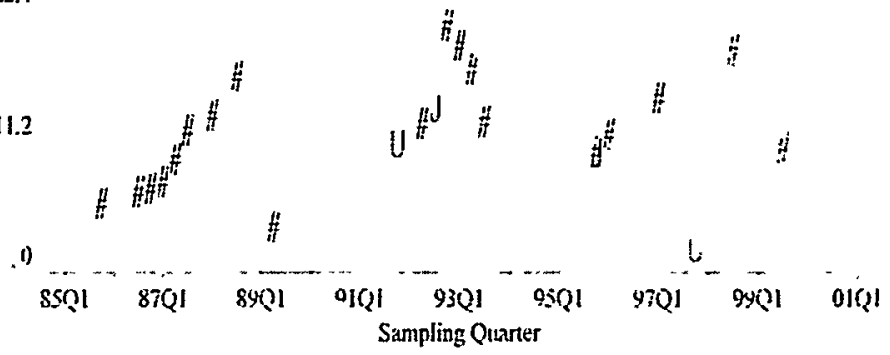

(Phot symbol: kesfrual if exists or ô 
Trichloroethylene in ARP 3

Data Retrieved by AWSA v1 from GIMS on 09NOV99 at 15:50

\begin{tabular}{|c|c|c|}
\hline Well & Analyte & Sample_ \\
\hline ARP 3 & TRCLE & $1985 Q 4$ \\
\hline ARP 3 & TRCLE & 188602 \\
\hline ARP 3 & TRCLE & 1986Q3 \\
\hline ARP 3 & TRCLE & $1986 Q 4$ \\
\hline ARP 3 & TRCLE & 1987Q1 \\
\hline ARP 3 & TRCLE & 1987Q2 \\
\hline ARP 3 & TRCLE & 1987Q3 \\
\hline ARP 3 & TRCLEE & $1987 Q 4$ \\
\hline ARP 3 & TRCLE & $1988 Q 1$ \\
\hline ARP 3 & TRCLE & 198801 \\
\hline ARP 3 & TRCLE & $1988 Q 3$ \\
\hline ARP 3 & TRCLE & 1888Q3 \\
\hline ARP 3 & TRCLE & $1889 Q 1$ \\
\hline ARP 3 & TRCLE & 1989Q1 \\
\hline ARP 3 & TRCLE & $1989 Q 2$ \\
\hline ARP 3 & TRCLE & $1989 Q 3$ \\
\hline ARP 3 & TRCLE & $1989 Q 3$ \\
\hline ARP 3 & TRCLE & 198904 \\
\hline ARP 3 & TRCLE & $1990 Q 1$ \\
\hline ARP 3 & TRCLE & $1990 Q 2$ \\
\hline ARP 3 & TRCLE & 1990Q3 \\
\hline ARP 3 & TRCLE & $1990 Q 4$ \\
\hline ARP 3 & TRCLE & 1991Q1 \\
\hline ARP 3 & TRCLE & 1991Q1 \\
\hline ARP 3 & TRCLE & 1991Q4 \\
\hline ARP 3 & TRCLE & 1992Q1 \\
\hline ARP 3 & TRCLE & 199202 \\
\hline ARP 3 & TRCLE & $1992 Q 3$ \\
\hline ARP 3 & TRCLE & 1992Q4 \\
\hline ARP 3 & TRCLE & 1993Q1 \\
\hline ARP 3 & TRCLE & $1993 Q 2$ \\
\hline ARP 3 & TRCLE & 1993Q3 \\
\hline ARP 3 & TRCLE & $1995 Q 4$ \\
\hline ARP 3 & TRCLE & $1995 Q 4$ \\
\hline ARP 3 & TRCLE & 1996Q1 \\
\hline ARP 3 & TRCLE & 1997Q1 \\
\hline ARP 3 & TRCLE & $1997 Q 4$ \\
\hline ARP 3 & TRCLE & 1998Q3 \\
\hline ARP 3 & TRCLE & 1999Q1 \\
\hline ARP 3 & TRCLE & $1999 Q 3$ \\
\hline
\end{tabular}
29.5 UGL 66.6 UGL

1 UGL

148 UGL

248 UGL

76.2 UGL

176 UGL

12.5 UGL

12.5 UGL

213 UGL

214 UGL

337 UGL

490 UGL

549 UGL

451 UGL

840 UGL

856 UGL

608 UGL

757 UGL

1209 UGL

655 UGL

520 UGL

557 UGL

133 UGL

76.1 UGL

161 UGL

126 UGL

263 UGL

199 UGL

200 UGL

148 UGL

111 UGL

108 UGL

101 UGL

130 UGL

1.02 UGL

186 UGL

74 UGL

77.6 UGL
Trichloroethylene in ARP 3
1205

$$
4
$$

9672

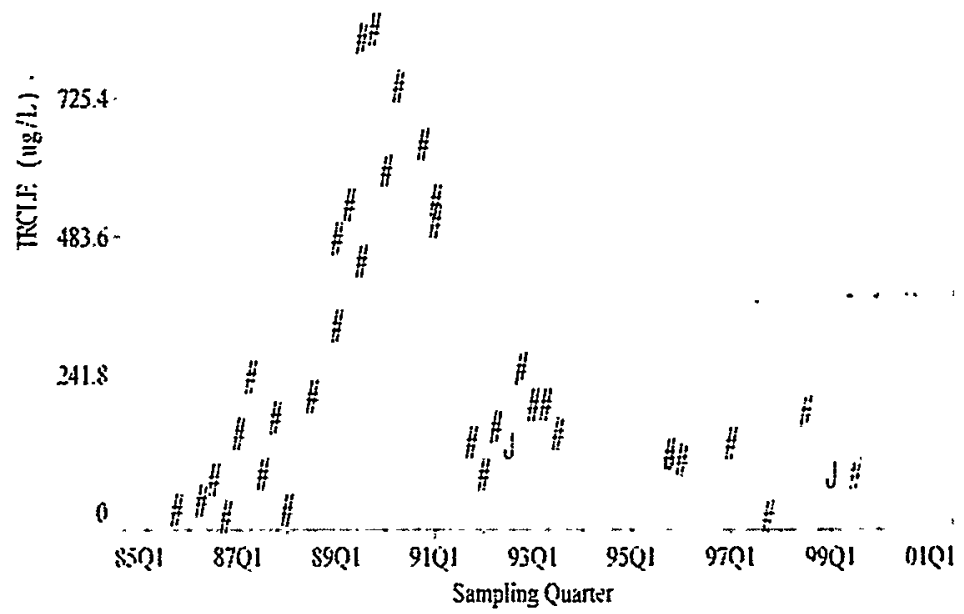

(Plor syntool: ResQunl if uxists or ä) 
Gross alpha in ARP 3

Data Retrieved by AWSA v1 from GIMS on 09NOV99 at 15:50

\begin{tabular}{|c|c|c|}
\hline Well & Analyte & Sample \\
\hline ARP 3 & ALPHAG & 198403 \\
\hline ARP 3 & ALPHAG & 198404 \\
\hline ARP 3 & ALPHAG & 1985Q1 \\
\hline ARP 3 & ALPHAG & 1985Q2 \\
\hline ARP 3 & ALPHAG & 1986Q1 \\
\hline ARP 3 & ALPHAG & 1987Q1 \\
\hline ARP 3 & ALPHAG & 1988Q1 \\
\hline ARP 3 & ALPHAG & 1989Q1 \\
\hline ARP 3 & ALPHAG & 1989Q2 \\
\hline ARP 3 & ALPHAG & $1991 Q 1$ \\
\hline ARP 3 & ALPHAG & 1993Q1 \\
\hline ARP 3 & ALPHAG & 199504 \\
\hline ARP 3 & ALPHAG & 1995Q4 \\
\hline ARP 3 & ALPHAG & 1995Q4 \\
\hline ARP 3 & ALPHAG & 1997Q1 \\
\hline ARP 3 & ALPHAG & 199704 \\
\hline ARP 3 & ALPHAG & 1997Q4 \\
\hline ARP 3 & ALPHAG & 199803 \\
\hline ARP 3 & ALPHAG & 1999Q3 \\
\hline
\end{tabular}

Units

$10 \mathrm{PCl}$

$2 \mathrm{PCL}$

$4 \mathrm{PCL}$

$2 \mathrm{PCL}$

$2 \mathrm{PCL}$

$3 \mathrm{PCL}$

$3 \mathrm{PCL}$

3.45 PCL

$3 \mathrm{PCL}$

$2 \mathrm{PCL}$

$2 \mathrm{PCl}$

$1.58 \mathrm{PCL}$

$2.19 \mathrm{PCL}$

$2.38 \mathrm{PCL}$

$1.72 \mathrm{PCL}$

$2.99 \mathrm{PCL}$

$3.01 \mathrm{PCL}$

$3.63 \mathrm{PCL}$

$0.606 \mathrm{PCL}$
Gross alpha in ARP 3

$10-7$.

8

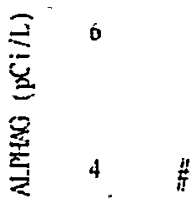

\#. .

$\stackrel{i j}{*}$

$2 \# \# \# \quad U \quad U \quad \frac{k}{\#}$

0 .

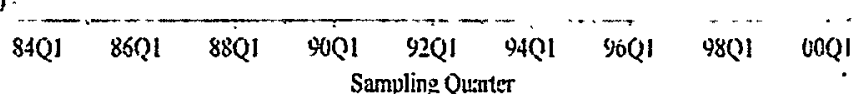
(Plot synhol: Rer(lual if exists ur $\stackrel{\text { ) }}{ }$ 
1,1,1-Trichloroethane in ARP 3

Data Retrieved by AWSA v1 from GIMS on 09NOV99 at 15:50

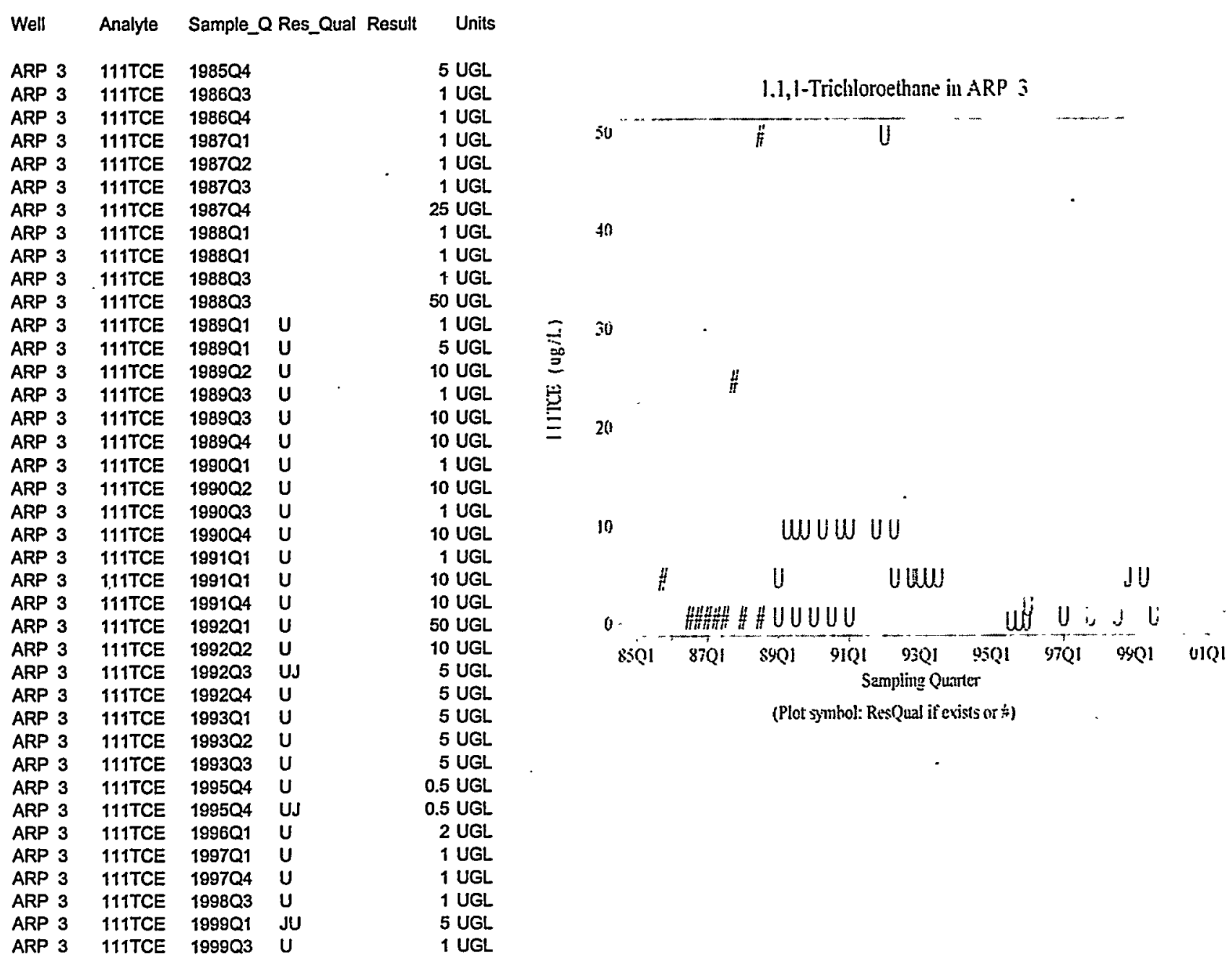


1,1-Dichloroethylene in ARP 3

Data Retrieved by AWSA v1 from GIMS on 09NOV99 at 15:50

\begin{tabular}{|c|c|c|}
\hline Well & Analyte & $m p$ \\
\hline ARP 3 & 11DCE & $1985 Q 4$ \\
\hline ARP 3 & 11DCE & $1988 Q 3$ \\
\hline ARP 3 & 11DCE & 1989Q1 \\
\hline ARP 3 & 11DCE & $1989 Q 2$ \\
\hline ARP 3 & 11DCE & $1989 Q 3$ \\
\hline ARP 3 & 11DCE & 1989Q3 \\
\hline ARP 3 & 11DCE & $1989 Q 4$ \\
\hline ARP 3 & 11DCE & 1990Q1 \\
\hline ARP 3 & 11DCE & $1990 Q 2$ \\
\hline ARP 3 & 11DCE & $1990 Q 4$ \\
\hline ARP 3 & 11DCE & 1991Q1 \\
\hline ARP 3 & 11DCE & 1991Q1 \\
\hline ARP 3 & 11DCE & 1991Q4 \\
\hline ARP 3 & 11DCE & $1992 Q 1$ \\
\hline ARP 3 & 11DCE & $1992 Q 2$ \\
\hline ARP 3 & 11DCE & 199301 \\
\hline ARP 3 & 11DCE & 199302 \\
\hline ARP 3 & 11DCE & 1993Q3 \\
\hline ARP 3 & 11DCE & 1995Q4 \\
\hline ARP 3 & 11DCE & $1995 Q 4$ \\
\hline ARP 3 & 11DCE & $1997 Q 4$ \\
\hline & 11DCE & $1998 Q 3$ \\
\hline & 11DCE & 1999Q3 \\
\hline
\end{tabular}

Units

5 UGL 27.4 UGL 5 UGL 10 UGL 1 UGL 10 UGL 10 UGL 1 UGL 10 UGL 10 UGL 1 UGL 10 UGL 10 UGL 50 UGL 10 UGL 5 UGL 5 UGL 5 UGL 0.5 UGL 0.5 UGL 1 UGL 1 UGL 1 UGL
1.1-Dichloroethylene in ARP 3

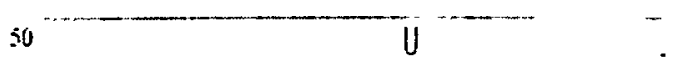

4)

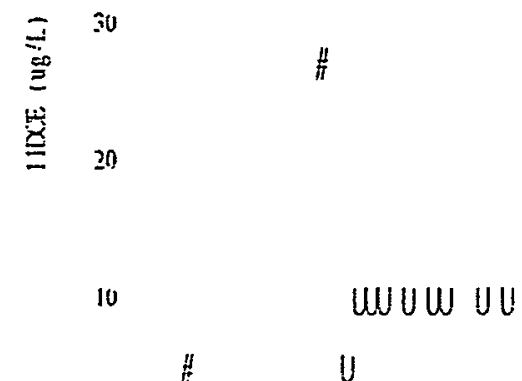

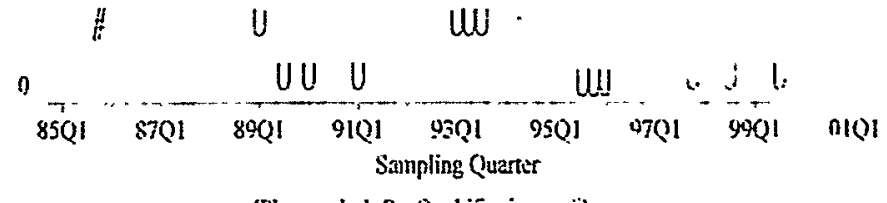


1,1-Dichloroethane in ARP 3

Data Retrieved by AWSA v1 from GIMS on 09NOV99 at 15:50

\begin{tabular}{llllr} 
Well & Analyte & \multicolumn{2}{c}{ Sample_Q Res_Qual Result } & Units \\
& & & & \\
ARP 3 & $11 D C L E$ & $1985 Q 4$ & & 5 UGL \\
ARP 3 & $11 D C L E$ & $1989 Q 3$ & $U$ & 1 UGL \\
ARP 3 & $11 D C L E$ & $1989 Q 3$ & $U$ & 10 UGL \\
ARP 3 & $11 D C L E$ & $1990 Q 1$ & $U$ & 1 UGL \\
ARP 3 & $11 D C L E$ & $1991 Q 1$ & $U$ & 1 UGL \\
ARP 3 & $11 D C L E$ & $1993 Q 1$ & $U$ & 5 UGL \\
ARP 3 & $11 D C L E$ & $1993 Q 2$ & $U$ & 5 UGL \\
ARP 3 & $11 D C L E$ & $1993 Q 3$ & $U$ & 5 UGL \\
ARP 3 & $11 D C L E$ & $1995 Q 4$ & $U$ & 0.5 UGL \\
ARP 3 & $11 D C L E$ & $1995 Q 4$ & $U$ J & 0.5 UGL \\
ARP 3 & $11 D C L E$ & $1997 Q 4$ & $U$ & 1 UGL \\
ARP 3 & $11 D C L E$ & $1998 Q 3$ & $U$ & 1 UGL \\
ARP 3 & $11 D C L E$ & $1999 Q 3$ & $U$ & 1 UGL
\end{tabular}

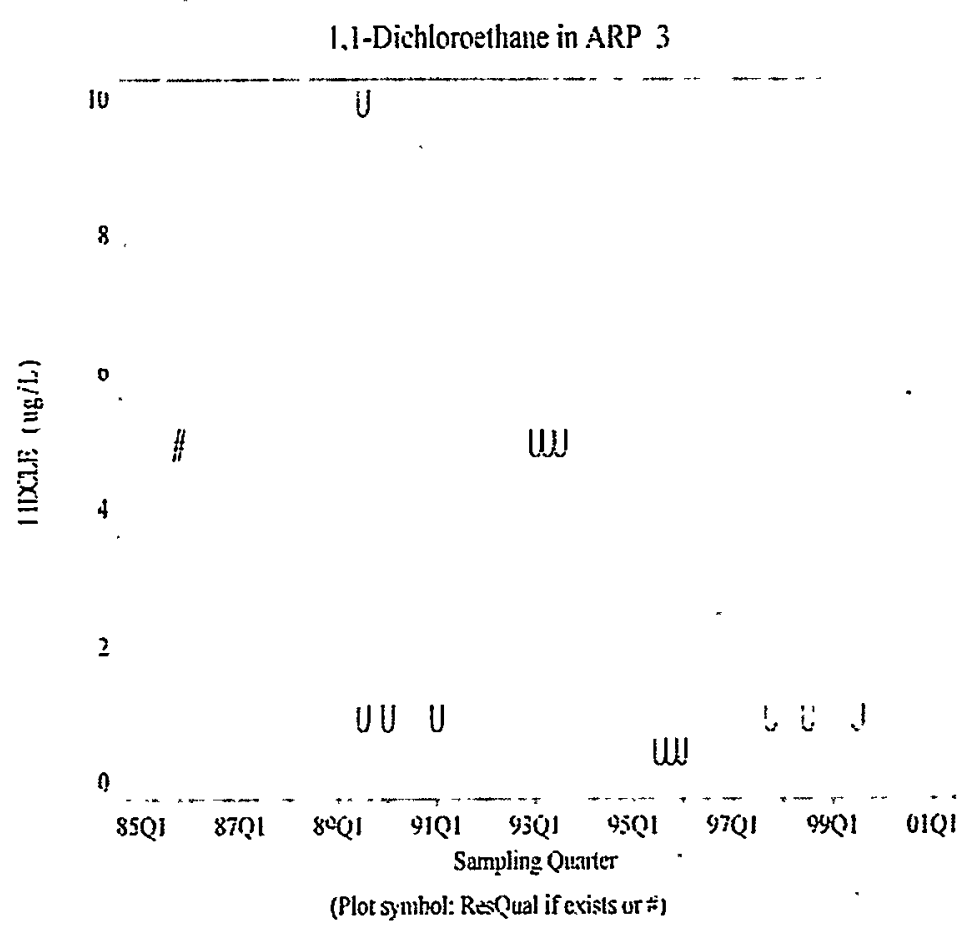


Barium, total recoverable in ARP 3

Data Retrieved by AWSA v1 from GIMS on 09NOV99 at 15:50

$\begin{array}{lllr}\text { Well } & \text { Analyte } & \text { Sample_Q Res_Qual Result } & \text { Units } \\ & & & \\ \text { ARP 3 } & \text { BATOT } & 1993 Q 1 & 5.21 \text { UGL } \\ \text { ARP 3 } & \text { BATOT } & 1995 Q 4 & 6.08 \text { UGL } \\ \text { ARP 3 } & \text { BATOT } & 1995 Q 4 & 42 \text { UGL } \\ \text { ARP 3 } & \text { BATOT } & 1996 Q 1 & 47.1 \text { UGL } \\ \text { ARP 3 } & \text { BATOT } & 1997 Q 4 & 5.69 \text { UGL } \\ \text { ARP 3 } & \text { BATOT } & 1998 Q 3 & 6.78 \text { UGL } \\ \text { ARP 3 } & \text { BATOT } & 1999 Q 3 & 7.91 \text { UGL }\end{array}$

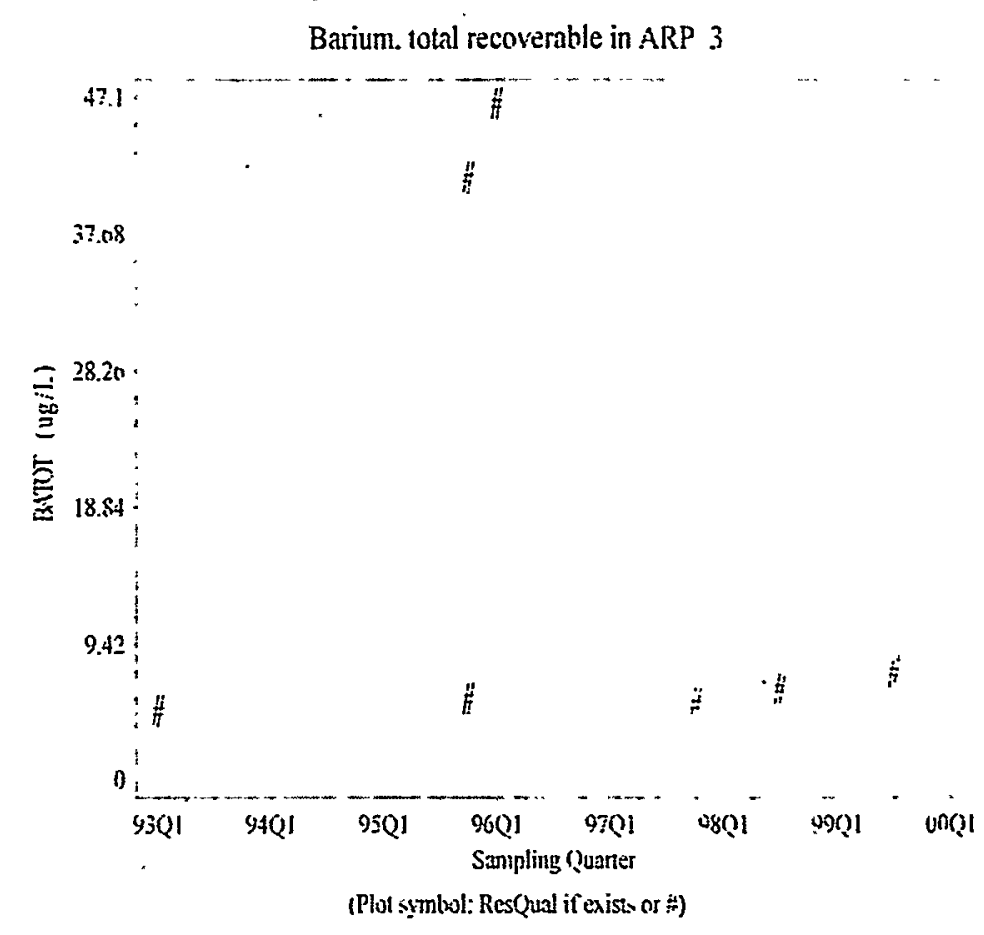


Nonvolatile beta in ARP 3

Data Retrieved by AWSA v1 from GIMS on 09NOV99 at 15:50

\begin{tabular}{|c|c|c|c|c|}
\hline Well & Analyte & Sample_ & Res_Qual & Result \\
\hline ARP 3 & BETAG & $1984 Q 3$ & & $7 \mathrm{PCL}$ \\
\hline ARP 3 & BETAG & $1984 Q 4$ & & $4 \mathrm{PCL}$ \\
\hline ARP 3 & BETAG & 1985Q1 & & $5 \mathrm{PC}$ \\
\hline ARP 3 & BETAG & $1985 \mathrm{Q} 2$ & & $3 P C$ \\
\hline ARP 3 & BETAG & 198601 & & $3 \mathrm{PC}$ \\
\hline ARP 3 & BETAG & 1987Q1 & & $2 \mathrm{PC}$ \\
\hline ARP 3 & BETAG & 1988Q1 & & $2.7 \mathrm{PC}$ \\
\hline ARP 3 & BETAG & 1989Q2 & $U$ & $2 \mathrm{PCL}$ \\
\hline ARP 3 & BETAG & 1991Q1 & U & $2 \mathrm{PCL}$ \\
\hline ARP 3 & BETAG & 1993Q1 & U & $2 \mathrm{PCL}$ \\
\hline ARP 3 & BETAG & $1995 Q 4$ & & $2.05 \mathrm{~F}$ \\
\hline ARP 3 & BETAG & $1995 Q 4$ & & $4.43 \mathrm{PCl}$ \\
\hline RP 3 & BETAG & $1995 Q 4$ & UI & $0.779 \mathrm{PCL}$ \\
\hline ARP 3 & BETAG & $1997 Q 4$ & $J$ & $3.02 \mathrm{PCL}$ \\
\hline ARP 3 & BETAG & 1997Q4 & $J$ & 3.61 PCL \\
\hline ARP 3 & BETAG & $1998 Q 3$ & & $3.03 P C$ \\
\hline ARP 3 & BETAG & 1999Q3 & $J$ & 1.78 \\
\hline
\end{tabular}

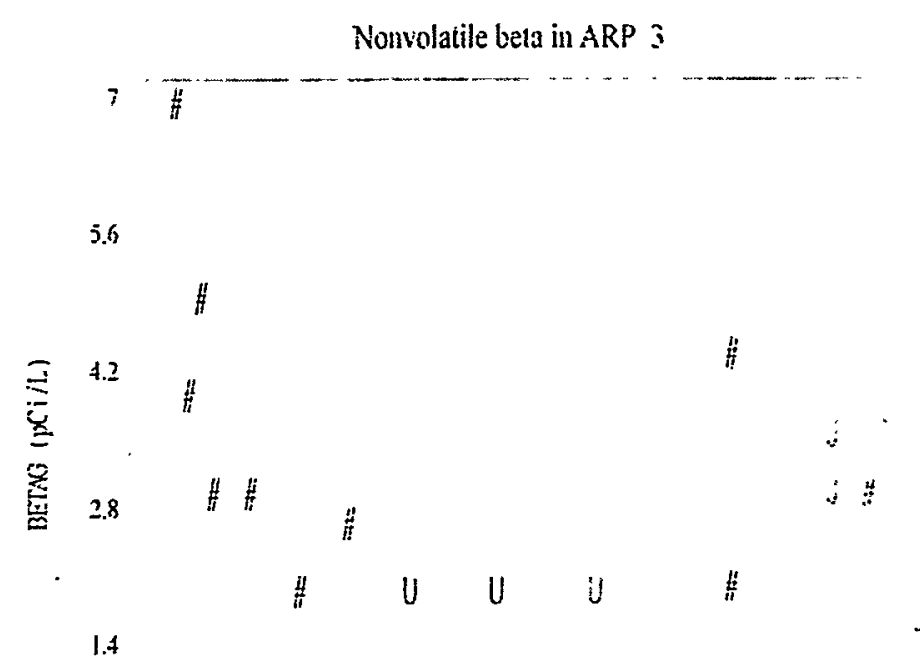

U1

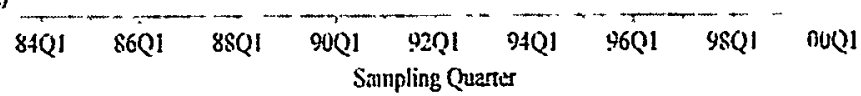
(Plo: symbol: Resuual if exists or 
Cobalt, total recoverable in ARP 3

Data Retrieved by AWSA v1 from GIMS on 09NOV99 at 15:50

Well Analyte Sample_Q Res_Qual Result Units

$\begin{array}{lllll}\text { ARP } 3 & \text { COTOT } 1995 Q 4 & \mathrm{~J} & 1.99 \text { UGL }\end{array}$

ARP 3 COTOT $199504 U$ COTL

ARP 3 COTOT $199704 U U$ UGL

ARP 3 COTOT 1998 Q 3 C.379 UGL

ARP 3 COTOT $199903 \mathrm{U}$ (
Cobalt, total recoverable in ARP 3

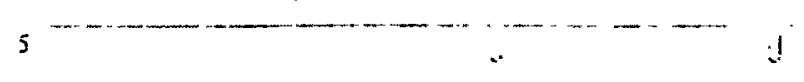

4

U

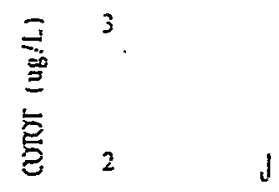

1

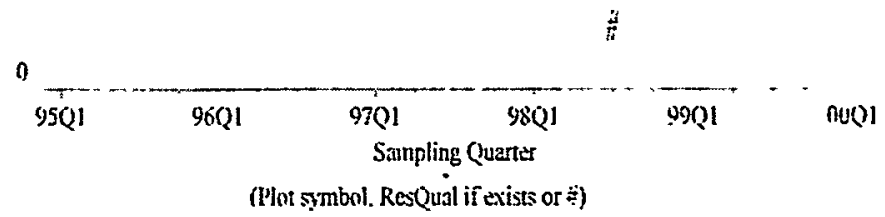


Copper, total recoverable in ARP 3

Data Retrieved by AWSA v1 from GIMS on 09NOV99 at 15:50

$\begin{array}{lrrlr}\text { Well } & \text { Analyte } & \text { Sample_Q Res_Qual Result } & \text { Units } \\ \text { ARP 3 } & \text { CUTOT } & 1995 Q 4 & \text { U } & 2.38 \text { UGL } \\ \text { ARP 3 } & \text { CUTOT } & 1995 Q 4 & \text { U } & 4 \text { UGL } \\ \text { ARP 3 } & \text { CUTOT } & 1997 Q 4 & \text { U } & 5 \text { UGL } \\ \text { ARP 3 } & \text { CUTOT } & 1998 Q 3 & & 1.3 \text { UGL } \\ \text { ARP 3 } & \text { CUTOT } & 1999 Q 3 & \text { U } & 5 \text { UGL }\end{array}$

\section{Copper. total recorerable in $A R P^{3}$}

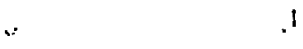

$4 \quad$ U

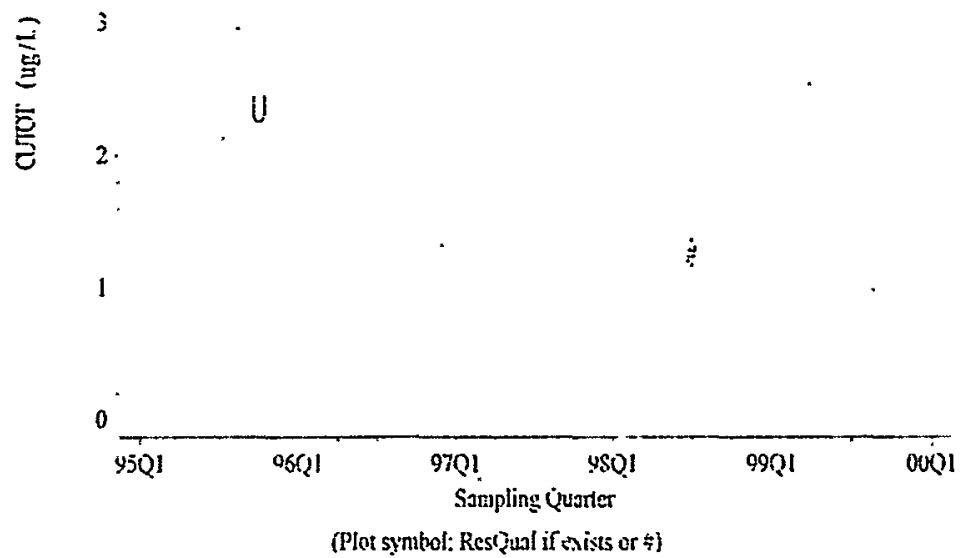

[Plot synthol: Res(zual if tnists or t) 
Cyanide in ARP 3

Data Retrieved by AWSA v1 from GIMS on 09NOV99 at 15:50

$\begin{array}{llrlr}\text { Well } & \text { Analyte } & \text { Sample_Q Res_Qual Result } & \text { Units } \\ & & & \\ \text { ARP 3 } & \text { CYN } & 1984 Q 3 & 5 \text { UGL } \\ \text { ARP 3 } & \text { CYN } & 1984 Q 4 & & 5 \text { UGL } \\ \text { ARP 3 } & \text { CYN } & 1995 Q 4 & U & 3.96 \text { UGL } \\ \text { ARP 3 } & \text { CYN } & 1995 Q 4 & U & 10 \text { UGL } \\ \text { ARP 3 } & \text { CYN } & 1997 Q 4 & \text { U } & 10 \text { UGL } \\ \text { ARP 3 } & \text { CYN } & 1998 Q 3 & \text { UJ } & 10 \text { UGL } \\ \text { ARP 3 } & \text { CYN } & 1999 Q 3 & U & 10 \text { UGL } \\ \text { ARP 3 } & \text { CYN } & 1999 Q 3 & \text { U } & 10 \text { UGL }\end{array}$

Cyanide in ARP 3

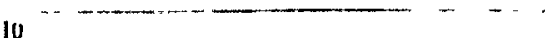

$i^{-}$

8

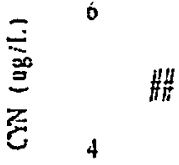

U

2

0

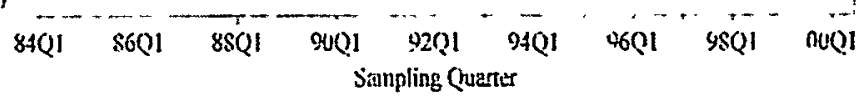
(I'lot symbal. RexQual if exists or क्)

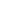


Fluoride in ARP 3

Data Retrieved by AWSA v1 from GIMS on 09NOV99 at 15:50

$\begin{array}{llll}\text { Well } & \text { Analyte } & \text { Sample_Q Res_Q } \\ & & & \\ \text { ARP 3 } & \text { F } & 1984 Q 3 & \\ \text { ARP 3 } & \text { F } & 1984 Q 4 & \\ \text { ARP 3 } & \text { F } & 1984 Q 4 & \\ \text { ARP 3 } & \text { F } & 1985 Q 1 & \\ \text { ARP 3 } & \text { F } & 1985 Q 2 & \\ \text { ARP 3 } & F & 1987 Q 1 & \\ \text { ARP 3 } & \text { F } & 1989 Q 2 & U \\ \text { ARP 3 } & \text { F } & 1991 Q 1 & U \\ \text { ARP 3 } & \text { F } & 1993 Q 1 & U \\ \text { ARP 3 } & \text { F } & 1993 Q 1 & U \\ \text { ARP 3 } & \text { F } & 1997 Q 1 & U \\ \text { ARP 3 } & \text { F } & 1997 Q 4 & U J \\ \text { ARP 3 } & \text { F } & 1997 Q 4 & U J \\ \text { ARP 3 } & \text { F } & 1998 Q 3 & U \\ \text { ARP 3 } & \text { F } & 1999 Q 3 & U \\ \text { ARP 3 } & \text { F } & 1999 Q 3 & U\end{array}$

100 UGL 100 UGL

100 UGL 100 UGL 90 UGL 100 UGL 100 UGL 100 UGL 100 UGL 100 UGL 100 UGL 50 UGL 50 UGL 50 UGL 50 UGL 50 UGL

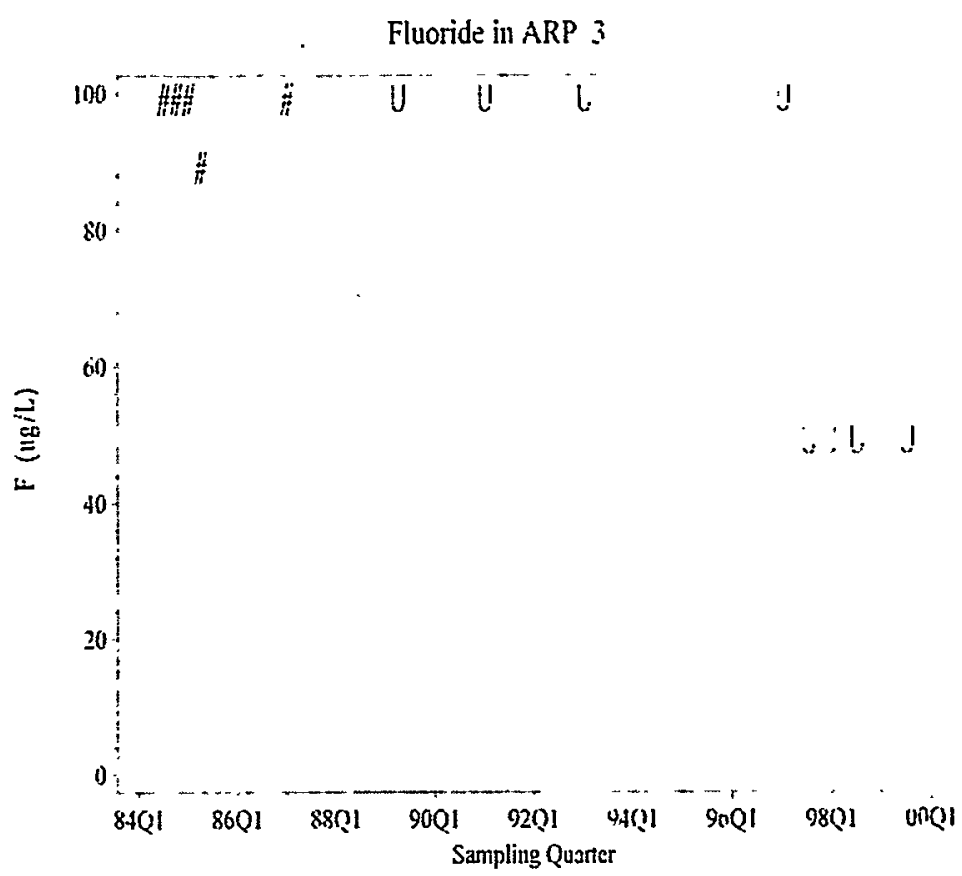

(Plol s:gmlol: ResQual it exists of t) 
Mercury, total recoverable in ARP 3

Data Retrieved by AWSA v1 from GIMS on 09NOV99 at 15:50

Well Analyte Sample_Q Res_Qual Result Units

ARP 3 HGTOT 1993Q1 U 0.2 UGL

ARP 3 HGTOT 199504 0.3 UGL

ARP 3 HGTOT 199504 U 0.2 UGL

ARP 3 HGTOT $1997 Q 4 U$ U 4.2 UGL

ARP 3 HGTOT $199803 \quad 0.661$ UGL

ARP 3 HGTOT 1999Q3 U 0.2 UGL

ARP 3 HGTOT 1999Q3 U
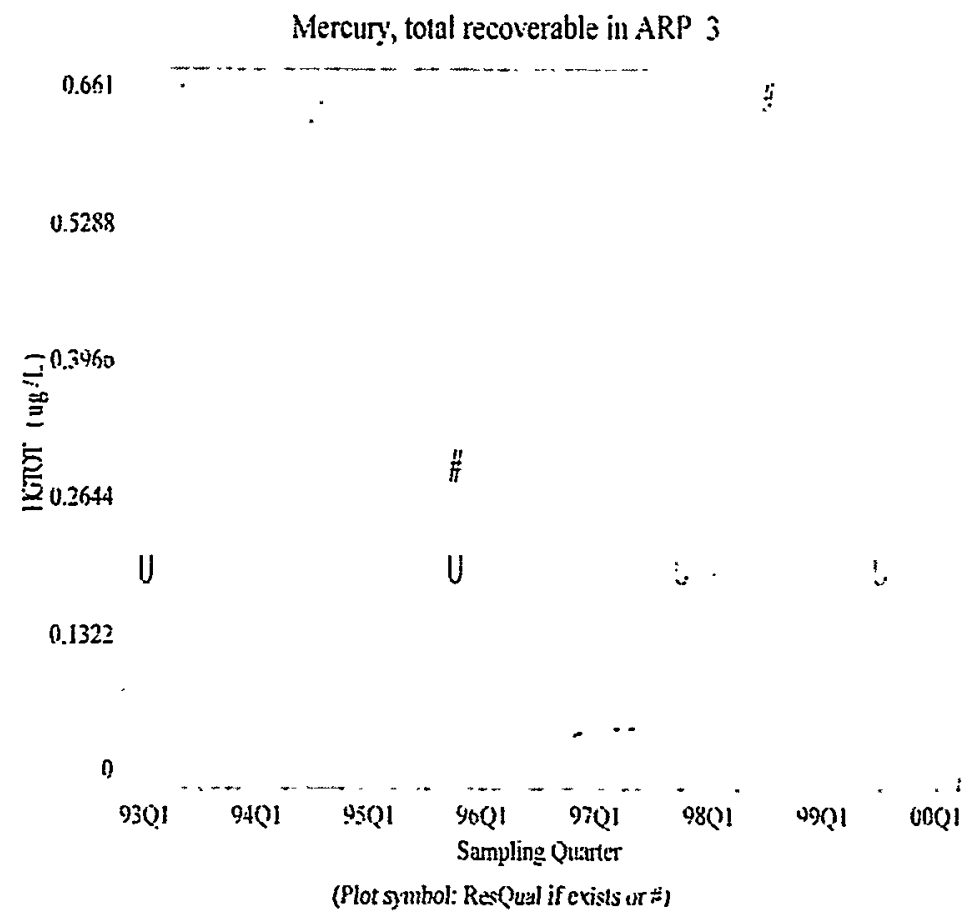
Nickel, total recoverable in ARP 3

Data Retrieved by AWSA v1 from GIMS on 09NOV99 at 15:50

\begin{tabular}{llllr} 
Well & Analyte & \multicolumn{2}{c}{ Sample_Q Res_Qual Result } & Units \\
& & & \\
ARP 3 & NITOT & 199504 & & 30.6 UGL \\
ARP 3 & NITOT & $1995 Q 4$ & U & 3.85 UGL \\
ARP 3 & NITOT & $1996 Q 1$ & & 26.5 UGL \\
ARP 3 & NITOT & $1997 Q 4$ & U & 5 UGL \\
ARP 3 & NITOT & $1998 Q 3$ & & 3.56 UGL \\
ARP 3 & NITOT & $1999 Q 3$ & 5.12 UGL
\end{tabular}

Nickel, total recorerable in ARP 3

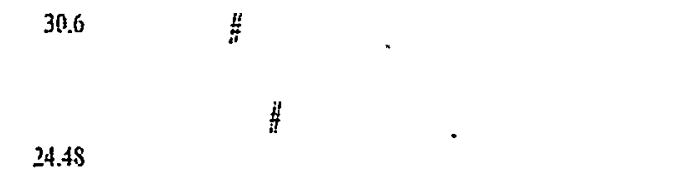

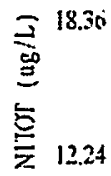

612
9501
(Plot symbol. ResQual if exists or a j)


Nitrate-nitrite as nitrogen in ARP 3

Data Retrieved by AWSA v1 from GIMS on 09NOV99 at 15:50

Well Analyte Sample_Q Res_Qual Result Units

ARP $3 \quad$ NO3NO2 $198902 \quad 390$ UGL

ARP 3 NO3NO2 1993Q1 200 UGL

ARP 3 NO3NO2 1997Q1 178 UGL

ARP $3 \quad$ NO3NO2 $1997 Q 4 \quad 190 \mathrm{UGL}$

ARP 3 NO3NO2 $199803 \quad 220$ UGL

ARP $3 \quad$ NO3NO2 1999 Q3 130 UGL
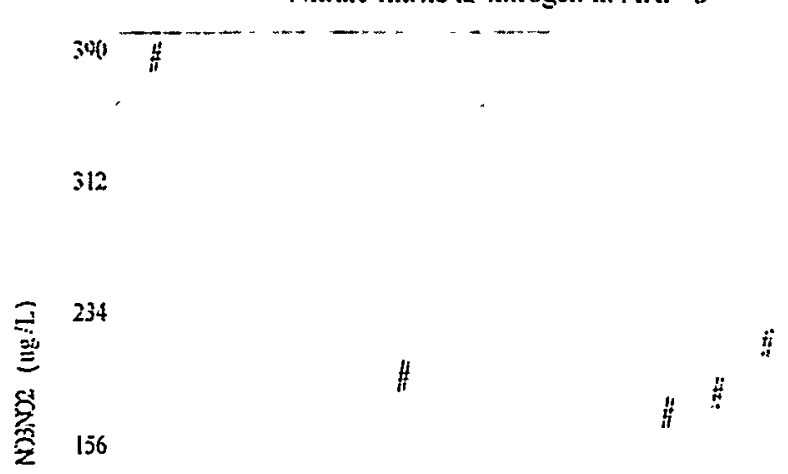

78

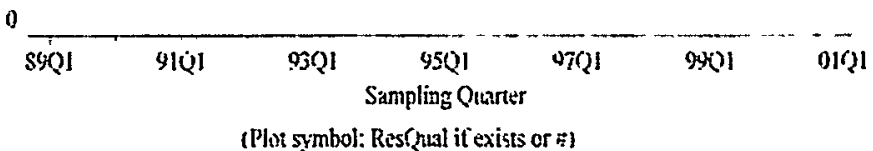


Selenium, total recoverable in ARP 3

Data Retrieved by AWSA v1 from GIMS on 09NOV99 at 15:50

Well Analyte Sample_Q Res_Qual Result Units

ARP 3 SETOT 1993Q1 U 2 UGL

ARP 3 SETOT 1995Q4 U 5 UGL

ARP 3 SETOT 1995Q4 U

ARP 3 SETOT 1997Q4 U 5 UGL

ARP 3 SETOT 1998Q3 U . 5 UGL

ARP 3 SETOT 1999Q3 U 5 UGL
Selenium, total recoverable in ARP :

U

总

) $\cdot$

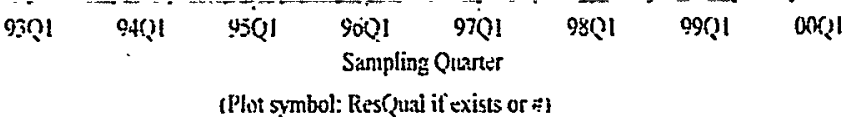


trans-1,2-Dichloroethylene in ARP 3

Data Retrieved by AWSA v1 from GIMS on 09NOV99 at 15:50

$\begin{array}{llll}\text { Well } & \text { Analyte } & \text { Sample_Q Re } \\ & & & \\ \text { ARP 3 } & \text { T12DCE } & 1985 Q 4 & \\ \text { ARP 3 } & \text { T12DCE } & 1988 Q 3 & \\ \text { ARP 3 } & \text { T12DCE } & 1989 Q 1 & \\ \text { ARP 3 } & \text { T12DCE } & 1989 Q 2 & U \\ \text { ARP 3 } & \text { T12DCE } & 1989 Q 3 & U \\ \text { ARP 3 } & \text { T12DCE } & 1989 Q 3 & U \\ \text { ARP 3 } & \text { T12DCE } & 1989 Q 4 & \\ \text { ARP 3 } & \text { T12DCE } & 1990 Q 1 & U \\ \text { ARP 3 } & \text { T12DCE } & 1990 Q 2 & U \\ \text { ARP 3 } & \text { T12DCE } & 1990 Q 4 & \\ \text { ARP 3 } & \text { T12DCE } & 1991 Q 1 & \\ \text { ARP 3 } & \text { T12DCE } & 1991 Q 1 & U \\ \text { ARP 3 } & \text { T12DCE } & 1991 Q 4 & U \\ \text { ARP 3 } & \text { T12DCE } & 1992 Q 1 & U \\ \text { ARP 3 } & \text { T12DCE } & 1992 Q 2 & \\ \text { ARP 3 } & \text { T12DCE } & 1993 Q 1 & U \\ \text { ARP 3 } & \text { T12DCE } & 1993 Q 2 & U \\ \text { ARP 3 } & \text { T12DCE } & 1993 Q 3 & U \\ \text { ARP 3 } & \text { T12DCE } & 1997 Q 4 & U \\ \text { ARP 3 } & \text { T12DCE } & 1998 Q 3 & U \\ \text { ARP 3 } & \text { T12DCE } & 1999 Q 3 & U\end{array}$$$
\text { UGL }
$$

trans-1.2-Dichloroethylene in ARP 3

$$
\begin{array}{r}
50 \text { UGL } \\
51.9 \text { UGL } \\
10 \text { UGL } \\
1 \text { UGL } \\
10 \text { UGL } \\
70 \text { UGL } \\
1 \text { UGL } \\
10 \text { UGL } \\
76.3 \text { UGL } \\
64.4 \text { UGL } \\
1 \text { UGL } \\
10 \text { UGL } \\
50 \text { UGL } \\
10.5 \text { UGL } \\
5 \text { UGL } \\
5 \text { UGL } \\
5 \text { UGL } \\
1 \text { UGL } \\
1 \text { UGL } \\
1 \text { UGL }
\end{array}
$$

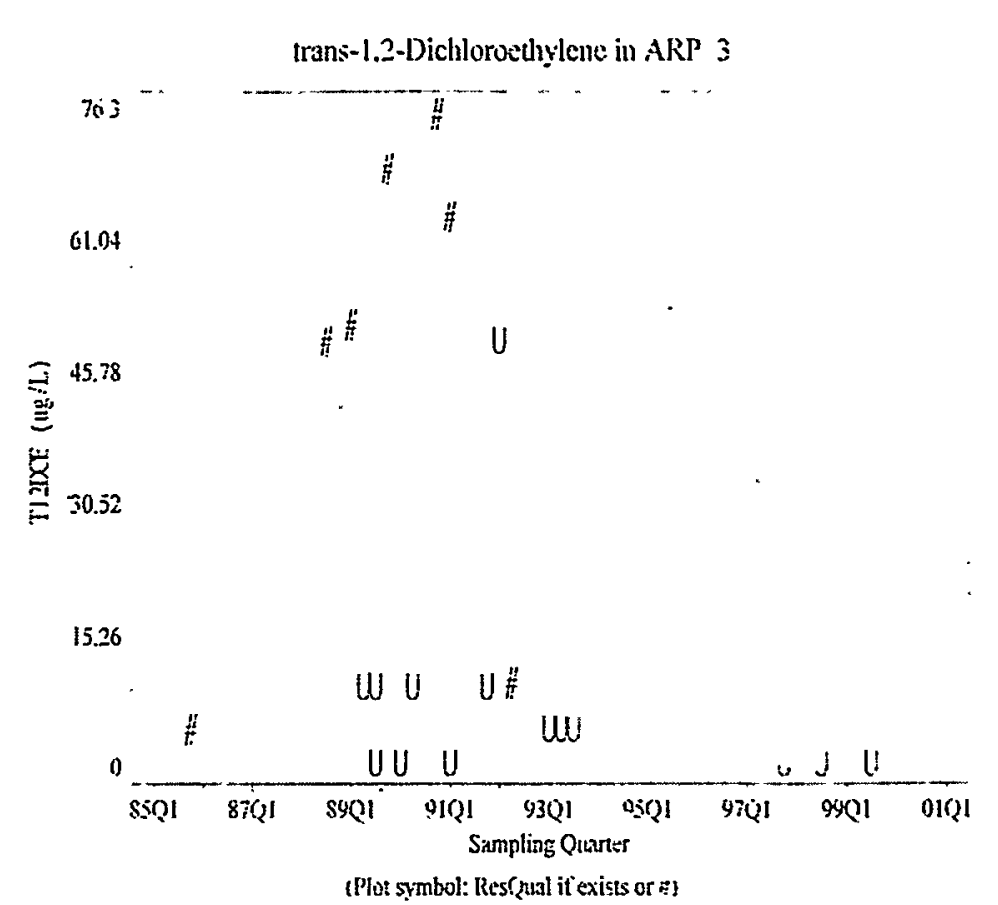


1,1,2,2-Tetrachloroethane in ARP 3

Data Retrieved by AWSA v1 from GIMS on 09NOV99 at 15:50

\begin{tabular}{llllr} 
Well & Analyte & \multicolumn{2}{c}{ Sample_Q Res_Qual Result } & Units \\
& & & & 10 UGL \\
ARP 3 & TCLEA & $1985 Q 4$ & & 1 UGL \\
ARP 3 & TCLEA & $1989 Q 3$ & $U$ & 10 UGL \\
ARP 3 & TCLEA & $1989 Q 3$ & $U$ & 1 UGL \\
ARP 3 & TCLEA & $1990 Q 1$ & $U$ & 1 UGL \\
ARP 3 & TCLEA & 199101 & $U$ & 5 UGL \\
ARP 3 & TCLEA & $1993 Q 1$ & $U$ & 5 UGL \\
ARP 3 & TCLEA & $1993 Q 2$ & $U$ & 5 UGL \\
ARP 3 & TCLEA & $1993 Q 3$ & $U$ & 0.5 UGL \\
ARP 3 & TCLEA & $1995 Q 4$ & $U$ & 0.5 UGL \\
ARP 3 & TCLEA & $1995 Q 4$ & UJ & 1 UGL \\
ARP 3 & TCLEA & $1997 Q 4$ & $U$ & 1 UGL \\
ARP 3 & TCLEA & 199803 & $U$ & 1 UGL \\
ARP 3 & TCLEA & $1999 Q 3$ & $U$ &
\end{tabular}

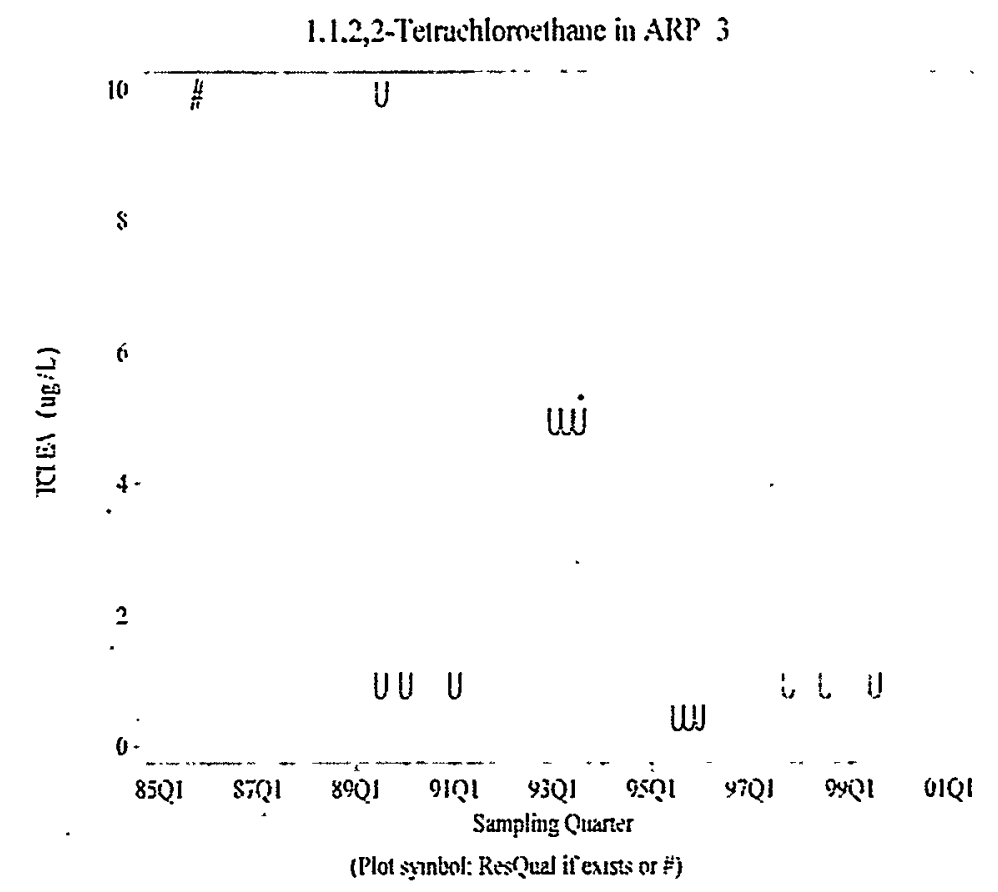


Zinc, tolal recoverable in ARP 3

Data Retrieved by AWSA v1 from GIMS on 23NOV99 at 08:45

Well Analyte Sample_Q Res_Qual Result Units

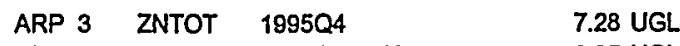

$\begin{array}{lllll}\text { ARP } 3 & \text { ZNTOT } & 1995 Q 4 & U & 8.85 \mathrm{UGL}\end{array}$

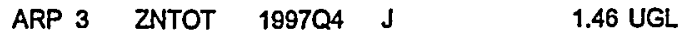

ARP 3 ZNTOT 1998Q3 U 5 UGL

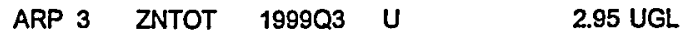

$\begin{array}{llll}\text { ARP } 3 & \text { ZNTOT } 1999 Q 3 & U & 2.95 \text { UGL }\end{array}$

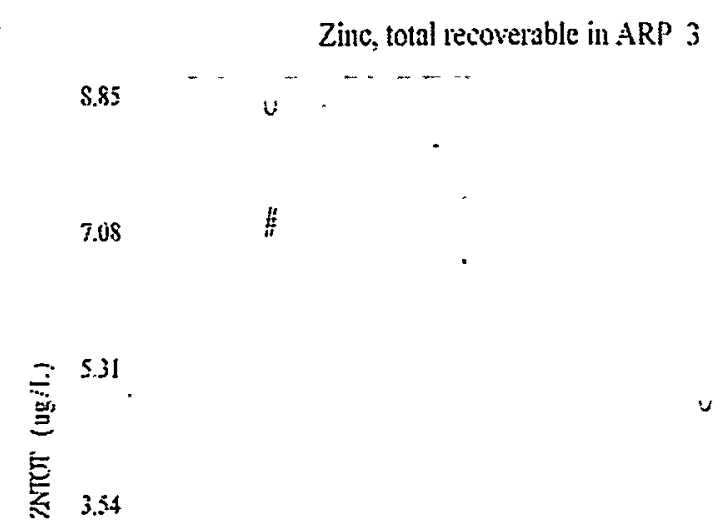

1.77

0 -

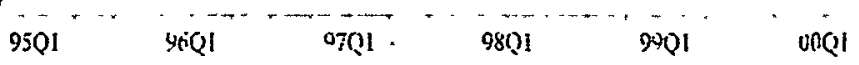
(Plot symhol: ResQual if exists ur :ै) 


\section{WELL BGO-33C DATA}


Field pH in BGO 33C

Data Retrioved by AWSA v1 from GIMS on 09NOV99 at 16:22

\begin{tabular}{|c|c|c|c|}
\hline Well & Analyte & Sample_Q Res_Qual Result & Units \\
\hline BGO $33 \mathrm{C}$ & FPH & $1989 Q 2$ & 5.8 PH \\
\hline BGO 33C & FPH & 198903 & 6.4 PH \\
\hline BGO 33C & FPH & $1989 Q 4$ & $6 \mathrm{PH}$ \\
\hline BGO $33 \mathrm{C}$ & FPH & $1990 Q 1$ & $6.4 \mathrm{PH}$ \\
\hline BGO $33 \mathrm{C}$ & FPH & 1990Q1 & $6.4 \mathrm{PH}$ \\
\hline BGO $33 \mathrm{C}$ & FPH & 1990Q2 & $5.4 \mathrm{PH}$ \\
\hline BGO $33 \mathrm{C}$ & FPH & $1990 Q 2$ & $5.4 \mathrm{PH}$ \\
\hline BGO 33C & FPH & 1990Q3 & 5.6 PH \\
\hline BGO 33C & FPH & 1990Q3 & 5.6 PH \\
\hline BGO 33C & FPH & $1990 Q 4$ & $6.1 \mathrm{PH}$ \\
\hline BGO 33C & FPH & 1991Q1 & 5.8 PH \\
\hline BGO 33C & FPH & 1991Q2 & $5.9 \mathrm{PH}$ \\
\hline BGO 33C & FPH & 1991Q3 & $5.7 \mathrm{PH}$ \\
\hline BGO $33 \mathrm{C}$ & FPH & 1991Q3 & $5.9 \mathrm{PH}$ \\
\hline BGO $33 \mathrm{C}$ & FPH & $1991 Q 4$ & $5.9 \mathrm{PH}$ \\
\hline BGO 33C & FPH & 1992Q1 & 5.4 PH \\
\hline BGO 33C & FPH & 1992Q1 & $5.4 \mathrm{PH}$ \\
\hline BGO $33 C$ & FPH & 199202 & $5.2 \mathrm{PH}$ \\
\hline BGO 33C & $\begin{array}{l}\text { FPH } \\
\text { FPH }\end{array}$ & 1992Q2 & $\begin{array}{l}5.2 \mathrm{PH} \\
5.6 \mathrm{PH}\end{array}$ \\
\hline BGO $33 \mathrm{C}$ & FPH & 1992Q4 & 5.3 $\mathrm{PH}$ \\
\hline BGO 33C & FPH & $1992 Q 4$ & $5.9 \mathrm{PH}$ \\
\hline BGO $33 \mathrm{C}$ & FPH & $1993 Q 1$ & 5.7 PH \\
\hline BGO $33 \mathrm{C}$ & FPH & 1993Q2 & $5.7 \mathrm{PH}$ \\
\hline BGO $33 \mathrm{C}$ & FPH & 1993Q3 & 5.6 PH \\
\hline BGO 33C & FPH & 1993Q4 & 5.6 PH \\
\hline BGO 33C & FPH & 1994Q1 & 5.7 PH \\
\hline BGO 33C & FPH & $1994 Q 2$ & $5.7 \mathrm{PH}$ \\
\hline BGO $33 \mathrm{C}$ & FPH & $1994 Q 3$ & 5.6 PH \\
\hline BGO $33 \mathrm{C}$ & FPH & 1994Q4 & 5.6 PH \\
\hline BGO $33 \mathrm{C}$ & FPH & 1995Q1 & 5.6 PH \\
\hline BGO $33 C$ & FPH & 1995Q4 & 5.3 PH \\
\hline BGO $33 \mathrm{C}$ & FPH & 1996Q4 & 5.4 PH \\
\hline BGO 33C & FPH & $1997 Q 4$ & $5.4 \mathrm{PH}$ \\
\hline BGO 33C & FPH & $1997 Q 4$ & $5.4 \mathrm{PH}$ \\
\hline BGO $33 \mathrm{C}$ & FPH & 1998Q2 & 5.2 PH \\
\hline BGO $33 \mathrm{C}$ & FPH & 1998Q4 & $5 \mathrm{PH}$ \\
\hline BGO $33 \mathrm{C}$ & FPH & $1998 Q 4$ & $5.2 \mathrm{PH}$ \\
\hline BGO 33C & $\mathrm{FPH}$ & 1999Q3 & $5.4 \mathrm{PH}$ \\
\hline $\mathrm{BGO} 33 \mathrm{C}$ & FPH & 1999Q3 & 5.4 PH \\
\hline
\end{tabular}

Field pH in $B G O 33 \mathrm{C}$
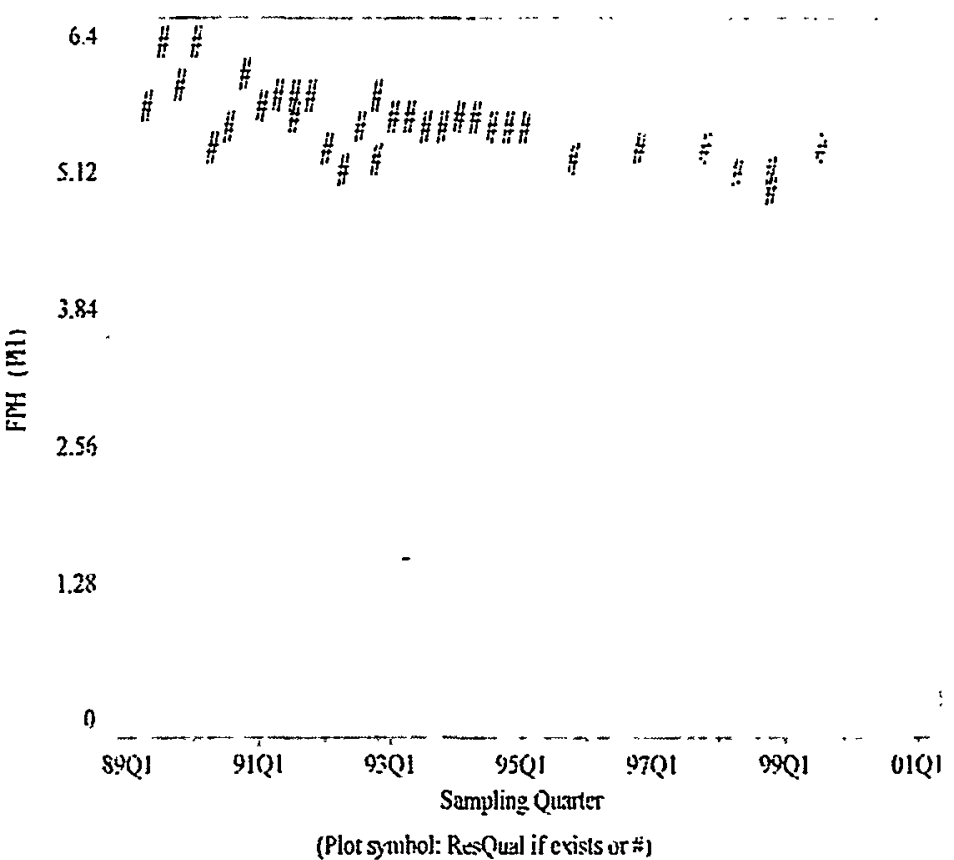

(Plot symhol: Res(qual if erists or 
Specific Conductance in BGO $33 \mathrm{C}$

Data Retrieved by AWSA v1 from GIMS on 09NOV99 at 16:22

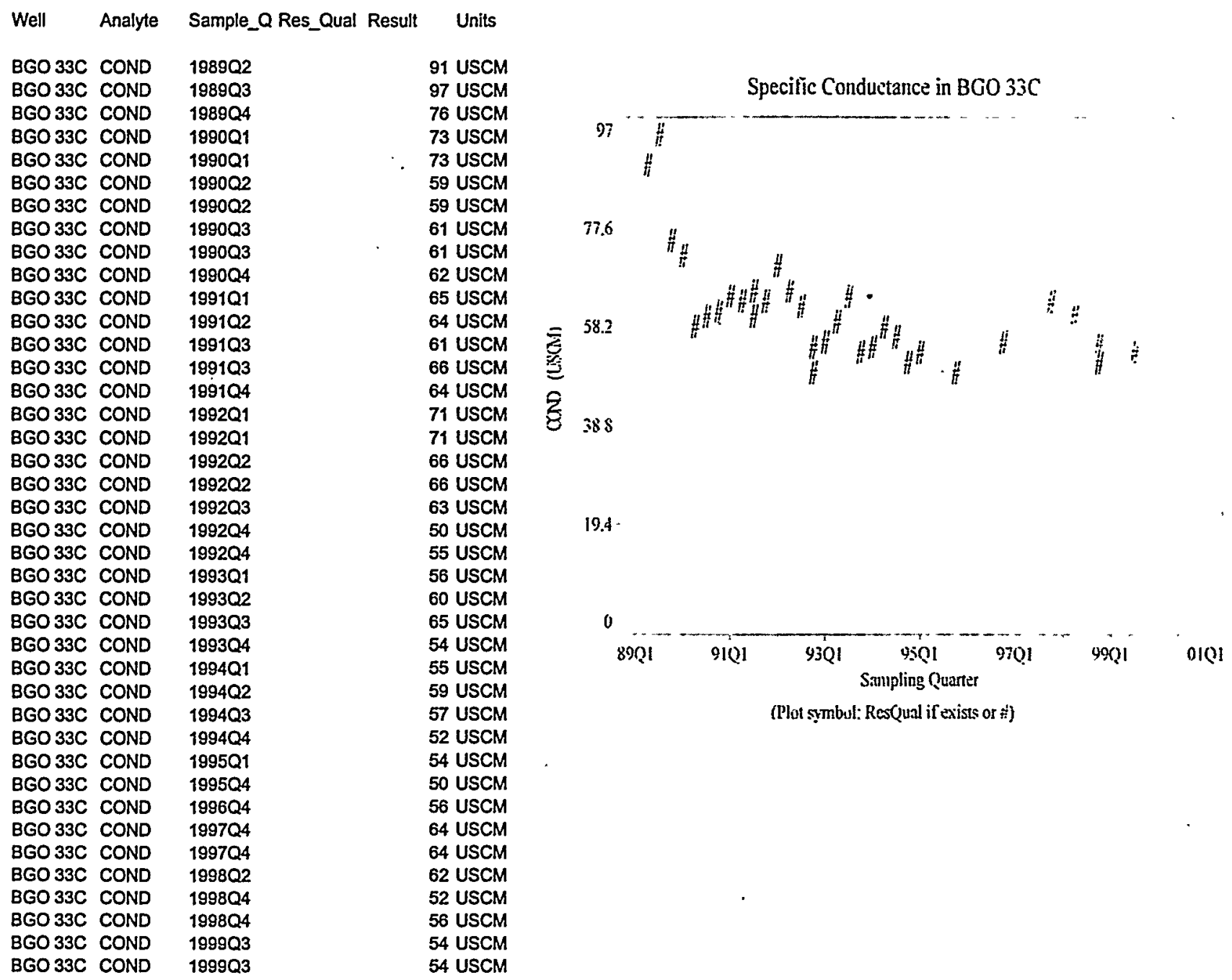


Calcium, total recoverable in $\mathrm{BGO} 33 \mathrm{C}$

Data Retrieved by AWSA v1 from GIMS on 09NOV99 at 15:50

Well Analyte Sample_QRes_Qua! Result Units

BGO 33C CATOT 199204 BGO $33 \mathrm{C}$ CATOT 1993Q1 $4760^{\circ} \mathrm{UGL}$ BGO 33C CATOT 199302 J 4680 UGL BGO 33C CATOT 1993Q3 6740 UGL BGO 33C CATOT 1993Q3 $6910 \mathrm{UGL}$ BGO 33C CATOT 1993Q4 4610 UGL BGO 33C CATOT 1994Q1 4240 UGL BGO 33C CATOT 19940224370 UGL BGO 33C CATOT 1994Q3 4250 UGL BGO 33C CATOT $199404 \quad 4000$ UGL BGO 33C CATOT 1994Q4 4070 UGL BGO 33C CATOT 1994Q4 $4610 \mathrm{UGL}$ BGO 33C CATOT 1994Q4 BGO 33C CATOT 1994Q4 BGO 33C CATOT $1995 Q 1$ BGO 33 C CATOT $1998 Q 2$ BGO 33C CATOT 1998Q4 BGO 33C CATOT 1999Q3
4610 UGL

4770 UGL 4380 UGL 5280 UGL 2970 UGL 3610 UGL

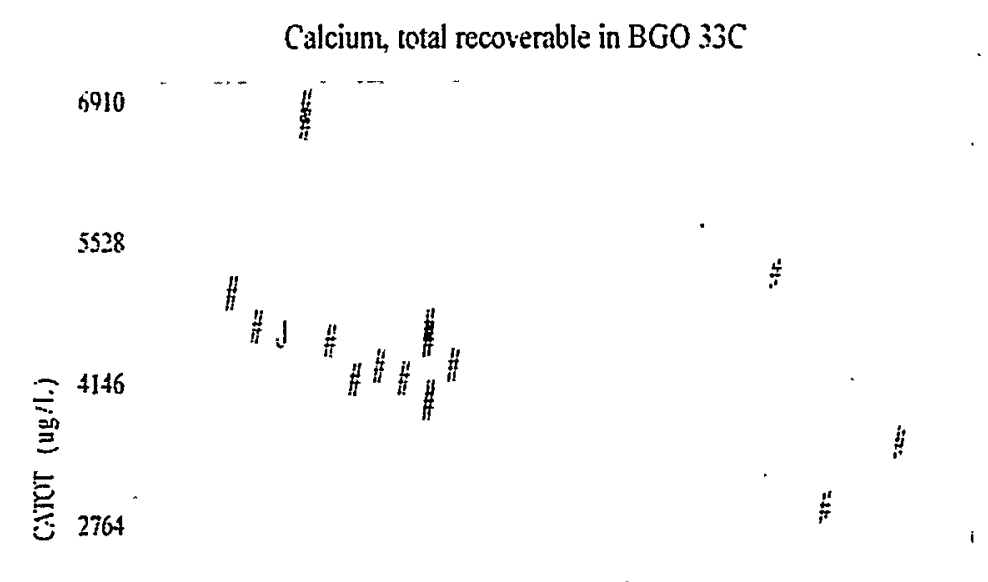

1382

0

9201 9301 9401 9501 9601 9701 9801 9001 0001

(Plor șinbol: Res(usal if exists or $\doteqdot$ ) 
Potassium, total recoverable in $\mathrm{BGO} 33 \mathrm{C}$

Data Retrieved by AWSA v1 from GIMS on 09NOV99 at 15:50

Well Analyte Sample_QRes_Qual Result Units

BGO 33C KTOT 1992Q4 U 500 UGL

BGO 33C KTOT 1993Q1 U 500 UGL

BGO 33C KTOT 1993Q2 U 500 UGL

BGO 33C KTOT $199303 \quad 511$ UGL

BGO 33C KTOT 1993Q3 534 UGL

BGO 33C KTOT 1993Q4 U 500 UGL

BGO 33C KTOT $1994 Q 1 \mathrm{~J} \quad 504 \mathrm{UGL}$

BGO 33C KTOT $1994 Q 2 \cup \quad 500$ UGL

BGO 33C KTOT $1994 Q 3 \quad 596$ UGL

BGO 33C KTOT 1994Q4 J 533 UGL

BGO 33C KTOT $1994 Q 4$ U 500 UGL

BGO 33C KTOT 1994Q4 U 500 UGL

BGO 33C KTOT $1994 Q 4 U$ U 500 UGL

BGO 33C KTOT 1994Q4 U 500 UGL

BGO 33C KTOT 199501 J

BGO 33C KTOT $199802 \quad 538$ UGL

BGO 33C KTOT $198804 \quad 419$ UGL

BGO 33C KTOT $1999 Q 3 \quad 449$ UGL

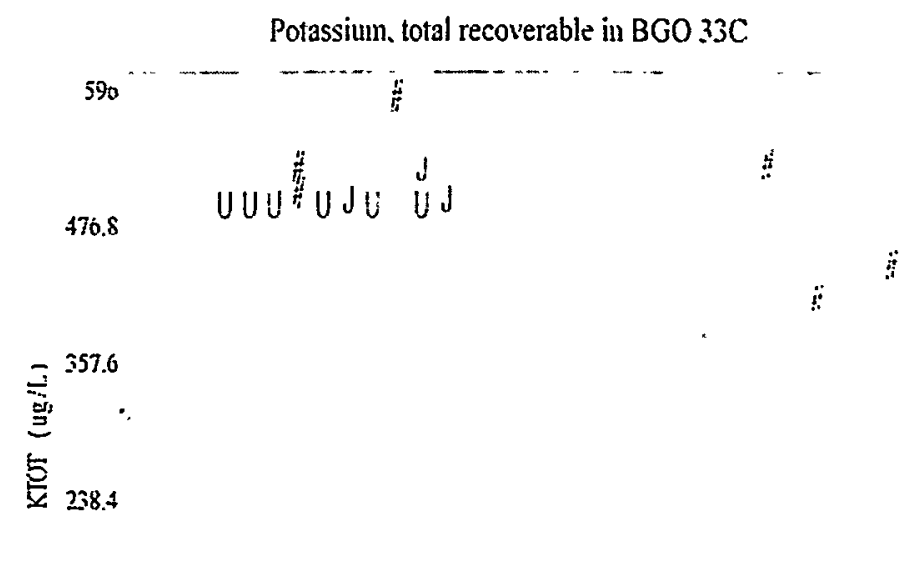

$119.2-$

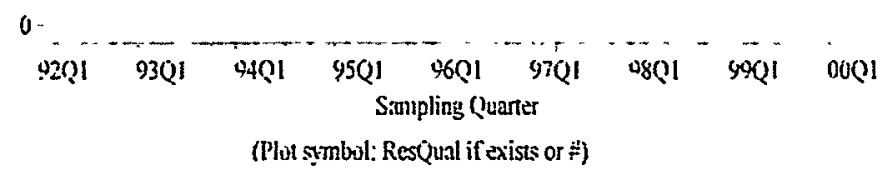


Sodium, total recoverable in BGO $33 \mathrm{C}$

Data Retrieved by AWSA v1 from GIMS on 09NOV99 at 15:50

Well Analyte Sample_Q Res_Qual Result Units

BGO 33C NATOT 199204

BGO 33C NATOT 1993Q1

BGO 33C NATOT 199302

BGO 33C NATOT 199303

BGO 33C NATOT 199303

BGO $33 C$ NATOT $1993 Q 4$

BGO 33C NATOT 199401

BGO 33C NATOT 199402

BGO 33C NATOT 199403

BGO 33C NATOT 1994Q4

BGO 33C NATOT 199404

BGO 33C NATOT 1994Q4

BGO 33C NATOT 199404

BGO 33C NATOT 1994Q4

BGO 33C NATOT 199501

BGO 33C NATOT 199802

BGO 33C NATOT 1998Q4

BGO 33 C NATOT $1999 Q 3$
3180 UGL 3310 UGL 3350 UGL 3270 UGL 3380 UGL 3110 UGL 3250 UGL 3280 UGL 3280 UGL 3370 UGL 3390 UGL 3400 UGL 3400 UGL 3420 UGL 3440 UGL 4610 UGL 3710 UGL 4210 UGL

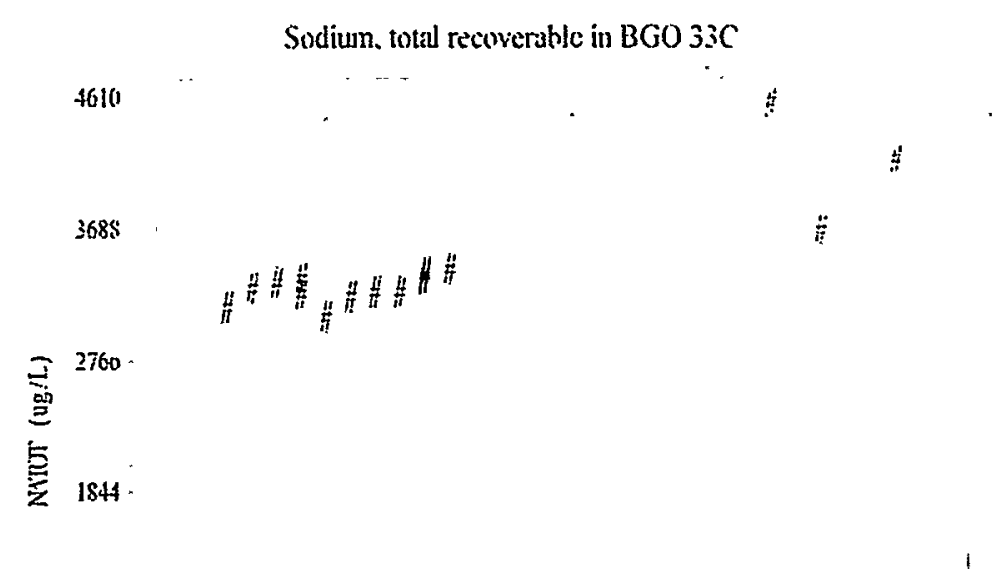

922

1)

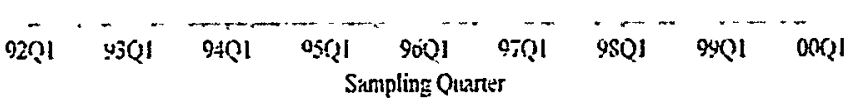

(Plot symbol: Res(yual if exists or at? 
Chromium, total recoverable in 8 GO $33 \mathrm{C}$

Data Retrieved by AWSA v1 from GIMS on 09NOV99 at 15:50

\begin{tabular}{|c|c|c|c|c|c|}
\hline Well & Analyte & Sample_c & Res_Qual & Result & Units \\
\hline BGO 33C & CRTOT & $1992 Q 4$ & $u$ & & 4 UGL \\
\hline $\mathrm{BGO} 33 \mathrm{C}$ & CRTOT & 199301 & $U$ & & 4 UGL \\
\hline BGO $33 \mathrm{C}$ & CRTOT & 199302 & $u$ & & 4 UGL \\
\hline BGO 33C & CRTOT & $1993 Q 3$ & $u$ & & 4 UGL \\
\hline BGO $33 \mathrm{C}$ & CRTOT & $1993 Q 3$ & $U$ & & 4 UGL \\
\hline BGO 33C & CRTOT & 199304 & $U$ & & $4 \mathrm{C}$ \\
\hline BGO 33C & CRTOT & 1994Q1 & $u$ & & 4 UG \\
\hline BGO $33 \mathrm{C}$ & CRTOT & 199402 & $U$ & & 4 UGL \\
\hline BGO 33C & CRTOT & 1994Q3 & U & & 4 UGL \\
\hline BGO 33C & CRTOT & 1994Q4 & U & & 4 UGL \\
\hline $8 G 033 C$ & CRTOT & $1994 Q 4$ & $U$ & & 4 UGL \\
\hline BGO $33 \mathrm{C}$ & CRTOT & $1994 Q 4$ & $U$ & & 4 UGL \\
\hline BGO $33 \mathrm{C}$ & CRTOT & $1994 Q 4$ & $U$ & & 4 UGL \\
\hline BGO $33 \mathrm{C}$ & CRTOT & $1994 Q 4$ & $U$ & & 4 UGL \\
\hline BGO $33 \mathrm{C}$ & CRTOT & $1995 Q 1$ & $u$ & & 3.67 UGL \\
\hline BGO 33C & CRTOT & $1995 Q 4$ & $J$ & & 1.18 UGL \\
\hline BGO 33C & CRTOT & $1996 Q 4$ & U & & 2.29 UGL \\
\hline BGO 33C & CRTOT & $1997 Q 4$ & $\mathrm{~J}$ & & 2.93 UGL \\
\hline BGO 33C & CRTOT & $1988 Q 2$ & $J$ & & 2.02 UGL \\
\hline BGO 33C & CRTOT & $1998 Q 4$ & $J$ & & 1.46 UGL \\
\hline $8 G 033 C$ & CRTOT & $1998 Q 4$ & $J$ & & 2.1 UGL \\
\hline BGO 33C & CRTOT & $1999 Q 3$ & $J$ & & $1.98 \mathrm{~L}$ \\
\hline
\end{tabular}

Chromium, total recoverable in $\mathrm{BGO} 33 \mathrm{C}$

$6.6 ?$

\section{U}

5.330

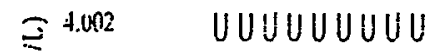

벌

2.6.8.

u

1.334

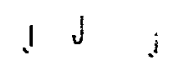

J

()

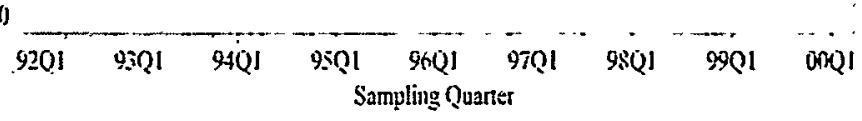

(Plnt șmbol: ResQual if exists or f) 
Iron, total recoverable in BGO $33 \mathrm{C}$

Data Retrieved by AWSA v1 from GIMS on 09NOV99 at 15:50

\begin{tabular}{|c|c|c|c|c|}
\hline Well & Analyte & Sample_c & Res_Qual Resul & Units \\
\hline BGO 33C & FETOT & $1992 Q 4$ & & 10.3 UGL \\
\hline BGO $33 \mathrm{C}$ & FETOT & 1993Q1 & & 12 UGL \\
\hline BGO 33C & FETOT & 199302 & $U$ & 4 UGL \\
\hline BGO 33C & FETOT & $1993 Q 3$ & & 4.95 UGL \\
\hline BGO 33C & FETOT & 199303 & & 5.03 UGL \\
\hline BGO $33 \mathrm{C}$ & FETOT & $1993 Q 4$ & & 7.74 UGL \\
\hline BGO 33C & FETOT & 1994Q1 & & 8.36 UGL \\
\hline BGO 33C & FETOT & $1994 Q 2$ & $J$ & 6.37 UGL \\
\hline $\mathrm{BGO} 33 \mathrm{C}$ & FETOT & $1994 Q 3$ & & 17.3 UGL \\
\hline BGO 33C & FETOT & $1994 Q 4$ & & 16.9 UGL \\
\hline BGO 33C & FETOT & 1994Q4 & & 39.3 UGL \\
\hline BGO $33 \mathrm{C}$ & FETOT & 1994Q4 & $\mathbf{J}$ & 33.8 UGL \\
\hline BGO 33C & FETOT & 1994Q4 & $\mathbf{u}$ & 4 UGL \\
\hline BGO 33C & FETOT & $1994 Q 4$ & $\mathbf{U}$ & 4 UGL \\
\hline BGO 33C & FETOT & $1995 Q 1$ & $\mathbf{J}$ & 5.96 UGL \\
\hline BGO 33C & FETOT & 198802 & $J$ & 32.1 UGL \\
\hline BGO 33C & FETOT & $1998 Q 4$ & $J$ & 35.8 UGL \\
\hline BGO 33C & FETOT & 199884 & $\mathbf{U}$ & 50 UGL \\
\hline $3 G 033 C$ & FETOT & $1999 Q 3$ & $\mathbf{U}$ & 50 UGL \\
\hline
\end{tabular}

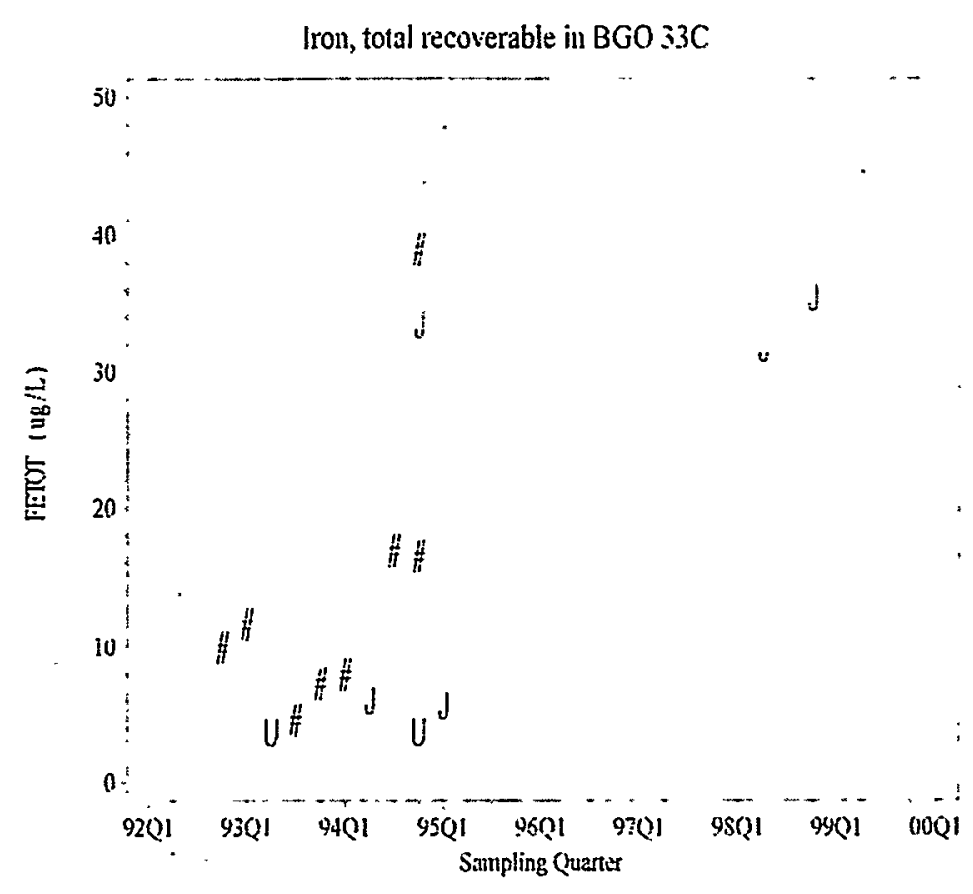

(Plot simbul: ResQual it exists or $\rightarrow$ ) 
Manganese, total recoverable in BGO $33 \mathrm{C}$

Data Retrieved by AWSA v1 from GIMS on 09NOV99 at 15:50

Well Analyte Sample_Q Res_Qual Result Units

BGO 33C MNTOT 19920

BGO 33C MNTOT 199301

BGO 33C MNTOT 199302

BGO 33C MNTOT 199303

BGO 33C MNTOT 199303

BGO 33C MNTOT 199304

BGO 33C MNTOT 199401

BGO 33C MNTOT 1994 Q2

BGO 33C MNTOT 199403

BGO 33C MNTOT 1994Q4

BGO 33C MNTOT 1994Q4

BGO 33C MNTOT 1994Q4

BGO 33C MNTOT 1994Q4

BGO 33C MNTOT 1994Q4

BGO 33C MNTOT 1995Q1

BGO 33C MNTOT $1998 Q 2$

BGO 33C MNTOT 1998Q4

BGO 33C MNTOT 1999Q3
31.8 UGL 30.2 UGL 29.2 UGL 32.5 UGL 33.3 UGL 29.3 UGL 29 UGL 30.2 UGL 32.1 UGL

31.1 UGL 31.3 UGL 33.1 UGL 33.6 UGL 33.8 UGL 34 UGL 45.3 UGL 45.4 UGL 48.3 UGL

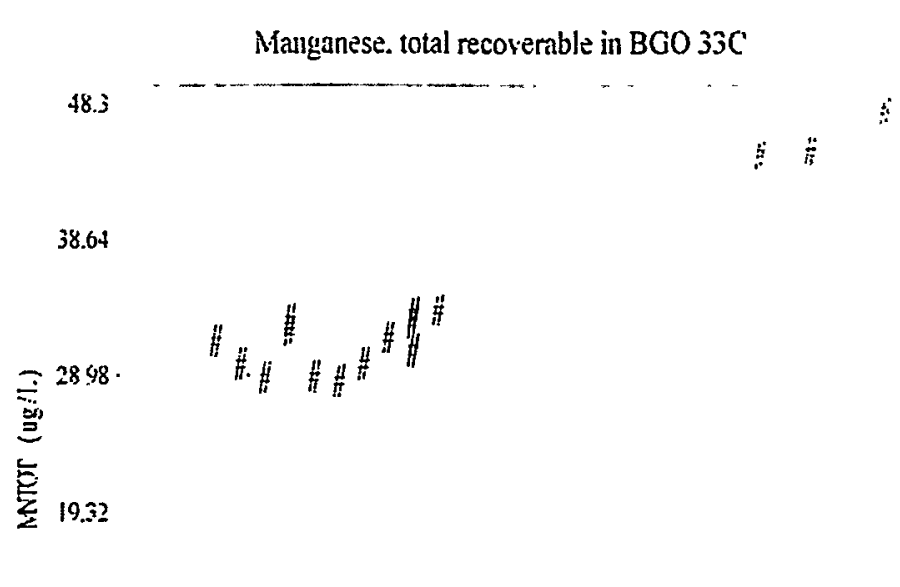

9.66 
Aluminum, total recoverable in $\mathrm{BGO} 33 \mathrm{C}^{\circ}$

Data Retrieved by AWSA v1 from GIMS on 09NOV99 at 15:50

Well Analyte Sample_QRes_Qual Result Units

BGO 33C ALTOT 199204

BGO 33C ALTOT 199301

BGO 33C ALTOT 199302

BGO 33C ALTOT 1993Q3

BGO 33C ALTOT 1993Q3

BGO 33C ALTOT 1993Q4

BGO 33C ALTOT 199401

BGO 33C ALTOT 1994Q2

BGO 33C ALTOT 1994Q3

BGO 33C ALTOT 1894Q4

BGO 33C ALTOT 1994Q4

BGO 33C ALTOT 1994Q4

BGO 33C ALTOT 1994Q4

BGO 33C ALTOT 1994Q4

BGO 33C ALTOT 1995Q1

BGO 33C ALTOT $1998 Q 2$

BGO 33C ALTOT 1998Q4 J

BGO 33C ALTOT 1998Q4 J

BGO 33C ALTOT 1999Q3
61 UGL 72.5 UGL 72.3 UGL 180 UGL 186 UGL 659 UGL 251 UGL 38.3 UGL 90.1 UGL 72.8 UGL 81.6 UGL 86.2 UGL 88.9 UGL 94 UGL 32.2 UGL 46.1 UGL 40.1 UGL 58.1 UGL 57.4 UGL
Aluminum. total recoverable in BGO $33 \mathrm{C}$

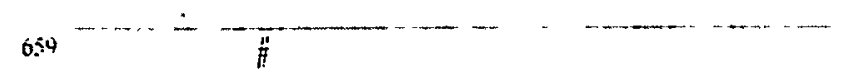

527.2

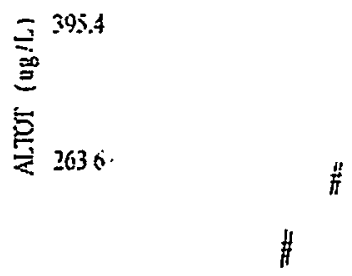

131.8 .

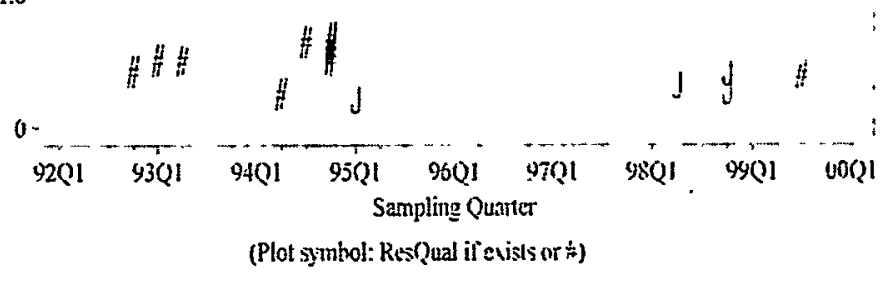


Lead, total recoverable in BGO $33 \mathrm{C}$

Data Retrieved by AWSA v1 from GIMS on 09NOV99 at 15:50

\begin{tabular}{|c|c|c|c|c|c|}
\hline Well & Analyte & Sample_Q & Res_Qual & Result & Units \\
\hline BGO 33C & РВTOT & $1992 Q 4$ & U & & 3 UGL \\
\hline BGO $33 \mathrm{C}$ & РВТОT & 1993Q1 & $u$ & & 3 UGL \\
\hline $\mathrm{BGO} 33 \mathrm{C}$ & РВTOT & 199302 & $u$ & & 3 UGL \\
\hline BGO 33C & PBTOT & $1993 Q 3$ & $\mathbf{U}$ & & 3 UGL \\
\hline BGO $33 \mathrm{C}$ & PBTOT & $1993 Q 3$ & U & & 3 UGL \\
\hline BGO $33 \mathrm{C}$ & PBTOT & $1993 Q 4$ & $\mathbf{U}$ & & 3 UGL \\
\hline$B G O 33 C$ & PBTOT & 1994Q1 & $u$ & & 3 UGL \\
\hline BGO $33 \mathrm{C}$ & PBTOT & 1994Q2 & $u$ & & 3 UGL \\
\hline BGO $33 \mathrm{C}$ & PBTOT & 1994Q3 & $u$ & & 3 UGL \\
\hline BGO $33 \mathrm{C}$ & РВTOT & $1994 Q 4$ & $u$ & & 3 UG \\
\hline BGO $33 \mathrm{C}$ & PBTOT & $1994 Q 4$ & $u$ & & 3 UGL \\
\hline BGO 33C & PBTOT & $1994 Q 4$ & $U$ & & 3 UGL \\
\hline BGO $33 \mathrm{C}$ & РВTOT & $1994 Q 4$ & $\mathbf{U}$ & & 3 UGL \\
\hline BGO $33 \mathrm{C}$ & PBTOT & 1994Q4 & U & & 3 UGL \\
\hline BGO 33C & РВTOT & 1995Q1 & $u$ & & 5 UGL \\
\hline $\mathrm{BGO} 33 \mathrm{C}$ & РBTOT & 1995Q1 & $u$ & & 5 UGL \\
\hline BGO $33 \mathrm{C}$ & РВTOT & $1995 Q 4$ & $u$ & & 5 UGL \\
\hline $\mathrm{BGO} 33 \mathrm{C}$ & PBTOT & $1996 Q 4$ & $u$ & & 2.54 UGL \\
\hline BGO $33 \mathrm{C}$ & PBTOT & $1997 Q 4$ & $u$ & & 5 UGL \\
\hline BGO 33C & PBTOT & 199802 & $J$ & & 3.07 UGL \\
\hline BGO 33C & PBTOT & $1998 Q 4$ & U & & 5 UGL \\
\hline GO 33C & РВTOT & $1998 Q 4$ & $U$ & & 47 UGL \\
\hline GO 33C & PBTOT & 1999Q3 & $U$ & & 5 UG \\
\hline
\end{tabular}

Lead. total recoverable in $\mathrm{BC}(\mathrm{O}) \mathrm{i3C}$

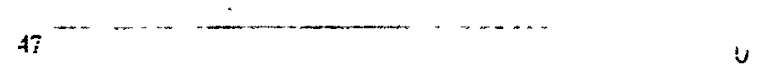

376.

$=28.2$

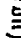

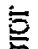

$15.8-$

6.4-

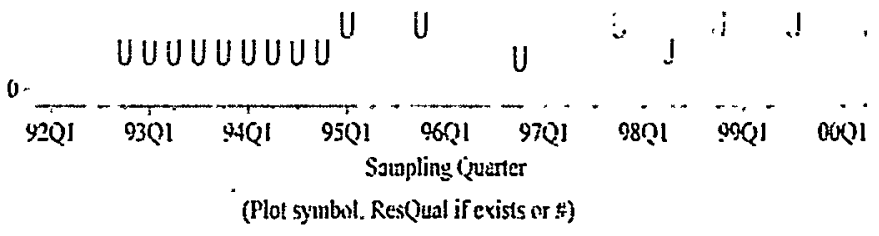


Chloride in BGO $33 \mathrm{C}$

Data Retrieved by AWSA v1 from GIMS on 09NOV99 at 15:50

Well Analyte Sample_QRes_Qual Result Units

BGO 33C CL 198902

BGO $33 \mathrm{C} \mathrm{CL} \quad 1989 \mathrm{CL}$

BGO 33C CL $1989 Q 4$

BGO 33C CL $1990 \mathrm{CL}$

BGO 33C CL 199022

BGO 33C CL $\quad 199022$

BGO 33C CL $\quad 199002$

BGO $33 \mathrm{C} \mathrm{CL} \quad 199002$

BGO 33C CL $\quad 199003$

BGO 33C CL 1990 Q 3

BGO 33C CL $1990 Q 4$

BGO 33C CL 1991Q1

BGO 33C CL $\quad .1991 Q 2$

BGO 33C CL 199102

BGO 33C CL 1991Q3

BGO 33C CL $1991 Q 4$

BGO 33C CL $1992 \mathrm{CL}$

BGO $33 \mathrm{C} \mathrm{CL} \quad 1992 \mathrm{Q} 2$

BGO 33C CL $1992 Q 3$

BGO 33C CL $1992 Q 4$

BGO 33C CL $1993 \mathrm{Cl}$

BGO 33C CL 199302

$86033 \mathrm{C} \mathrm{CL} \quad 199322$

BGO 33C CL $1993 Q 3$

BGO $33 \mathrm{C} \mathrm{CL} \quad 1993 \mathrm{Q} 3$

BGO 33C CL $1993 Q 4$

BGO 33C CL $1994 Q 1$

BGO 33C CL 199402

BGO 33C CL $\quad 1994$ Q2

BGO 33C CL $1994 Q 3$

BGO 33C CL 1994Q4

BGO 33C CL $1994 Q 4$

BGO 33C CL 1994Q4

BGO 33C CL 199404

BGO 33C CL 1994Q4

BGO 33C CL 1995Q1

BGO 33C CL 199802

BGO 33C CL $1998 Q 2$

BGO 33C CL $1998 Q 4$

BGO 33C CL $1998 Q 4$

BGO 33C CL 199903

BGO 33C CL 1999 Q 3
4100 UGL

4500 UGL

4800 UGL

4000 UGL

4200 UGL

4400 UGL

5000 UGL

5000 UGL

5410 UGL

5420 UGL

5080 UGL

5090 UGL

4950 UGL

5200 UGL

6300 UGL

5640 UGL

5500 UGL

5440 UGL

5240 UGL

5400 UGL

5890 UGL

5420 UGL

5520 UGL

5570 UGL

5720 UGL

5710 UGL

5420 UGL

5970 UGL

5990 UGL

1450 UGL

5200 UGL

5210 UGL

5300 UGL

5890 UGL

6070 UGL

5480 UGL

8350 UGL

8520 UGL

7700 UGL

7720 UGL

7520 UGL

7690 UGL

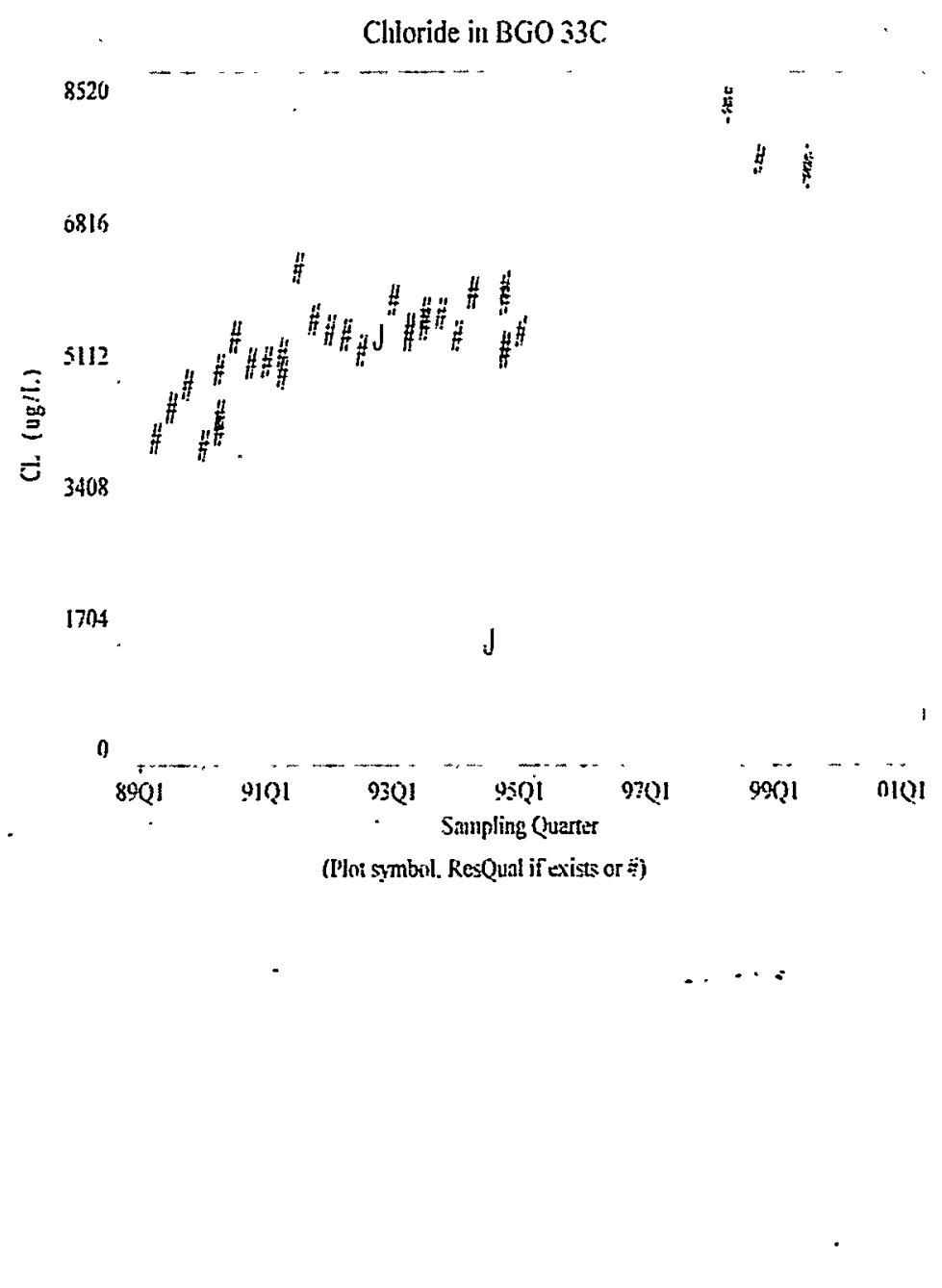


Carton tetrachloride in BGO $33 \mathrm{C}$

Data Retrieved by AWSA v1 from GIMS on 09NOV99 at 15:50

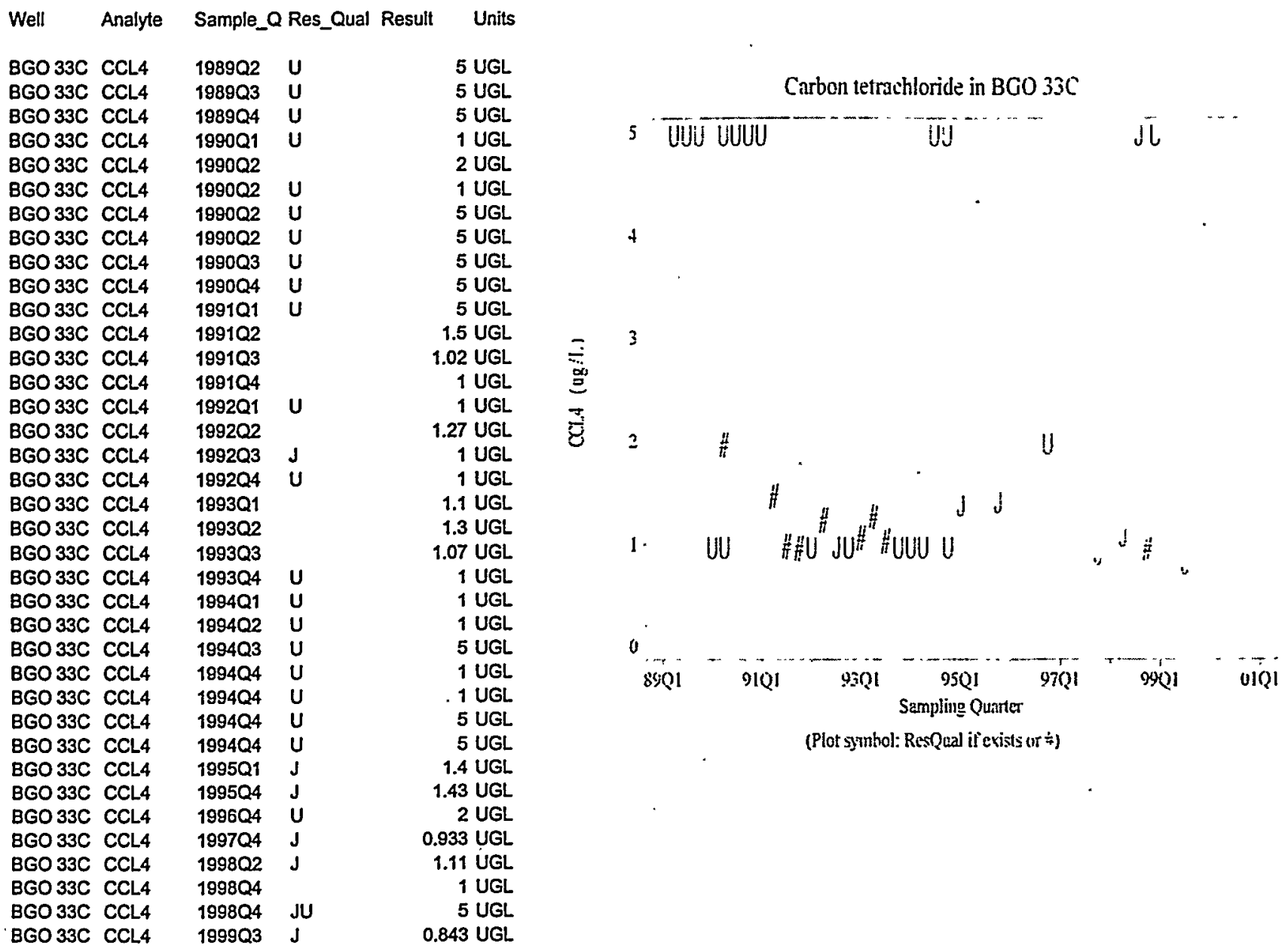


Tetrachloroethylene in BGO $33 \mathrm{C}$

Data Retrieved by AWSA v1 from GIMS on 09NOV99 at 15:50

\begin{tabular}{|c|c|c|c|c|}
\hline Well & Analyte & Sample_ & Res_Qual & Result \\
\hline BGO 33C & TCLEE & 198902 & U & 5 UGL \\
\hline $\mathrm{BGO} 33 \mathrm{C}$ & TCLEE & 1989Q3 & $J$ & 4 UGL \\
\hline BGO 33C & TCLEE & $1989 Q 4$ & $u$ & 5 UGL \\
\hline BGO $33 \mathrm{C}$ & TCLEE & 1990Q1 & & 3 UGL \\
\hline BGO $33 \mathrm{C}$ & TCLEE & $1990 Q 2$ & & 3 UGL \\
\hline GO 33C & TCLEE & 199002 & & 6 UGL \\
\hline BGO $33 \mathrm{C}$ & TCLEE & $1990 Q 2$ & $J$ & 3 UGL \\
\hline BGO $33 \mathrm{C}$ & TCLEE & 1990Q2 & $J$ & 3 UGL \\
\hline Go 33C & TCLEE & $1990 Q 3$ & $J$ & 3 UGL \\
\hline GO 33C & TCLEE & $1990 Q 4$ & & 5 UGL \\
\hline BGO 33C & TCLEE & 1991Q1 & $J$ & 3 UGL \\
\hline GO 33C & TCLEE & 1991Q2 & & 5.4 UGL \\
\hline BGO 33C & TCLEE & 1991Q3 & & 3.24 UGL \\
\hline GO $33 \mathrm{C}$ & TCLEE & $1991 Q 4$ & & 4.1 UGL \\
\hline BGO $33 \mathrm{C}$ & TCLEE & $1992 Q 1$ & & 3.48 UGL \\
\hline BGO $33 \mathrm{C}$ & TCLEE & 199202 & & 4.97 UGL \\
\hline BGO $33 C$ & TCLEE & 1992Q3 & $J$ & 5.5 UGL \\
\hline BGO $33 \mathrm{C}$ & TCLEE & $1992 Q 4$ & & 4.1 UGL \\
\hline BGO $33 \mathrm{C}$ & TCLEE & 1993Q1 & & 4.3 UGL \\
\hline $\mathrm{BGO} 33 \mathrm{C}$ & TCLEE & $1993 Q 2$ & & 5.8 UGL \\
\hline $8 G 033 C$ & TCLEE & 1993Q3 & & 4.42 UGL \\
\hline BGO $33 \mathrm{C}$ & TCLEE & $1993 Q 4$ & & 4.4 UGL \\
\hline BGO 33C & TCLEE & 1994Q1 & & 5.1 UGL \\
\hline SO $33 \mathrm{C}$ & TCLEE & 1994Q2 & & 5.2 UGL \\
\hline BGO 33C & TCLEE & $1994 Q 3$ & $J$ & 4.52 UGL \\
\hline BGO 33C & TCLEE & $1994 Q 4$ & & 4 UGL \\
\hline BGO $33 \mathrm{C}$ & TCLEE & 1994Q4 & & 4.2 UGL \\
\hline $\mathrm{BGO} 33 \mathrm{C}$ & TCLEE & $1994 Q 4$ & & 4.74 UGL \\
\hline BGO $33 \mathrm{C}$ & TCLEE & 1994Q4 & & 4.98 UGL \\
\hline BGO 33C & TCLEE & 1995Q1 & & 5.7 UGL \\
\hline BGO 33C & TCLEE & 1995Q4 & & 5.34 UGL \\
\hline BGO $33 \mathrm{C}$ & TCLEE & 1996Q4 & & 6 UGL \\
\hline BGO $33 \mathrm{C}$ & TCLEE & 1997Q4 & & 5.98 UGL \\
\hline BGO $33 \mathrm{C}$ & TCLEE & $1998 Q 2$ & $\mathrm{~J}$ & 6.42 UGL \\
\hline $8 G 033 C$ & TCLEE & 199804 & & 6.58 UGL \\
\hline BGO $33 \mathrm{C}$ & TCLEE & $1998 Q 4$ & $J$ & 4.96 UGL \\
\hline
\end{tabular}

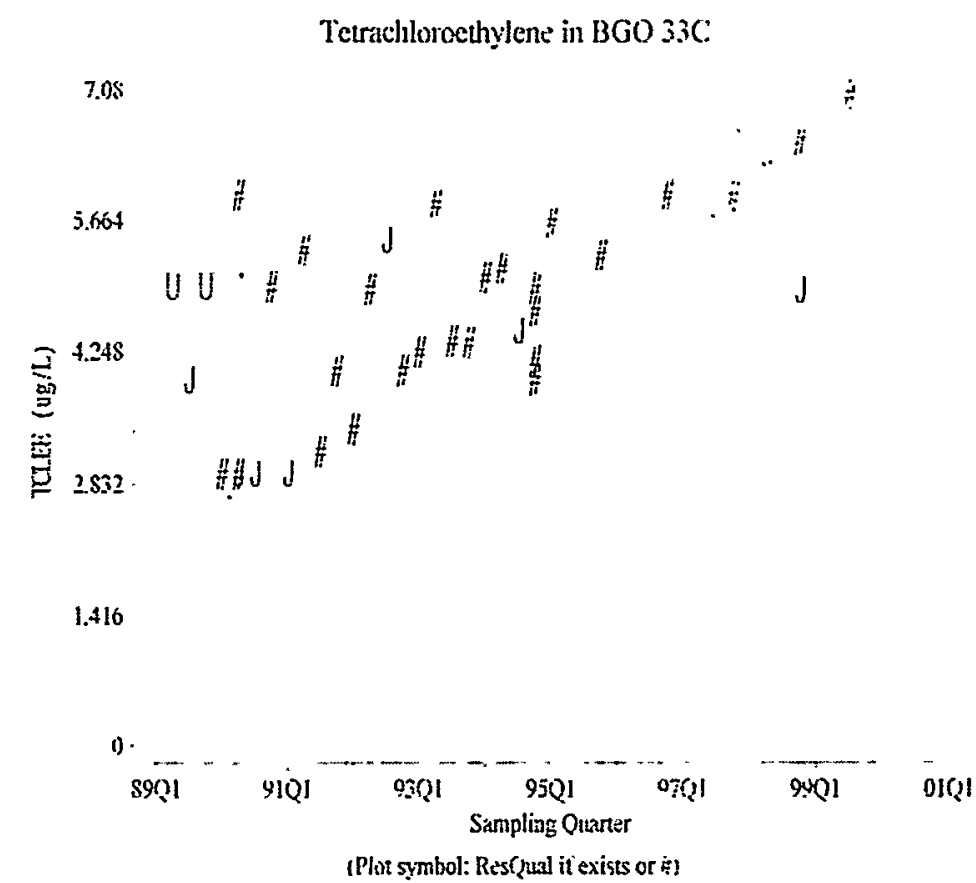


Trichloroethylene in BGO 33C

Data Retrieved by AWSA v1 from GIMS on 09NOV99 at 15:50

\begin{tabular}{|c|c|c|c|c|}
\hline Well & Analyte & Sample_c & 2 Res_Qual & Result \\
\hline BGO 33C & TRCLE & $1988 Q 2$ & & 11 UGL \\
\hline $36033 \mathrm{C}$ & TRCLE & 1989Q3 & & 37 UGL \\
\hline BGO 33C & TRCLE & 1989Q4 & & 33 UGL \\
\hline BGO 33C & TRCLE & $1990 Q 1$ & & $18 U$ \\
\hline BGO 33C & TRCLE & 199002 & & $15 U$ \\
\hline BGO $33 \mathrm{C}$ & TRCLE & $1990 Q 2$ & & 18 UG \\
\hline BGO $33 \mathrm{C}$ & TRCLE & 199002 & & 19 UGI \\
\hline BGO $33 \mathrm{C}$ & TRCLE & $1990 Q 2$ & & 19 UGL \\
\hline $8 G 033 C$ & TRCLE & $1990 Q 3$ & & 23 UGL \\
\hline BGO 33C & TRCLE & $1990 Q 4$ & & 15 UGL \\
\hline BGO 33C & TRCLE & 1991Q1 & & 18 UGL \\
\hline BGO $33 \mathrm{C}$ & TRCLE & 1991Q2 & & 23.3 UGL \\
\hline BGO $33 \mathrm{C}$ & TRCLE & 1991Q3 & & 16.8 UGL \\
\hline BGO $33 \mathrm{C}$ & TRCLE & 1991Q4 & & 19.5 UGL \\
\hline $86033 \mathrm{C}$ & TRCLE & 1992Q1 & & 18.4 UGL \\
\hline BGO 33C & TRCLE & 199202 & & 24.3 UGL \\
\hline BGO 33C & TRCLE & $1992 Q 3$ & $J$ & $24.3 \mathrm{UGL}$ \\
\hline BGO 33C & TRCLE & $1992 Q 4$ & & 22.4 UGL \\
\hline BGO 33C & TRCLE & $1993 Q 1$ & & 22.9 UGL \\
\hline $8 G O 33 \mathrm{C}$ & TRCLE & 1993Q2 & & 27 UGL \\
\hline BGO $33 \mathrm{C}$ & TRCLE & 199303 & & 28.1 UGL \\
\hline BGO 33C & TRCLE & $1993 Q 4$ & & 22.4 UGL \\
\hline BGO $33 \mathrm{C}$ & TRCLE & 1994Q1 & & 25.8 UGL \\
\hline BGO $33 \mathrm{C}$ & TRCLE & 1994Q2 & & 28 UGL \\
\hline BGO $33 \mathrm{C}$ & TRCLE & 1994Q3 & & 27.4 UGL \\
\hline $\mathrm{BGO} 33 \mathrm{C}$ & TRCLE & $1994 Q 4$ & & 24.4 UGL \\
\hline BGO 33C & TRCLE & 1994Q4 & & 24.9 UGL \\
\hline BGO 33C & TRCLE & $1994 Q 4$ & & 28.1 UGI \\
\hline BGO 33C & TRCLE & $1994 Q 4$ & & 29.6 UGL \\
\hline BGO 33C & TRCLE & 1995Q1 & . & 29.2 UGL \\
\hline BGO 33C & TRCLE & $1995 Q 4$ & & 25 UGL \\
\hline BGO $33 \mathrm{C}$ & TRCLE & $1996 Q 4$ & & 30.4 UGL \\
\hline $\mathrm{BGO} 33 \mathrm{C}$ & TRCLE & $1997 Q 4$ & & 54.1 UGL \\
\hline BGO 33C & TRCLE & $1998 Q 2$ & $J$ & 48.2 UGL \\
\hline BGO 33C & TRCLEE & $1998 Q 4$ & & 47.4 UGL \\
\hline BGO $33 \mathrm{C}$ & TRCLE & $1998 Q 4$ & $\mathbf{J}$ & 32 UGL \\
\hline & & 199903 & & $43.5 \cup$ \\
\hline
\end{tabular}

Trichloroethylene in $\mathrm{BGO} 33 \mathrm{C}$

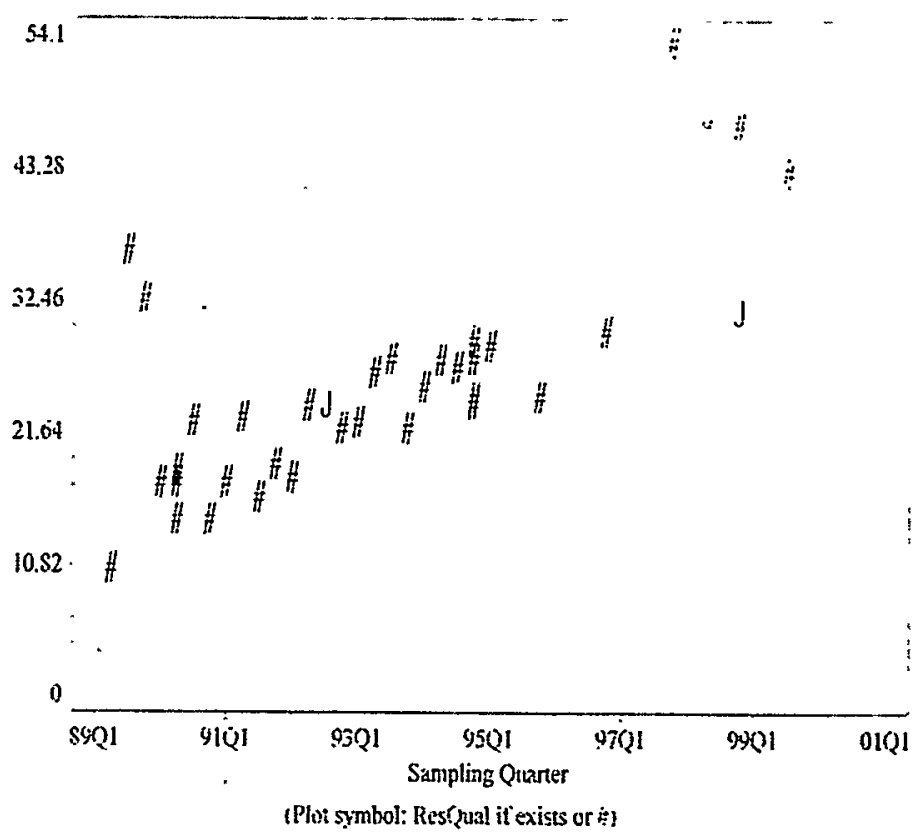


Gross alpha in BGO 33C

Data Retrieved by AWSA v1 from GIMS on 09NOV99 at 15:50

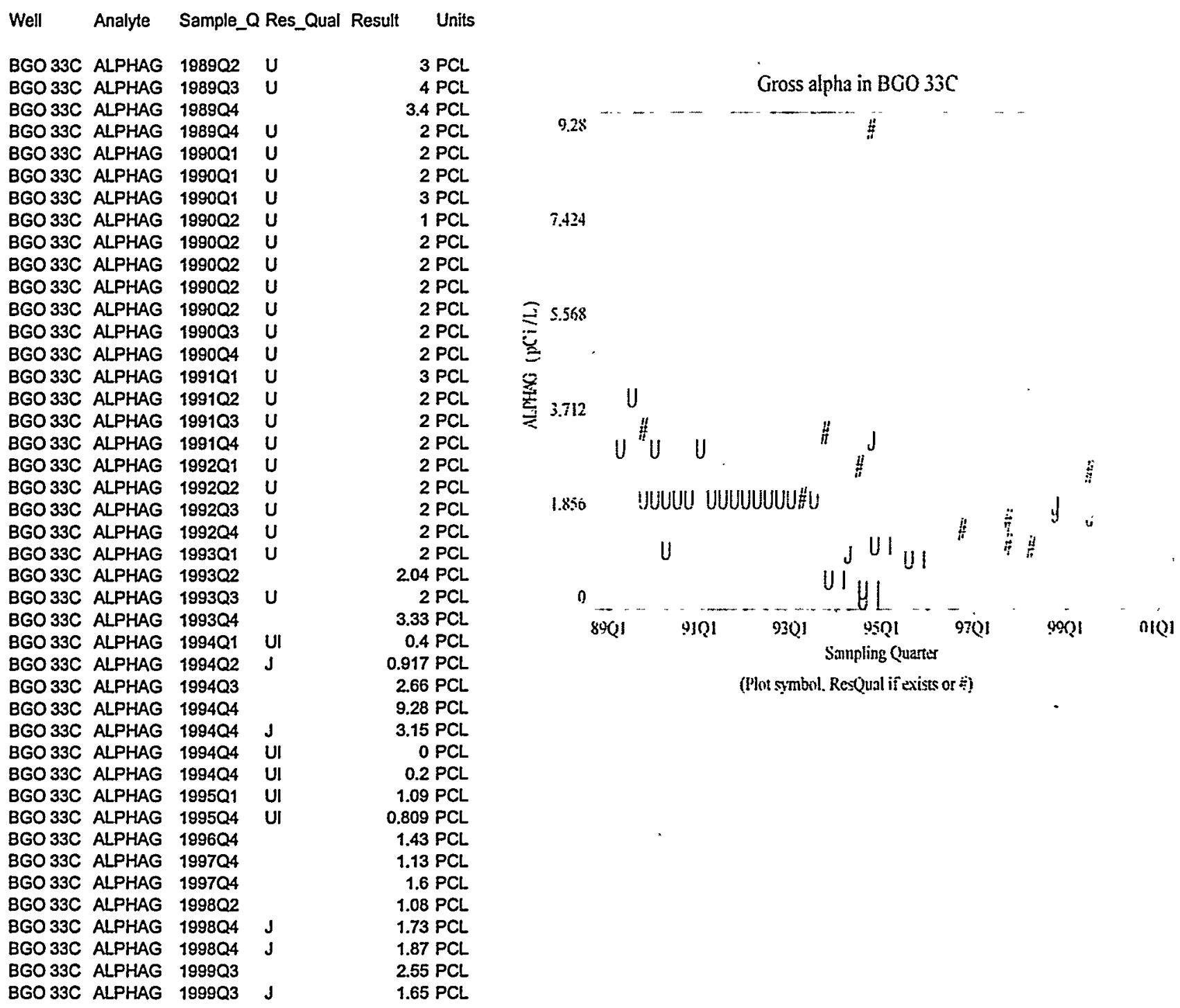


Tritium in BGO 33C

Data Retrieved by AWSA v1 from GIMS on 09NOV99 at 15:50

\begin{tabular}{|c|c|c|c|c|}
\hline Well & Analyte & Sample_Q Res_Qual & Result & Units \\
\hline BGO $33 C$ & TRITIU & 1989Q2 & 4400 & PCML \\
\hline BGO $33 \mathrm{C}$ & TRITIU & $1989 Q 2$ & 4400 & PCML \\
\hline BGO 33C & TRITIU & 198902 & 5255.1 & PCML \\
\hline BGO $33 \mathrm{C}$ & TRITIU & $1989 Q 3$ & 5000 & PCML \\
\hline GO $33 \mathrm{C}$ & TRITIU & $1989 Q 4$ & 5400 & $\mathrm{PCl}$ \\
\hline BGO 33C & TRITIU & $1989 Q 4$ & 6100 & $\mathrm{PCl}$ \\
\hline BGO $33 \mathrm{C}$ & TRITIU & 1990Q1 & 570 & \\
\hline BGO $33 \mathrm{C}$ & TRITIU & $1990 Q 1$ & 580 & \\
\hline BGO $33 \mathrm{C}$ & TRITIU & $1990 Q 1$ & 7160 & $\mathrm{PCN}$ \\
\hline BGO $33 \mathrm{C}$ & TRITIU & $1890 Q 2$ & 5200 & $\mathrm{PCl}$ \\
\hline BGO $33 C$ & TRITIU & $1990 Q 2$ & 5400 & $\mathrm{PCl}$ \\
\hline $\mathrm{BGO} 33 \mathrm{C}$ & TRITIU & $1990 \mathrm{Q} 2$ & 5900 & $\mathrm{PCN}$ \\
\hline BGO $33 \mathrm{C}$ & TRITIU & $1990 Q 2$ & 7190 & $\mathrm{PCM}$ \\
\hline$B G O 33 C$ & TRITIU & 1990Q2 & 7280 & $\mathrm{PCM}$ \\
\hline BGO 33C & TRITIU & 1990Q3 & 5400 & \\
\hline BGO 33C & TRITIU & $1890 Q 4$ & 5100 & \\
\hline BGO 33C & TRITIU & 1991Q1 & 5400 & \\
\hline BGO $33 \mathrm{C}$ & TRITIU & 1991Q2 & 6360 & \\
\hline $\mathrm{BGO} 33 \mathrm{C}$ & TRITIU & 1991Q3 & 6270 & $\mathrm{PCl}$ \\
\hline BGO 33C & TRITIU & 1991Q4 & 6840 & $\mathrm{PCl}$ \\
\hline $\mathrm{BGO} 33 \mathrm{C}$ & TRITIU & $1992 Q 1$ & 7570 & $\mathrm{PCN}$ \\
\hline BGO 33C & TRITIU & 199202 & 8020 & \\
\hline BGO 33C & TRITIU & 1992Q3 & 7400 & \\
\hline BGO 33C & TRITIU & $1992 Q 4$ & 6890 & $\mathrm{PC}$ \\
\hline $\mathrm{BGO} 33 \mathrm{C}$ & TRITIU & 1993Q1 & 6790 & PCML \\
\hline BGO 33C & TRITIU & 199302 & 7220 & PCML \\
\hline BGO 33C & TRITIU & 199303 & 8170 & PCML \\
\hline BGO $33 \mathrm{C}$ & TRITIU & $1993 Q 4$ & 8010 & PCML \\
\hline BGO $33 \mathrm{C}$ & TRITIU & $1994 Q 1$ & 8200 & $\mathrm{PC}$ \\
\hline BGO 33C & TRITIU & 199402 & 8780 & \\
\hline BGO $33 \mathrm{C}$ & TRITIU & $1994 Q 3$ & 10100 & $\mathrm{PCA}$ \\
\hline BGO $33 \mathrm{C}$ & TRITIU & $1994 Q 4$ & 10100 & PCML \\
\hline BGO 33C & TRITIU & $1994 Q 4$ & 10200 & PCML \\
\hline BGO $33 \mathrm{C}$ & TRITIU & $1994 Q 4$ & 10200 & $\mathrm{PCM}$ \\
\hline BGO 33C & TRITIU & $1994 Q 4$ & 11200 & $\mathrm{PCl}$ \\
\hline BGO 33C & TRITIU & $1994 Q 4$ & 11200 & $\mathrm{PCN}$ \\
\hline BGO $33 \mathrm{C}$ & TRITIU & 199501 & 10000 & $\mathrm{PCN}$ \\
\hline BGO 33C & TRITIU & $1995 Q 4$ & 11500 & PCML \\
\hline $8 G O 33 C$ & TRITIU & $1996 Q 4$ & 11400 & PCML \\
\hline BGO 33C & TRITIU & 1997Q4 & 12000 & $\mathrm{PCM}$ \\
\hline BGO 33C & TRITIU & 1997Q4 & 12100 & \\
\hline BGO 33C & TRITIU & 1998Q2 & 15400 & $\mathrm{PCN}$ \\
\hline $\mathrm{BGO} 33 \mathrm{C}$ & TRITIU & $1998 Q 4$ & 12800 & $\mathrm{PCN}$ \\
\hline BGO $33 \mathrm{C}$ & TRITIU & 1998Q4 & 14770 & PCML \\
\hline BGO 33C & TRITIU & $1999 Q 3$ & 13900 & $\mathrm{PCM}$ \\
\hline GO 33C & TRITIU & 1999Q3 & 14500 & \\
\hline
\end{tabular}

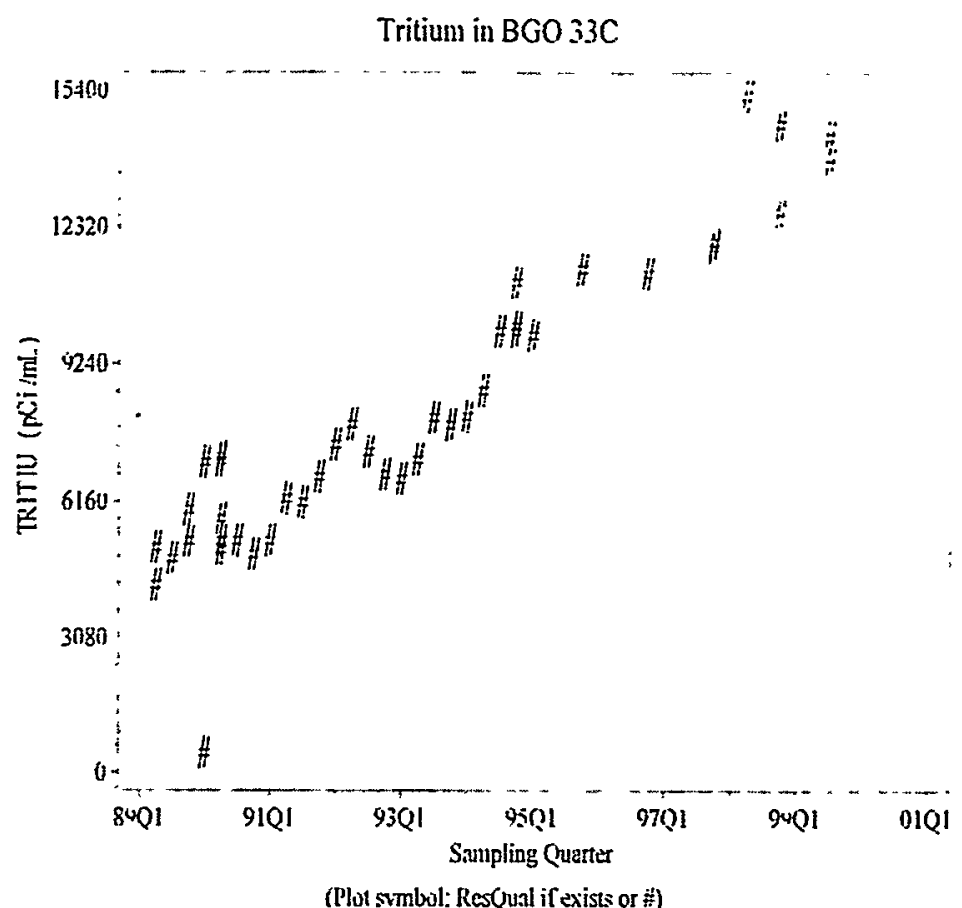

(Plut symbul: Res(ual if exists or :) 
1,1,1-Trichloroethane in BGO $33 \mathrm{C}$

Data Retrieved by AWSA v1 from GIMS on 09NOV99 at 15:50

Well Analyte Sample_QRes_Qual Result Units

BGO 33C 111TCE $1989 Q 2 U$ U $\quad 5$ UGL

BGO33C 111TCE 1989Q3 U 5 UGL

BGO 33C 111TCE 1989Q4 U

BGO 33C 111TCE 1990Q1 $U$ 1 UGL

BGO 33C 111TCE $199002 U$ U 1 UGL

BGO 33C 111TCE $199002 U$ Q $U$ UGL

BGO 33C 111TCE $199002 U$ U 5 UGL

BGO 33C 111TCE 199002 U 5 UGL

BGO 33C 111TCE $199003 \quad U$ U 5 UGL

BGO 33C 111TCE 1990Q4 U 5 UGL

BGO 33C 111TCE 1991Q1 U 5 UGL

BGO 33C 111TCE 1991Q2 U 1 UGL

BGO 33C 111TCE $199103 \mathrm{U}$ 1 UGL

BGO 33C 111TCE 1991Q4 U 1 UGL

BGO 33C 111TCE $1992 Q 1 U$ 1 UGL

BGO33C 111TCE

BGO 33C 111TCE

BGO 33C 111TCE

BGO 33C 111TCE

BGO 33C 111TCE

BGO 33C 111TCE

BGO 33C 111TCE

BGO 33C 111TCE

BGO 33C 111TCE

BGO 33C 111TCE

BGO 33C 111TCE

BGO 33C 111TCE

BGO 33C 111TCE

BGO 33C 111TCE

BGO 33C 111TCE

BGO 33C 111TCE

BGO 33C 111TCE

BGO 33C 111TCE

BGO 33C 111TCE

BGO 33C 111TCE

BGO 33C 111TCE

BGO 33C 111TCE
$1992 Q 2 U$

$1992 Q 3$ UJ

$1992 Q 4 U$

1993Q1 U

$199302 U$

1993Q3 U

$199304 \mathrm{U}$

$199401 U$

$199402 U$

$1994 Q 4 U$

1994Q4 U

$199404 \mathrm{U}$

1994Q4 U

1995Q1 U

1995Q4 U

1996Q4 U

$199704 U$

199802 UJ

$1998 Q 4 \mathrm{JU}$

$1998 Q 4 U$

199903
$1994 Q 3 U$

1 UGL

1 UGL

1 UGL

1 UGL

1 UGL

1 UGL

1 UGL

1 UGL

5 UGL

1 UGL

1 UGL

5 UGL

5 UGL

1.67 UGL

2 UGL

2 UGL

1 UGL

1 UGL

5 UGL

1 UGL

1 UGL
1.1,1-Trichloroethane in $\mathrm{BGO} 33 \mathrm{C}$

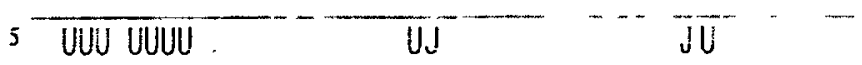

4

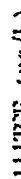

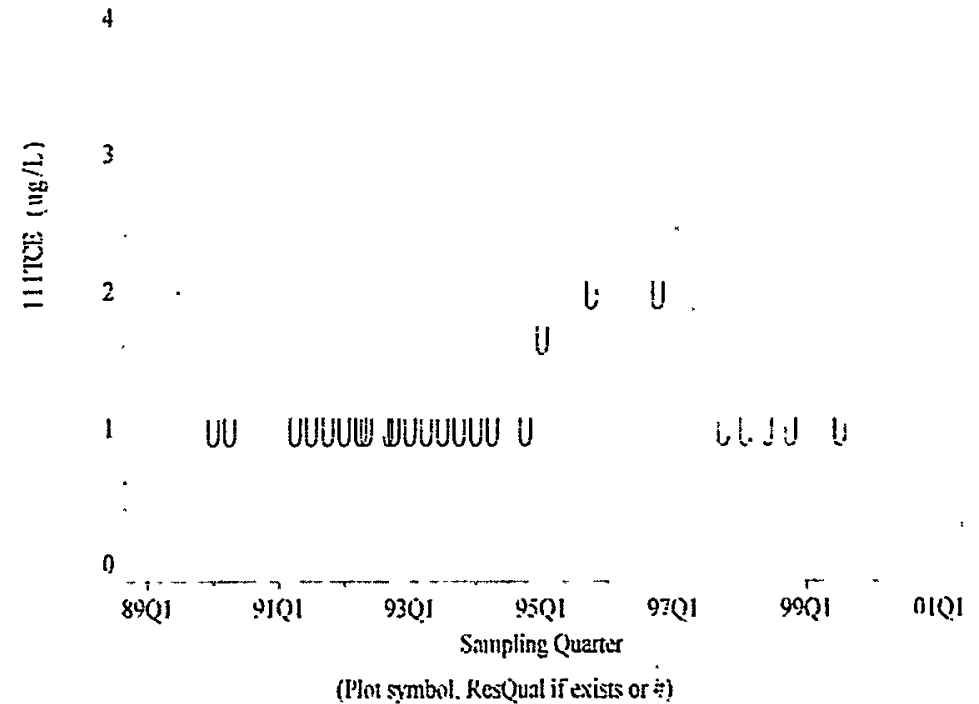

(IJot sqmbol. Kes(ual if exists or ti) 
1,1-Dichloroethylene in BGO $33 \mathrm{C}$

Data Retrieved by AWSA v1 from GIMS on 09NOV99 at 15:50
Sample_Q Res_Qual Result Units

$1989 Q 2 U$

1989Q3 U

$198904 U$

$199001 \mathrm{U}$

199002

$199002 \mathrm{U}$

$199002 U$

$199002 \mathrm{U}$

$199003 \mathrm{U}$

$1990 \mathrm{Q} 4 \mathrm{~J}$

$199101 \mathrm{U}$

$199102 \mathrm{U}$

$199103 U$

$199124 U$

1992Q1 U

$1992 Q 2 U$

1992Q3 UJ

1992Q4 U

199301 U

$199302 \mathrm{U}$

1993Q3 U

$1993 Q 4 U$

1994Q1 U

$199402 U$

1994Q3 U

1994Q4 U

$1994 Q 4 U$

$1994 Q 4 U$

1994Q4 U

1995Q1 U

1995Q4 U

1996Q4 U

1997Q4

$199802 \mathrm{~J}$

1998Q4

1998Q4 JU

1999Q3 J
5 UGL

5 UGL

5 UGL

1 UGL

1 UGL

1 UGL

5 UGL

5 UGL

5 UGL

3 UGL

5 UGL

1 UGL

1 UGL

1 UGL

1 UGL

1 UGL

1 UGL

1 UGL

1 UGL

1 UGL

1 UGL

1 UGL

1 UGL

1 UGL

5 UGL

1 UGL

1 UGL

5 UGL

5 UGL

1.67 UGL

2 UGL

2 UGL

1.27 UGL

0.758 UGL

1.11 UGL

5 UGL

0.935 UGL

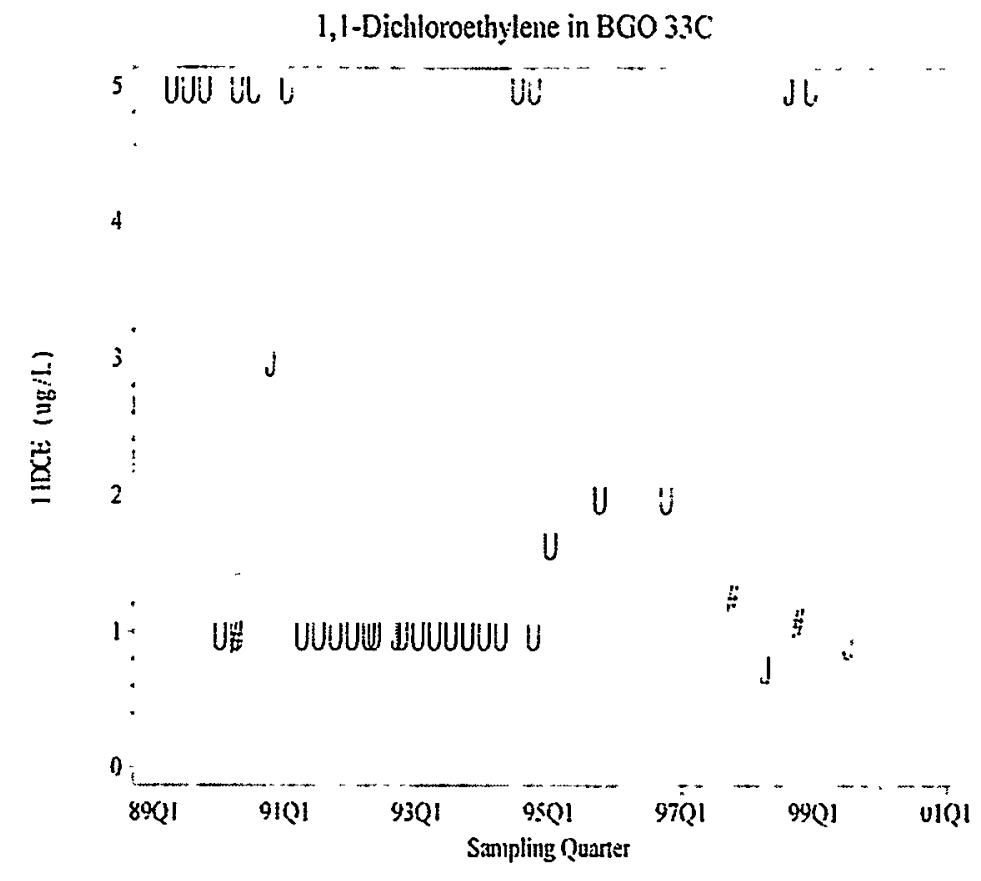

(Plot ssmbrol: Res(tual if exists of 
1.1-Dichloroethane in BGO $33 \mathrm{C}$

Data Retrieved by AWSA v1 from GIMS on 09NOV99 at 15:50

Well Analyte Sample_QRes_Qual Result Units

BGO 33C 11DCLE $1989 Q 2 U$ U 5 UGL

BGO 33C 11DCLE $1989 Q 3$ U 5 UGL

BGO 33C 11DCLE 198904 U 5 UGL

BGO 33C 11DCLE 1990Q1 U 1 UGL

BGO 33C 11DCLE 1990Q2 U 1 UGL

BGO 33C 11DCLE $199002 \mathrm{U}$ I UGL

BGO 33C 11DCLE $199002 \quad U$

BGO 33C 11DCLE 1990Q2 U 5 UGL

BGO 33C 11DCLE 1990Q3 U 5 UGL

BGO 33C 11DCLE 1990Q4 U 5 UGL

BGO 33C 11DCLE 1991Q1 U 5 UGL

BGO 33C 11DCLE $199102 \mathrm{U} \quad 1 \mathrm{UGL}$

BGO 33C 11DCLE 1991Q3 U 1 UGL

BGO 33C 11DCLE 1991Q4 U $1 \mathrm{UGL}$

BGO 33C 11DCLE 1992Q1 U 1 UGL

BGO 33C 11DCLE 199202 U

BGO 33C 11DCLE $1992 Q 3$ UJ

BGO 33C 11DCLE 1992Q4 U

BGO 33C 11DCLE 1993Q1 U

BGO 33C 11DCLE $199302 U$

BGO 33C 11DCLE 1993Q3 U

BGO 33C 11DCLE 1993Q4 U

BGO 33C 11DCLE 1994Q1 U

BGO 33C 11DCLE 1994Q2 U

BGO 33C 11DCLE 1994Q3 U

BGO 33C 11DCLE 1994Q4

BGO 33C 11DCLE 1994Q4 U

BGO 33C 11DCLE 1994Q4 U

BGO 33C 11DCLE 1994Q4 U

BGO 33C 11DCLE 1995Q1 U

BGO 33C 11DCLE 1995Q4 U

BGO 33C 11DCLE 1996Q4 U

BGO 33C 11DCLE 1997Q4

BGO 33C 11DCLE 199802

BGO 33C 11DCLE 1998Q4

BGO 33C 11DCLE 1998Q

BGO 33C 11DCLE 1999Q3
1 UGL

1 UGL

1 UGL

1 UGL

1 UGL

1 UGL

1 UGL

1 UGL

1 UGL

5 UGL

1 UGL

1 UGL

5 UGL

5 UGL

1.67 UGL

2 UGL

2 UGL

0.968 UGL

0.923 UGL

1.02 UGL

5 UGL

0.847 UGL
1,1-Dichloroethane in BGO 33C

U:

JU

4

$=3$

5 UUU UUUU

U U

U

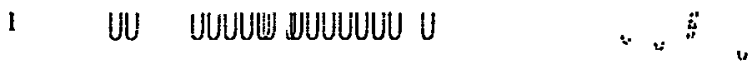

(i) -

8901 9101 9301 $\begin{gathered}9501 \\ \text { Sampling Quarter }\end{gathered}$

(Plut şmbol: Res(ual if exists or 
Barium, total recoverable in BGO $33 \mathrm{C}$

Data Retrieved by AWSA v1 from GIMS on 09NOV99 at 15:50

Well Analyte Sample_Q Res_Qual Result Units

BGO 33C BATOT 1992Q4

BGO 33C BATOT 1993Q1

BGO 33C BATOT 199302

BGO 33C BATOT 1993Q3

BGO 33C BATOT 199303

BGO 33C BATOT 1993Q4

BGO 33C BATOT 199401

BGO 33C BATOT 199402

BGO 33C BATOT 199403

BGO 33C BATOT 199404

BGO 33C BATOT 199404

BGO 33C BATOT 1994Q4

BGO 33C BATOT $1994 Q 4$

BGO 33C BATOT 1994Q4

BGO 33C BATOT 1995Q1

BGO 33C BATOT 1995Q4

BGO 33C BATOT 1996Q

BGO 33C BATOT 1997Q4

BGO 33C BATOT 199802

BGO 33C BATOT 1998Q4

BGO 33C BATOT 1998Q4

BGO 33C BATOT $1999 Q 3$
12.8 UGL 12.2 UGL

12.1 UGL

12.1 UGL

12.5 UGL

10.9 UGL

10.9 UGL

10.8 UGL

10.7 UGL

10.6 UGL

10.7 UGL

10.8 UGL

10.8 UGL

10.8 UGL

10.7 UGL

10.9 UGL

11.3 UGL

13.1 UGL

11.2 UGL

10.6 UGL

10.9 UGL

11.5 UGL

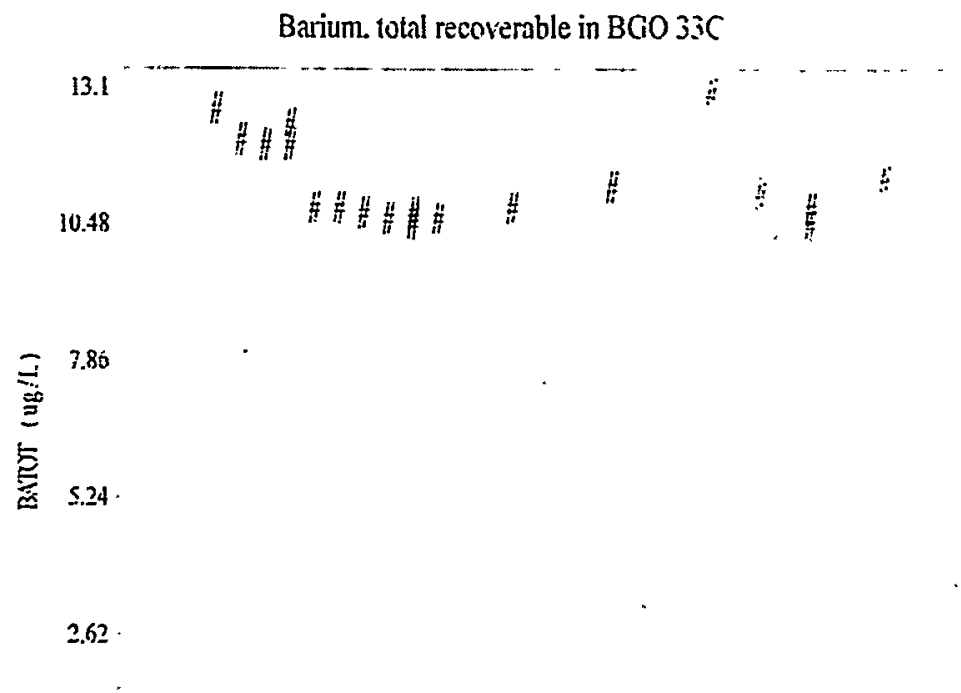

0

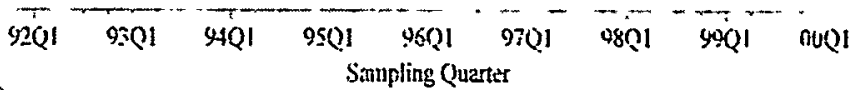

(PInt symbonl. Res(unal if exists or ä) 
Nonvolatile beta in BGO $33^{\circ}$

Data Retrieved by AWSA v1 from GIMS on 09NOV99 at 15:50

\begin{tabular}{|c|c|c|c|c|}
\hline Well & Analyte & Sample_o & Res_Qual & Result \\
\hline BGO $33 \mathrm{C}$ & BETAG & $1989 Q 2$ & $u$ & $5 \mathrm{PCL}$ \\
\hline BGO $33 \mathrm{C}$ & BETAG & $1989 Q 3$ & u & $4 \mathrm{PCL}$ \\
\hline BGO $33 C$ & BETAG & 198904 & & 5.1 PCL \\
\hline BGO $33 \mathrm{C}$ & BETAG & 198904 & & $5.8 \mathrm{PCL}$ \\
\hline BGO 33C & BETAG & 1990Q1 & & $2.7 \mathrm{PCL}$ \\
\hline BGO $33 C$ & BETAG & 199001 & $U$ & $2 \mathrm{PCL}$ \\
\hline BGO $33 \mathrm{C}$ & BETAG & 1990Q1 & $\mathrm{U}$ & $2 \mathrm{PCL}$ \\
\hline BGo $33 \mathrm{C}$ & BETAG & $1990 Q 2$ & & $1.6 \mathrm{PCL}$ \\
\hline BGO $33 C$ & BETAG & 199002 & & 3.4 PCL \\
\hline BGO $33 \mathrm{C}$ & BETAG & 199002 & $U$ & $2 \mathrm{PCL}$ \\
\hline BGO 33C & BETAG & $1990 Q 2$ & U & $4 \mathrm{PCL}$ \\
\hline BGO $33 \mathrm{C}$ & BETAG & $1990 Q 2$ & U & $4 \mathrm{PCL}$ \\
\hline BGO $33 \mathrm{C}$ & BETAG & $1990 Q 3$ & U & $5 \mathrm{PCL}$ \\
\hline BGO $33 C$ & BETAG & 199004 & $U$ & $4 \mathrm{PCL}$ \\
\hline BGO $33 \mathrm{C}$ & BETAG & 1991Q1 & U & $4 \mathrm{PCL}$ \\
\hline BGO 33C & BETAG & 199102 & $u$ & $2 \mathrm{PCL}$ \\
\hline BGO $33 \mathrm{C}$ & BETAG & 1991Q3 & $U$ & $2 \mathrm{PCL}$ \\
\hline BGO 33C & BETAG & $1991 Q 4$ & $u$ & $2 \mathrm{PCL}$ \\
\hline BGO $33 C$ & BETAG & 1992Q1 & $u$ & $2 \mathrm{PCL}$ \\
\hline BGO 33C & BETAG & 199202 & $u$ & $2 \mathrm{PCL}$ \\
\hline BGO $33 \mathrm{C}$ & BETAG & 199203 & $u$ & $2 \mathrm{PCL}$ \\
\hline BGO 33C & BETAG & $1992 Q 4$ & $u$ & $2 \mathrm{PCL}$ \\
\hline BGO 33C & BETAG & 199301 & $u$ & $2 \mathrm{PCL}$ \\
\hline BGO $33 \mathrm{C}$ & BETAG & 199302 & & 5.12 PCL \\
\hline BGO $33 \mathrm{C}$ & BETAG & 199303 & $U$ & $2 \mathrm{PCL}$ \\
\hline BGO $33 \mathrm{C}$ & BETAG & $1993 Q 4$ & & $3.28 \mathrm{PCL}$ \\
\hline BGO $33 C$ & BETAG & 1994Q1 & UI & $0.6 \mathrm{PCL}$ \\
\hline BGO $33 C$ & BETAG & 199402 & $\mathrm{~J}$ & $1.04 \mathrm{PCL}$ \\
\hline BGO $33 C$ & BETAG & $1994 Q 3$ & & $2.57 \mathrm{PCL}$ \\
\hline BGO $33 C$ & BETAG & $1994 Q 4$ & & $5.73 \mathrm{PCL}$ \\
\hline BGO $33 \mathrm{C}$ & BETAG & 1994Q4 & & 8.85 PCL \\
\hline BGO $33 \mathrm{C}$ & BETAG & $1994 Q 4$ & اU & $0.5 \mathrm{PCL}$ \\
\hline BGO 33C & BETAG & 1994Q4 & Iا & $1.4 \mathrm{PCL}$ \\
\hline BGO $33 C$ & BETAG & 199501 & U! & $1.45 \mathrm{PCL}$ \\
\hline BGO $33 C$ & BETAG & 199504 & & $2.53 \mathrm{PCL}$ \\
\hline BGO $33 \mathrm{C}$ & BETAG & 1996Q4 & & $2.75 \mathrm{PCL}$ \\
\hline BGO $33 \mathrm{C}$ & BETAG & $1997 Q 4$ & & $1.51 \mathrm{PCL}$ \\
\hline BGO $33 \mathrm{C}$ & BETAG & $1997 Q 4$ & Ul & $1.17 \mathrm{PCL}$ \\
\hline BGO $33 \mathrm{C}$ & BETAG & 199802 & UI & $0.718 \mathrm{PCL}$ \\
\hline BGO $33 \mathrm{C}$ & BETAG & 1998Q4 & J & $2.31 \mathrm{PCL}$ \\
\hline BGO $33 \mathrm{C}$ & BETAG & $1998 Q 4$ & $u$ & 1.73 PCL \\
\hline BGO $33 \mathrm{C}$ & BETAG & $1999 Q 3$ & $J$ & $1.56 \mathrm{PCL}$ \\
\hline BGO $33 \mathrm{C}$ & BETAG & 1999Q3 & $J$ & 1.97 PCL \\
\hline
\end{tabular}

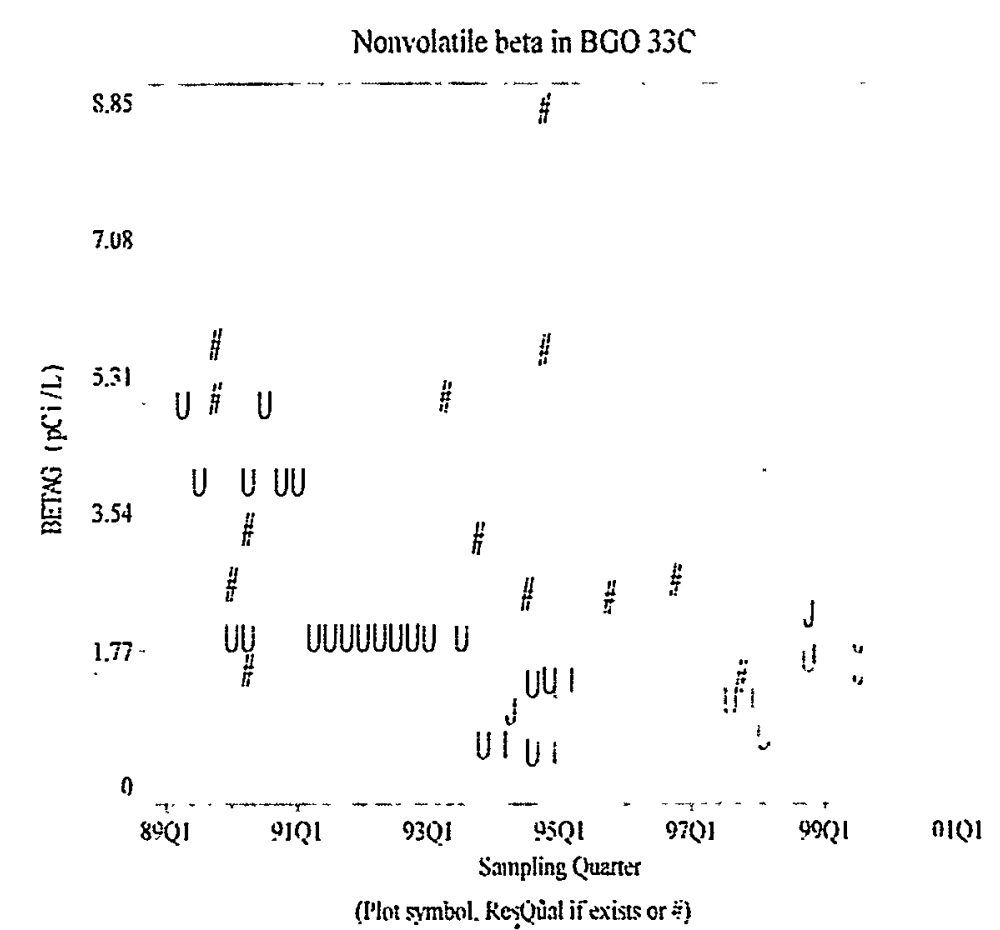


Cobalt, total recoverable in BGO $33 \mathrm{C}$

Data Retrieved by AWSA v1 from GIMS on 09NOV99 at 15:50

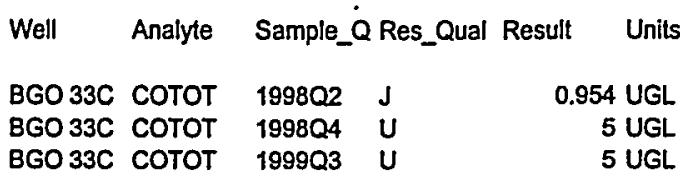

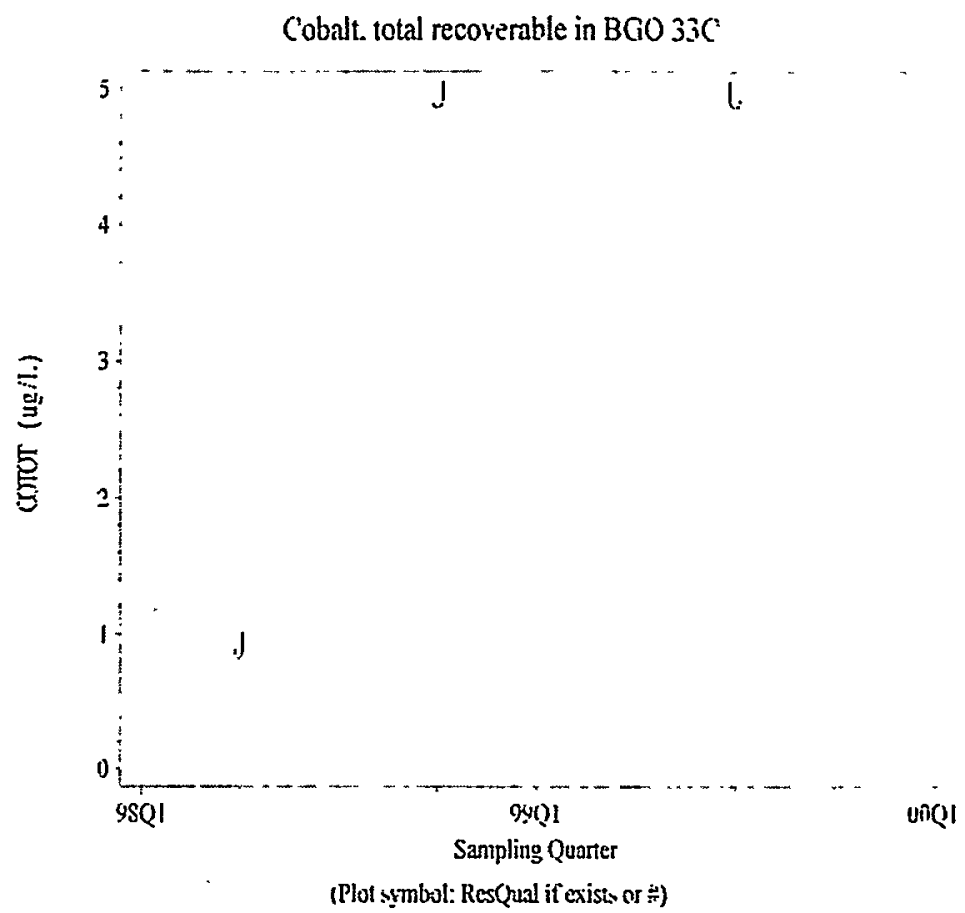


Copper, total recoverable in BGO $33 \mathrm{C}$

Data Retrieved by AWSA v1 from GIMS on 09NOV99 at 15:50

\begin{tabular}{|c|c|c|c|c|c|}
\hline Well & Analyte & Sample_Q & Res_Qual & Result & Units \\
\hline BGO 33C & CUTOT & 199204 & $U$ & & 4 UGL \\
\hline BGO $33 \mathrm{C}$ & CUTOT & 1993Q1 & U & & 4 UGL \\
\hline $3 G 033 \mathrm{C}$ & CUTOT & $1993 Q 2$ & u & & 4 UGL \\
\hline $\mathrm{BGO} 33 \mathrm{C}$ & CUTOT & 1993Q3 & $u$ & & 4 UGL \\
\hline $3 G 033 \mathrm{C}$ & CUTOT & $1993 Q 3$ & U & & 4 UGL \\
\hline BGO $33 C$ & CUTOT & $1993 Q 4$ & U & & 4 UGL \\
\hline BGO $33 \mathrm{C}$ & CUTOT & 1994Q1 & $u$ & & 4 UGL \\
\hline BGO 33C & CUTOT & $1994 Q 2$ & U & & 4 UGL \\
\hline $\mathrm{BGO} 33 \mathrm{C}$ & CUTOT & 1994Q3 & $u$ & & 4 UGL \\
\hline$B G O 33 C$ & CUTOT & $1994 Q 4$ & U & & 4 UGL \\
\hline BGO $33 \mathrm{C}$ & CUTOT & $1994 Q 4$ & U & & 4 UGL \\
\hline $\mathrm{BGO} 33 \mathrm{C}$ & CUTOT & 1994Q4 & U & & 4 UGL \\
\hline $\mathrm{BGO} 33 \mathrm{C}$ & CUTOT & $1994 Q 4$ & U & & 4 UGL \\
\hline $\mathrm{BGO} 33 \mathrm{C}$ & CUTOT & 1994Q4 & $U$ & & 4 UGL \\
\hline BGO 33C & CUTOT & $1995 Q 1$ & $U$ & & 6.67 UGL \\
\hline $\mathrm{BGO} 33 \mathrm{C}$ & CUTOT & $1996 Q 4$ & & & B.21 UGL \\
\hline BGO 33C & СUTOT & $1997 Q 4$ & $U$ & & 5 UGL \\
\hline BGO 33C & CUTOT & 199802 & & & 13.8 UGL \\
\hline $8 G 033 \mathrm{C}$ & CUTOT & 1998Q4 & $J$ & & $1.8 \mathrm{UGL}$ \\
\hline BGO $33 \mathrm{C}$ & CUTOT & $1998 Q 4$ & $\mathbf{J}$ & & 4.34 UGL \\
\hline BGO 33C & CUTOT & 1999Q3 & $J$ & & 3.6 UGL \\
\hline
\end{tabular}

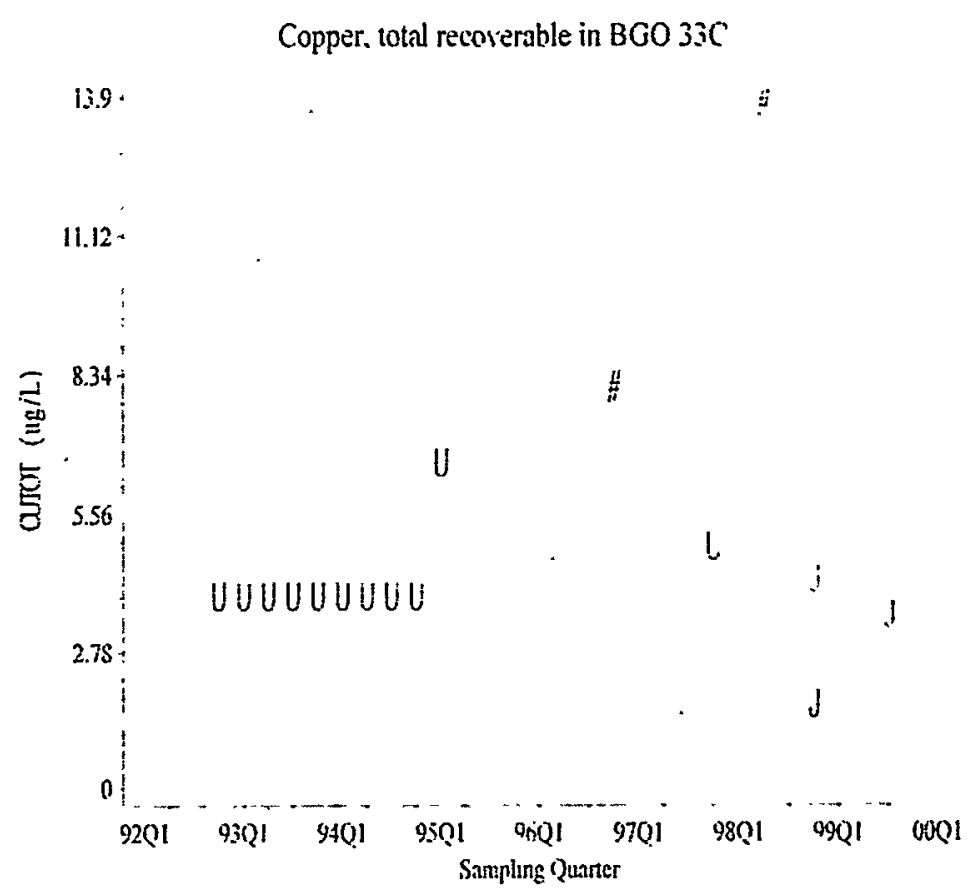

(Plot syinbol: Res(qual if exists or 
Cyanide in $\mathrm{BGO} 33 \mathrm{C}$

Data Retrieved by AWSA v1 from GIMS on 09NOV99 at 15:50
Sample_Q Res_Qual Result Units 1991Q1 U 5 UGL $199102 \mathrm{U} \quad 5$ UGL $199103 U$ U 5 UGL 199104 UJ 5 UGL $1992 \mathrm{Q}$ UJ 5 UGL $1992 Q 2 U$ U UGL 199203 UJ 5 UGL 1992Q4 U 5 UGL $199301 U$ U UGL $199302 U \quad 10$ UGL 199303 UJ 5 UGL $199304 U$ U UGL $1994 Q 1 U$ U UGL 1994 2 U 5 UGL $199403 U$ U 5 UGL 1994 Q4 $U$ S UGL $199404 \mathrm{U} 5 \mathrm{UGL}$ $1994 Q 4 U$ U UGL $199404 U$ U 5 UGL $1994 Q 4 U$ U UGL 1995 Q1 UJ 8.33 UGL 199802 UJ 10 UGL $1998 Q 4 U$ U 10 UG $199903 U \quad 10 \mathrm{UGL}$

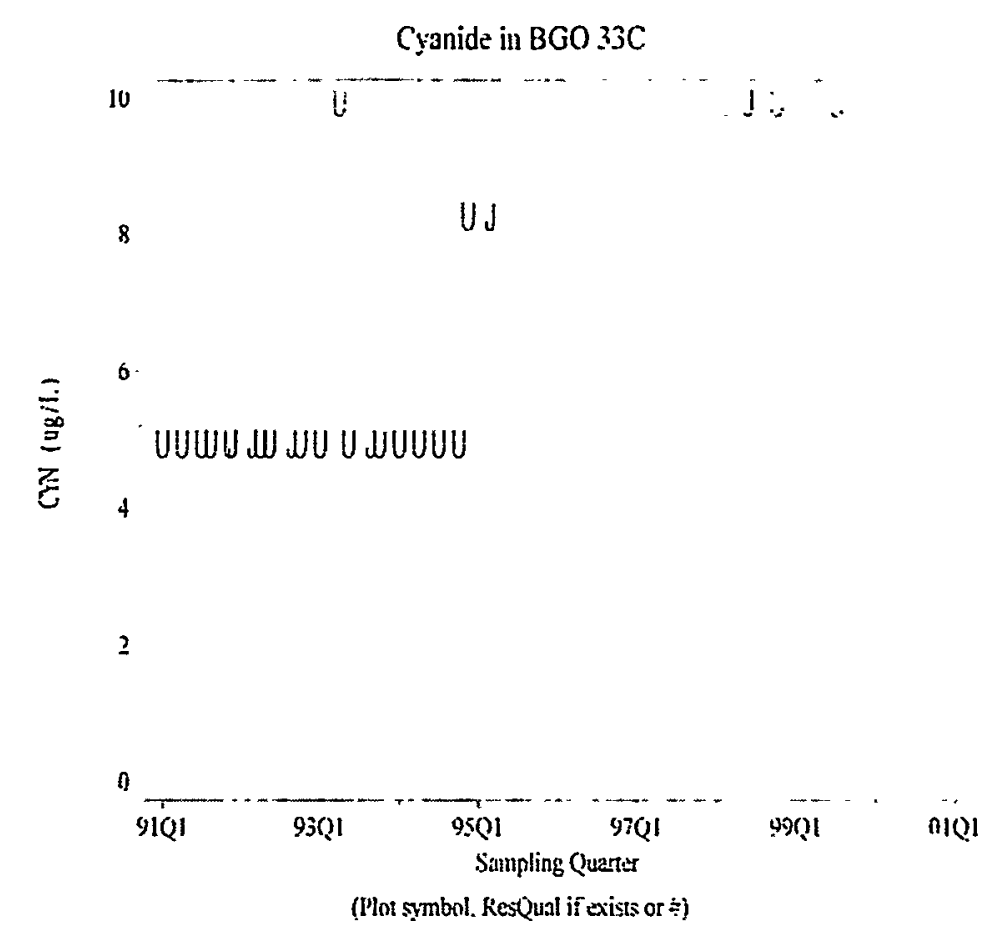


Fluoride in BGO $33 \mathrm{C}$

Data Retrieved by AWSA v1 from GIMS on 09NOV99 at 15:50

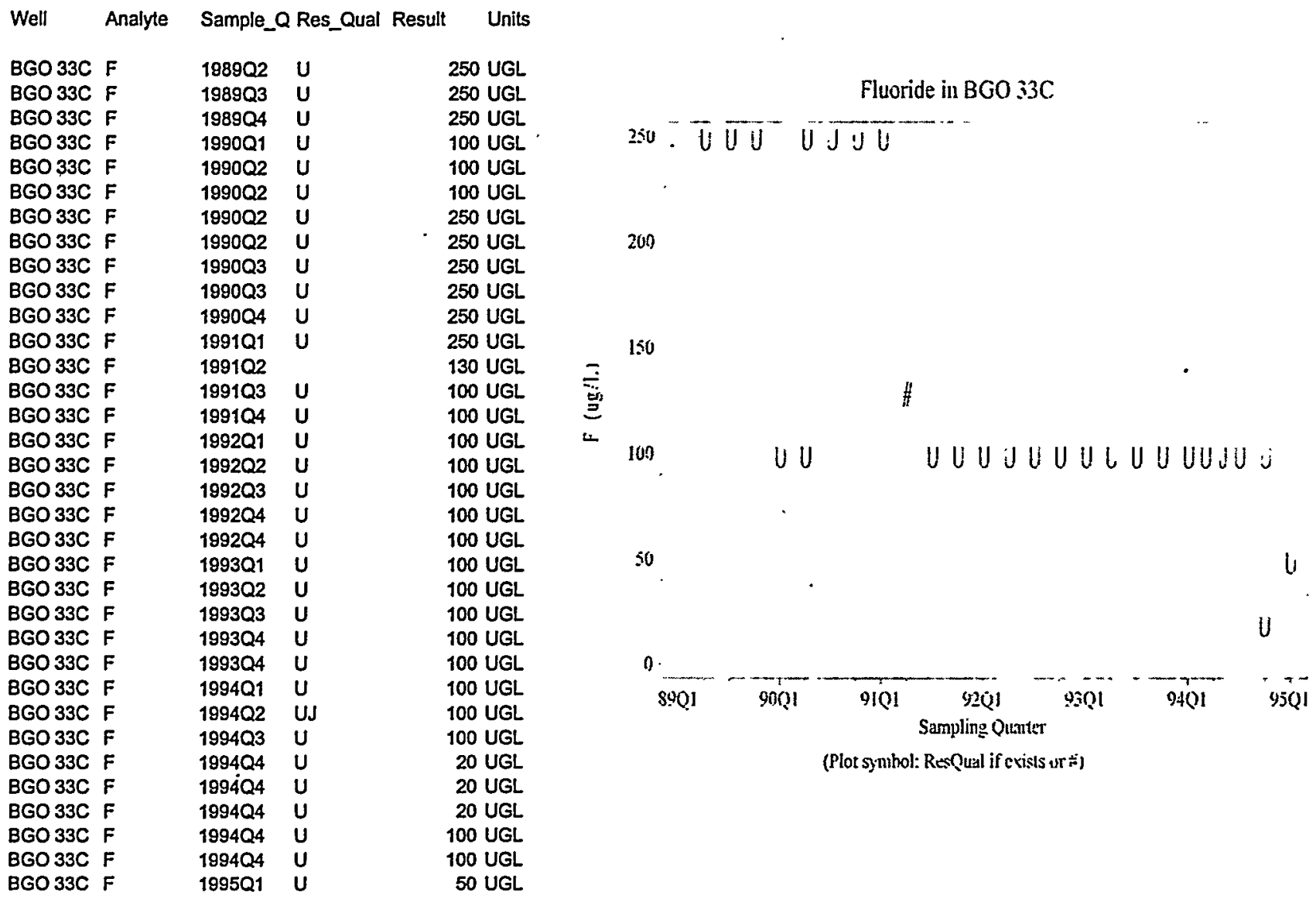


Mercury, total recoverable in BGO $33 \mathrm{C}$

Data Retrieved by AWSA v1 from GIMS on 09NOV99 at 15:50

\begin{tabular}{|c|c|c|c|c|}
\hline Well & Analyte & Sample_Q Res_Qual & Result & Units \\
\hline BGO 33C & HGTOT & $1992 Q 4$ & & 66 UGL \\
\hline BGO 33C & HGTOT & 1993Q1 & & 46 UGL \\
\hline BGO $33 \mathrm{C}$ & HGTOT & 199302 & & 68 UGL \\
\hline BGO $33 \mathrm{C}$ & HGTOT & $1993 Q 3$ & 2.2 & 21 UGL \\
\hline BGO 33C & HGTOT & $1993 Q 4$ & 2.3 & 37 UGL \\
\hline BGO 33C & HGTOT & 1994Q1 & 2.7 & 79 UGL \\
\hline $\mathrm{BGO} 33 \mathrm{C}$ & HGTOT & 1994Q2 & 1.2 & 26 UGL \\
\hline $\mathrm{BGO} 33 \mathrm{C}$ & HGTOT & $1994 Q 2$ & & 36 UGL \\
\hline BGO $33 \mathrm{C}$ & HGTOT & $1994 Q 3$ & & 3.4 UGL \\
\hline $\mathrm{BGO} 33 \mathrm{C}$ & HGTOT & $1994 Q 4$ & & 79 UGL \\
\hline BGO 33C & HGTOT & $1994 Q 4$ & & 35 UGL \\
\hline BGO 33C & HGTOT & $1994 Q 4$ & & 39 UGL \\
\hline BGO $33 \mathrm{C}$ & HGTOT & $1994 Q 4$ & 4.1 & 11 UGL \\
\hline $\mathrm{BGO} 33 \mathrm{C}$ & HGTOT & $1995 Q 1$ & & 97 UGL \\
\hline BGO $33 \mathrm{C}$ & HGTOT & 1995Q4 & & 43 UGL \\
\hline BGO 33C & HGTOT & $1996 Q 4$ & & 5.4 UGL \\
\hline BGO 33C & HGTOT & $1997 Q 4$ & & 67 UGL: \\
\hline BGO 33C & HGTOT & $1998 Q 2$ & & 42 UGL \\
\hline BGO $33 \mathrm{C}$ & HGTOT & 1998Q4 & & 5.1 UGL \\
\hline BGO 33C & HGTOT & $1998 Q 4$ & 5.7 & 76 UGL \\
\hline BGO 33C & HGTOT & 1999Q3 & & 6.5 UGL \\
\hline
\end{tabular}

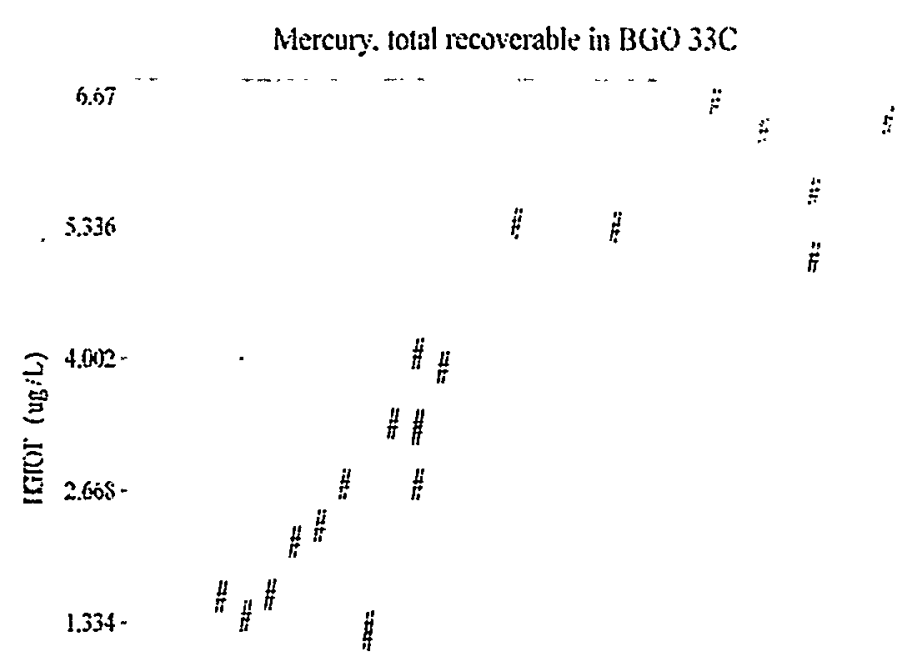

0 . 9201 9301 9401 95Q1 9001 9701 9501 9yol onQ1 (Plol symbol: Res?uad if exists of F) 
Nickel, total recoverable in BGO $33 \mathrm{C}$

Data Retrieved by AWSA v1 from GIMS on 09NOV99 at 15:50

\begin{tabular}{llllr} 
Well & Analyte & \multicolumn{2}{c}{ Sample_Q Res_Qual Result } & Units \\
& & & \\
BGO 33C NITOT & $1892 Q 4$ & $U$ & 4 UGL \\
BGO 33C NITOT & $1993 Q 1$ & $U$ & 4 UGL \\
BGO 33C NITOT & $1993 Q 2$ & & 5.02 UGL \\
BGO 33C NITOT & $1993 Q 3$ & $U$ & 4 UGL \\
BGO 33C NITOT & $1993 Q 3$ & $U$ & 4 UGL \\
BGO 33C NITOT & $1993 Q 4$ & $U$ & 4 UGL \\
BGO 33C NITOT & $1994 Q 1$ & $U$ & 4 UGL \\
BGO 33C NITOT & $1994 Q 2$ & $U$ & 4 UGL \\
BGO 33C NITOT & $1994 Q 3$ & $U$ & 4 UGL \\
BGO 33C NITOT & $1994 Q 4$ & $U$ & 4 UGL \\
BGO 33C NITOT & $1994 Q 4$ & $U$ & 4 UGL \\
BGO 33C NITOT & $1994 Q 4$ & $U$ & 4 UGL \\
BGO 33C NITOT & $1994 Q 4$ & $U$ & 4 UGL \\
BGO 33C NITOT & $1994 Q 4$ & $U$ & 4 UGL \\
BGO 33C NITOT & $1995 Q 1$ & $U$ & 6.67 UGL \\
BGO 33C NITOT & $1995 Q 4$ & $J$ & 0.987 UGL \\
BGO 33C NITOT & $1996 Q 4$ & $\mathrm{~J}$ & 1.23 UGL \\
BGO 33C NITOT & $1997 Q 4$ & $\mathrm{~J}$ & 2.9 UGL \\
BGO 33C NITOT & $1998 Q 2$ & $\mathrm{~J}$ & 3.28 UGL \\
BGO 33C NITOT & $1998 Q 4$. & $\mathrm{J}$ & 2.7 UGL \\
BGO 33C NITOT & $1998 Q 4$ & $\mathrm{JU}$ & 1.39 UGL \\
BGO 33C NITOT & $1999 Q 3$ & $\mathrm{~J}$ & 3.3 UGL
\end{tabular}

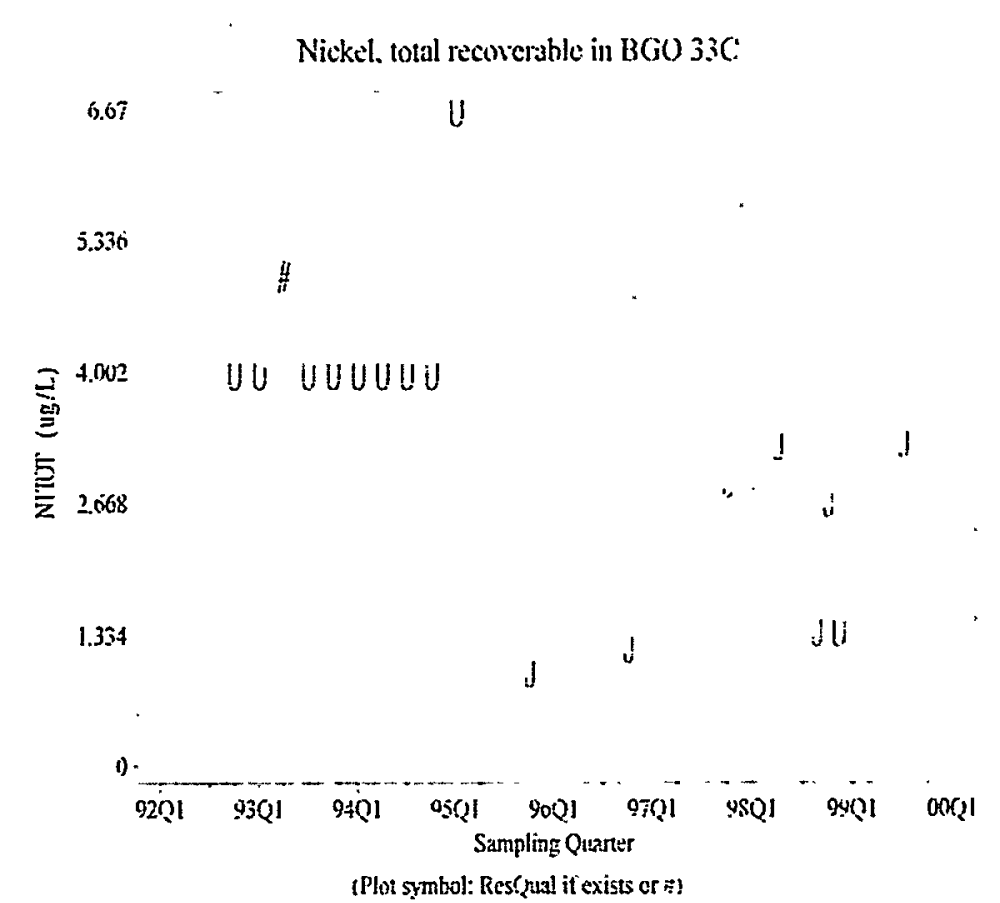


Nitrate-nitrite as nitrogen in $\mathrm{BGO} 33 \mathrm{C}$

Data Retrieved by AWSA v1 from GIMS on 09NOV99 at 15:50

Well Analyte Sample_Q Res_Qual Result Units

BGO 33C NO3NO2 1991Q4

BGO 33C NO3NO2 199104

BGO 33C NO3NO2 199104

BGO 33C NO3NO2 1991Q4

BGO 33C NO3NO2 1992Q1

BGO 33C NO3NO2 199202

BGO 33C NO3NO2 $1992 \mathrm{Q} 2$

BGO 33C NO3NO2 $1992 \mathrm{Q} 3$

BGO 33C NO3NO2 $1992 \mathrm{Q} 4$

BGO 33C NO3NO2 $1992 \mathrm{Q} 4$

BGO 33C NO3NO2 1993Q1

BGO 33C NO3NO2 199302

BGO 33C NO3NO2 $1993 \mathrm{Q} 3$

BGO 33C NO3NO2 1993Q4

BGO 33C NO3NO2 1993Q4

BGO 33C NO3NO2 $1994 Q 1$

BGO 33C NO3NO2 1894Q1

BGO 33C NO3NO2 199402

BGO 33C NO3NO2 1994Q4

BGO 33C NO3NO2 1994Q4

BGO 33C NO3NO2 1994Q4 J

BGO 33C NO3NO2 1995Q1
950 UGL 950 UGL 950 UGL 950 UGL 1060 UGL 1130 UGL 1140 UGL 1110 UGL 5300 UGL 5700 UGL 1170 UGL 1270 UGL 1110 UGL 1080 UGL 1080 UGL 930 UGL 940 UGL 990 UGL 1090 UGL 1140 UGL 1120 UGL 1110 UGL
Nitrate-nitrite as uitrogen in $B G O 33 C$

$$
\text { ii }
$$$$
\text { it }
$$

4560

23420

열

3

2280

1140

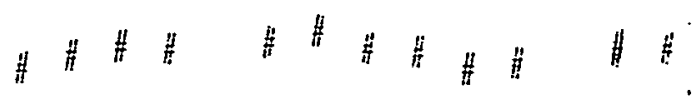

i)

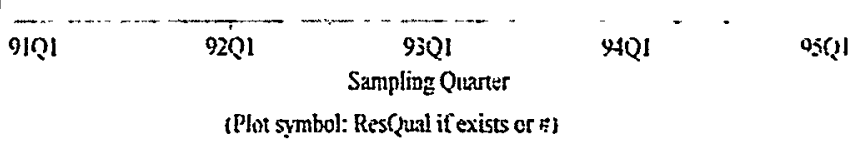


Selenium, total recoverable in BGO $33 \mathrm{C}$

Data Retrieved by AWSA v1 from GIMS on 09NOV99 at 15:50

Well Analyte Sample_QRes_Qual Result Units

BGO $33 \mathrm{C}$ SETOT

BGO 33C SETOT

BGO 33C SETOT

BGO 33C SETOT

BGO 33C SETOT

BGO $33 C$ SETOT

BGO 33C SETOT

BGO 33C SETOT

BGO 33C SETOT

BGO 33 C SETOT

BGO 33C SETOT

BGO 33C SETOT

BGO 33C SETOT

BGO 33C SETOT

BGO $33 C$ SETOT

BGO 33C SETOT

BGO 33C SETOT

BGO 33C SETOT

BGO 33C SETOT

BGO 33C SETOT

BGO 33C SETOT

BGO 33C SETOT

BGO 33C SETOT

$\begin{array}{ll}1992 Q 4 & U \\ 1993 Q 1 & U \\ 1993 Q 2 & U \\ 1993 Q 3 & U \\ 1993 Q 3 & U \\ 1993 Q 4 & U \\ 1994 Q 1 & U \\ 1994 Q 2 & U \\ 1994 Q 3 & U \\ 1994 Q 4 & U \\ 1994 Q 4 & U \\ 1994 Q 4 & U \\ 1994 Q 4 & U \\ 1994 Q 4 & U \\ 1995 Q 1 & U \\ 1995 Q 1 & U \\ 1995 Q 4 & U \\ 1996 Q 4 & J \\ 1997 Q 4 & J \\ 1998 Q 2 & J \\ 1998 Q 4 & J U \\ 1998 Q 4 & U \\ 1999 Q 3 & U\end{array}$

2 UGL

2 UGL

2 UGL

2 UGL

2 UGL

2 UGL

2 UGL

2 UGL

2 UGL

2 UGL

2 UGL

2 UGL

2 UGL

2 UGL

3.33 UGL

3.33 UGL

5 UGL

3.13 UGL

2.44 UGL

4.05 UGL

3.01 UGL

66 UGL

5 UGL
Selenium, total recoverable in $\mathrm{BGO} 330^{\circ}$

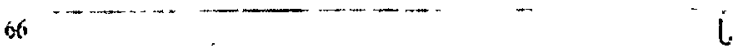

528

$\equiv 396$

宊

官

26.4

13.2

\begin{tabular}{|c|c|c|c|c|c|c|c|}
\hline D & uUuveus & $U^{U}$ & U & & & : & \\
\hline 9201 & 9301 & 4501 & $96 \mathrm{Q}$ & Y:ن̈1 & 9801 & 990 & well \\
\hline
\end{tabular}


trans-1,2-Dichloroethylene in BGO $33 \mathrm{C}$

Data Retrieved by AWSA v1 from GIMS on 09NOV99 at 15:50

\begin{tabular}{|c|c|c|c|c|}
\hline Well & Analyte & Sample_Q & Res_Qual & Result \\
\hline $\mathrm{BGO} 33 \mathrm{C}$ & T12DCE & 1989Q2 & U & 5 UGL \\
\hline BGO $33 \mathrm{C}$ & T120CE & $1989 Q 3$ & & 190 UGL \\
\hline BGO $33 \mathrm{C}$ & T12DCE & $1989 Q 4$ & $u$ & 5 UGL \\
\hline BGO $33 \mathrm{C}$ & T12DCE & 1990Q1 & $u$ & 1 UGL \\
\hline BGO $33 \mathrm{C}$ & T12DCE & 199002 & $U$ & 1 UGL \\
\hline BGO $33 \mathrm{C}$ & T12DCE & 199002 & U & 1 UE \\
\hline BGO $33 \mathrm{C}$ & T12DCE & 199002 & U & 5 UGL \\
\hline BGO 33C & T12DCE & 199002 & $u$ & 5 UGL \\
\hline BGO 33C & T12DCE & $1990 Q 3$ & & 190 UGL \\
\hline BGO $33 \mathrm{C}$ & T12DCE & $1990 Q 4$ & U & 5 UGL \\
\hline BGO $33 \mathrm{C}$ & T12DCE & 1991Q1 & & 130 UGL \\
\hline BGO 33C & T12DCE & $1991 Q 2$ & $u$ & 1 UGL \\
\hline BGO $33 \mathrm{C}$ & T12DCE & 1991Q3 & u & 1 UGL \\
\hline BGO $33 \mathrm{C}$ & T12DCE & 1991Q4 & U & 1 UGL \\
\hline BGO $33 \mathrm{C}$ & T12DCE & $1992 Q 1$ & U & 1 UGL \\
\hline BGO $33 \mathrm{C}$ & T12DCE & $1992 Q 2$ & $\mathbf{U}$ & 1 UGL \\
\hline BGO 33C & T12DCE & $1992 Q 3$ & UJ & 1 UGL \\
\hline BGO $33 \mathrm{C}$ & T120CE & $1992 Q 4$ & $\mathbf{U}$ & 1 UC \\
\hline $\mathrm{BGO} 33 \mathrm{C}$ & T12DCE & 1993Q1 & u & 1 UGL \\
\hline $\mathrm{BGO} 33 \mathrm{C}$ & T12DCE & 199302 & U & 1 UGL \\
\hline $\mathrm{BGO} 33 \mathrm{C}$ & T12DCE & $1993 Q 3$ & $u$ & 1 UGL \\
\hline BGO 33C & T12DCE & 199304 & U & 1 UGL \\
\hline BGO $33 \mathrm{C}$ & T12DCE & $1994 Q 1$ & u & $1 \mathrm{UGL}$ \\
\hline BGO 33C & T12DCE & $1994 Q 2$ & U & 1 UGL \\
\hline BGO 33C & T120CE & $1994 Q 3$ & U & $5 \mathrm{UGL}$ \\
\hline BGO $33 \mathrm{C}$ & T120CE & $1994 Q 4$ & U & 1 UGL \\
\hline BGO $33 \mathrm{C}$ & T12DCE & $1994 Q 4$ & $U$ & 1 UGL \\
\hline $\mathrm{BGO} 33 \mathrm{C}$ & T12DCE & $1994 Q 4$ & $u$ & $5 \mathrm{UGL}$ \\
\hline $\mathrm{BGO} 33 \mathrm{C}$ & T12DCE & $1994 Q 4$ & $u$ & 5 UGL \\
\hline BGO $33 \mathrm{C}$ & T12DCE & 199501 & $u$ & 1.67 UGL \\
\hline$B G 033 C$ & T12DCE & $1995 Q 4$ & $u$ & 2 UGL \\
\hline BGO $33 \mathrm{C}$ & T12DCE & $1996 Q 4$ & $u$ & 2 UGL \\
\hline BGO $33 \mathrm{C}$ & T12DCE & 199704 & $\mathrm{~J}$ & 0.364 UGL \\
\hline $\mathrm{BGO} 33 \mathrm{C}$ & T12DCE & 1998Q2 & UJ & $1 \mathrm{UGL}$ \\
\hline $\mathrm{BGO} 33 \mathrm{C}$ & T12DCE & 1998Q4 & JU & 5 UGL \\
\hline BGO $33 \mathrm{C}$ & T12DCE & $1998 Q 4$ & $U$ & 1 UGL \\
\hline BGO $33 \mathrm{C}$ & T12DCE & 1999Q3 & $u$ & 1 UC \\
\hline
\end{tabular}

traus-1.2-Dichloroethylene in $\mathrm{BGO} 33 \mathrm{C}$

$10-i$

152

*

$\underset{3}{3} 114$

总

76

38

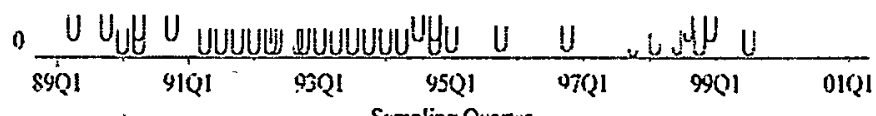

(Plor symbol: Res(pual if exists or in) 
$1,1,2,2-$ Tetrachloroethane in BGO $33 \mathrm{C}$

Data Retrieved by AWSA v1 from GIMS on 09NOV99 at 15:50

\begin{tabular}{|c|c|c|c|c|}
\hline Well & Analyte & Sample_Q & Res_Qual & Result \\
\hline BGO 33C & TCLEA & $1989 Q 2$ & $U$ & 5 UGL \\
\hline BGO $33 \mathrm{C}$ & TCLEA & 198903 & U & 5 UGL \\
\hline $\mathrm{BGO} 33 \mathrm{C}$ & TCLEA & $1989 Q 4$ & U & 5 UC \\
\hline BGO $33 \mathrm{C}$ & TCLEA & .199001 & $u$ & 1 UC \\
\hline $\mathrm{BGO} 33 \mathrm{C}$ & TCLEA & $1990 Q 2$ & $U$ & 1 UC \\
\hline BGO 33C & TCLEA & 199002 & $U$ & 1 UE \\
\hline BGO 33C & TCLEA & $1990 Q 2$ & $U$ & 5 UGL \\
\hline BGO 33C & TCLEA & 199002 & U & 5 UG \\
\hline BGO 33C & TCLEA & $1990 Q 3$ & $U$ & $5 U$ \\
\hline BGO 33C & TCLEA & $1990 Q 4$ & $U$ & $5 U$ \\
\hline $\mathrm{BGO} 33 \mathrm{C}$ & TCLEA & 1991Q1 & $u$ & 5 UGL \\
\hline BGO 33C & TCLEA & 1991Q2 & U & 1 UC \\
\hline $8 G O 33 C$ & TCLEA & 1991Q3 & $\mathbf{U}$ & $1 \mathrm{UE}$ \\
\hline $8 G 033 \mathrm{C}$ & TCLEA & $1991 Q 4$ & $\mathbf{u}$ & 1 UC \\
\hline$B G O 33 C$ & TCLEA & 1992Q1 & U & \\
\hline BGO 33C & TCLEA & 199202 & $U$ & \\
\hline BGO 33C & TCLEA & 1992Q3 & UJ & 1 UGL \\
\hline BGO 33C & TCLEA & $1992 Q 4$ & $\mathbf{U}$ & \\
\hline BGO $33 \mathrm{C}$ & TCLEA & 1993Q1 & $\mathbf{u}$ & $1 \cup G$ \\
\hline BGO 33C & TCLEA & 1993Q2 & $u$ & 1 UC \\
\hline BGO 33C & TCLEA & 1993Q3 & $u$ & 1 UC \\
\hline BGO 33C & TCLEA & $1993 Q 4$ & $U$ & 1 UC \\
\hline BGO 33C & TCLEA & 1994Q1 & U & 1 UGL \\
\hline BGO 33C & TCLEA & 199402 & $u$ & 10 \\
\hline BGO 33C & TCLEA & 1994Q3 & $\mathrm{U}$ & 5 UG \\
\hline BGO 33C & TCLEA & 1994Q4 & $\mathrm{U}$ & $1 \mathrm{UC}$ \\
\hline BGO 33C & TCLEA & $1994 Q 4$ & U & $1 U$ \\
\hline BGO 33C & TCLEA & 1994Q4 & $U$ & 5 uc \\
\hline BGO 33C & TCLEA & $1994 Q 4$ & $U$ & 5 UC \\
\hline BGO 33C & TCLEA & 1995Q1 & $u$ & 1.67 UG \\
\hline$B G O 33 C$ & TCLEA & $1995 Q 4$ & $u$ & 2 UG \\
\hline BGO 33C & TCLEA & 1996Q4 & $U$ & \\
\hline BGO 33C & TCLEA & $1997 Q 4$ & $U$ & \\
\hline BGO $33 \mathrm{C}$ & TCLEA & $1998 Q 2$ & UJ & 1 UG \\
\hline GO $33 C$ & TCLEA & $1998 Q 4$ & JU & \\
\hline 60330 & TCLEA & 1998Q4 & $\mathbf{U}$ & \\
\hline & & & $U$ & \\
\hline
\end{tabular}

$1,1,2.2-$ Teurachlornethane in $\mathrm{BGO} .3 \mathrm{C}$

5- Uี่บ แแU⿺

【li!

ul

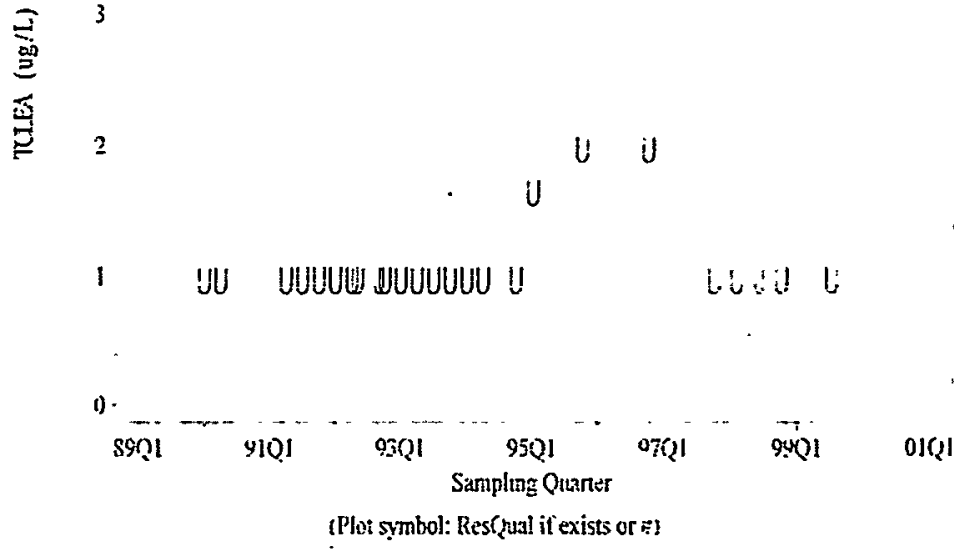


Zinc, total recoverable in BGO $33 \mathrm{C}$

Data Retrieved by AWSA v1 from GIMS on 23NOV99 at 08:45

Well Analyte Sample_Q Res_Qual Result Units BGO 33C ZNTOT 1996Q4 J 9.41 UGL BGO 33C ZNTOT 1997Q4 U BGO 33C ZNTOT $199802 \quad 32.4$ UGL BGO 33C ZNTOT 1998Q4 6.2 UGL BGO 33C ZNTOT $199804 \mathrm{~J} \quad$ - 5.5 UGL BGO 33C ZNTOT 1999Q3 11.9 UGL

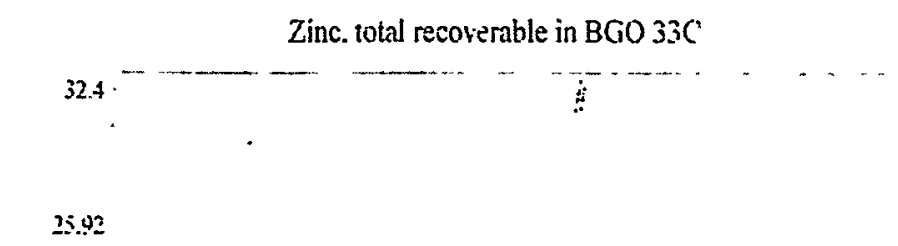

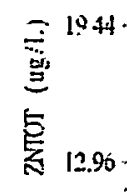

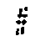

$d$

6.45

l: $\quad$ y

0 .

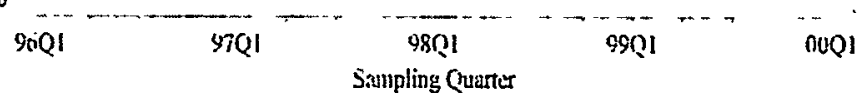

(Iln: șmbol. Kes(u) if exists or ä) 
Bromide in BGO $33 \mathrm{C}$

Data Retrieved by AWSA v1 from GIMS on 09NOV99 at 15:50

Well Analyte Sample_QRes_Qual Result Units

BGO 33C BR $1997 Q 4 \mathrm{~J}$

BGO 33C BR $199802 U$ U 50 UGL

BGO 33C BR $199802 \quad U \quad 65$ UGL

BGO 33C BR $1998 Q 4 \quad U \quad 250$ UGL

BGO 33C BR $1998 Q 4 U$ U

BGO 33C BR $199824 U$ U 250 UGL

BGO 33C BR $1998 Q 4 U$ U

BGO 33C BR 1999Q3 J

BGO 33C BR
49 UGL

87 UGL
Bromide in $\mathrm{BGO} 33 \mathrm{C}$

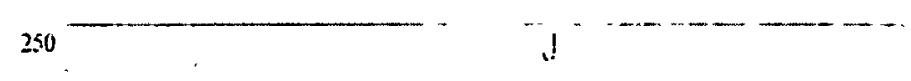

200

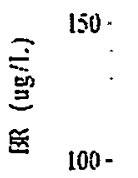

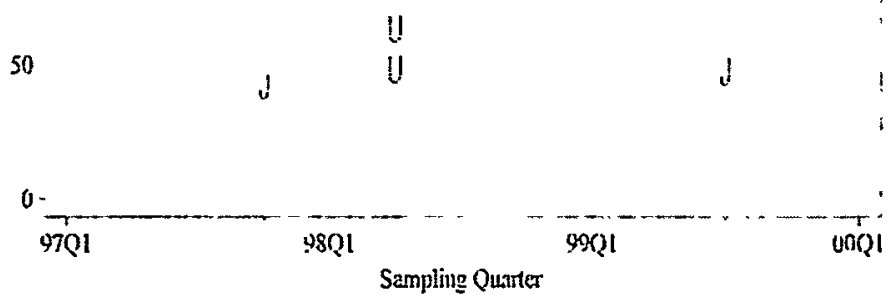

(Plot symhol: Res(lual ilevists or $\left.{ }^{\circ}\right)$ 